\title{
Overview of Environmental and Hydrogeologic Conditions at Galena, Alaska
}

By Allan S. Nakanishi and Joseph M. Dorava

U.S. GEOLOGICAL SURVEY

Open-File Report 94-525

Prepared in cooperation with the

FEDERAL AVIATION ADMINISTRATION

Anchorage, Alaska 


\section{U.S. DEPARTMENT OF THE INTERIOR}

BRUCE BABBITT, Secretary

U.S. GEOLOGICAL SURVEY

Gordon P. Eaton, Director

For additional information write to:

District Chief

U.S. Geological Survey

4230 University Drive, Suite 201

Anchorage, AK 99508-4664
Copies of this report may be purchased from:

U.S. Geological Survey

Earth Science Information Center

Open-File Reports Section

Box 25286, MS 517

Federal Center

Denver, CO 80225-0425 


\section{CONTENTS}

Abstract $\ldots \ldots \ldots \ldots \ldots \ldots \ldots \ldots \ldots \ldots \ldots \ldots \ldots \ldots \ldots \ldots \ldots \ldots \ldots \ldots \ldots \ldots$

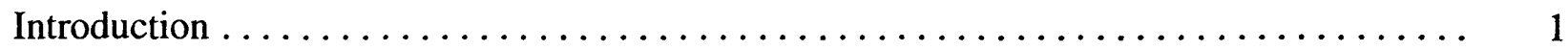

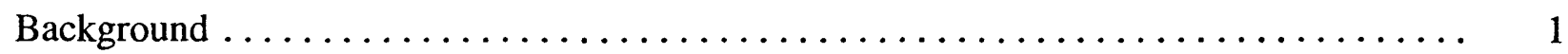

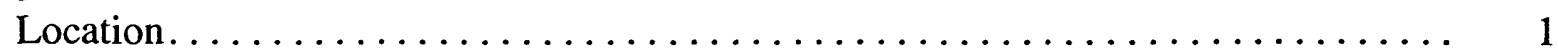

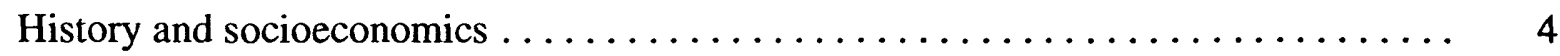

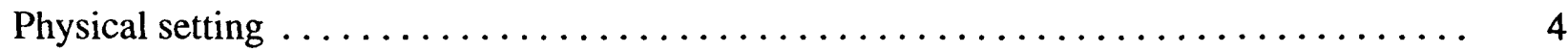

Climate $\ldots \ldots \ldots \ldots \ldots \ldots \ldots \ldots \ldots \ldots \ldots \ldots \ldots \ldots \ldots \ldots \ldots \ldots \ldots$

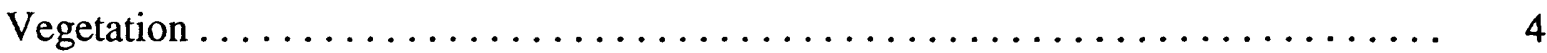

Bedrock geology ............................... 6

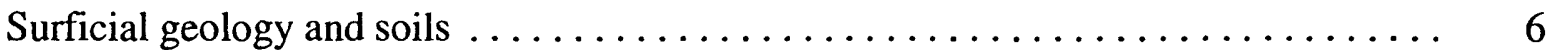

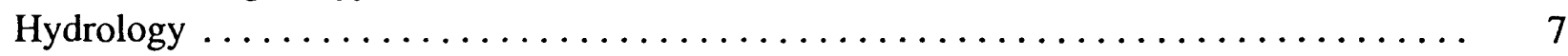

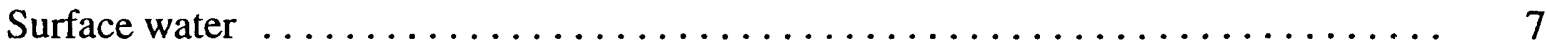

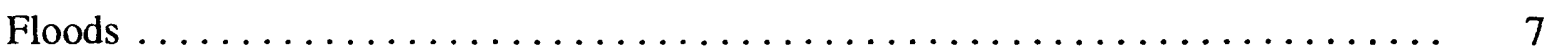

Flood-protection measures $\ldots \ldots \ldots \ldots \ldots \ldots \ldots \ldots \ldots \ldots \ldots \ldots \ldots \ldots \ldots$

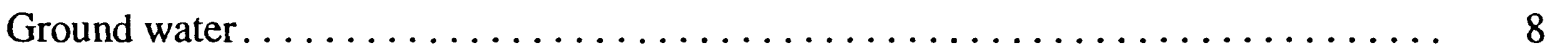

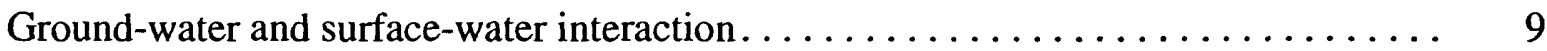

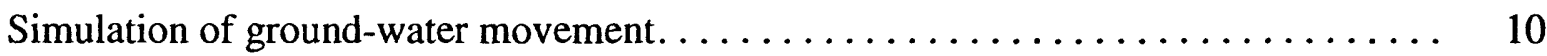

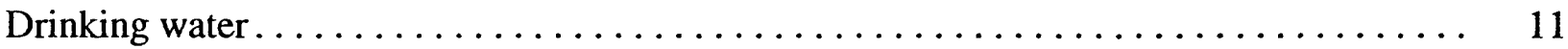

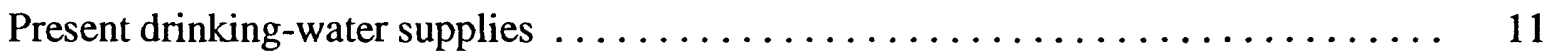

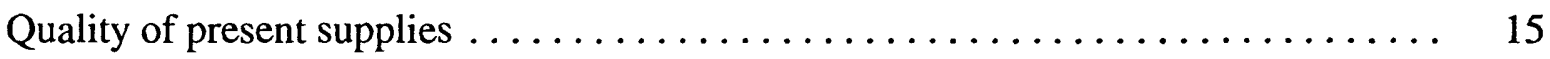

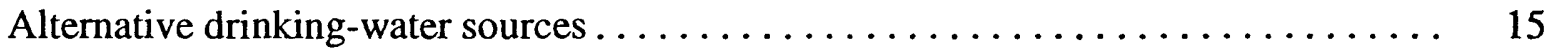

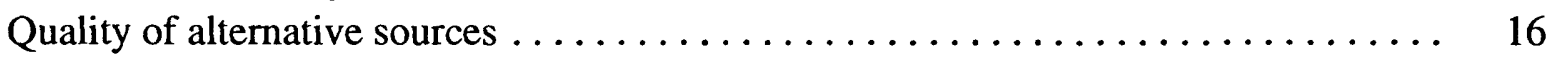

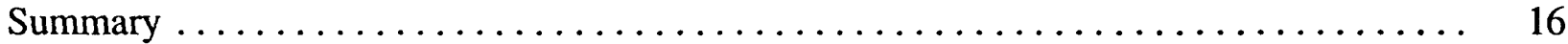

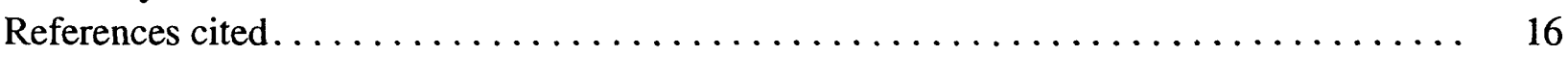

Appendix 1. Data, assumptions, justifications, and data sources used in the MODFLOW packages ............................ A-1

Appendix 2. Example output file of the U.S. Geological Survey Modular FiniteDifference Ground-Water Model . . . . . . . . . . . . . . . . A-2

Appendix 3. U.S. Army Corps of Engineers "Report on rehabilitation of well no. 1 water supply well, Galena AP" ................... A

Appendix 4. U.S. Public Health Service well drillers' logs, aquifer test data, and ground-water quality for Galena $\ldots \ldots \ldots \ldots \ldots \ldots \ldots \ldots \ldots \ldots \ldots$ A-4

Appendix 5. Summary of USGS Ground Water Site Inventory data, well drillers' logs, and water quality data for Galena $\ldots \ldots \ldots \ldots \ldots \ldots \ldots \ldots \ldots$ A-5

Appendix 6. U.S. Army Corps of Engineers "Report on Galena airport observation

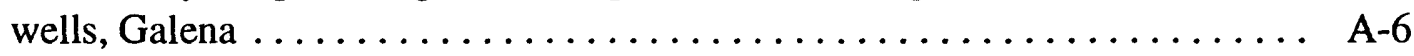

Appendix 7. U.S. Geological Survey water quality data for the Yukon River at Ruby and Yukon River at Galena . . . . . . . . . . . . . . . . . . . . . A-7 


\section{FIGURES}

1. Map showing location of Galena $\ldots \ldots \ldots \ldots \ldots \ldots \ldots \ldots \ldots \ldots \ldots \ldots \ldots \ldots \ldots$

2. Map showing location of Federal Aviation Administration facilities and Galena Air Force Station . . . . . . . . . . . . . . . . . . . . . .

3. Hydrograph of mean daily discharge for the Yukon River at Ruby for water years 1966-76.

4-6. Maps showing simulated water table contours and estimated flow direction of:

4. Shallow ground water with continuous permafrost effects in the

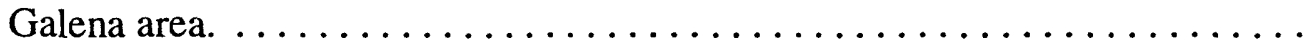

5. Shallow ground water with no permafrost effects and low streambed

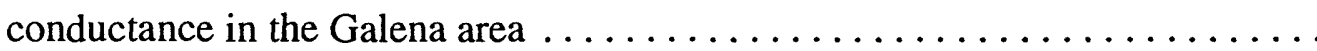

6. Shallow ground water with no permafrost effects and high streambed conductance in the Galena area ..........................

\section{TABLES}

1. Mean monthly temperature, precipitation and snowfall for the combined periods, 1922-76 and 1985-87, Galena . ........................... 5

2. Estimated peak discharges of the Yukon River for various recurrence intervals... 8

CONVERSION FACTORS, VERTICAL DATUM, AND ABBREVIATED WATER-QUALITY UNITS

\begin{tabular}{rll}
\hline Multiply & \multicolumn{1}{c}{ By } & To obtain \\
\hline millimeter $(\mathrm{mm})$ & 0.03937 & inch \\
centimeter $(\mathrm{cm})$ & 0.3937 & inch \\
meter $(\mathrm{m})$ & 3.281 & foot \\
kilometer $(\mathrm{km})$ & 0.6214 & mile \\
square kilometer $\left(\mathrm{km}^{2}\right)$ & 0.3861 & square mile \\
$\operatorname{liter}(\mathrm{L})$ & 0.2642 & gallon \\
cubic meter per second $\left(\mathrm{m}^{3} / \mathrm{s}\right)$ & 35.31 & cubic foot per second \\
liter per day $(\mathrm{L} / \mathrm{d})$ & 0.2642 & gallon per day \\
degree Celsius $\left({ }^{\circ} \mathrm{C}\right)$ & ${ }^{\circ} \mathrm{F}=1.8 \times{ }^{\circ} \mathrm{C}+32$ & degree Fahrenheit $\left({ }^{\circ} \mathrm{F}\right)$ \\
\hline
\end{tabular}

\section{Sea level:}

In this report "sea level" refers to the National Geodetic Vertical Datum of 1929--a geodetic datum derived from a general adjustment of the first-order level nets of both the United States and Canada, formerly called Sea Level Datum of 1929.

\section{Abbreviated water-quality unit used in this report:}

$\mathrm{mg} / \mathrm{L}$, milligram per liter 


\title{
Overview of Environmental and Hydrogeologic Conditions at Galena, Alaska
}

\author{
By Allan S. Nakanishi and Joseph M. Dorava
}

\begin{abstract}
The remote Native village of Galena along the Yukon River in west-central Alaska has long cold winters and short summers that affect the hydrology of the area. The Federal Aviation Administration owns or operates airway support facilities in Galena and wishes to consider the subsistence lifestyle of the residents and the quality of the current environment when evaluating options for remediation of environmental contamination at these facilities. Galena is located on the flood plain of the Yukon River and obtains its drinking water from a shallow aquifer located in the thick alluvium underlying the village. Surface spills and disposal of hazardous materials combined with annual flooding of the Yukon River may affect the quality of the ground water. Alternative drinking-water sources are available but at significantly greater cost than existing supplies.
\end{abstract}

\section{INTRODUCTION}

The Federal Aviation Administration (FAA) owns and (or) operates airway support, and navigational facilities throughout Alaska. At many of these sites, fuels and potentially hazardous materials such as solvents, polychlorinated biphenyls, and pesticides may have been used and (or) disposed of. To determine if environmentally hazardous materials have been spilled or disposed of at the sites, the FAA is conducting environmental studies mandated under the Comprehensive Environmental Response, Compensation, and Liability Act (CERCLA or "Superfund Act") and the Resource Conservation and Recovery Act (RCRA). To complete these environmental studies, the FAA requires information on the hydrology and geology of areas surrounding the sites. This report is the product of a compilation, review, and summary of existing hydrologic and geologic data by the U.S. Geological Survey, in cooperation with the FAA, for the FAA facilities and nearby areas at Galena, Alaska.

\section{BACKGROUND}

\section{Location}

Galena (fig. 1) is in the western interior of Alaska at latitude $64^{\circ} 44^{\prime} 10^{\prime \prime}$ N., longitude $156^{\circ} 56^{\prime} 04^{\prime \prime}$ W., approximately $910 \mathrm{~km}$ west of Fairbanks. The village is within the Koyukuk Flats (Wahrhaftig, 1965) on the north bank of the Yukon River, approximately $35 \mathrm{~km}$ upstream from the mouth of the Koyukuk River. It is the largest community of this region and serves as the transportation, government, and commercial center for the western Interior (Fison and Associates, 1987). A flood containment dike located immediately north of the old village of Galena, surrounds a 2,300-m runway, the Galena Air Force Station (AFS), and many of the FAA facilities (fig. 2). 


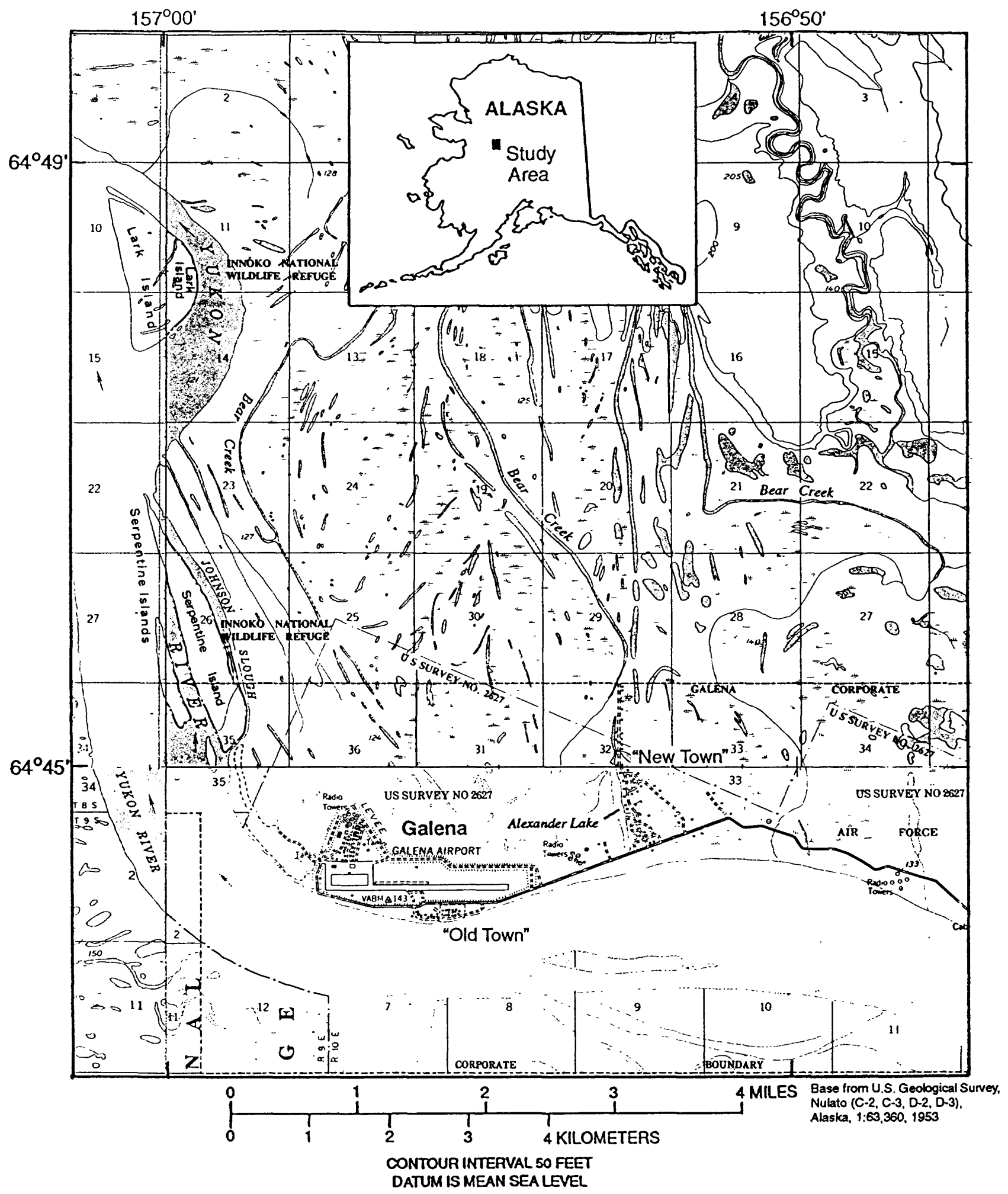

Figure 1. Location of Galena. 

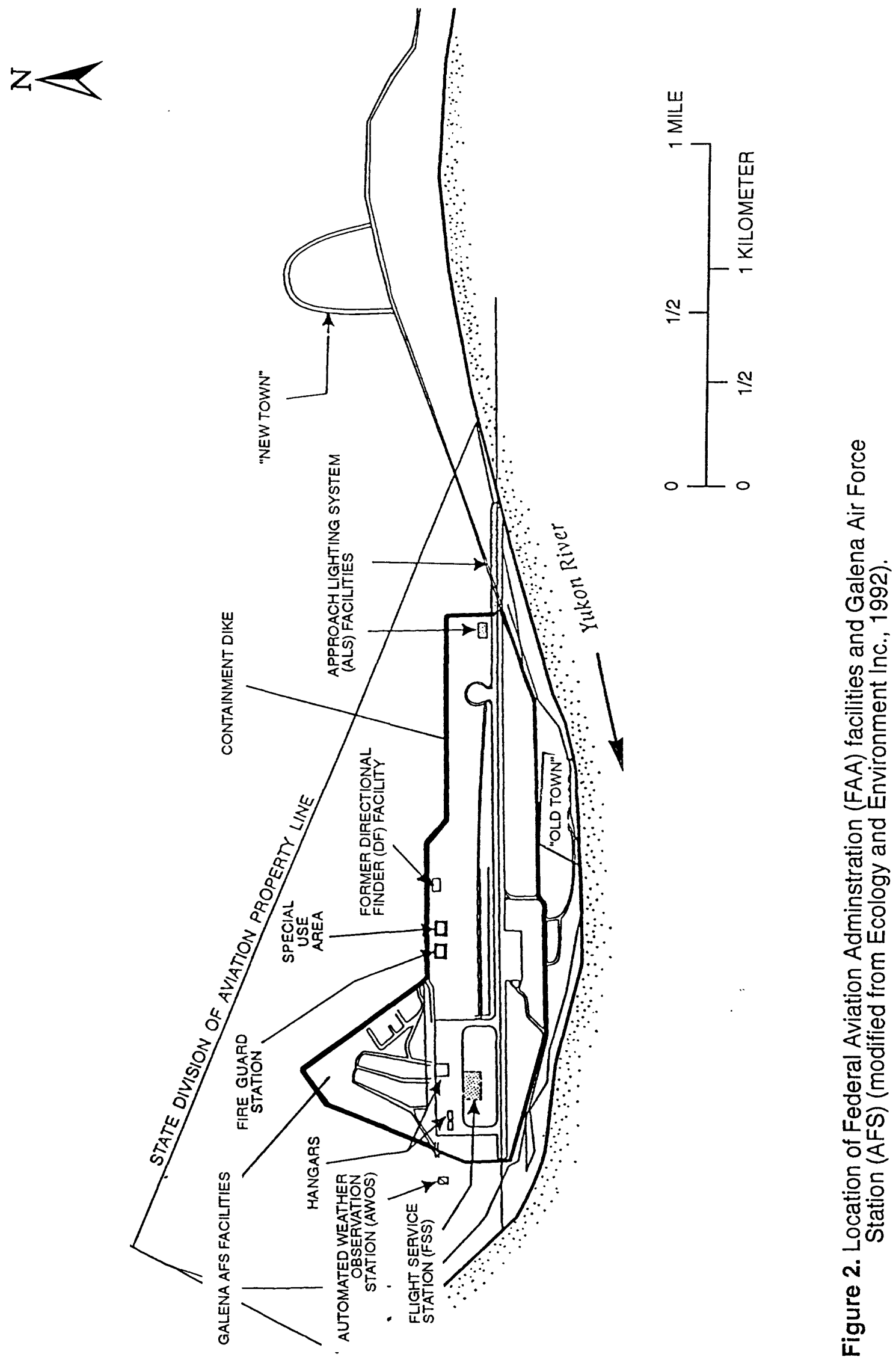


\section{History and Socioeconomics}

Galena is in traditional Koyukon Athabaskan Indian territory. The Koyukon frequently occupied large semi-permanent villages during the summers--most commonly near the primary fishing grounds--and hunted and trapped in the fall and winter along the rivers. Galena, located near an old fish camp site, was established in 1918 as a supply and trans-shipment point for the area lead ore mines. Significant community growth did not begin until the early 1950's, when the military established Galena AFS and Campion AFS, located $23 \mathrm{~km}$ southeast of Galena (Fison and Associates, 1987).

Galena is divided into two townsites (fig. 1). The original site "Old Town" is located immediately south of the airport. The other townsite, near Alexander Lake, is commonly referred to as "New Town" and was established in 1971, after flooding severely damaged the original townsite. Galena was incorporated as a first-class city in 1971. The municipal government is directed by a city manager who administers local programs.

In October 1993, the U.S. Air Force completed the drawdown (closing) of the Galena AFS. However, the facilities are being maintained by contractors to minimize deterioration should the Air Force decide to reopen the station. This drawdown had a significant economic effect on the village of Galena. Approximately 325 Air Force personnel were relocated, resulting in a population decline of more than one-third from the 1990 population of 829 (Alaska Department of Community and Regional Affairs, 1993). The percentage of Native population, which includes American Indian, Eskimo, and Aleut, has increased from 45 percent to an estimated 70 percent as a result of the station closure (Chris Hladick, Galena City Manager, oral commun., 1994). Federal, State, and village government jobs dominate the economy, but Galena has many other jobs in air transportation and retail businesses. Strong cultural, historic, and family ties to subsistence activities make subsistence foods a major portion of the diet for many Galena residents.

\section{PHYSICAL SETTING}

\section{Climate}

The climate of Galena is continental which characteristically has low precipitation, low cloudiness, low humidity, light surface winds, and large annual temperature variations (Hartman and Johnson, 1984). Freezing of the Yukon River typically occurs in October and break-up occurs in mid-May (Fountain, 1984; Fountain and Vaughn, 1984). The mean annual "temperature is $-4.4^{\circ} \mathrm{C}$, but temperatures range from a July mean maximum of $19.7^{\circ} \mathrm{C}$ to a January mean minimum of about $-27.6^{\circ} \mathrm{C}$. Mean annual precipitation is about $327 \mathrm{~mm}$; approximately $1,537 \mathrm{~mm}$ of snow falls annually (Leslie, 1989). Most rainfall occurs in July and August. Mean monthly temperature, precipitation, and snowfall are summarized in table 1.

\section{Vegetation}

The Galena area forest consists of a closed spruce-hardwood forest along the Yukon River (Viereck and Little, 1972). Well-drained, high relief areas contain aspen, birch and white spruce (Elias and Vosburgh, 1946; Weber and Péwé, 1970). The flat, poorly drained terraces consist predominantly of black spruce, larch, and moss undergrowth. Poorly drained, flat-lying sloughs and sediment-filled lakes contain moss, lichen, tufted grass, alder brush, Labrador tea and scattered willows (Elias and Vosburgh, 1946; Péwé, 1948; Weber and Péwé, 1970). 


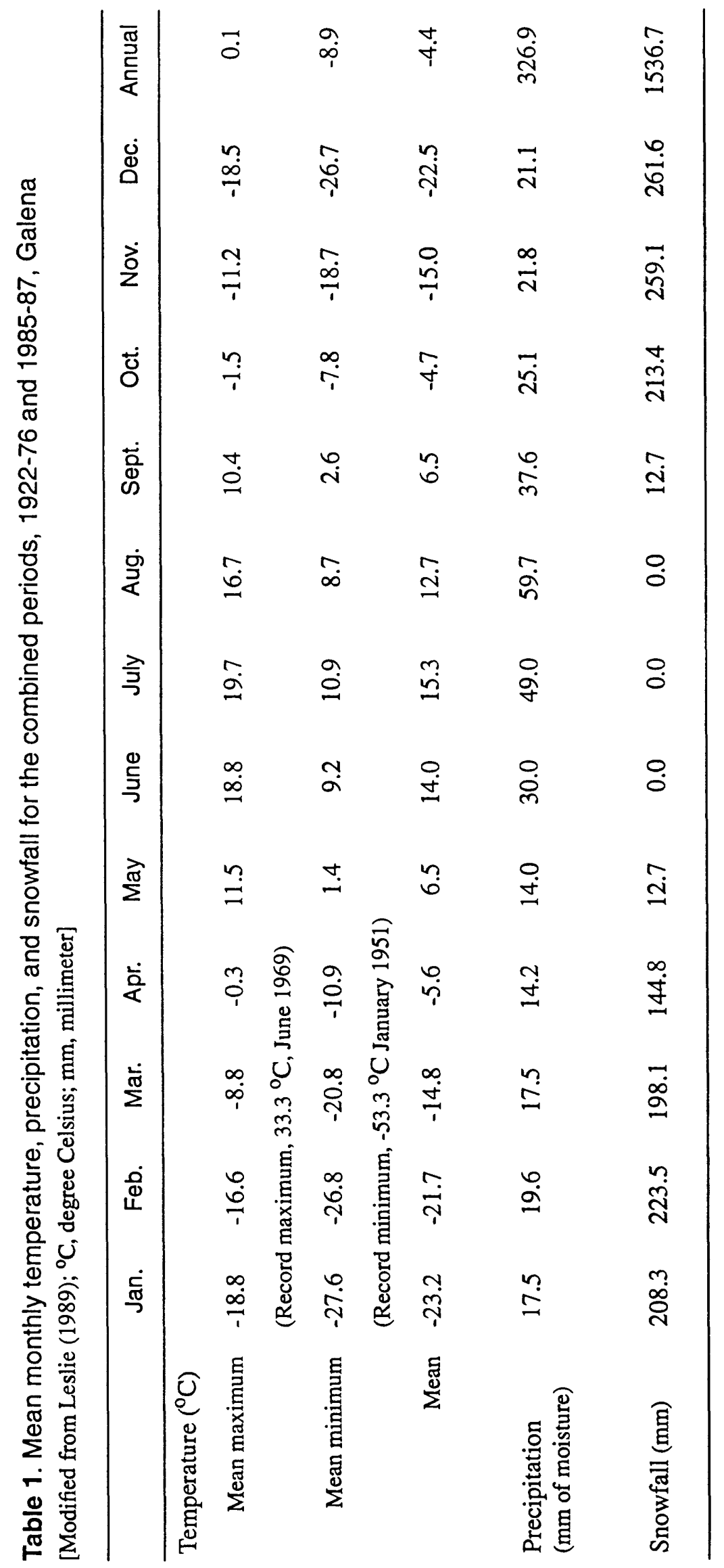




\section{Bedrock Geology}

The broad flats surrounding Galena are bounded by hills and mountains to the south, northwest and northeast ranging from $300 \mathrm{~m}$ to more than $600 \mathrm{~m}$ in elevation. Mueller Mountain, Bishop Rock, and Pilot Mountain -- located $25 \mathrm{~km}$ east, $25 \mathrm{~km}$ northwest, and $20 \mathrm{~km}$ west of Galena, respectively -- are the closest bedrock exposures to Galena. These outcrops have been studied for use as potential construction sites and as sources for construction material (Elias and Vosburgh, 1946; U.S. Army Corps of Engineers, 1986). Bedrock exposures consist primarily of sedimentary, igneous, and volcanic rocks. The sedimentary rock consists of Cretaceous age sandstone, shale, and conglomerate. Volcanic rock of undetermined age is found in isolated exposures and consists of basaltic to andesitic rock (Cass, 1959). Exposed metamorphic rocks of Paleozoic age consist of schist, phyllite, slate and quartzite (Cass, 1959). The depth to bedrock at Galena is unknown. A well at Campion AFS was drilled to $130 \mathrm{~m}$ without reaching bedrock.

\section{Surficial Geology and Soils}

Rieger and others (1979) classified soils in the State of Alaska, which included the Yukon Flats area, using the Soil Classification System developed by the U.S. Soil Conservation Service. Weber and Péwé (1970) characterized the surficial deposits and engineering properties of the soils for the Koyukuk Flats.

Thick deposits of Quaternary sediment overlie most of the bedrock in the Galena area (Cass, 1959). These deposits consist of flood-plain alluvium and river terrace deposits. Flood plain alluvium is divided into organic-rich and organic-poor deposits. Organic-rich alluvial deposits consist of well-stratified layers and lenses of silt with wood, peat, and other intermixed organic materials. Organic-rich deposits occupy the upper layers of the flood-plain alluvium and are formed in abandoned channels and cutbanks of major streams. Deposit thickness ranges from 2 to $20 \mathrm{~m}$ and depth to permafrost ranges from 0.3 to $0.7 \mathrm{~m}$.

Organic-poor alluvial deposits consist of well-stratified layers and lenses of silt with some sand, gravel, and minor quantities of clay and slightly organic-rich silt. These deposits occupy the lower layers of flood-plain alluvium and are formed in the borders of major streams and in abandoned channels. Deposit thickness ranges from 1 to $20 \mathrm{~m}$. Permafrost is probably absent or at depths of more than $6 \mathrm{~m}$ near rivers, but at interlake areas, permafrost depth can be as little as 0.3 to $1 \mathrm{~m}$ and possibly more than $3 \mathrm{~m}$ under the lake basins.

Terrace deposits consist of well-stratified layers and lenses of silt, organic-rich silt, and localized deposits of well-stratified fine to coarse-grained sand. Terrace deposits are found bordering the Yukon and Koyukuk River flood plains as remnant ridges on low flat areas 10 to $60 \mathrm{~m}$ above the river level or as outer margins of the lowland bordering the hills and extending up some of the smaller stream valleys. The total deposit thickness may be more than $150 \mathrm{~m}$ and the depth to permafrost is about $0.3 \mathrm{~m}$.

The local area is underlain by a layer of discontinuous permafrost (Ferrians, 1965). Seasonal frost commonly penetrates to a depth of about $2 \mathrm{~m}$; however, ground temperatures measured near the airport indicate that soil temperatures at a depth below about $2 \mathrm{~m}$ were consistently above freezing continuously for more than 11 years (Aitken, 1963). Significant melting of permafrost has occurred since the construction of the Galena AFS. A geotechnical study by Péwé (1948) showed 
that the depth to permafrost was about 2 to $3 \mathrm{~m}$ and was continuously present at all locations except in areas nearest to the Yukon River. Recent geotechnical investigations at the airport facilities have found isolated lenses of permafrost or no permafrost during a monitoring well-drilling program (Wes Lannen, U.S. Air Force, 11th CEOS, Installation Restoration Program, Project Manager, oral commun., 1994).

\section{HYDROLOGY}

\section{Surface Water}

The Koyukuk Flats is drained by the Koyukuk and the Yukon Rivers. The Yukon River is Alaska's largest river and the fifth largest river in North America in terms of drainage area and runoff (Feulner and others, 1971). The Yukon River flows roughly from east to west and drains into the Bering Sea about $310 \mathrm{~km}$ downstream from Galena. The Koyukuk River drains into the Yukon River about $35 \mathrm{~km}$ west of Galena. Numerous meander scars, sloughs, and oxbow lakes indicate a dynamic fluvial system within the Koyukuk Flats.

Surface-water bodies within a 3-km radius of the airport include the Yukon River, Alexander Lake, and the headwaters for Bear Creek (fig. 1). Galena is on the interior and upstream section of a meander bend on the north bank of the Yukon River and is subject to bank erosion. Riverbank erosion at an average rate of $4.7 \mathrm{~m} / \mathrm{yr}$ between 1946 to 1978 was measured by the U.S. Army Corps of Engineers (1986) and threatens the eventual destruction of the airstrip, military facilities, FAA facilities, and parts of the old village of Galena.

\section{Floods}

Historically, flooding near Galena occurs in mid-May during the break-up of the Yukon River. Flood hazards in Galena are considered high by the U.S. Army Corps of Engineers (1993); 95 houses and 1 public facility are within the 100 -year flood zone. The primary cause of flooding in the Galena area is ice jams.

Ice-jam flooding occurs when river ice broken during spring thawing is transported downstream and its downstream movement is blocked in locations where a constriction, a sandbar, or other obstruction such as a sharp meander bend exists (Beltaos, 1990). The blockage prevents ice movement and restricts water flow as the ice jam builds in thickness and length. This subsequently slows the water velocity and produces a rise in water level or backwater effect that propagates upstream from the ice jam. When the ice jam releases, a flood wave propagates downstream.

Significant floods have been reported since 1925, when records of flooding began. In 1945, ice-jam flooding destroyed most of the village. The flood of record is the 1971 ice-jam flood, during which water reached depths of $2.4 \mathrm{~m}$ at "Old Town" and $1.9 \mathrm{~m}$ at the Galena airport. Most of the town was destroyed by the flood and the new townsite was established $2.4 \mathrm{~km}$ to the east. Most of the population, however, still remains in the old townsite (Ecology and Environment Inc., 1992).

Flooding at Galena not only damages structure and roads, but also causes contaminants on the surface of the land to mobilize and move into inadequately sealed wells. Flood waters can reach depths that are higher than the top of well casings. Even for wells that are effectively sealed, flood water may move contaminants into previously uncontaminated areas, where they can then infiltrate into the aquifer. 
The flood frequency table for Ruby (table 2) was obtained using the graph of discharge to drainage area for the Yukon River developed by Jones and Fahl (1994, fig. 10). The drainage area for the Yukon River basin upstream from Galena is about $716,800 \mathrm{~km}^{2}$ (Federal Emergency Management Agency, 1983). The flood frequency curves developed by Jones and Fahl apply only to floods generated by rainfall and snowmelt runoff and are not applicable to ice-jam floods.

Table 2. Estimated peak discharges of the Yukon River at Ruby for various recurrence intervals

[Discharge is in cubic meters per second]

\begin{tabular}{ccccccc}
\hline \multicolumn{8}{c}{ Recurrence interval } \\
\hline 2 years & 5 years & 10 years & 25 years & 50 years & 100 years & 500 years \\
\hline 18,700 & 23,100 & 25,600 & 28,500 & 30,300 & 32,300 & 36,000 \\
\hline
\end{tabular}

\section{Flood Protection Measures}

The Air Force began construction of a flood containment dike (fig. 2) north of "Old Town" in 1944 after the runway and camp area were inundated by an ice-jam flood during the spring. An ice-jam flood in May 1945 overtopped the dike which was subsequently raised another $4.3 \mathrm{~m}$. The flood containment dike surrounding the Air Force Station was constructed at an elevation of $41.5 \mathrm{~m}$. The Federal Emergency Management Agency (1983) considers the flood containment dike as adequate protection from the 100-year flood.

The base flood elevation for ice-jam floods is about $40.8 \mathrm{~m}$ at "Old Town" and about $41.0 \mathrm{~m}$ in "New Town." Both townsites, however, are located outside of the flood containment dike (U.S. Army Corps of Engineers, 1983, 1986, 1993).

\section{Ground Water}

Ground-water recharge to the Galena area occurs from precipitation, infiltration, and groundwater movement from areas near the slopes of the surrounding highlands. Ground-water discharges into local surface-water streams and sloughs which drain into the Yukon River." Ground watermovement is influenced by impermeable lenses or layers of permafrost. The area-wide variability in the presence of permafrost accounts for the local occurrence of sub-, intra-, and suprapermafrost ground water (Woodward-Clyde Consultants, 1989). Previous studies on the subject of ground water and permafrost include reports by Cederstrom and others (1953), Hopkins and others (1955), and Williams and Waller (1963).

Alluvium is probably unfrozen beneath the bed of the Yukon River throughout its course in Alaska and is probably frozen beneath the flood plain adjacent to the river. Frozen alluvium is probably thickest beneath sections of the flood plain and low terraces that are away from present rivers and lakes. Most of the wells in the Yukon River villages from Canada to the Bering Sea are along the riverbank where the warming effect of the river affects the thickness of frozen ground (Smith, 1986). Water levels, where observed in these wells, fluctuate with the stage of the river. 
Frozen alluvium forms wedge-shaped masses that are thin near the river, but thicken up to $34 \mathrm{~m}$ in the northern part of the Galena AFS (Péwé, 1948; Woodward-Clyde Consultants, 1989). Suprapermafrost ground water was found at an average depth of $6 \mathrm{~m}$ in a site contamination study by Woodward-Clyde Consultants (1989). Subpermafrost ground water confined beneath the frozen ground rises to a static level between 6 to $12 \mathrm{~m}$ below the ground surface. Campion AFS, on a high terrace $23 \mathrm{~km}$ east of Galena, is supplied by wells drilled though frozen silt, clay, and sand to depths of 123 to $129 \mathrm{~m}$ where they reached unfrozen water-bearing gravel (Williams, 1970). Water confined beneath the frozen ground rose approximately $46 \mathrm{~m}$ above the base of the permafrost in these wells (Williams, 1970).

\section{Ground-Water and Surface-Water Interaction}

The variations in river stage and ground-water elevations at Galena will generally follow the pattern of the discharge hydrograph for the Yukon River at Ruby (fig. 3). Continuous streamflow records are not available for the Yukon River at Galena; however, the mean daily discharge record for the period 1956-78 is available for the Yukon River at Ruby located about $80 \mathrm{~km}$ upstream from Galena (U.S. Geological Survey, 1957-79). The stream-gaging station at Ruby has a record of discharge representing approximately $671,000 \mathrm{~km}^{2}$ of drainage area (Federal Emergency Management Agency, 1983). The Ruby gaging station represents about 94 percent of the Yukon River drainage basin upstream from Galena.

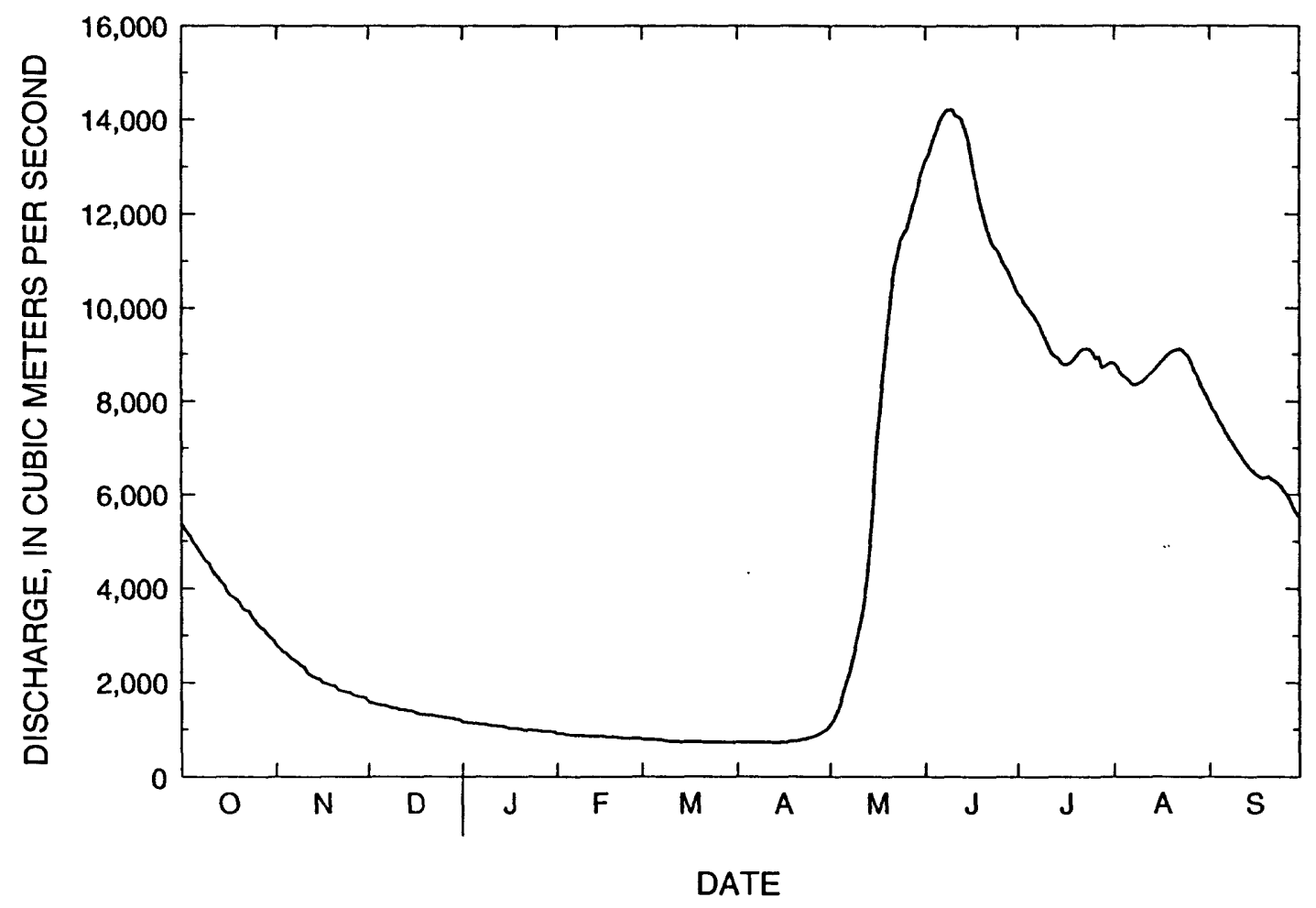

Figure 3. Mean daily discharge for the Yukon River at Ruby, water years 1966-76. 
Adjacent to the river, shallow ground-water flows into and out of the riverbanks depending on the elevation of water in the river relative to the water table. Seasonally, the discharge of the river will fluctuate from a maximum in late May or early June to a minimum in late April or early May (fig. 3). The river also rises during late-summer rainstorms. The water table generally rises and falls in response to these river fluctuations and is attenuated with distance from the river. This flow of water into and out of the aquifer in response to changing stage of the river is termed "bank storage effects" (Linsley and others, 1982). Bank storage effects have not been studied at Galena. Because the airport, FAA facilities and village utilities are adjacent to the river, bank storage effects could have a significant influence on the movement and extent of contamination.

\section{Simulation of Ground-Water Movement}

A mathematical ground-water model approximates the directions and rates of water movement through an aquifer system. Partial-differential equations thought to represent the physical processes of ground-water flow are solved by the model and require that the hydraulic properties and boundaries be defined for the modeled area. The aquifer system was overlain by a grid, which was extended in the third dimension to form blocks or "cells." The cells form rows, columns, and layers. Each cell in the model grid represents a block of permeable material within which the hydraulic properties are assumed to be uniform. Any specific cell may be referenced by citing its row, column, and layer location. The limits of the modeled area were selected to include or nearly coincide with natural flow boundaries. The "boundary surface" of the flow region corresponds to identifiable hydrogeologic features at which some characteristic of ground-water flow can be described. For the conceptual model, these features could be a drainage divide, a river bank, or artificially induced (depending on the modelled area). In cases where there is no apparent natural flow boundaries, such as in an open flood plain, the model grid was extended far enough away from the area of study so the error created from the artificial boundary is minimized.

Ground-water flow in the Galena area was simulated using a computer program, MODFLOW (McDonald and Harbaugh, 1988), as a simple steady-state conceptual model. Under steady-state conditions, the recharge to the system is equal to the discharge from the system, no water is derived from storage, and there is no change in head with time. Output from MODFLOW was graphically presented using METAZ, a contouring program specifically designed for MODFLOW and developed by S.A. Leake and R.T. Hanson (U.S. Geological Survey, written commun., 1993). The conceptual model requires that the hydraulic head at the aquifer boundaries is known, all recharge and discharge is assumed to occur at the river, flow is horizontal, and the aquifer materials are homogeneous and isotropic. The data, assumptions, justifications, and data sources used in the model packages are summarized in appendix 1. An example output file of the model is shown in appendix 2. The purpose of undergoing a mathematical ground-water simulation was to identify hydrologic features that may have a significant influence on the ground-water flow direction in the Galena area. Two ground-water flow simulations were used to identify features having the greatest influence on ground-water flow direction.

The westward-flowing Yukon River is an important factor in establishing the general westward direction of ground-water flow. The hydraulic gradient of the aquifer is strongly influenced by the surface-water gradient of the Yukon River which was measured by the U.S. Army Corps of Engineers (1983). The hydraulic continuity of the unconsolidated alluvium away from the river will have a profound influence on the directions of ground-water flow. If the permafrost in the area is discontinuous, the unconsolidated alluvium will behave like an aquifer. If it is continuous, the unconsolidated alluvium will act as a confining layer. 
The first simulation is based on the assumption that permafrost north of Galena is continuous and blocks northward ground-water flow. The thawed alluvium near the Yukon River was simulated as a narrow, strip aquifer running parallel to the Yukon River. The strip aquifer was estimated to be approximately $500 \mathrm{~m}$ in width along the north bank of the Yukon River. This aquifer width was extended to about $800 \mathrm{~m}$ at cleared sites in the vicinity of Galena to reflect the thawing conditions of vegetation-free areas. Under steady-state conditions and assuming the conditions stated above, the simulated ground-water flow direction at Galena is generally towards the west-southwest (fig. 4), parallel to the river.

A second simulation was based on the assumption that the permafrost near Galena is not a barrier to ground-water flow north of the Yukon River. In this simulation, Bear Creek (fig. 1), a northward flowing stream system located immediately north of Galena, gains water from the shallow aquifer and may have a significant influence on ground-water flow directions at Galena. In the absence of permafrost, the influence of the Bear Creek drainages on ground-water flow depends primarily on the vertical hydraulic conductance of its streambed. Higher streambed conductances permit greater quantities of water to flow between the aquifer and the stream.

Two model runs were performed simulating the Bear Creek drainage with low and high streambed conductances. The model simulation of low streambed conductance shows that ground water at Galena remains flowing generally in a southwestward direction, towards the Yukon River (fig. 5). The model simulation of high streambed conductance shows that ground water at Galena now flows in a westward to northwestward direction, towards the Bear Creek drainage (fig. 6). The simulations of low and high streambed conductance show that ground-water flow can be influenced, up to a 90 degree difference in direction, by altering the model input of the hydraulic properties of Bear Creek. Stream property information such as discharge, depth, stage, bed thickness, bed slope, and permafrost conditions are needed to refine the definition of the effects of the Bear Creek drainage on the ground-water flow in the Galena area. These shallow ground-water models, however, define the constraints in possible flow direction.

Three model simulations, each using different assumptions of the continuity of the permafrost and streambed conductance (figs. 4, 5, and 6), illustrate the importance of field investigations to identify the role of permafrost and the hydraulic connection between Bear Creek and the aquifer. Without field data, flow directions can not be ascertained exactly, but can only be described generally on the basis of assumed boundary conditions.

\section{DRINKING WATER}

\section{Present Drinking Water Supplies}

Residents of Galena obtain their drinking water from wells (U.S. Army Corps of Engineers, 1987), which are owned by the military, the municipal government, and other institutions. The Galena AFS obtained its water supplies from two 60-m deep wells constructed into the alluvium near the banks of the Yukon River. A U.S. Army Corps of Engineers report (appendix 3) describes an early contaminant rehabilitation study on one of the two primary wells used by the Air Force. The two wells provided a domestic water supply for approximately 300 personnel at the Air Force station. The water wells at the airport have been found to contain petroleum and chlorinated solvents (Woodward-Clyde Consultants, 1989). As a result of this contamination, two wells serving the air passenger terminals have been abandoned. 


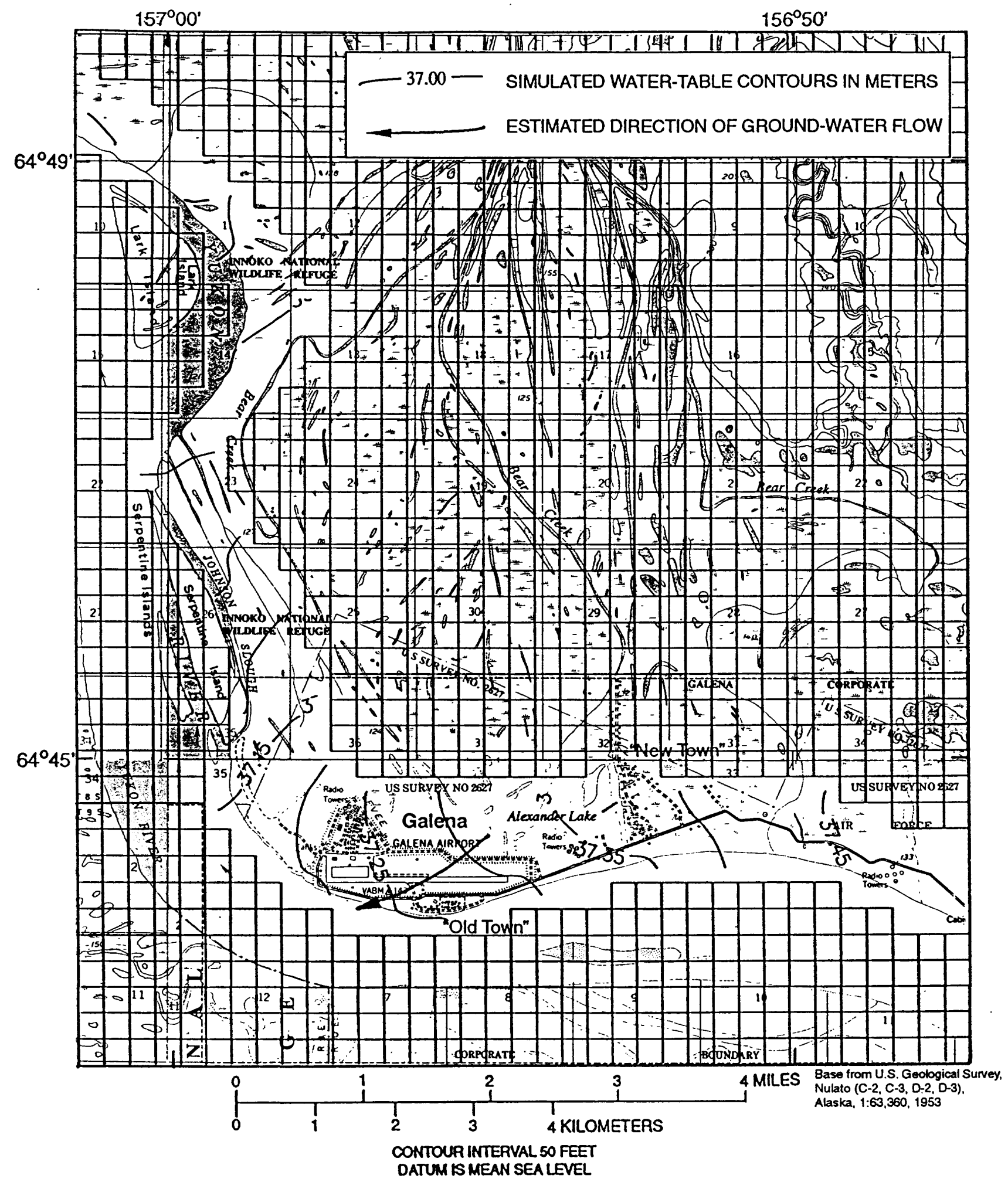

Figure 4. Simulated water-table contours and estimated flow direction of shallow ground water with continuous permafrost effects in the Galena area. 


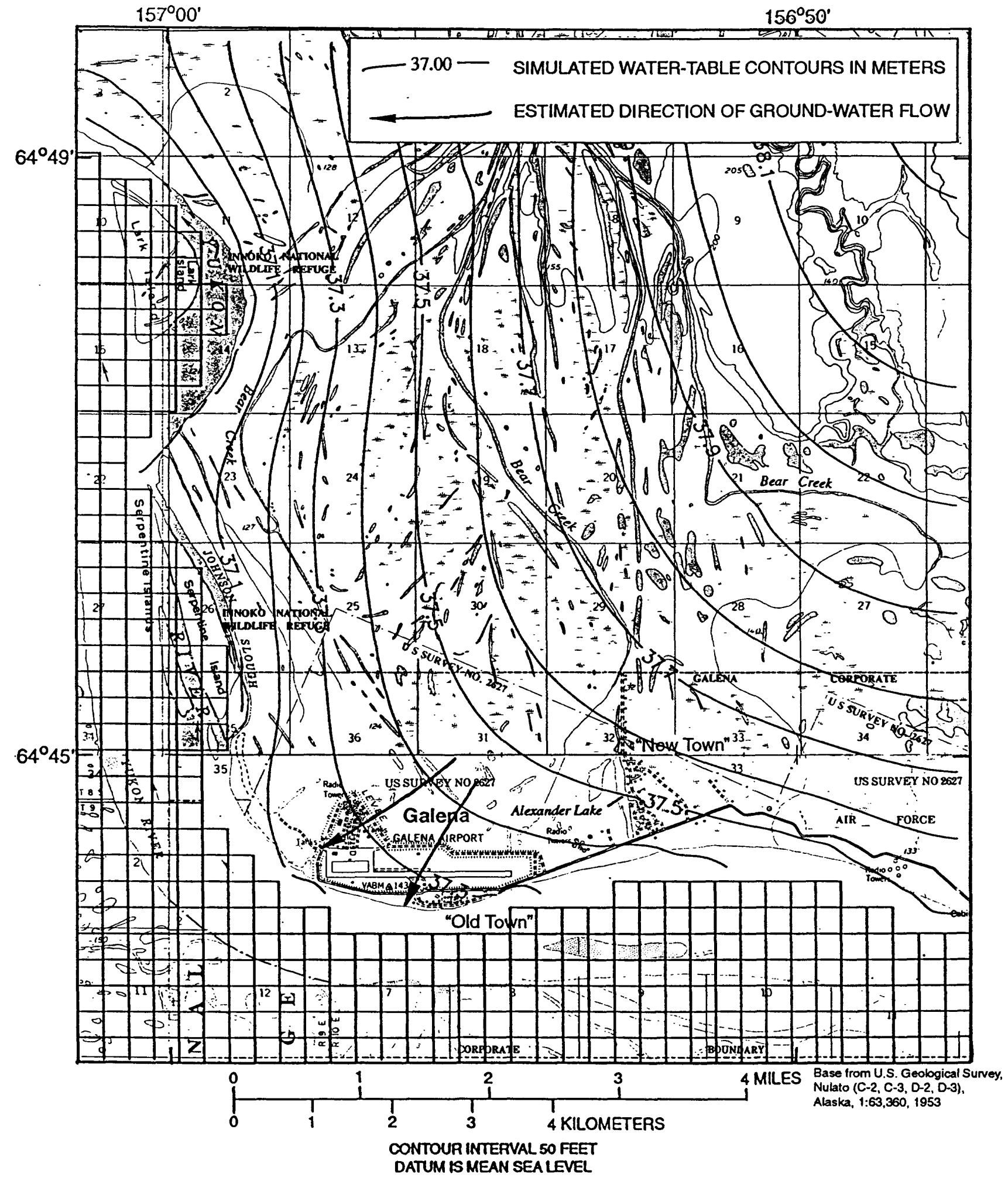

Figure 5. Simulated water-table contours and estimated flow direction of shallow ground water with no permafrost effects and low streambed conductance in the Galena area. 


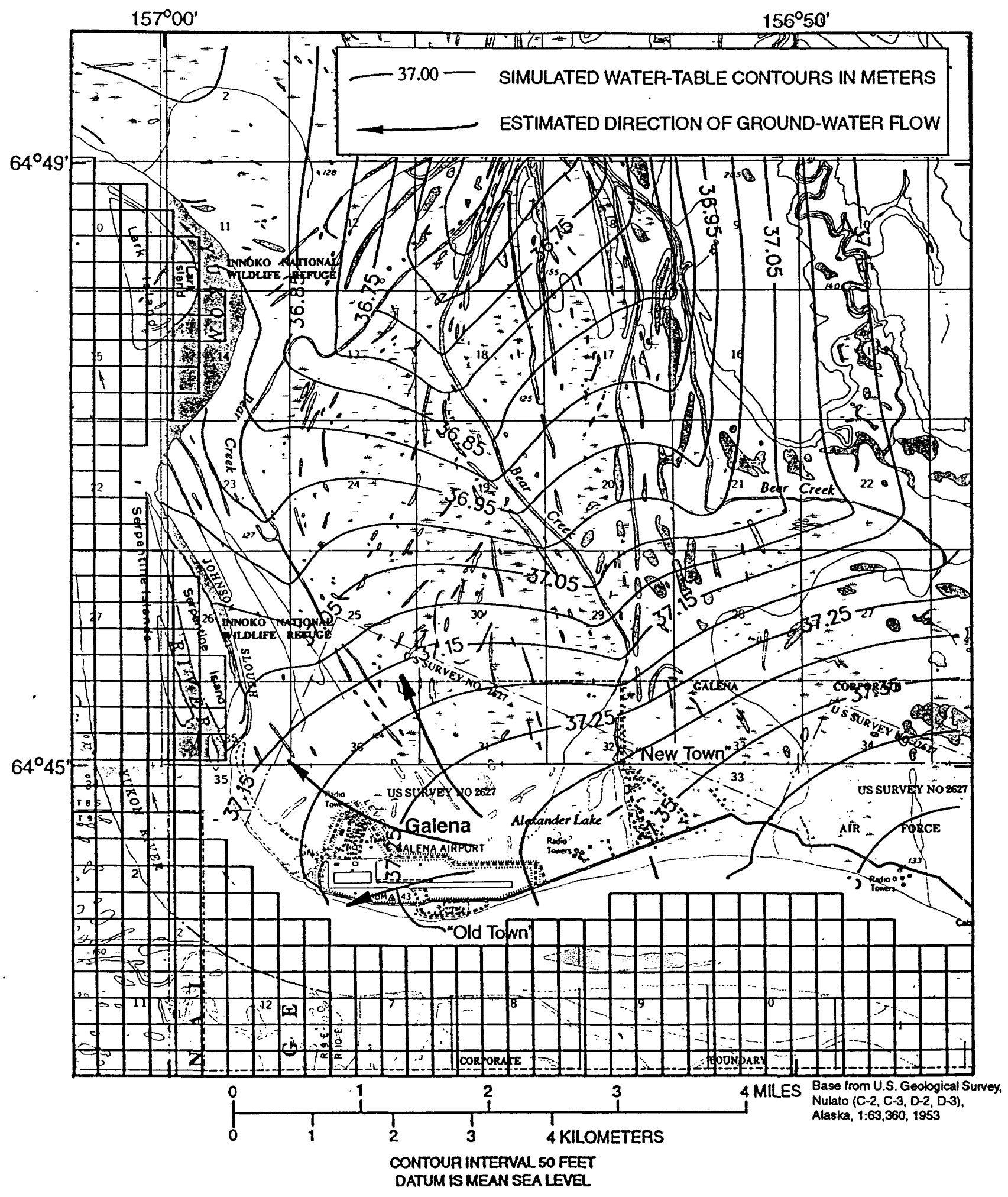

Figure 6. Simulated water-table contours and estimated flow direction of shallow ground water with no permafrost effects and high streambed conductance in the Galena area. 
The village of Galena operates its own municipal water system. Drinking water is obtained from a $47-\mathrm{m}$ drilled well with a $15.2-\mathrm{cm}$ diameter steel casing. The water is pumped from the well to the water-treatment facility and then stored in a $98,400-\mathrm{L}$ wood stave storage tank (Fison and Associates, 1987). Water is distributed to a central watering point (washeteria) and by a pipe system primarily to the newer homes in the area.

\section{Quality of Present Supplies}

Williams (1970) characterized the water quality of ground water in permafrost regions. Permafrost may have a strong influence on the water quality in the area. Water found above the permafrost (suprapermafrost water) is generally unpotable unless treated extensively. Usually found within 1 to $8 \mathrm{~m}$ of the surface, it commonly has a high mineral and (or) organic content and is susceptible to surface contamination. The quantity of water from this source is often low and unreliable. Water found below permafrost (subpermafrost water) is generally deficient in dissolved oxygen. As a result, high concentrations of some minerals are present, such as iron and manganese, which are soluble under these conditions. Subpermafrost ground water is commonly hard and occasionally contains dissolved organic substances (Williams, 1970).

Sporadic records on the water quality in the Galena area are available from the U.S. Public Health Service for the periods 1977-78 (appendix 4), from the USGS for the periods 1959-70 (appendix 5), and from the U.S. Army Corps of Engineers for 1963 (appendix 6). Analysis of untreated samples taken from various locations in Galena indicated an average silica content of $31 \mathrm{mg} / \mathrm{L}$, an average hardness as $\mathrm{CaCO}_{3}$ of $214 \mathrm{mg} / \mathrm{L}$, and an average iron content of $2.2 \mathrm{mg} / \mathrm{L}$. Silica and hardness may create scale in plumbing and boilers but are of little health concern to most users. The average iron content for Galena wells is higher than the $0.30 \mathrm{mg} / \mathrm{L}$ secondary maximum contaminant level regulations set by the USEPA (1993) for drinking water. A high iron content, however, does not prohibit this water from being used for drinking.

\section{Alternative Drinking Water Sources}

Information on alternative drinking-water sources in the Galena area is needed in order to plan actions that would be taken if the present drinking-water source became contaminated. Alternative sources might be a nearby surface-water source or aquifers that are separated from the present water-supply aquifer by a tight confining layer. The aquifer system at Galena has not been mapped in sufficient detail to define individual aquifers and confining layers. It is possible that multiple aquifers exist, separated by a permafrost confining layer. The areal continuity of the permafrost is uncertain, however, as is its role in inhibiting movement of ground water and possibly contaminants to the subpermafrost aquifer.

According to Elias and Vosburgh (1946), Alexander Lake (fig. 1), located near "New Town," could be a perennial supply of water. Other surface-water bodies in the Galena area, such as ponds and sloughs, may not be viable alternatives to ground water as a drinking-water source. If the water body is too shallow, it could potentially freeze completely in the winter. The U.S. Army Corps of Engineers has performed a feasibility study for an infiltration gallery at the Galena AFS (appendix 6). To date, however, infiltration galleries have not been utilized at Galena. The Yukon River is a possible water source for Galena, but the cost of water treatment is significant. Flowing ice during freeze-up and break-up periods may damage or destroy water intake structures (Smith, 1986). The Yukon River represents a nearly inexhaustible supply of water for the village of Galena. The esti- 
mated water use for Galena is about $60,900 \mathrm{~L} / \mathrm{d}$. Mean annual flow of the Yukon River at Galena is about 4,600 $\mathrm{m}^{3} / \mathrm{s}$ (U.S. Geological Survey, 1957-76). This discharge is more than $6.5 \times 10^{6}$ times the water usage in the village of Galena and more than 360 times the quantity of water used in the entire State of Alaska in 1990 (Solley and others, 1993).

\section{Quality of Alternative Sources}

Alexander Lake may be a continuous source of water; however, no records of water quality data are available. Water quality of shallow arctic lakes in winter may decline as impurities such as salts and soluble inorganics become concentrated in the water under the ice (Smith, 1986).

The water quality of the Yukon River varies with the season and is generally better in the winter and than in the summer. Water from the Yukon River in winter is usually clear and concentrations of sediment are reduced. The high sediment concentrations in the Yukon River during the summer, however, would require treatment. Operation, development, and maintenance expenses would be high compared to those of existing supplies.

Water quality measured on the Yukon River at the Ruby gaging station is probably similar to the water quality at Galena. Analysis of water sample data for the period of record for the Yukon River at the Ruby station shows that iron content is typically below $0.30 \mathrm{mg} / \mathrm{L}$, but a peak of $1.86 \mathrm{mg} / \mathrm{L}$ was recorded on July 9, 1967 (appendix 7). Maximum and minimum sediment concentrations of $867 \mathrm{mg} / \mathrm{L}$ and $2 \mathrm{mg} / \mathrm{L}$ correlate with high and low river flow conditions (appendix 7).

\section{SUMMARY}

Galena serves as the transportation, government, and commercial center for the western Interior of Alaska and depends on the airport or the river for transportation. The subsistence lifestyle of the Native residents makes them dependent upon a sustainable environment. Frequent ice-jam flooding is hazardous to residents and their property. The ground-water flow is strongly influenced by the Yukon River and the continuity of permafrost in the area. The Yukon River could be an alternative drinking water supply, but may not be economical to develop and water intakes may be susceptible to damage from river ice flow. Local lakes that are deep enough not to freeze completely may also provide alternative sources of drinking water.

\section{REFERENCES CITED}

Aitken, G.W., 1963, Ground temperature observations, Galena, Alaska: U.S. Army Cold Regions Research and Engineering Laboratory, Corps of Engineers Technical Report 102, 15, p.

Alaska Department of Community and Regional Affairs, 1993, Community/borough map: Alaska Department of Community and Regional Affairs Municipal and Regional Assistance Division, 1 sheet.

Beltaos, Spyridon, 1990, Breakup jams, in Ryan, R.L., and Crissman, R.D., eds., Cold regions hydrology and hydraulics: American Society of Civil Engineers Monograph, p. 485-510.

Cass, J.T., 1959, Reconnaissance geologic map of the Nulato quadrangle, Alaska: U.S. Geological Survey Miscellaneous Investigations Geologic Map I-291, 1 sheet, scale, 1:250,000.

Cederstrom, D.J., Johnston, P.M., and Subitzky, S., 1953, Occurrence and development of ground water in permafrost regions: U.S. Geological Survey Circular 275, 30 p. 
Ecology and Environment, Inc., 1992, Environmental compliance investigation report, Galena FAA station Galena, Alaska: Anchorage [Draft report available from Federal Aviation Administration, Alaskan Region], variously paged.

Elias, M.M., and Vosburgh, R.M., 1946, Terrain and permafrost in the Galena area, Alaska: U.S. Geological Survey Permafrost Program Progress Report No. 1, 25 p.

Federal Emergency Management Agency, 1983, Flood insurance study, City of Galena, Alaska: U.S. Army Corps of Engineers, Alaska District, 17 p.

Ferrians, O.J. Jr., 1965, Permafrost map of Alaska: U.S. Geological Survey Miscellaneous Investigations Geologic Map I-445, 1 sheet (reprinted 1981).

Feulner, A.J., Childers, J.M., and Norman, V.W., 1971, Water resources of Alaska: U.S. Geological Survey Open-File Report, 60 p.

Fison and Associates, 1987, Galena community profile: Anchorage, Alaska, [Draft Report] 46 p.

Fountain, A.G., 1984, Yukon River breakup--The 82-year record: The Northern Engineer, v. 16, no. 1. p. 20-24.

Fountain, A.G., and Vaughn, B.H., 1984, Yukon River ice--Freeze-up data (1883-1975): U.S. Geological Survey Open-File Report 84-601, $51 \mathrm{p}$

Hartman, C.W., and Johnson, P.R., 1984, Environmental atlas of Alaska: University of Alaska Fairbanks, Institute of Water Resources/Engineering Experiment Station, 95 p.

Hopkins, D.M., Karlstrom, T.N.V., and others, 1955, Permafrost and groundwater in Alaska: U.S. Geological Survey Professional Paper 264-F, p. 113-146.

Jones, S.H., and Fahl, C.B., 1994, Magnitude and frequency of floods in Alaska and conterminous basins of Canada: U.S. Geological Survey Water-Resources Investigations Report 93-4179, 122 p., +2 pl

Leslie, L.D., 1989, Alaska climate summaries (2d ed.): University of Alaska Anchorage, Alaska Arctic Environmental Information and Data Center, Climate Center Technical Note 5.

Linsley, R.K., Kohler, M.A., and Paulhus, J.L., 1982, Hydrology for engineers (3d ed.): New York, McGraw Hill Book Company, 508 p.

McDonald M.G. and Harbaugh, A.W., 1988, A modular three-dimensional finite-difference ground-water flow model: U.S. Geological Survey Techniques of Water-Resources Investigations, book 6, chap. A1, 576 p.

Péwé, T.L., 1948, Terrain and permafrost of the Galena Air Base, Galena, Alaska: U.S. Geological Survey Permafrost Program Progress Report No. 7, 52 p.

Rieger, Samuel, Schoephorster, D.B., and Furbush, C.E., 1979, Exploratory soil survey of Alaska: Soil Conservation Service report, $213 \mathrm{p}$.

Smith, D.W., 1986, Cold climate utilities manual: Canadian Society for Civil Engineering, variously paged.

Solley, W.B., Pierce, R.R., and Perlman, H.A., 1993, Estimated use of water in the United States in 1990: U.S. Geological Survey Circular 1081, 76 p.

U.S. Army Corps of Engineers, 1983, Galena streambank protection, Galena, Alaska: U.S. Army Corps of Engineers, Alaska District, Section 14 Reconnaissance, 30 p.

1986, Bank stabilization Galena, Alaska: U.S. Army Corps of Engineers, Alaska District, Reconnaissance Feasibility Study, 69 p.

1987, Interim water resources study Yukon and Kuskokwim Rivers, Alaska: U.S. Army Corps of Engineers, Alaska District, 104 p.

1993, Alaskan communities flood hazard data: U.S. Army Corps of Engineers, Alaska District, 335 p.

U.S. Environmental Protection Agency, 1993, Drinking water regulations and health advisories: U.S. Environmental Protection Agency report, $11 \mathrm{p}$. 
U.S. Geological Survey, 1957-62, Quantity and quality of surface waters of Alaska. Annual reports as follows: water year 1957, Water-Supply Paper 1500; water year 1958, Water-Supply Paper 1570; water year 1959, Water-Supply Paper 1640; and water year 1960, Water-Supply Paper 1720.

1971, Surface-water supply of the United States, 1961-65, Part 15, Alaska: U.S. Geological Survey WaterSupply Paper 1936.

1976, Surface-water supply of the United States, 1966-70, Part 15, Alaska: U.S. Geological Survey WaterSupply Paper 2136.

1972-75, Water resources data for Alaska, 1971-74, Part 1, Surface water records: U.S. Geological Survey Water-Data Reports AK-71 to AK-74 (published annually).

1976-79, Water resources data for Alaska, water years 1975-78: U.S. Geological Survey Water-Data Reports AK-75-1 to AK-78-1 (published annually).

Viereck, L.A., and Little, E.L. Jr., 1972, Alaska trees and shrubs: U.S. Department of Agriculture Handbook No. 410, $265 \mathrm{p}$.

Wahrhaftig, Clyde, 1965, Physiographic divisions of Alaska: U.S. Geological Survey Professional Paper 482, 52 p.

Weber, F.L., and Péwé, T.L., 1970, Surficial and engineering geology of the central part of the Yukon-Koyukuk lowland, Alaska: U.S. Geological Survey Miscellaneous Investigations Geologic Map I-590, 2 sheets.

Williams, J.R., 1970, Ground water in permafrost regions of Alaska: U.S. Geological Survey Professional Paper 696, $83 \mathrm{p}$.

Williams, J.R., and Waller, R.M., 1963, Occurrence of ground water in the permafrost regions of Alaska: Purdue University, Indiana, International Conference on Permafrost, $19 \mathrm{p}$.

Woodward-Clyde Consultants, 1989, Installation restoration program, Phase II - confirmation/quantification, Stage 1, Campion, Fort Yukon, Galena, Indian Mountain, Murphy Dome, Cold Bay, Sparrevohn Air Force Stations, Alaska [final report]: Anchorage, Alaska, variously paged. 


\section{APPENDIX 1}

Data, assumptions, justifications, and data sources used in the MODFLOW packages 


\section{Ground-water flow at Galena, Alaska - Modflow Notes}

\section{BAS Package}

Packages Used: BAS, BCF, OC, PCG2, RCH, RIV, STR

- STR package not used in the Continuous permafrost model

Single-layer model

Grid size: 35 columns $x 40$ rows

IBOUND:

Continuous perfrost model run:

- cells south of RIV nodes set at no-flow (0)

- cells three to four cells north of RIV nodes set at no-flow (0)

- all other cells set at variable head (1)

No permafrost, low stream conductivity run:

- cells south of RIV nodes set at no-flow (0)

- all other cells set at variable head (1)

No permafrost, high stream conductivity run:

- cells south of RIV nodes set at no-flow (0)

- all other cells set at variable head (1)

Anisotropy: 1.00

\section{BCF Package}

Layer thickness: $200 \mathrm{ft}$ (-80 ft below MSL)

DELR: $1056 \mathrm{ft}$ (0.2 mile)

DELC: $1056 \mathrm{ft}$

Hydraulic Conductivity (K) along columns and rows: $80 \mathrm{ft} /$ day

RCH Package

Net annual recharge (recharge minus evapotranspiraton): $0.2300 \mathrm{E}-03 \mathrm{ft} / \mathrm{day}$ ( 1 inch/year)

\section{RIV Package}

River Reaches: 57

Slope of Bottom Elevation was obtained from U.S. Army Corps of Engineers (COE) (1981).

River Stage was estimated from USGS Nulato C-2, C-3, D-2, and D-3, 1:63,360 scale maps

River profile was extended by above and below the original COE (1981) survey

River Conductance: $0.3300 \mathrm{E}+08 \mathrm{ft} 2 /$ day $(\mathrm{K}=4.0 \mathrm{E}-02 \mathrm{~cm} / \mathrm{s})$, estimated

Reach Length (L): $1056 \mathrm{ft}$, unit cell size

Reach Width (W): 1056, unit cell size

Reach riverbed depth (D): $3 \mathrm{ft}$, estimated

Conductivity Equation: (LW/D)K

River Stage Height estimated to be $49 \mathrm{ft}$ higher than bottom elevation, COE (1981) 


\section{STR Package}

Stream modelled was Bear Creek, known locally as Crow Creek.

Assumed to be northward flowing

Assumed that upper reaches are affected by Yukon River stage height

Number of stream Reaches: 77

Number of stream Segments: 5

Streambed Conductance:

- low streambed conductance model value: $1.5 \mathrm{E} 02 \mathrm{ft}^{2} /$ day

- high steambed conductance model value: $1.5 \mathrm{E} 03 \mathrm{ft}^{2} /$ day

Reach Length (L): $1056 \mathrm{ft}$, unit cell size

Reach Width (W): 50, estimated

Reach streambed depth (D): $1 \mathrm{ft}$, estimated

Conductivity Equation: (LW/D)K

Stream slope: 0.00008

- estimated from USGS Nulato C-2, C-3, D-2, and D-3, 1:63,360 scale maps

Stream bed thickness: $0.5 \mathrm{ft}$, estimated

Stream bed bottom elevation: $0.5 \mathrm{ft}$ below streambed top elevation, estimated

Streambed top elevation: $4 \mathrm{ft}$ below stream stage, estimated

Stream stage: estimated from USGS Nulato C-2, C-3, D-2, and D-3, 1:63,360 scale maps 


\section{APPENDIX 2}

Example output file of the U.S. Geological Survey

Modular Finite-Difference Ground-Water Model 
U.S. GEOLOGICAL SURVEY MODULAR FINITE-DIFFERENCE GROUID-WATER MODEL

TWO DIMENSIONAL MODEL OF GROUND-WATER FLOW AT GALENA

1 LAYERS 40 ROWS 35 COLUMNS

1 STRESS PERIOD(S) IN SIMULATION

MODEL TIME UNIT IS DAYS

I 10 UNITS:

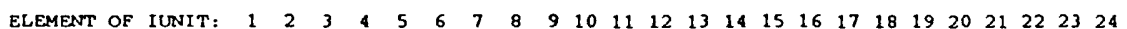

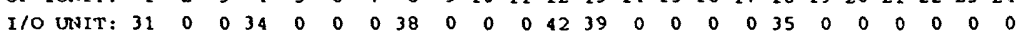

BAS1 - - BASIC MODEL PACKAGE, VERSION 1, 9/1/87 INPUT READ FROM UNIT 5

ARRAYS RHS AND BUFF WILL SHARE MEMORY.

START HEAD WILL BE SAVED

12679 ELEMENTS IN $X$ ARRAY ARE USED BY BAS

12679 ELEMENTS OF $X$ ARRAY USED OUT OF 350000

BCF2 -- BLOCK-CENTERED FLOW PACKAGE, VERSION 2, 7/1/91 INPUT READ FROM UNIT 31

STEADY-STATE SIMULATION

CELL-BY-CELL FLOWS WILL BE RECORDED ON UNIT 21

HEAD AT CELLS THAT CONVERT TO DRY $=0.00000 E+00$

WETTING CAPABILITY IS NOT ACTIVE

LAYER AQUIFER TYPE

1 1 1

2801 ELEMENTS IN $X$ ARRAY ARE USED BY BCF

15480 ELEMENTS OF $X$ ARRAY USED OUT OF 350000

RCII - - RECHARGE. PACKAGE, VERSION 1, 9/1/87 INPUT READ FROM UNIT 38

OPTION 1 - - RECHARGE TO TOP LAYER

CELL-BY-CELL FLOW TERMS WILL BE RECORDED ON UNIT 21

1400 ELEMENTS OF $X$ ARRAY USED FOR RECHARGE

16880 ELEMENTS OF $X$ ARRAY USED OUT OF 350000

RIV1 -- RIVER PACKAGE, VERSION 1, 9/1/87 INPUT READ FROM UN1T 34

MAXIMUM OF 57 RIVER NODES

CELL-BY-CELL FLOWS WILL BE RECORDED ON UIIT 21

342 ELEMENTS IN $X$ ARRAY ARE USED FOR RIVERS

17222 ELEMENTS OF $X$ ARRAY USED OUT OF 350000

PCG2 -- CONHUGATE GRADIENT SOLUTION PACKAGE, VERSION 2, 5/1/88

MAXIMUM OF 50 CALLS OF SOLUTION ROUTINE

MAXIMUM OF 10 INTERMAL ITERATIONS PER CALL TO SOLUTION ROUTINE

MATRIX PRECONDITIONING TYPE : 1

9600 ELEMEITS IN $X$ ARRAY ARE USED BY PCG

26822 ELEMENTS OF $X$ ARRAY USED OUT OF 350000

STRM - - STREAM PACKAGE, VERSION 2, 12/18/90 INPUT READ FROM UNIT 35

MAXIMUM OF 77 STREAM NODES

MUMBER OF STREAM SEGMENTS IS 5

NUMBER OF STREAM TRIBUTARIES IS 2

STREAM STAGES WILL BE CALCULATED USING A CONSTANT OF* * * * ***

1257 ELEMENTS IN $X$ ARRAY ARE USED FOR STREAMS

28079 ELEMENTS OF $X$ ARRAY USED OUT OF 350000

TWO DIMENSIONAL MODEL OF GROUND-WATER FLOW AT GALENA

1 layer, 40 rows, 35 columns, 0.2 mile grid

BOUNOARY ARRAY FOR LAYER 1 WILL BE READ ON UNIT 61 USING FORMAT: (35I2) 



\begin{tabular}{|c|c|c|c|c|c|c|c|c|c|c|}
\hline & 121.0 & 121.0 & 121.0 & 121.0 & 121.0 & 121.0 & 121.0 & 121.0 & 121.0 & 121.0 \\
\hline & 121.0 & 121.0 & 121.0 & 121.0 & 121.0 & 121.0 & 121.0 & 121.0 & 121.0 & 121.0 \\
\hline & 121.0 & 121.0 & 121.0 & 121.0 & 123.2 & & & & & \\
\hline \multirow[t]{4}{*}{9} & 121.0 & 121.0 & 121.0 & 121.0 & 121.0 & 121.0 & 121.0 & 121.0 & 121.0 & 121.0 \\
\hline & 121.0 & 121.0 & 121.0 & 121.0 & 121.0 & 121.0 & 121.0 & 121.0 & 121.0 & 121.0 \\
\hline & 121.0 & 121.0 & 121.0 & 121.0 & 121.0 & 121.0 & 121.0 & 121.0 & 121.0 & 121.0 \\
\hline & 121.0 & 121.0 & 121.0 & 121.0 & 123.2 & & & & & \\
\hline \multirow[t]{4}{*}{10} & 121.0 & 121.0 & 121.0 & 121.0 & 121.0 & 121.0 & 121.0 & 121.0 & 121.0 & 121.0 \\
\hline & 121.0 & 121.0 & 121.0 & 121.0 & 121.0 & 121.0 & 121.0 & 121.0 & 121.0 & 121.0 \\
\hline & 121.0 & 121.0 & 121.0 & 121.0 & 121.0 & 121.0 & 121.0 & 121.0 & 121.0 & 121.0 \\
\hline & 121.0 & 121.0 & 121.0 & 121.0 & 123.2 & & & & & \\
\hline \multirow[t]{4}{*}{11} & 121.0 & 121.0 & 121.0 & 121.0 & 121.0 & 121.0 & 121.0 & 121.0 & 121.0 & 121.0 \\
\hline & 121.0 & 121.0 & 121.0 & 121.0 & 121.0 & 121.0 & 121.0 & 121.0 & 121.0 & 121.0 \\
\hline & 121.0 & 121.0 & 121.0 & 121.0 & 121.0 & 121.0 & 121.0 & 121.0 & 121.0 & 121.0 \\
\hline & 121.0 & 121.0 & 121.0 & 121.0 & 123.2 & & & & & \\
\hline \multirow[t]{4}{*}{12} & 121.0 & 121.0 & 122.0 & 121.0 & 121.0 & 121.0 & 121.0 & 121.0 & 121.0 & 121.0 \\
\hline & 121.0 & 121.0 & 121.0 & 121.0 & 121.0 & 121.0 & 121.0 & 121.0 & 121.0 & 121.0 \\
\hline & 121.0 & 121.0 & 121.0 & 121.0 & 121.0 & 121.0 & 121.0 & 121.0 & 121.0 & 121.0 \\
\hline & 121.0 & 121.0 & 121.0 & 121.0 & 123.2 & & & & & \\
\hline \multirow[t]{4}{*}{13} & 121.0 & 121.0 & 121.0 & 121.0 & 121.0 & 121.0 & 121.0 & 121.0 & 121.0 & 121.0 \\
\hline & 121.0 & 121.0 & 121.0 & 121.0 & 121.0 & 121.0 & 121.0 & 121.0 & 121.0 & 121.0 \\
\hline & 121.0 & 121.0 & 121.0 & 121.0 & 121.0 & 121.0 & 121.0 & 121.0 & 121.0 & 121.0 \\
\hline & 121.0 & 121.0 & 121.0 & 121.0 & 123.2 & & & & & \\
\hline \multirow[t]{4}{*}{14} & 121.0 & 121.0 & 121.0 & 121.0 & 121.0 & 121.0 & 121.0 & 121.0 & 121.0 & 121.0 \\
\hline & 121.0 & 121.0 & 121.0 & 121.0 & 121.0 & 121.0 & 121.0 & 121.0 & 121.0 & 121.0 \\
\hline & 121.0 & 121.0 & 121.0 & 121.0 & 121.0 & 121.0 & 121.0 & 121.0 & 121.0 & 121.0 \\
\hline & 121.0 & 121.0 & 121.0 & 121.0 & 123.2 & 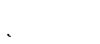 & & & & \\
\hline \multirow[t]{4}{*}{15} & 121.0 & 121.0 & 121.0 & 121.0 & 121.0 & 121.0 & 121.0 & 121.0 & 121.0 & 121.0 \\
\hline & 121.0 & 121.0 & 121.0 & 121.0 & 121.0 & 121.0 & 121.0 & 121.0 & 121.0 & 121.0 \\
\hline & 121.0 & 121.0 & 121.0 & 121.0 & 121.0 & 121.0 & 121.0 & 121.0 & 121.0 & 121.0 \\
\hline & 121.0 & 121.0 & 121.0 & 121.0 & 123.2 & & & & & \\
\hline \multirow[t]{4}{*}{16} & 121.0 & 121.0 & 121.0 & 121.0 & 121.0 & 121.0 & 121.0 & 121.0 & 121.0 & 121.0 \\
\hline & 121.0 & 121.0 & 121.0 & 121.0 & 121.0 & 121.0 & 121.0 & 121.0 & 121.0 & 121.0 \\
\hline & 121.0 & 121.0 & 121.0 & 121.0 & 121.0 & 121.0 & 121.0 & 121.0 & 121.0 & 121.0 \\
\hline & 121.0 & 121.0 & 121.0 & 121.0 & 123.2 & & & & & \\
\hline \multirow[t]{4}{*}{17} & 121.0 & 121.0 & 121.0 & 121.0 & 121.0 & 121.0 & 121.0 & 121.0 & 121.0 & 121.0 \\
\hline & 121.0 & 121.0 & 121.0 & 121.0 & 121.0 & 121.0 & 121.0 & 121.0 & 121.0 & 121.0 \\
\hline & 121.0 & 121.0 & 121.0 & 121.0 & 121.0 & 121.0 & 121.0 & 121.0 & 121.0 & 121.0 \\
\hline & 121.0 & 121.0 & 121.0 & 121.0 & 123.2 & & & & & \\
\hline \multirow[t]{4}{*}{18} & 121.0 & 121.0 & 121.0 & 121.0 & 121.0 & 121.0 & 121.0 & 121.0 & 121.0 & 121.0 \\
\hline & 121.0 & 121.0 & 121.0 & 121.0 & 121.0 & 121.0 & 121.0 & 121.0 & 121.0 & 121.0 \\
\hline & 121.0 & 121.0 & 121.0 & 121.0 & 121.0 & 121.0 & 121.0 & 121.0 & 121.0 & 121.0 \\
\hline & 121.0 & 121.0 & 121.0 & 121.0 & 123.2 & & & & & \\
\hline \multirow[t]{4}{*}{19} & 121.0 & 121.0 & 121.0 & 121.0 & 121.0 & 121.0 & 121.0 & 121.0 & 121.0 & 121.0 \\
\hline & 121.0 & 121.0 & 121.0 & 121.0 & 121.0 & 121.0 & 121.0 & 121.0 & 121.0 & 121.0 \\
\hline & 121.0 & 121.0 & 121.0 & 121.0 & 121.0 & 121.0 & 121.0 & 121.0 & 121.0 & 121.0 \\
\hline & 121.0 & 121.0 & 121.0 & 121.0 & 123.2 & & & & & \\
\hline 20 & 121.0 & 121.0 & 121.0 & 121.0 & 121.0 & 121.0 & 121.0 & 121.0 & 121.0 & 121.0 \\
\hline & 121.0 & 121.0 & 121.0 & 121.0 & 121.0 & 121.0 & 121.0 & 121.0 & 121.0 & 121.0 \\
\hline & 121.0 & 121.0 & 121.0 & 121.0 & 121.0 & 121.0 & 121.0 & 121.0 & 121.0 & 121.0 \\
\hline & 121.0 & 121.0 & 121.0 & 121.0 & 123.2 & & & & & \\
\hline 21 & 121.0 & 121.0 & 121.0 & 121.0 & 121.0 & 121.0 & 121.0 & 121.0 & 121.0 & 121.0 \\
\hline & 121.0 & 121.0 & 121.0 & 121.0 & 121.0 & 121.0 & 121.0 & 121.0 & 121.0 & 121.0 \\
\hline & 121.0 & 121.0 & 121.0 & 121.0 & 121.0 & 121.0 & 121.0 & 121.0 & 121.0 & 121.0 \\
\hline & 121.0 & 121.0 & 121.0 & 121.0 & 123.2 & & & & & \\
\hline 22 & 121.0 & 121.0 & 121.0 & 121.0 & 121.0 & 121.0 & 121.0 & 121.0 & 121.0 & 121.0 \\
\hline & 121.0 & 121.0 & 121.0 & 121.0 & 121.0 & 121.0 & 121.0 & 121.0 & 121.0 & 121.0 \\
\hline & 121.0 & 121.0 & 121.0 & 121.0 & 121.0 & 121.0 & 121.0 & 121.0 & 121.0 & 121.0 \\
\hline & 121.0 & 121.0 & 121.0 & 121.0 & 123.2 & & & & & \\
\hline 23 & 121.0 & 121.0 & 121.0 & 121.0 & 121.0 & 121.0 & 121.0 & 121.0 & 121.0 & 121.0 \\
\hline & 121.0 & 121.0 & 121.0 & 121.0 & 121.0 & 121.0 & 121.0 & 121.0 & 121.0 & 121.0 \\
\hline & 121.0 & 121.0 & 121.0 & 121.0 & 121.0 & 121.0 & 121.0 & 121.0 & 121.0 & 121.0 \\
\hline & 121.0 & 121.0 & 121.0 & 121.0 & 123.2 & & & & & \\
\hline 24 & 121.0 & 121.0 & 121.0 & 121.0 & 121.0 & 121.0 & 121.0 & 121.0 & 121.0 & 121.0 \\
\hline & 121.0 & 121.0 & 121.0 & 121.0 & 121.0 & 121.0 & 121.0 & 121.0 & 121.0 & 121.0 \\
\hline
\end{tabular}


$\begin{array}{lllll}121.0 & 121.0 & 121.0 & 121.0 & 123.2\end{array}$

HEAD PRINT FORMAT IS FORMAT NUMBER 3 DRAWDOWN PRINT FORMAT IS FORMAT NUMBER 3

HEADS WILL BE SAVED ON UNIT 20 DRAWDOWNS WILL BE SAVED ON UNIT 0

OUTPUT CONTROL IS SPECIFIED EVERY TIME STEP

COLUMN TO ROW ANISOTROPY $=1.000000$

\begin{tabular}{|c|c|c|c|c|c|c|c|c|c|}
\hline 1056.0 & 1056.0 & 1056.0 & 1056.0 & 1056.0 & 1056.0 & 1056.0 & 1056.0 & 1056.0 & 1056.0 \\
\hline 1056.0 & 1056.0 & 1056.0 & 1056.0 & 1056.0 & 1056.0 & 1056.0 & 1056.0 & 1056.0 & 1056.0 \\
\hline 1056.0 & 1056.0 & 1056.0 & 1056.0 & 1056.0 & 1056.0 & 1056.0 & 1056.0 & 1056.0 & 1056.0 \\
\hline 1056.0 & 1056.0 & 1056.0 & 1056.0 & 1056.0 & & & & & \\
\hline
\end{tabular}

$\begin{array}{lllll}1056.0 & 1056.0 & 1056.0 & 1056.0 & 1056.0\end{array}$

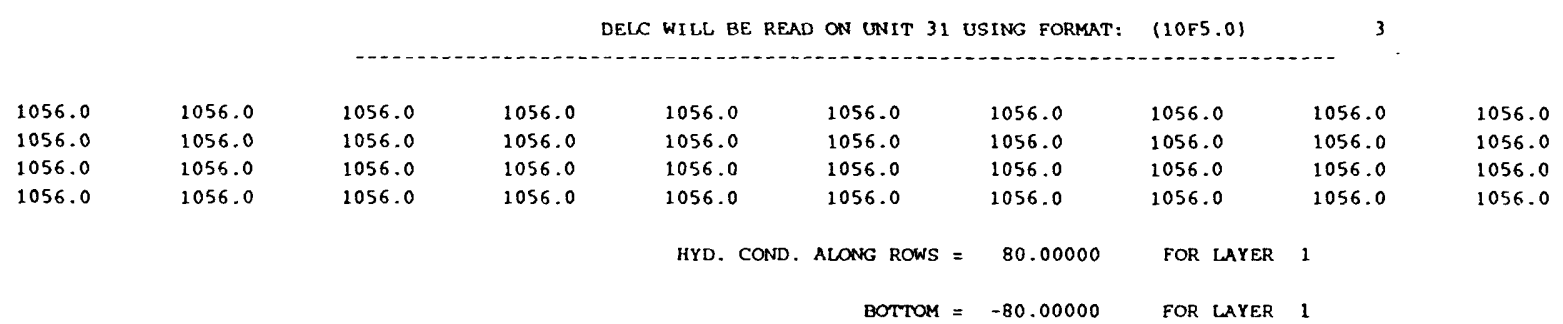

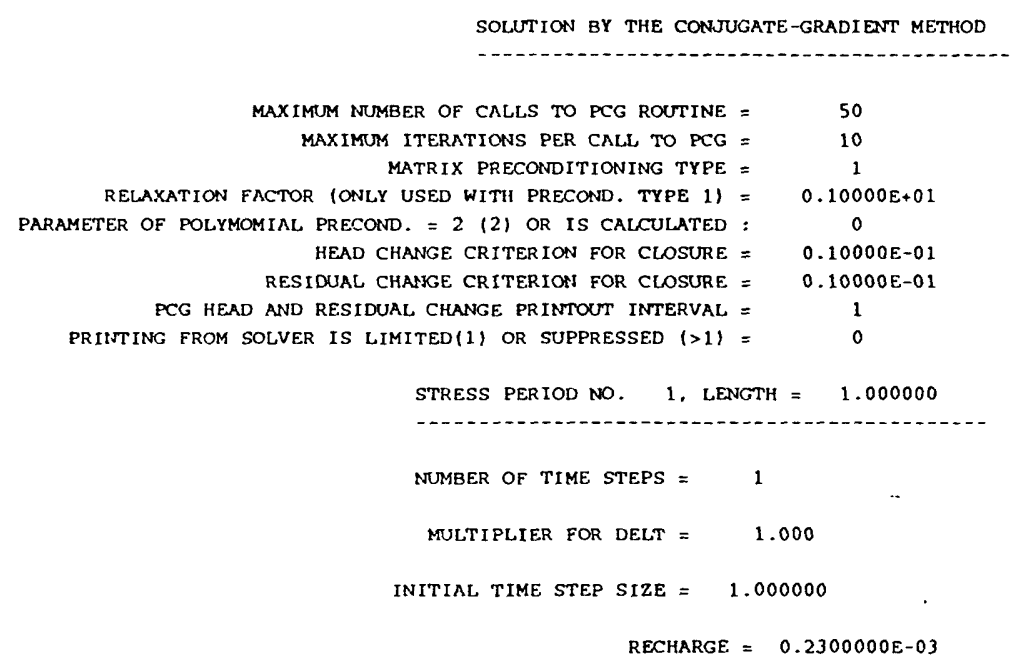

57 RIVER REACHES

\begin{tabular}{ccccccc} 
LAYER & ROW & COL & STAGE & CONDUCTANCE & BOTTOM ELEVATION & RIVER REACK \\
\hline 1 & 5 & 1 & 120.8 & $0.3300 \mathrm{E}+0 \mathrm{~B}$ & 71.80 & 1 \\
1 & 6 & 2 & 120.9 & $0.3300 \mathrm{O}+08$ & 71.90 & 2 \\
1 & 6 & 3 & 120.9 & $0.3300 \mathrm{E}+0 \mathrm{~B}$ & 71.90 & 3 \\
1 & 7 & 4 & 121.0 & $0.3300 \mathrm{E}+0 \mathrm{~B}$ & 72.00 & 4 \\
1 & 8 & 5 & 121.0 & $0.3300 \mathrm{E}+08$ & 72.00 & 5 \\
1 & 9 & 6 & 121.0 & $0.3300 \mathrm{O}+0 \mathrm{~B}$ & 72.00 & 6 \\
1 & 10 & 6 & 121.1 & $0.3300 \mathrm{E}+08$ & 72.10 & 7 \\
1 & 11 & 7 & 121.1 & $0.3300 \mathrm{E}+08$ & 72.10 & 8 \\
1 & 12 & 7 & 121.2 & $0.3300 \mathrm{E}+0 \mathrm{~B}$ & 72.20 & 9 \\
1 & 13 & 6 & 121.2 & $0.3300 \mathrm{E}+0 \mathrm{~B}$ & 72.20 & 10 \\
1 & 14 & 6 & 121.2 & $0.3300 \mathrm{E}+08$ & 72.20 & 11 \\
1 & 15 & 5 & 121.3 & $0.3300 \mathrm{E}+0 \mathrm{~B}$ & 72.30 & 12
\end{tabular}




\begin{tabular}{|c|c|c|c|c|c|c|c|c|c|}
\hline 1 & 17 & 17 & 2 & 9 & $0.0000 \mathrm{E}+00$ & 122.8 & 150.0 & 118.3 & 118.8 \\
\hline 1 & 16 & 17 & 2 & 10 & $0.0000 \mathrm{E}+00$ & 122.8 & 150.0 & 118.3 & 118.8 \\
\hline 1 & 16 & 16 & 2 & 11 & $0.0000 E+00$ & 122.7 & 150.0 & 118.2 & 118.7 \\
\hline 1 & 15 & 16 & 2 & 12 & $0.0000 \mathrm{E}+00$ & 122.6 & 150.0 & 118.1 & 118.6 \\
\hline 1 & 14 & 15 & 2 & 13 & $0.0000 \mathrm{E}+00$ & 122.5 & 150.0 & 118.0 & 118.5 \\
\hline 1 & 13 & 15 & 2 & 14 & $0.0000 \mathrm{E}+00$ & 122.4 & 150.0 & 117.9 & 118.4 \\
\hline 1 & 12 & 15 & 2 & 15 & $0.0000 E+00$ & 122.3 & 150.0 & 117.8 & 118.3 \\
\hline 1 & 11 & 15 & 2 & 16 & $0.0000 E+00$ & 122.2 & 250.0 & 117.7 & 118.2 \\
\hline 1 & 10 & 16 & 2 & 17 & $0.0000 \mathrm{E}+00$ & 122.2 & 150.0 & 117.7 & 118.2 \\
\hline 1 & 9 & 16 & 2 & 18 & $0.0000 E+00$ & 122.1 & 150.0 & 117.6 & 118.1 \\
\hline 1 & 8 & 16 & 2 & 19 & $0.0000 E+00$ & 122.0 & 150.0 & 117.5 & 118.0 \\
\hline 1 & 7 & 16 & 2 & 20 & $0.0000 E+00$ & 121.9 & 150.0 & 117.4 & 117.9 \\
\hline 1 & 7 & 16 & 3 & 1 & -1.000 & 121.9 & 150.0 & 117.4 & 117.9 \\
\hline 1 & 6 & 17 & 3 & 2 & $0.0000 \mathrm{E}+00$ & 121.8 & 150.0 & 117.3 & 117.8 \\
\hline 1 & 5 & 17 & 3 & 3 & $0.0000 E+00$ & 121.7 & 150.0 & 117.2 & 117.7 \\
\hline 1 & 4 & 17 & 3 & 4 & $0.0000 \mathrm{E}+00$ & 121.7 & 150.0 & 117.2 & 117.7 \\
\hline 1 & 3 & 17 & 3 & 5 & $0.0000 E+00$ & 121.6 & 150.0 & 117.1 & 117.6 \\
\hline 1 & 2 & 18 & 3 & 6 & $0.0000 E+00$ & 121.5 & 150.0 & 117.0 & 117.5 \\
\hline 1 & 21 & 34 & 4 & 1 & $0.0000 E+00$ & 123.8 & 150.0 & 119.3 & 119.8 \\
\hline 1 & 20 & 33 & 4 & 2 & $0.0000 E+00$ & 123.7 & 150.0 & 119.2 & 119.7 \\
\hline 1 & 19 & 32 & 4 & 3 & $0.0000 E+00$ & 123.6 & 150.0 & 119.1 & 119.6 \\
\hline 1 & 19 & 31 & 4 & 4 & $0.0000 \mathrm{E}+00$ & 123.5 & 150.0 & 119.0 & 119.5 \\
\hline 1 & 19 & 30 & 4 & 5 & $0.0000 \mathrm{E}+00$ & 123.4 & 150.0 & 118.9 & 119.4 \\
\hline 1 & 19 & 29 & 4 & 6 & $0.0000 \mathrm{E}+00$ & 123.3 & 150.0 & 118.8 & 119.3 \\
\hline 1 & 19 & 28 & 4 & 7 & $0.0000 E+00$ & 123.3 & 150.0 & 118.8 & 119.3 \\
\hline 1 & 19 & 27 & 4 & 8 & $0.0000 \mathrm{E}+00$ & 123.2 & 150.0 & 118.7 & 119.2 \\
\hline 1 & 19 & 26 & 4 & 9 & $0.0000 \mathrm{E}+00$ & 123.1 & 150.0 & 118.6 & 119.1 \\
\hline 1 & 19 & 25 & 4 & 10 & $0.0000 E+00$ & 123.0 & 150.0 & 118.5 & 119.0 \\
\hline 1 & 18 & 25 & 4 & 11 & $0.0000 \mathrm{E}+00$ & 122.9 & 150.0 & 118.4 & 118.9 \\
\hline 1 & 17 & 25 & 4 & 12 & $0.0000 E+00$ & 122.8 & 150.0 & 118.3 & 118.8 \\
\hline 1 & 16 & 25 & 4 & 13 & $0.0000 \mathrm{E}+00$ & 122.8 & 150.0 & 118.3 & 118.8 \\
\hline 1 & 15 & 25 & 4 & 14 & $0.0000 E+00$ & 122.7 & 150.0 & 118.2 & 118.7 \\
\hline 1 & 14 & 24 & 4 & 15 & $0.0000 E+00$ & 122.6 & 150.0 & 118.1 & 118.6 \\
\hline 1 & 13 & 24 & 4 & 16 & $0.0000 E+00$ & 122.5 & 150.0 & 118.0 & 118.5 \\
\hline 1 & 12 & 24 & 4 & 17 & $0.0000 E+00$ & 122.4 & 150.0 & 117.9 & 118.4 \\
\hline 1 & 11 & 24 & 4 & 18 & $0.0000 E+00$ & 122.3 & 150.0 & 117.8 & 118.3 \\
\hline 1 & 10 & 24 & 4 & 19 & $0.0000 E+00$ & 122.2 & 150.0 & 117.7 & 118.2 \\
\hline 1 & 9 & 24 & 4 & 20 & $0.0000 \mathrm{E}+00$ & 122.2 & 150.0 & 117.7 & 118.2 \\
\hline 1 & 8 & 23 & 4 & 21 & $0.0000 E+00$ & 122.1 & 150.0 & 117.6 & 118.1 \\
\hline 1 & 7 & 22 & 4 & 22 & $0.0000 E+00$ & 122.0 & 150.0 & 117.5 & 118.0 \\
\hline 1 & 6 & 21 & 4 & 23 & $0.0000 \mathrm{E}+00$ & 121.9 & 150.0 & 117.4 & 117.9 \\
\hline 1 & 5 & 20 & 4 & 24 & $0.0000 E+00$ & 121.8 & 150.0 & 117.3 & 117.8 \\
\hline 1 & 4 & 20 & 4 & 25 & $0.0000 E+00$ & 121.7 & 150.0 & 117.2 & 117.7 \\
\hline 1 & 4 & 19 & 4 & 26 & $0.0000 \mathrm{E}+00$ & 121.7 & 150.0 & 117.2 & 117.7 \\
\hline 1 & 3 & 18 & 4 & 27 & $0.0000 E+00$ & 121.6 & 150.0 & 117.1 & 117.6 \\
\hline 1 & 2 & 18 & 4 & 28 & $0.0000 \mathrm{E}+00$ & 121.5 & 150.0 & 117.0 & 117.5 \\
\hline 1 & 2 & 18 & 5 & 1 & -1.000 & 121.5 & 150.0 & 117.0 & 117.5 \\
\hline 1 & 1 & 18 & 5 & 2 & $0.0000 \mathrm{E}+00$ & 121.4 & 150.0 & 116.9 & 117.5 \\
\hline LAYER & ROW & $\cot$ & $\begin{array}{c}\text { SEGMENT } \\
\text { INתREER }\end{array}$ & $\begin{array}{l}\text { REACH } \\
\text { NUMBER }\end{array}$ & $\begin{array}{r}\text { STREAM } \\
\text { WIDTH }\end{array}$ & \multicolumn{2}{|r|}{ STREAM } & COEF & \\
\hline 1 & 24 & 10 & 1 & 1 & 50.00 & \multicolumn{2}{|r|}{$0.7800 \mathrm{E}-04$} & $0.3000 \mathrm{E}-01$ & \\
\hline 1 & 23 & 10 & 1 & 2 & 50.00 & \multicolumn{2}{|r|}{$0.7800 \mathrm{E}-04$} & $0.3000 E-01$ & \\
\hline 1 & 22 & 9 & 1 & 3 & 50.00 & & $0.7800 \mathrm{E}-04$ & $0.3000 E-01$ & - \\
\hline 1 & 21 & 9 & 1 & 4 & 50.00 & & $0.7800 \mathrm{E}-04$ & $0.3000 \mathrm{E}-01$ & \\
\hline 1 & 20 & 8 & 1 & 5 & 50.00 & & $0.7800 \mathrm{E}-04$ & $0.3000 E-01$ & \\
\hline 1 & 19 & 8 & 1 & 6 & 50.00 & & $0.7800 \mathrm{E}-04$ & $0.3000 E-01$ & \\
\hline 1 & 18 & 7 & 1 & 7 & 50.00 & & $0.7800 E-04$ & $0.3000 E-01$ & \\
\hline 1 & 17 & 7 & 1 & 8 & 50.00 & & $0.7800 \mathrm{E}-04$ & $0.3000 \mathrm{E}-01$ & \\
\hline 1 & 16 & 7 & 1 & 9 & 50.00 & & $0.7800 E-04$ & $0.3000 \mathrm{E}-01$ & . \\
\hline 1 & 15 & 8 & 1 & 10 & 50.00 & & $0.7800 \mathrm{E}-04$ & $0.3000 E-01$ & . \\
\hline 1 & 14 & 8 & 1 & 11 & 50.00 & & $0.7800 \mathrm{E}-04$ & $0.3000 E-01$ & $\cdot$ \\
\hline 1 & 13 & 9 & 1 & 12 & 50.00 & & $0.7800 \mathrm{E}-04$ & $0.3000 \mathrm{E}-01$ & \\
\hline 1 & 13 & 10 & 1 & 13 & 50.00 & & $0.7800 \mathrm{E}-04$ & $0.3000 \mathrm{E}-01$ & \\
\hline 1 & 13 & 11 & 1 & 14 & 50.00 & & $0.7800 \mathrm{E}-04$ & $0.3000 \mathrm{E}-01$ & \\
\hline 1 & 12 & 12 & 1 & 15 & 50.00 & & $0.7800 \mathrm{E}-04$ & $0.3000 E-01$ & \\
\hline 1 & 11 & 12 & 1 & 16 & 50.00 & & $0.7800 \mathrm{E}-04$ & $0.3000 \mathrm{E}-01$ & \\
\hline 1 & 10 & 13 & 1 & 17 & 50.00 & & $0.7800 \mathrm{E}-04$ & $0.3000 \mathrm{E}-01$ & \\
\hline 1 & 9 & 14 & 1 & 18 & 50.00 & & $0.7800 \mathrm{E}-04$ & $0.3000 \mathrm{E}-01$ & \\
\hline 1 & 9 & 15 & 1 & 19 & 50.00 & & $0.7800 E-04$ & $0.3000 \mathrm{E}-01$ & \\
\hline 1 & 8 & 16 & 1 & 20 & 50.00 & & $0.7800 \mathrm{E}-04$ & $0.3000 \mathrm{E}-01$ & \\
\hline 1 & 7 & 16 & 1 & 21 & 50.00 & & $0.7800 \mathrm{E}-04$ & $0.3000 \mathrm{E}-01$ & \\
\hline 1 & 25 & 22 & 2 & 1 & 50.00 & & $0.7800 \mathrm{E}-04$ & $0.3000 \mathrm{E}-01$ & \\
\hline 1 & 24 & 22 & 2 & 2 & 50.00 & & $0.7800 E-04$ & $0.3000 E-01$ & \\
\hline$i$ & 23 & 21 & 2 & 3 & 50.00 & & $0.7800 E-04$ & $0.3000 E-01$ & \\
\hline 1 & 22 & 21 & 2 & 4 & 50.00 & & $0.7800 E-04$ & $0.3000 \mathrm{E}-01$ & \\
\hline 1 & 21 & 20 & 2 & 5 & 50.00 & & $0.7800 E-04$ & $0.3000 \mathrm{E}-01$ & \\
\hline 1 & 20 & 19 & 2 & 6 & 50.00 & & $0.7800 \mathrm{E}-04$ & $0.3000 \mathrm{E}-01$ & \\
\hline 1 & 19 & 18 & 2 & 7 & 50.00 & & $0.7800 \mathrm{E}-04$ & $0.3000 \mathrm{E}-01$ & \\
\hline 1 & 18 & 17 & 2 & 8 & 50.00 & & $0.7800 \mathrm{E}-04$ & $0.3000 \mathrm{E}-01$ & \\
\hline
\end{tabular}




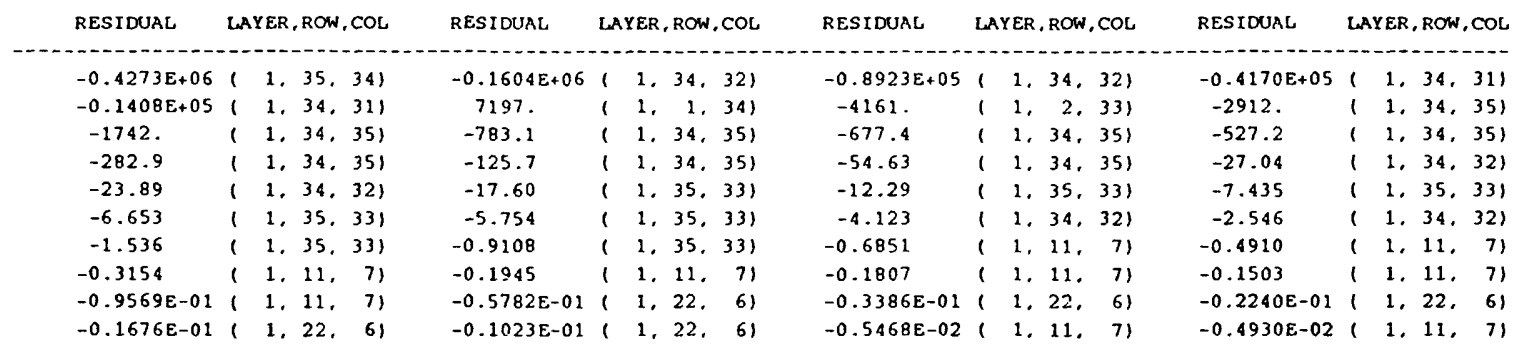

HEAD/DRAWDOWN PRINTOUT FLAG $=1$ TOTAL BUDGET PRINTOUT FLAG $=0 \quad$ CELL-BY - CELL FLOW TERM FLAG $=21$

OUTPUT FLAGS FOR ALL LAYERS ARE THE SAME:

HEAD DRAWDOWN HEAD DRAWDOWN

PRINTOUT PRINTOUT SAVE SAVE

1000

CONSTANT HEAD" BUDGET VALUES WILL BE SAVED ON UNIT 21 AT END OF TIME. STEP 1. STRESS PERIOD 1 - FLOW RIGHT FACE - BUDGET VALUES WILL BE SAVED ON UNIT 21 AT END OF TIME STEP 1 , STRESS PERIOD 1 "FLOW ERONT FACE - BUDGET VALUES WILL BE SAVED ON UNIT 21 AT END OF TIME STEP 1 , STRESS PERIOD 1 - Recharge" budget values will be saved on UNit 21 at end of time step 1 , Stress PERIOD 1

- river leakage* budget values will be saved on unit 21 at end of time step 1, stress period 1

\begin{tabular}{|c|c|c|c|c|c|c|c|c|}
\hline LAYER & ROW & COLUMN & $\begin{array}{l}\text { STREAM } \\
\text { NUMBER }\end{array}$ & $\begin{array}{l}\text { PEACH } \\
\text { RUMBER }\end{array}$ & $\begin{array}{l}\text { FLOW INTO } \\
\text { STREAM REACH }\end{array}$ & $\begin{array}{l}\text { FLOW INTO } \\
\text { AQUIFER }\end{array}$ & $\begin{array}{l}\text { FLOW OUT OF } \\
\text { STREAM REACH }\end{array}$ & $\begin{array}{l}\text { HEAD IN } \\
\text { STREAM }\end{array}$ \\
\hline 1 & 24 & 10 & 1 & 1 & $0.000 E+00$ & -408 & 408 & 119.60 \\
\hline 1 & 23 & 10 & 1 & 2 & 408 & -424 & 833 & 119.51 \\
\hline 1 & 22 & 9 & 1 & 3 & 833 & -414 & $0.125 E+04$ & 119.41 \\
\hline 1 & 21 & 9 & 1 & 4 & $0.125 E+04$ & -430 & $0.168 E+04$ & 119.31 \\
\hline 1 & 20 & 8 & 1 & 5 & $0.168 E+04$ & -405 & $0.208 E+04$ & 119.32 \\
\hline 1 & 19 & 8 & 1 & 6 & $0.208 E+04$ & -419 & $0.250 \varepsilon+04$ & 119.22 \\
\hline 1 & 18 & 7 & 1 & 7 & $0.250 E+04$ & -406 & $0.291 E+04$ & 119.12 \\
\hline 1 & 17 & 7 & 1 & 8 & $0.291 E+04$ & -414. & $0.332 E+04$ & 119.02 \\
\hline 1 & 16 & 7 & 1 & 9 & $0.332 E+04$ & -418 & $0.374 E+04$ & 118.92 \\
\hline 1 & 15 & 8 & 1 & 10 & $0.374 E+04$ & -451 & $0.419 E+04$ & 118.82 \\
\hline 1 & 14 & 8 & 1 & 11 & $0.419 E+04$ & -437 & $0.463 E+04$ & 118.83 \\
\hline 1 & 13 & 9 & 1 & 12 & $0.463 E+04$ & -481 & $0.511 E+04$ & 118.73 \\
\hline 1 & 13 & 10 & 1 & 13 & $0.511 E+04$ & -532. & $0.564 E+04$ & 118.63 \\
\hline 1 & 13 & 11 & 1 & 14 & $0.564 E+04$ & -582 & $0.622 E+04$ & 118.53 \\
\hline 1 & 12 & 12 & 1 & 15 & $0.622 E+04$ & -626 & $0.685 \varepsilon+04$ & 118.43 \\
\hline 1 & 11 & 12 & 1 & 16 & $0.685 F+04$ & -642 & $0.749 E+04$ & 118.34 \\
\hline 1 & 10 & 13 & 1 & 17 & $0.749 E+04$ & -688 & $0.818 E+04$ & 118.24 \\
\hline 1 & 9 & 14 & 1 & 18 & $0.818 E+04$ & -716 & $0.889 E+04$ & 118.24 \\
\hline 1 & 9 & 15 & 1 & 19 & $0.889 E+04$ & -754 & $0.965 E+04$ & 118.14 \\
\hline 1 & 8 & 16 & 1 & 20 & $0.965 E+04$ & -792 & $0.104 E+05$ & 118.04 \\
\hline 1 & 7 & 16 & 1 & 21 & $0.104 E+05$ & -808 & $0.112 E+05$ & 117.95 \\
\hline 1 & 25 & 22. & 2 & 1 & $0.000 \mathrm{E}+00$ & -614 & 614 & 119.51 \\
\hline 1 & 24 & 22 & 2 & 2 & 614 & -639 & $0.125 E+04$ & 119.41 \\
\hline 1 & 23 & 21 & 2 & 3 & $0.125 E+04$ & -651 & $0.190 E+04$ & 119.31 \\
\hline 1 & 22 & 21 & 2 & 4 & $0.190 \mathrm{E}+04$ & -660 & $0.256 E+04$ & 119.32 \\
\hline 1 & 21 & 20 & 2 & 5 & $0.256 E+04$ & -658 & $0.323 E+04$ & 119.22 \\
\hline 1 & 20 & 19 & 2 & 6 & $0.323 \varepsilon+04$ & -674 & $0.391 E+04$ & $\ldots 19.12$ \\
\hline 1 & 19 & 18 & 2 & 7 & $0.391 E+04$ & -676 & $0.458 E+04$ & 119.03 \\
\hline 1 & 18 & 17 & 2 & 8 & $0.458 E+04$ & -675 & $0.525 E+04$ & 118.93 \\
\hline 1 & 17 & 17 & 2 & 9 & $0.526 E+04$ & -691. & $0.595 E+04$ & 118.83 \\
\hline 1 & 16 & 17 & 2 & 10 & $0.595 E+04$ & -693 & $0.664 E+04$ & 118.83 \\
\hline 1 & 16 & 16 & 2 & 11 & $0.664 E+04$ & -686 & $0.733 E+04$ & 118.73 \\
\hline 1 & 15 & 16 & 2 & 12 & $0.733 E+04$ & -702 & $0.803 E+04$ & 118.64 \\
\hline 1 & 14 & 15 & 2 & 13 & $0.803 E+04$ & -694 & $0.872 E+04$ & 118.54 \\
\hline 1 & 13 & 15 & 2 & 14 & $0.872 E+04$ & -708 & $0.943 E+04$ & 118.44 \\
\hline 1 & 12 & 15 & 2 & 15 & $0.943 E+04$ & -722 & $0.102 E+05$ & 118.34 \\
\hline 1 & 11 & 15 & 2 & 16 & $0.102 E+05$ & -738 & $0.109 E+05$ & 118.24 \\
\hline 1 & 10 & 16 & 2 & 17 & $0.109 E+05$ & -763 & $0.117 E+05$ & 118.25 \\
\hline 1 & 9 & 16 & 2 & 18 & $0.117 E+05$ & -777 & $0.124 E+05$ & 118.15 \\
\hline 1 & 8 & 16 & 2 & 19 & $0.124 E+05$ & -791 & $0.132 E+05$ & 118.05 \\
\hline 1 & 7 & 16 & 2 & 20 & $0.132 E+05$ & -807 & $0.140 \varepsilon+05$ & 117.95 \\
\hline 1 & 7 & 16 & 3 & 1 & $0.253 E+05$ & -803 & $0.261 E+05$ & 117.98 \\
\hline 1 & 6 & 17 & 3 & 2 & $0.261 E+05$ & -848 & $0.269 E+05$ & 117.88 \\
\hline 1 & 5 & 17 & 3 & 3 & $0.269 E+05$ & -864 & $0.278 E+05$ & 117.78 \\
\hline 1 & 4 & 17 & 3 & 4 & $0.278 E+05$ & -865 & $0.287 E+05$ & 117.78 \\
\hline 1 & 3 & 17 & 3 & 5 & $0.287 E+05$ & -880 & $0.295 E+05$ & 117.68 \\
\hline 1 & 2 & 18 & 3 & 6 & $0.295 \varepsilon+05$ & -912. & $0.304 E+05$ & 117.58 \\
\hline 1 & 21 & 34 & 4 & 1 & $0.000 E+00$ & -705 & 705. & 119.81 \\
\hline 1 & 20 & 33 & 4 & 2 & 705 & -726 & $0.143 E+04$ & 119.71 \\
\hline
\end{tabular}




\begin{tabular}{|c|c|c|c|c|c|c|c|c|c|c|c|c|c|c|c|}
\hline \multirow[t]{3}{*}{12} & 999.0 & 999.0 & 999.0 & 999.0 & 999.0 & 999.0 & 121.2 & 121.6 & 121.9 & 122.2 & 122.4 & 122.6 & 122.8 & 123.0 & 123.2 \\
\hline & 123.3 & 123.5 & 123.7 & 123.8 & 124.0 & 124.1 & 124.2 & 124.3 & 124.4 & 124.5 & 124.6 & 124.7 & 124.8 & 124.9 & 125.0 \\
\hline & 125.0 & 125.1 & 125.1 & 125.1 & 125.1 & & & & & & & & & & \\
\hline \multirow[t]{3}{*}{13} & 999.0 & 999.0 & 999.0 & 999.0 & 999.0 & 121.2 & 121.4 & 121.7 & 121.9 & 122.2 & 122.4 & 122.6 & 122.8 & 123.0 & 123.2 \\
\hline & 123.3 & 123.5 & 123.7 & 123.8 & 124.0 & 124.1 & 124.2 & 124.3 & 124.4 & 124.5 & 124.6 & 124.7 & 124.8 & 124.9 & 124.9 \\
\hline & 125.0 & 125.0 & 125.1 & 125.1 & 125.1 & & & & & & & & & & \\
\hline \multirow[t]{3}{*}{14} & 999.0 & 999.0 & 999.0 & 999.0 & 999.0 & 121.2 & 121.5 & 121.7 & 122.0 & 122.2 & 122.4 & 122.7 & 122.8 & 123.0 & 123.2 \\
\hline & 123.3 & 123.5 & 123.7 & 123.8 & 123.9 & 124.1 & 124.2 & 124.3 & 124.3 & 124.4 & 124.6 & 124.6 & 124.7 & 124.8 & 124.9 \\
\hline & 124.9 & 125.0 & 125.0 & 125.0 & 125.0 & & & & & & & & & & \\
\hline \multirow[t]{3}{*}{15} & 999.0 & 999.0 & 999.0 & 999.0 & 121.3 & 121.4 & 121.6 & 121.8 & 122.1 & 122.3 & 122.5 & 122.7 & 122.9 & 123.0 & 123.2 \\
\hline & 123.3 & 123.5 & 123.6 & 123.8 & 123.9 & 124.0 & 124.1 & 124.2 & 124.3 & 124.4 & 124.5 & 124.6 & 124.7 & 124.7 & 224.8 \\
\hline & 124.9 & 124.9 & 124.9 & 125.0 & 125.0 & & & & & & & & & & \\
\hline \multirow[t]{3}{*}{16} & 999.0 & 999.0 & 999.0 & 121.3 & 121.4 & 121.5 & 121.7 & 121.9 & 122.1 & 122.3 & 122.5 & 122.7 & 122.9 & 123.0 & 123.2 \\
\hline & 123.3 & 123.5 & 123.6 & 123.8 & 123.9 & 124.0 & 124.1 & 124.2 & 124.3 & 124.3 & 124.4 & 124.5 & 124.6 & 124.7 & 124.7 \\
\hline & 124.8 & 124.8 & 124.9 & 124.9 & 124.9 & & & & & & & & & & \\
\hline \multirow[t]{3}{*}{17} & 999.0 & 999.0 & 121.3 & 121.9 & 121.5 &. .121 .6 & 121.8 & 122.0 & 122.2 & 122.3 & 122.5 & 122.7 & 122.9 & 123.0 & 123.2 \\
\hline & 123.3 & 123.4 & 123.6 & 123.7 & 123.9 & 124.0 & 124.1 & 124.2 & 124.2 & 124.3 & 124.4 & 124.5 & 124.5 & 124.6 & 224.7 \\
\hline & 124.7 & 124.8 & 124.8 & 124.8 & 124.8 & & & & & & & & & & \\
\hline \multirow[t]{3}{*}{18} & 999.0 & 999.0 & 121.4 & 121.4 & 121.5 & 121.7 & 121.8 & 122.0 & 122.2 & 122.4 & 122.5 & 122.7 & 122.9 & 123.0 & 123.2 \\
\hline & 123.3 & 123.4 & 123.6 & 123.7 & 123.8 & 123.9 & 124.0 & 124.1 & 124.2 & 124.2 & 124.3 & 124.4 & 124.5 & 124.5 & 124.6 \\
\hline & 124.6 & 124.7 & 124.7 & 124.7 & 124.8 & & & & & & & & & & \\
\hline \multirow[t]{3}{*}{19} & 999.0 & 999.0 & 999.0 & 121.4 & 121.6 & 121.7 & 121.9 & 122.0 & 122.2 & 122.4 & 122.6 & 122.7 & 122.9 & 123.0 & 123.2 \\
\hline & 123.3 & 123.4 & 123.5 & 123.7 & 123.8 & 123.9 & 124.0 & 124.1 & 124.1 & 124.2 & 124.3 & 124.3 & 124.4 & 124.5 & 124.5 \\
\hline & 124.6 & 124.6 & 124.6 & 124.7 & 124.7 & & & & & & & & & & \\
\hline \multirow[t]{3}{*}{20} & 999.0 & 999.0 & 999.0 & 121.5 & 121.6 & 121.7 & 121.8 & 122.0 & 122.2 & 122.4 & 122.6 & 122.7 & 122.9 & 123.0 & 123.2 \\
\hline & 123.3 & 123.4 & 123.5 & 123.6 & 123.7 & 123.8 & 123.9 & 124.0 & 124.1 & 124.2 & 124.2 & 124.3 & 124.4 & 124.4 & 124.5 \\
\hline & 124.5 & 124.5 & 124.6 & 124.6 & 124.6 & & & & & & & & & & \\
\hline \multirow[t]{3}{*}{21} & 999.0 & 999.0 & 999.0 & 999.0 & 121.5 & 121.6 & 121.8 & 127.0 & 122.2 & 122.4 & 122.5 & 122.7 & 122.9 & 123.0 & 123.1 \\
\hline & 123.3 & 123.4 & 123.5 & 123.6 & 123.7 & 123.8 & 123.9 & 124.0 & 124.0 & 124.1 & 124.2 & 124.2 & 124.3 & 124.4 & 124.4 \\
\hline & 124.4 & 124.5 & 124.5 & 124.5 & 124.5 & & & & & & & & & & \\
\hline \multirow[t]{3}{*}{22} & 999.0 & 999.0 & 999.0 & 999.0 & 999.0 & 121.5 & 121.8 & 122.0 & 122.2 & 122.4 & 122.5 & 122.7 & 122.8 & 123.0 & 123.1 \\
\hline & 123.2 & 123.4 & 123.5 & 123.5 & 123.6 & 123.7 & 123.8 & 123.9 & 124.0 & 124.1 & 124.1 & 124.2 & 124.2 & 124.3 & 124.3 \\
\hline & 124.4 & 124.4 & 124.4 & 124.4 & 124.4 & & & & & & & & & & \\
\hline 23 & 999.0 & 999.0 & 999.0 & 999.0 & 999.0 & 121.6 & 121.8 & 122.0 & 122.2 & 122.3 & 122.5 & 122.7 & 122.8 & 123.0 & $12 ? .1$ \\
\hline & 123.2 & 123.3 & 123.4 & 123.5 & 123.6 & 123.7 & 123.7 & 123.8 & 123.9 & 124.0 & 124.0 & 124.1 & 124.2 & 124.2 & 124.3 \\
\hline & 124.3 & 124.3 & 124.3 & 124.4 & 124.4 & & & & & & & & & & \\
\hline 24 & 999.0 & 999.0 & 999.0 & 999.0 & 999.0 & 121.6 & 121.8 & 122.0 & 122.1 & 122.3 & 122.5 & 122.7 & 122.8 & 122.9 & 123.1 \\
\hline & 123.2 & 123.3 & 123.4 & 123.5 & 123.5 & 123.6 & 123.7 & 123.8 & 123.8 & 123.9 & 124.0 & 124.0 & 124.1 & 124.1 & 124.2 \\
\hline & 124.2 & 124.2 & 124.3 & 124.3 & 124.3 & & & & & & & & & & \\
\hline 25 & 999.0 & 999.0 & 999.0 & 999.0 & 999.0 & 999.0 & 121.7 & 121.9 & 122.1 & 122.3 & 122.5 & 122.6 & 122.8 & 122.9 & 123.0 \\
\hline & 123.1 & 123.2 & 123.3 & 123.4 & 123.5 & 123.5 & 123.6 & 123.7 & 123.7 & 123.8 & 123.9 & 123.9 & 124.0 & 124.0 & 124.1 \\
\hline & 124.1 & 124.1 & 124.2 & 124.2 & 124.2 & & & & & & & & & & \\
\hline 26 & 999.0 & 999.0 & 999.0 & 999.0 & 999.0 & 999.0 & 121.7 & 121.9 & 122.1 & 122.3 & 122.5 & 122.6 & 122.7 & 122.9 & 123.0 \\
\hline & 123.1 & 123.2 & 123.2 & 123.3 & 123.4 & 123.5 & 123.5 & 123.6 & 123.7 & 123.7 & 123.8 & 123.8 & 123.9 & 123.9 & 124.0 \\
\hline & 124.0 & 124.0 & 124.1 & 124.1 & 124.1 & & & & & & & & . & & \\
\hline 27 & 999.0 . & 999.0 & 999.0 & 999.0 & 999.0 & 999.0 & 121.7 & 121.9 & 122.1 & 122.3 & 122.4 & 122.6 & 122.7 & 122.8 & 122.9 \\
\hline & 123.0 & 123.1 & 123.2 & 123.2 & 123.3 & 123.4 & 123.4 & 123.5 & 123.5 & 123.6 & 123.7 & 123.7 & 123.8 & 123.8 & 123.8 \\
\hline & 123.9 & 123.9 & 123.9 & 124.0 & 124.0 & & & & & & & & & & \\
\hline 28 & 999.0 & 999.0 & 999.0 & 999.0 & 999.0 & 999.0 & 121.8 & 122.0 & 122.1 & 122.3 & 122.4 & 122.5 & 122.7 & 122.8 & 122.9 \\
\hline & 122.9 & 123.0 & 123.1 & 123.2 & 123.2 & 123.3 & 123.3 & 123.4 & 123.4 & 123.5 & 123.5 & 123.6 & 123.6 & 123.7 & 123.7 \\
\hline & 123.8 & 123.8 & 123.8 & 123.8 & 123.9 & & & & & & & & & & \\
\hline 29 & 999.0 & 999.0 & 999.0 & 999.0 & 999.0 & 121.8 & 121.9 & 122.0 & 122.1 & 122.3 & 122.4 & 122.5 & 122.6 & 122.7 & 122.8 \\
\hline & 122.9 & 122.9 & 123.0 & 123.1 & 123.1 & 123.2 & 123.2 & 123.2 & 123.3 & 123.3 & 123.4 & 123.4 & 123.5 & 123.5 & 123.6 \\
\hline & 123.6 & 123.7 & 123.7 & 123.7 & 123.7 & & & & & & & & & & \\
\hline 30 & 999.0 & 999.0 & 999.0 & 999.0 & 999.0 & 121.8 & 121.9 & 122.0 & 122.1 & 122.3 & 122.4 & 122.5 & 122.6 & 122.6 & 122.7 \\
\hline & 122.8 & 122.8 & 122.9 & 122.9 & 123.0 & 123.0 & 123.1 & 123.1 & 123.1 & 123.2 & 123.2 & 123.3 & 123.3 & 123.4 & 123.4 \\
\hline & 123.5 & 123.5 & 123.6 & 123.6 & 123.6 & & & & & & & & & & \\
\hline 31 & 999.0 & 999.0 & 999.0 & 999.0 & 999.0 & 121.9 & 121.9 & 122.0 & 122.1 & 122.2 & 122.3 & 122.4 & 122.5 & 122.6 & 122.6 \\
\hline & 122.7 & 122.7 & 122.8 & 122.8 & 122.9 & 122.9 & 122.9 & 122.9 & 123.0 & 123.0 & 123.1 & 123.1 & 123.1 & 123.2 & 123.3 \\
\hline & 123.3 & 123.4 & 123.4 & 123.5 & 123.5 & & & & & & & & & & \\
\hline 32 & 999.0 & 999.0 & 999.0 & 999.0 & 999.0 & 999.0 & 121.9 & 122.0 & 122.1 & 122.2 & 122.3 & 122.3 & 122.4 & 122.5 & 122.5 \\
\hline
\end{tabular}




\title{
APPENDIX 3
}

\author{
U.S. Army Corps of Engineers
}

"Report on rehabilitation of well no. 1 water supply well, Galena AP" 
REPORT ON REHABILITATION OF

WELL NO. I - WATER SUPPLY WELL

GALENA AP: .. .

Corps of Engineers

U. S. Army Engineer District, Alaska Anchorage, Alaska

Prepared by

Foundations and Materials Branch

31 October 1963 
REPORT ON REHABILITATION OF

WELL NO. 1 - WATER SUPPLY WELL

GALENA AP

\section{REFERENCE:}

Directive No. NPD-2, Job No. Galena AP-64-MRA \& MC-AP, dated

25 July 1963.

2. SCOPE OF REPORT:

This report covers the rehabilitation and subsequent testing of

Well No. I at Galena AP. Well No. 1 had not been in use for several years, because of á reported but unproved contamination by petroleum products, with the result that the base water supply system was completely dependent on one well (Well No. 2 at Bldg 1578, the water treatment building). In order to eliminate this dependence on a single well, it was decided to rehabilitate and reactivate Well No. 1. Rehabilitation of the well was authorized as set forth in Par. 1 above, and the rehabilitation work was performed by personnel of the Foundations and Materials Branch, USAED Alaska.

\section{DESCRIPTION OF WELL:}

Well No. I is located at coordinates N 101,575; E 98,265, with an original ground elevation of approximately $120 \mathrm{ft}$ MSL. The well is in the southeast corner of a lean-to structure on the east side of the fire station, the F.F. elevation of the lean-to being within a few inches of the original ground elevation. Access to the well is attained through a hatch in the roof of the lean-to. The well was drilled in 1944 under the direction of the CAA (now FAA), and was cased throughout its entire depth with 6-inch diameter casing. The total depth of the well was 
reported to be $210 \mathrm{ft}$, and the old CAA records Indicate that a well screen was scheduled for installation at the bottom of the well. No records could be found pertaining to the extent of development of the well, but the well was reported to have been test-pumped at a rate of $13 \mathrm{gpm}$ with a drawdown of $0.8 \mathrm{ft}$. At the time the rehabilitation work was started the well was equipped with a Jacuzzl jet pump, type 75-T-44M, ser1al No. 50290, with a $7.5 \mathrm{HP}$ electric motor (220 V, 3 phase, 1800 RPM). This pump had been installed in the well at some time prior to 1954. The electric wires to the pump installation had been removed at some time in the past, and there was no way of activating the pump motor without extending new electric lines to the well location.

\section{PRELIMINARY PUMPING TEST:}

The Alaska Air Command had requested that rehabilitation of the well comence by performing a 72-hour pumping test with the old Jacuzzl jet pump, in ordex to determine the extent of fuel-oil contamination, if any, of the water in the aquifer zone, and to determine if the specific yield of the well was sufficiently high and the degree of contamination sufficiently low to render feasible any further efforts at rehabilitation. Therefore, an electric power line was extended to the well, the pump activated, and a pumping test was made. Pumping was started at 10:00 a.m. 7 June 1963 and continued steadily for 70 hours until 8:00 a.m. 10 June 1963. Pumping was carried on at a rate of $46 \mathrm{gpm}$, the maximum production rate of which the Jacuzzi pump was capable, and the drawdown at this rate was found to be about 3 feet. Details of the pumping test are set forth in Table 1. The pumping rate was determined by collecting the discharge water in a container of known capacity and measuring the length of time 
required to fill the container. Water levels were determined by means of an electric water level indicator. The discharge water was found at the beginning of the pumping test to be free of any fuel ofl contamination, and no trace of any petroleum product appeared in the discharge water.at any time during the test. Three water samples were collected during the course of the pumping test and were analyzed in the laboratory for total solids and for total iron content. Details of these chemical tests are set forth below:

$\begin{array}{lcc}\text { Sample No. } & \text { Total Solids } & \text { Total Iron, as Fe } \\ \text { 1, at start of pump test } & 776 \mathrm{ppm} & 140.0 \mathrm{ppm} \\ \text { 2, after } 24 \mathrm{hr} \text { pumping } & 298 \mathrm{ppm} & 4.40 \mathrm{ppm} \\ 3 \text {, after } 30 \mathrm{hr} \text { pumping } & 212 \mathrm{ppm} & 4.68 \mathrm{ppm} .\end{array}$

The high inftial values for total solids and total fron are a reflection of the fact that the water column in the unused well was standing essentially stagnant for a number of years. Three water samples were collected during the pumping test by the Base Medical Techniclan and were tested for bacteriological contamination, the first sample being collected immediately at the beginning of the pumping test. The rather limited tests performed by the Base Medical Technician were negative, indicating that the well water was free from harmful bacteria. The pumping test demonstrated that the water was uncontaminated, that it was essentially the same in total solids and iron content as the water from Well No. 2, the current base supply well, and that the specific yield of the well was sufficiently high to justify a complete rehabilitation of the well and installation of a larger pump. 


\section{REHABILITATION OF WELL:}

The Jacuzzi jet pump was removed from the well through the hatch In the roof 17 and $18 \mathrm{July} 1963$, and the well was explored for forelgn objects. It was evident from the results of the preliminary pumping test that little or no sand had collected at the bottom of the well. Nuts, bolts, and miscellaneous small items of hardware, sufficient to fill a small sack, ware removed from the bottom of the well 19 July 1963, together with a number of large pebbles. No significant amount of sand was found. No screen was encountered in the well, and the bottom of the casing was found by measurement to be $205 \mathrm{ft}$ below the lean-to floor. The casing was scrubbed with strong detergent throughout its entire depth; in scrubbing the bottom portion of the casing there was some indication that the lowermost $8 \mathrm{ft}$ of the casing might be perforated. Seven and a half pounds of dry chlorine were introduced into the well and allowed to stand overnight. On 20 July 1963 the chlorine solution was bailed from the well, the well was surged and beiled vigorously, and the casing was swabbed, until only Insignificant amounts of sand, rust, and scale could be extracted from the well. The total amount of material removed from the well was 8 mall in bulk, and the cleaning and developing of the well was considered to be complete. After completion of development, the static water level was measured at $8.6 \mathrm{ft}$ below the lean-to floor level. On 20-22 July 1963 a Myers $5 \mathrm{HP}$ submersible pump (60 cycle, 3 phase, 220 volt) was placed in the well as a permanent installation, the water intake being set at a depth of $76 \mathrm{ft}$ below the top of the casing. An air line for a permanent air-pressure drawdown gauge was installed at the same time, the lower end of the alr line being set at $73 \mathrm{ft}$ below the top of the casing. 


\section{DESCRIPTION OF FINAL TESTS:}

The Myers submersible pump installed in the well was used in making the final pumping test. Pumping was begun at 1:30 p.m. 24 July. 1963 and continued steadily for nineteen hours until 8:30 a.m. 25 July 1963, at which time the pumping test was considered complete and the pumping was stopped. Pumping was carried on at a rate of $130 \mathrm{gpm}$. The pumping rate was determined by collecting the discharge water in a container of known capacity and measuring the length of time required to fill the container. Water levels were determined by means of the installed air line and a pressure gauge. Just before pumping was stopped, a water sample was obtained for chemical analysis.

\section{RESULTS OF TESTS:}

The static water level was determined to be $8.0 \mathrm{ft}$ below the floor level immediately before pumping started. The drawdown level at the $130 \mathrm{gpm}$ pumping rate assumed a depth of $18.0 \mathrm{ft}$ below the floor level a. few moments after pumping had been started, and remained stable at that depth for the duration of the pumping test. Upon stopping the pump, the water level returned to static level immediately. A drawdown curve for the well, based on both the final pumping test and the preliminary pumping test, is shown on Chart 1. The water sample collected at the end of the pumping test for chemical analysis was tested in the laboratory, and the details of the chemical analysis are shown on Table 2. The discharge water cleared . almost immediately after pumping started, and remained clear throught the duration of the pumping test.

\section{CONCLUSIONS:}

The tests show that the water from the well was not contaminated by either petroleum products or bacteria, and that the rehabilitated well 
will produce potable water at a rate of $130 \mathrm{gpm}$ with a drawdown of approximately $10 \mathrm{ft}$. The well is an excellent producer, and has the capability to produce water considerably in excess of the capacity of the Myers submersible pump installed as a permanent pump. This well is able to meet by itself the demands on the base water supply system. The water has an iron content only slightly less than that of the water from the well which currently furnishes the base water supply, and the same type of water treatment for Iron is applicable to the water from both wells.

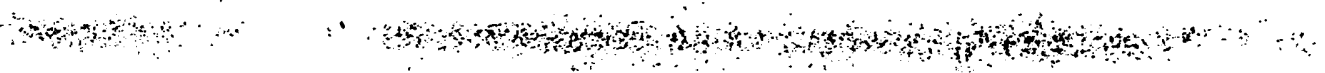


TABLE 1

TEST DATA

PRELIMINARY PUMPING TEST

WELL-NO..1 - 6-INCH DIA. WATER SUPPLY WELL

GALENA AP

Gal per Min Depth in Ft to

Date \& Time Pumped Water Level

Remarks

7 June $63-1000$

1001

1100

1200

1300

1400

1500

1800

2000

2100

8 June $63-0600$

0700

0800

0900

1000

1100

1200

1300

1400

1500

1600

10 June $63-0800$
5.3

46

7.3
Static water level - started pumping at $46 \mathrm{gpm}$ rate, discharge water momentarily greenish color, clearing rapidly, no sand. Discharge water clearing. Collected water sample for determination of total solids and iron content. Collected water sample for bacteriological testing.

Dfscharge water clear.

7.3

7.5

7.8

$46 \quad 7.8$

$46 \quad \cdot \quad 7.5$

Collected water sample for bacteriological testing.

7.5

7.6

7.5

7.8

46

\section{$-46$}

7.8

7.9

8.0

8.3

8.4

Collected water sample for determination of total solids and iron content. Collected water sample for bacteriological testing.

$\begin{array}{ll}46 & 8.2 \\ 46- & 8.3 \\ 46 & 8.2 \\ 46 & 8.2 \\ 46 & 8.3 \\ 46 & 8.3\end{array}$

Collected water sample for chemical analysis.

46 Not measured Stopped pumping - pump test complete. 
CONTRACT NO. DA- EXP

DE IAB FILE NO. 1988-63
REPORT DATE: 28 Aug 63

SUBMITTERS SAMPLB NO.

SOURCE: Galena AFS. Fire Station Well, Sampled after 19 hours of pumping at a rate of $130 \mathrm{gpm}$. Sampled $0830 \mathrm{hrs} 25 \mathrm{July} 63$. Permanent Water Well No. 1. SAMPLE \& IABEL:

REQUEST: Hater Analysis

TEST RESULTS:

$\mathrm{pH}--------------6.92$

Conductivity - - at $27^{\circ} C_{-}-\ldots--1-$

431 mmhos

Total solfds - - - - - - - - - - - -

283

Calcium, as $\mathrm{Ca}-\ldots$

ppm

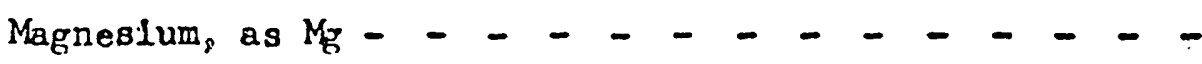

$\mathrm{ppm}$

Sodium \& Potassium, as $\mathrm{Na}$ -

ppm

Total Iron, as Fo - - - - - - - - -

$\mathrm{ppm}$

Organic Iron, as Fo

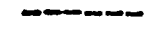

ppm

Manganese, as Mn -

\begin{tabular}{cc}
\hline 40.0 & $\mathrm{ppm}$ \\
0.4 & $\mathrm{ppm}$ \\
1.0 & $\mathrm{ppm}$ \\
7.0 & $\mathrm{ppm}$ \\
226 & $\mathrm{ppm}$ \\
0.0 & $\mathrm{ppm}$
\end{tabular}

Alkalinity, Phenolphthaloin, as $\mathrm{CaCO}_{3}-\ldots$

Total hardness, as $\mathrm{CaCO}_{3}$ - - - - - - - - - -

ppm

Carbonate hardness, as $\mathrm{CaCO}_{3}-$ - - - - - - - -

Non-Carbonate hardne8s, as $\mathrm{CaCO}_{3}-\ldots-\ldots$

ppm

ppm

Freo Carbon Dloxddo, as $\mathrm{CO}_{2}-\cdots-\ldots$

ppm

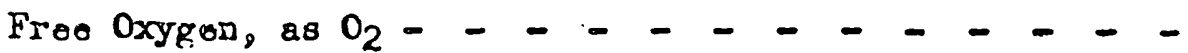

ppm

REMARKS:

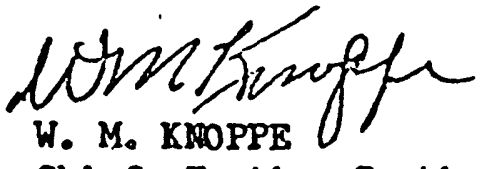




\section{CHART I}

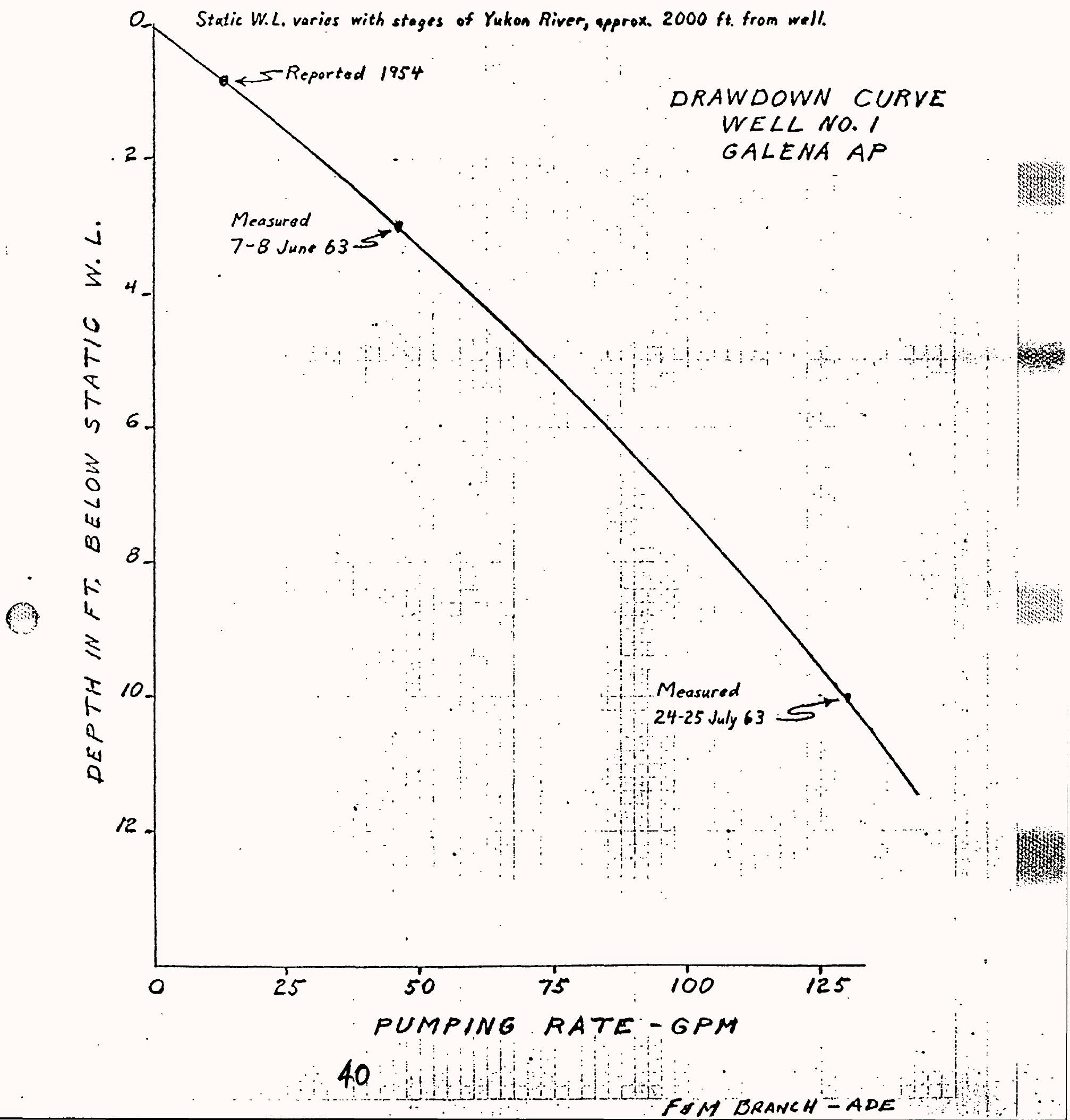




\section{APPENDIX 4}

Selected well drillers' logs, aquifer test data, and ground-water quality data for Galena

from U.S. Public Health Service village files 
NELL LOG

U.S. PUBLIC HEALTH SERVICE, DIVISION OE INDIAN HEALTH

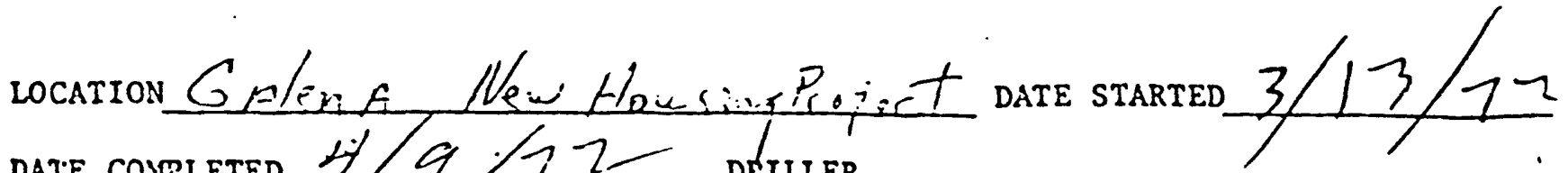
DATE CONBLETED H/9/72 DrILLER

TOTAL DEPTH OF WELL $\angle 223$ FT. CASING INSTALLED $\angle 273$ DIAMETER $6 "$

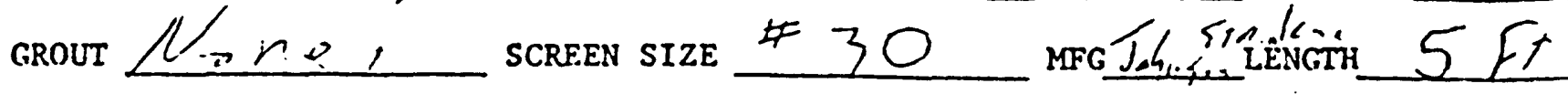
StATIC WATER LEVEL 40 HRS. PUREED 24026 GPS. DRAWDOWN 28 FT

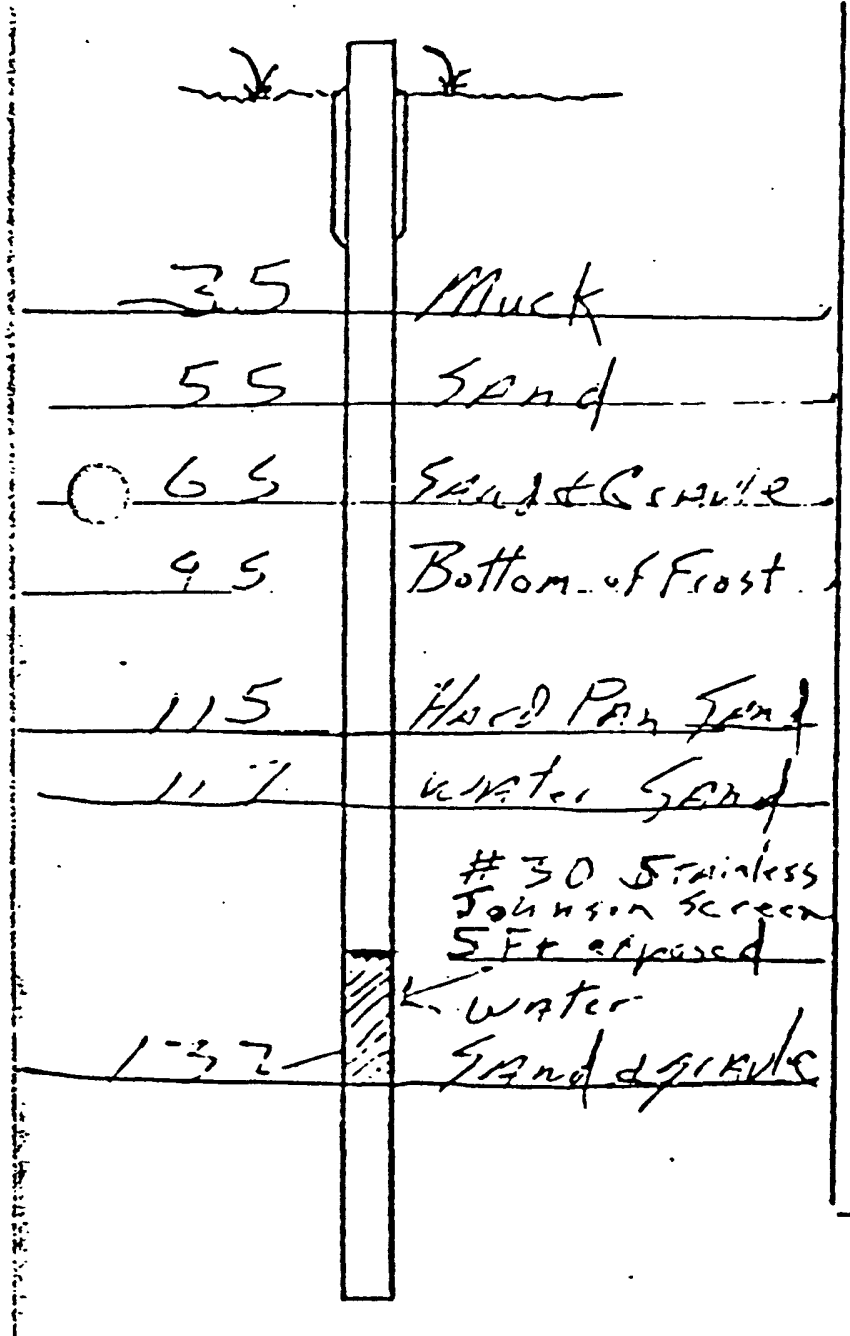

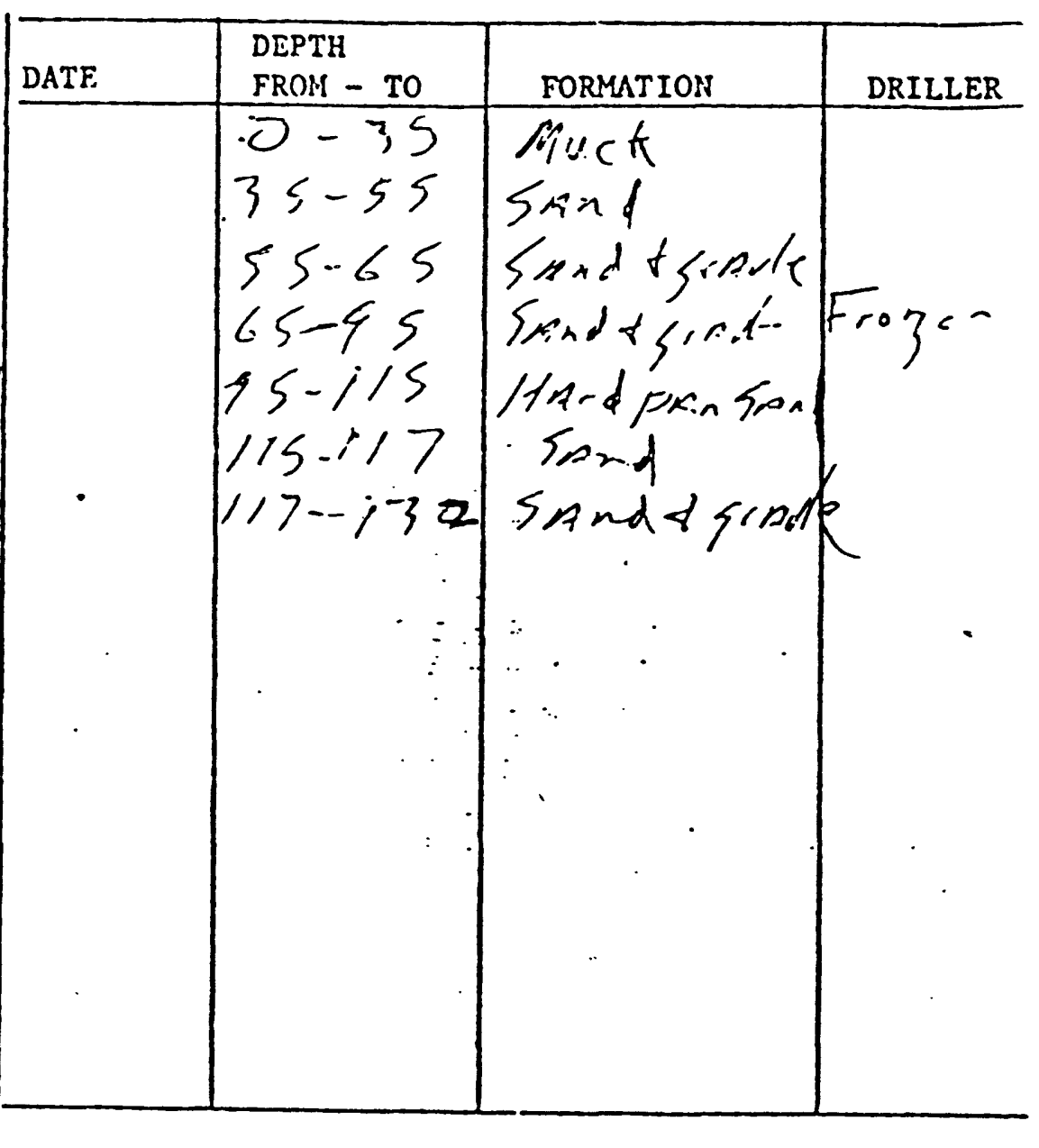

SPECIAL NOTES:

Fist. $0-45$

(j)

42 
WELL LOG

U.S. PUELIC MEILTH SERVICL, DIVISIC:: OF ISDIA: HEALTH

LOCATIO: GALENA, ALASKS:

DATE STARTED 6-18-74

DATE CO:TLITED 7-5-74

DRILLTR.

Frinter

TOTAL DEPTH OF IELL 147 FT. CAST::G I:STALIEA 142 DIAIETER 6"

GroUI SCRTE: SIZF. 40 :IF i[i:CTi:

$5 "$

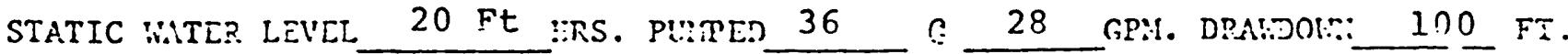

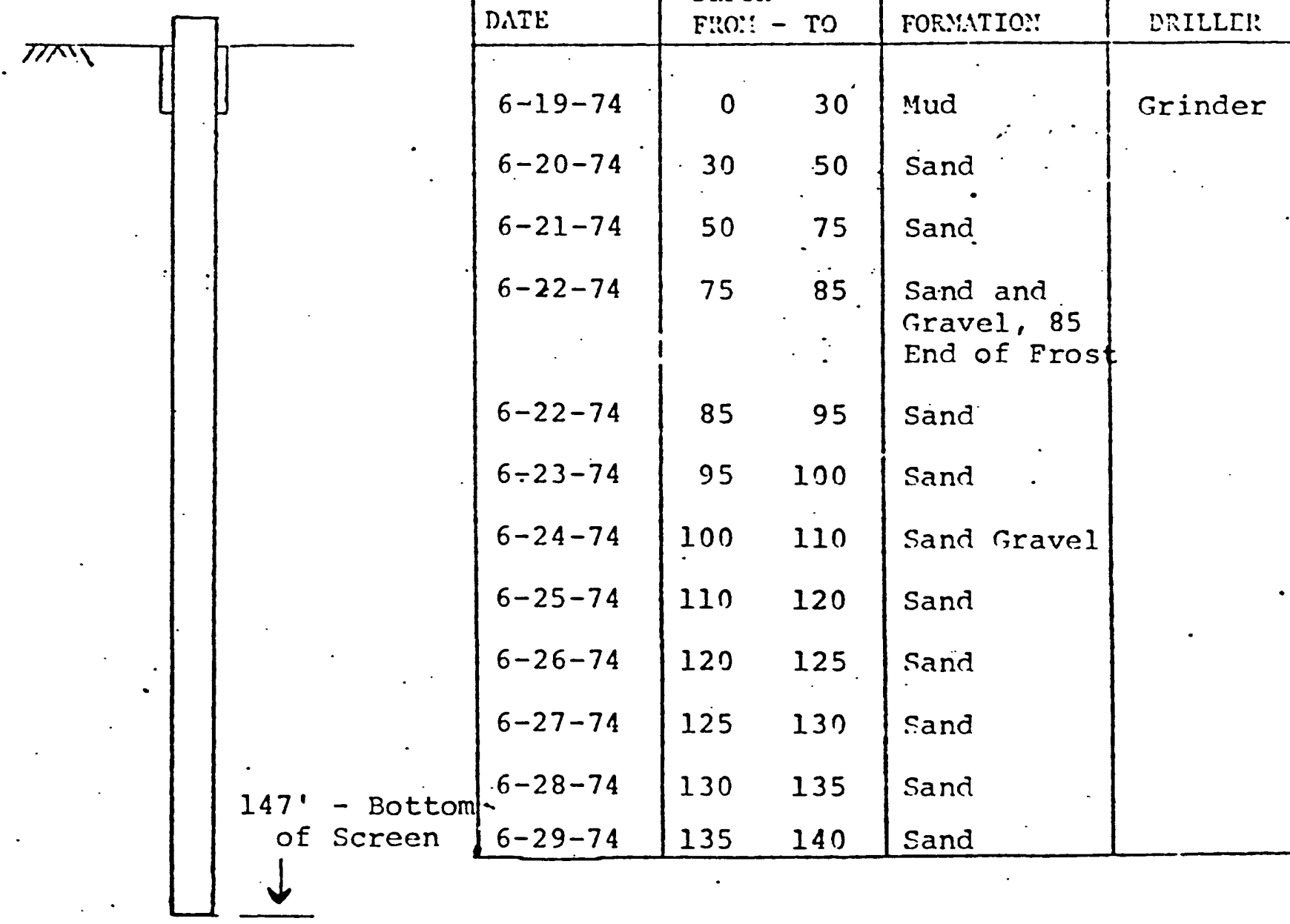

SPECIAL :OTES: End of frost $85 \mathrm{Ft}$ water level $20 \mathrm{Ft}$ from ground Pump Set 143 level screen set from $142^{\prime}$ to $147^{\prime}$. 


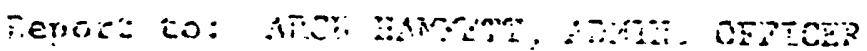

Cistor.

?. C. 5 n $7-74 j$

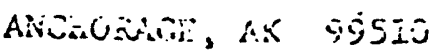

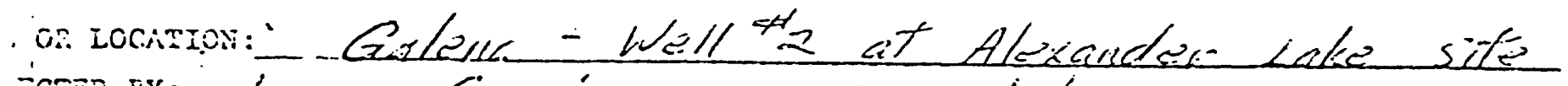

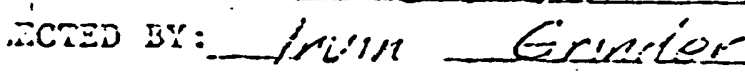
TITER SYSTES iNTL $7 / 8 / 24$ :

HeP SYSTES

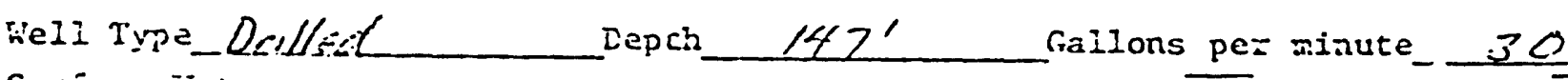

Suríäce iñiser: Taporary $I$ Permanent I!

riumber or inores Seirved:

Treatmear: 17 ies $\mathbb{T}$ No jow or ixisting source

PLPPOSE OF AYAIYSIS

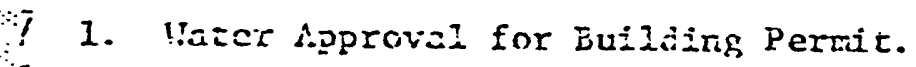

(Colunn 1)

2. Routine Azaiysis.

(Coluran I\& 2)

?. 3. Special: Checl. Specific Items for Analysis

(Columns $1,2,3$ )

Corusa: 2

COLLAN 2

conuts: 3

\begin{tabular}{|c|c|c|c|c|c|}
\hline & Aralysis & Limits & & Anal. & \multirow{3}{*}{$\frac{\text { Loimit }}{125}$} \\
\hline (Fej & 4.98 & 0.3 & \multirow{2}{*}{$\begin{array}{l}\text { tagnesium } \\
\text { (Mig) }\end{array}$} & & \\
\hline -ire $\because$ & $\therefore .17$ & 17. & & 30 & \\
\hline$\because=i \mathrm{ie}(\mathrm{C} I)$ & 7 & 1250 & \multirow{2}{*}{$\begin{array}{l}\text { Valcium } \\
(C n)\end{array}$} & \multirow{2}{*}{49} & \multirow[t]{2}{*}{300} \\
\hline$\left(P 0_{1}\right)$ & \multirow[b]{2}{*}{$Q .53$} & .059000 & & & \\
\hline suhaze & & 30 oror & TurbiuiEv & 300 & $i$ \\
\hline \multirow[t]{2}{*}{ al iiarciness } & \multirow[b]{2}{*}{174} & iso sofi & \multirow{4}{*}{ 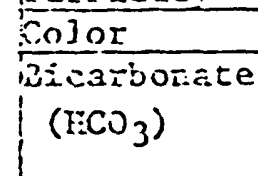 } & \multirow[t]{2}{*}{5} & 15 \\
\hline & & 300 hazd & & & 25 \\
\hline \multirow[t]{3}{*}{$\neq x$ rents } & $\underbrace{\prime \prime}_{1}$ & 10 & & \multirow{2}{*}{$2 \times 4$} & 5 no \\
\hline & \multirow[b]{2}{*}{7.65} & 6.5 & & & \multirow{2}{*}{ 1000r } \\
\hline & & 5.5 & \multirow{3}{*}{$\frac{\text { Cartonate }}{\text { Alkalirity }}$} & \multirow{2}{*}{$\frac{0}{192}$} & \\
\hline \multirow{2}{*}{$\begin{array}{l}\text { cific } \\
\text { ductasise }\end{array}$} & \multirow{2}{*}{$3 \% 3$} & & & & 350 \\
\hline & & & & \multirow[b]{2}{*}{214} & \multirow[b]{2}{*}{500} \\
\hline$\therefore 110$ & & . & $\begin{array}{l}\text { solved } \\
\text { Solics }\end{array}$ & & \\
\hline
\end{tabular}

ITENS :

STELTIO:S :

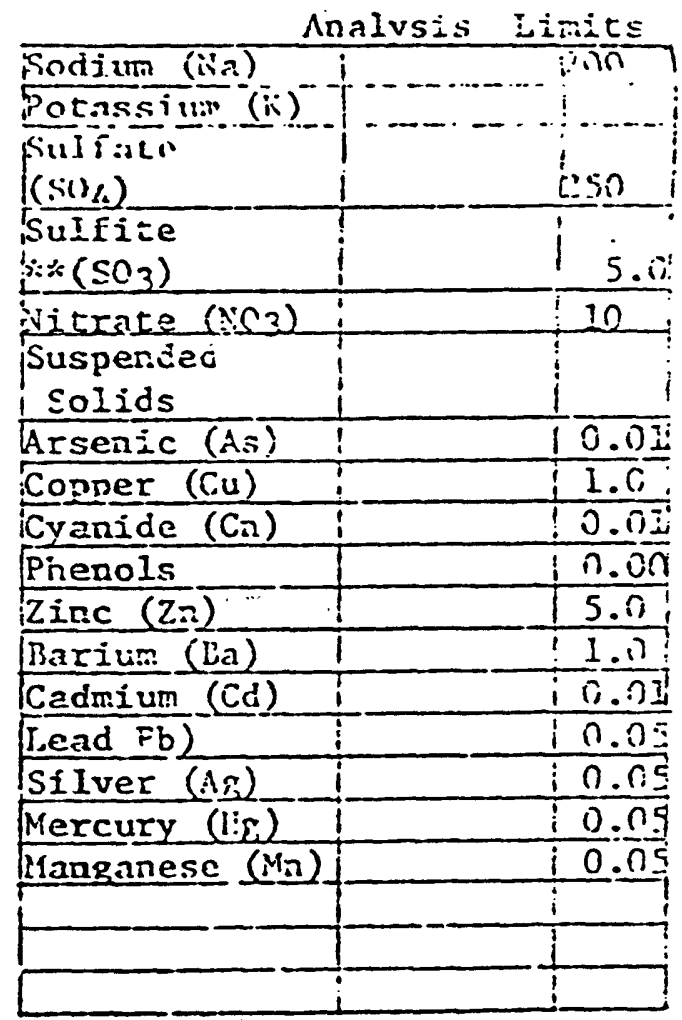

Rinse container several times in water source to be sampled. Plice ca? on sarpic container firmly.

Flace sample in carton mailer, and forward to: Fublic llealth laboratory sRo, !ledical Nrts BIdf. Fouch $J$ Juneau, Ak yysoi 

oject No.

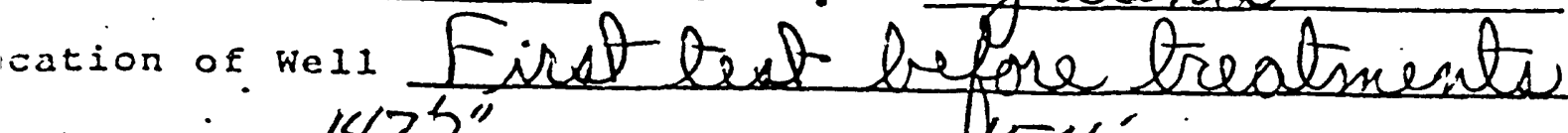

pth of well/472"ft. Length of casing 54 ft. Pumped well/ observation well Observation Well, Dist. to Pumped Well $32 ; 5 \%$ te Drilling Completed

Driller ft. Top of casing to static Leve: ock Elapsed Time nue Since Pumping started/stopped

$\left[\begin{array}{l|l}\text { Depth to } & \begin{array}{l}\text { Drawdown } \\ \text { Water } \\ \text { From toc }\end{array} \\ \text { Recovery } & \text { Reciock } \\ \text { Rime }\end{array} \mid\right.$

gpen Date rested $10-7-2$ \& Elapsed Time bepth to Drawdowr Since Pumping fiater Started/stonpedFrom TOC Recover

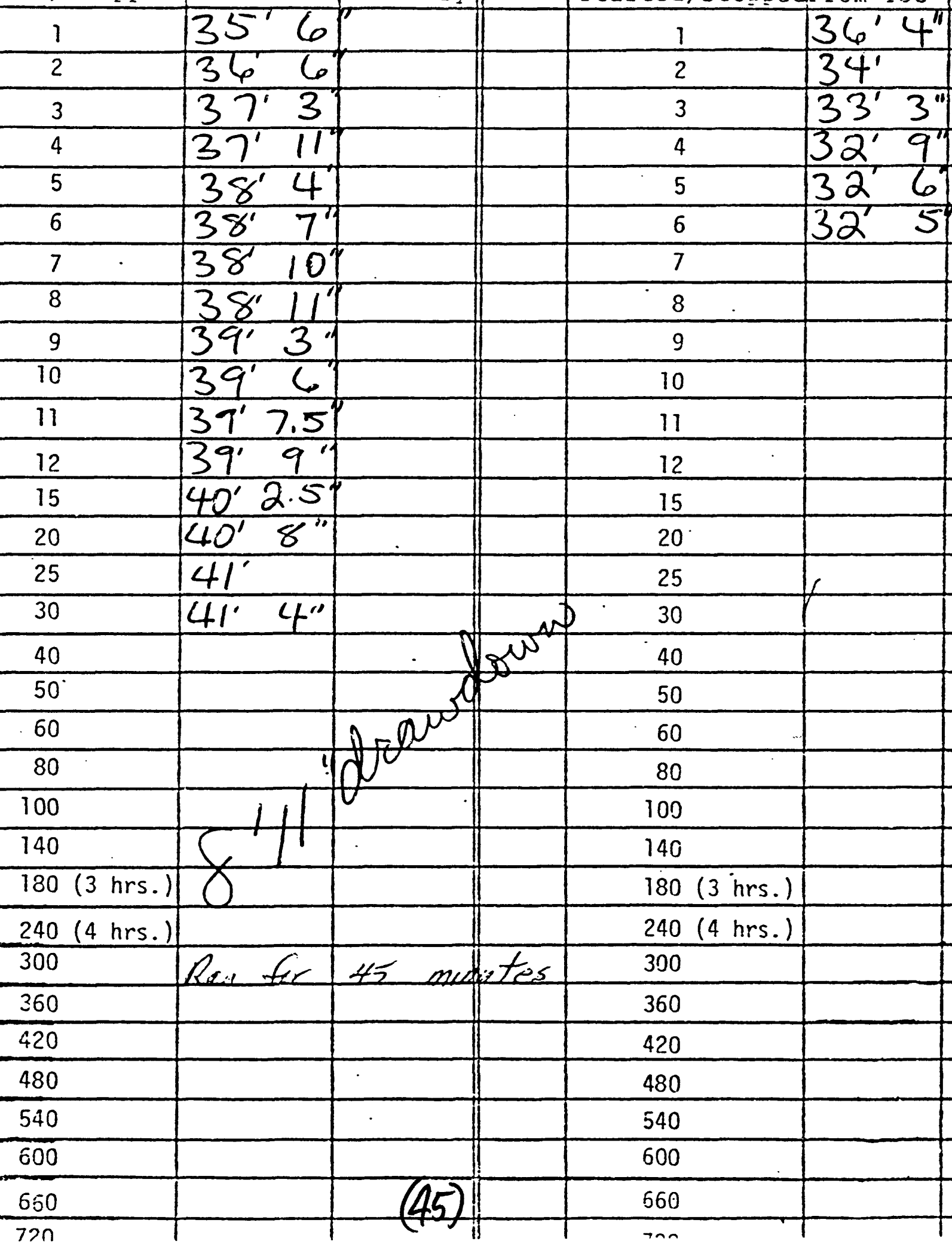


$\operatorname{page} \alpha$ of ject no.

ation of we11 Lecond te after istacid " ci

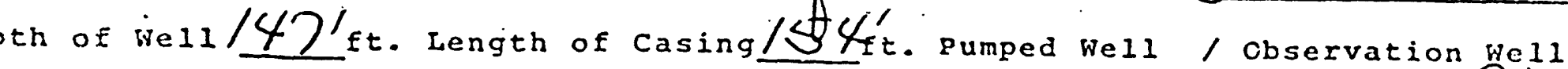
Observation Well, Dist. to Pumped Well ft. Top of casing to static Leve? : Drilling Completed

\section{Drilier logem.)} Depth to $\left|\begin{array}{lr}\text { Drawdown } \\ \text { Dr }\end{array}\right|$-gom lock Water From TOC Recovery

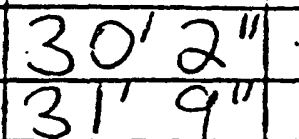

3

4

5

6

7

8

9

10

11

12

15

20

25

30

40

50

60

80

100

140

180 (3 hrs.)

240 (4 hrs.)

300

360

420

430

540

500

550
Elapsea rime Tepen to Drawdows Date rested $10: i \quad i$ Elapsed Time pepth to since pumping fiater started/stoppedFrom Toc Recovers

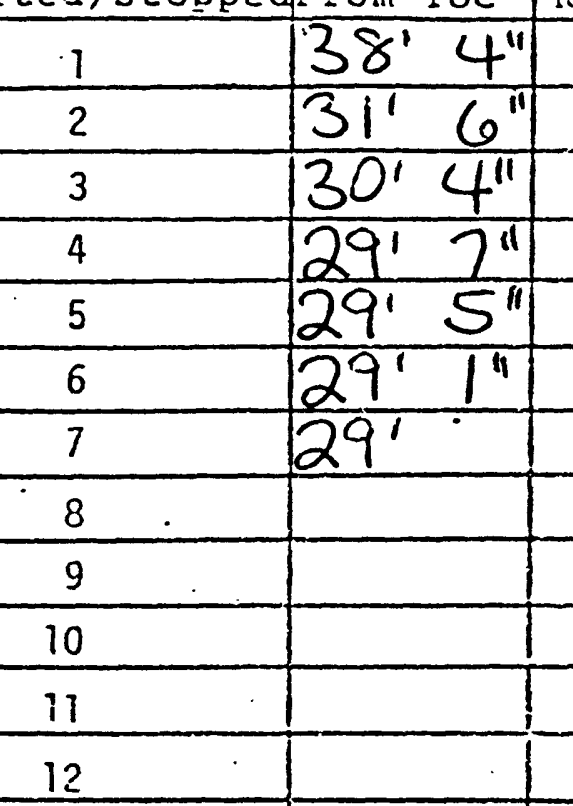
15 20 25 30 40 50 60 80

100

140

180 (3 hrs.)

240 ( 4 hrs.)

300

360

420

480

540

600

E.EO 
ject No.

ation of well

th of well 17 ft. Length of casing

observation Well, Dist. to Pumped Well

Drilling Completed

Driller

ck Elapsed Time

since Pumping

started/stopped

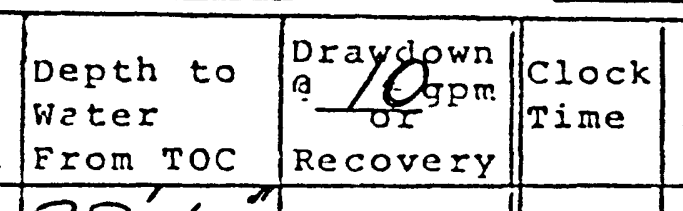

ft. Pumped Well / Observation Well $34^{\prime} 5^{\prime \prime} k$ ft. Top of casing to static leve Date Tested $10-42-3$

Elapsed ine Drawdow: Elapsed Time pepth to Drawdow Since Pumping fiater tor started/stoppedErom ToC Recover

\begin{tabular}{|l|l||l|}
1 & $37^{\prime} 6$ & \\
\hline 2 & $37^{\prime} 10^{\prime \prime}$ & \\
\hline 3 & 1 &
\end{tabular}

\begin{tabular}{l|l|l}
1 & $34^{\prime} 8$ & \\
\hline 2 & $34^{\prime} 5$ & \\
\hline 3 & & \\
\hline 4 & & \\
\hline 5 & & \\
\hline 6 & & \\
\hline 7 & & \\
\hline 8 & & \\
\hline 9 & & \\
\hline 10 & & \\
\hline 11 & & \\
\hline 12 & & \\
\hline 15 & & \\
\hline
\end{tabular}


Galena Wellname $7-5-74$ prege of 5

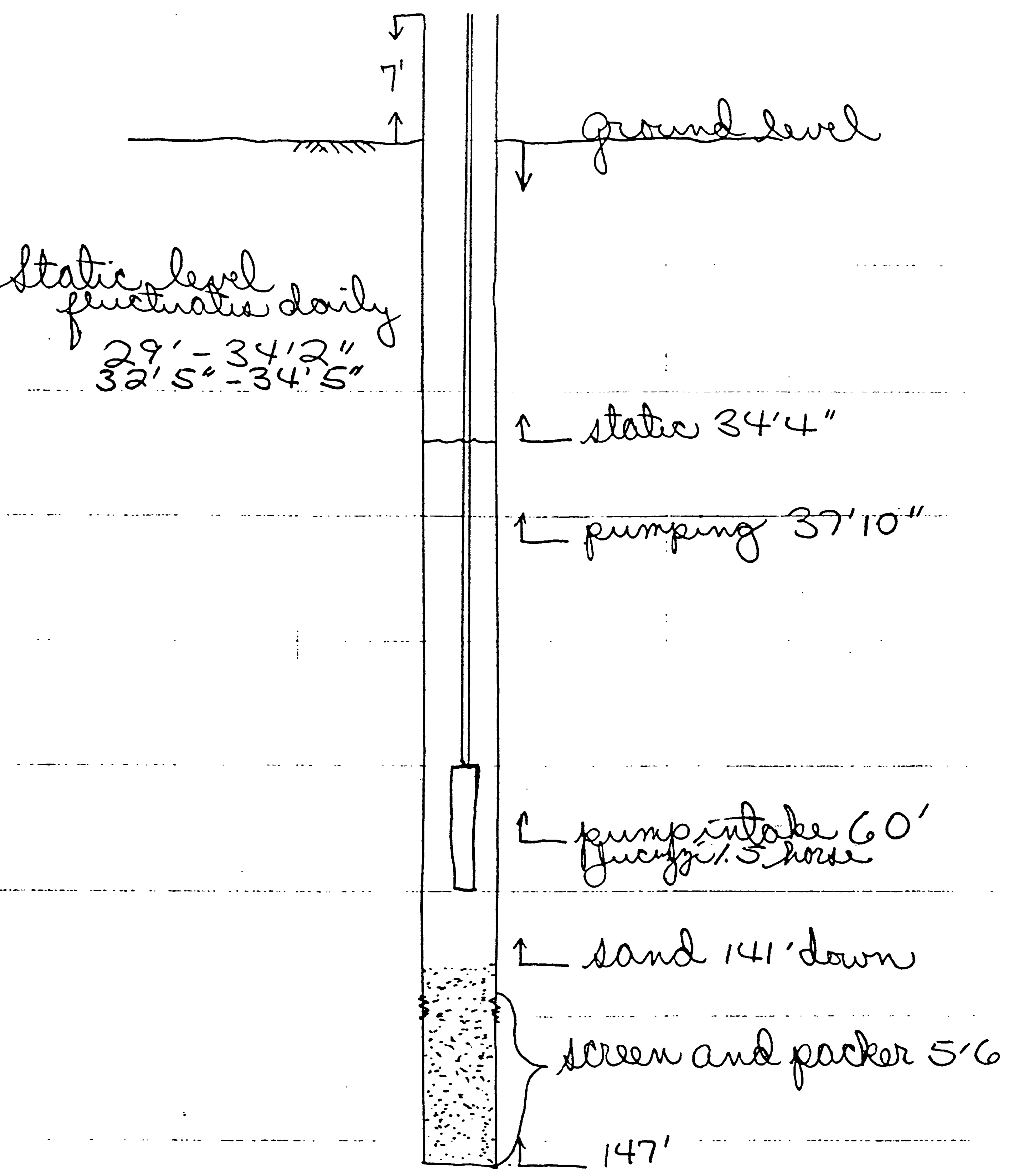

(49) 


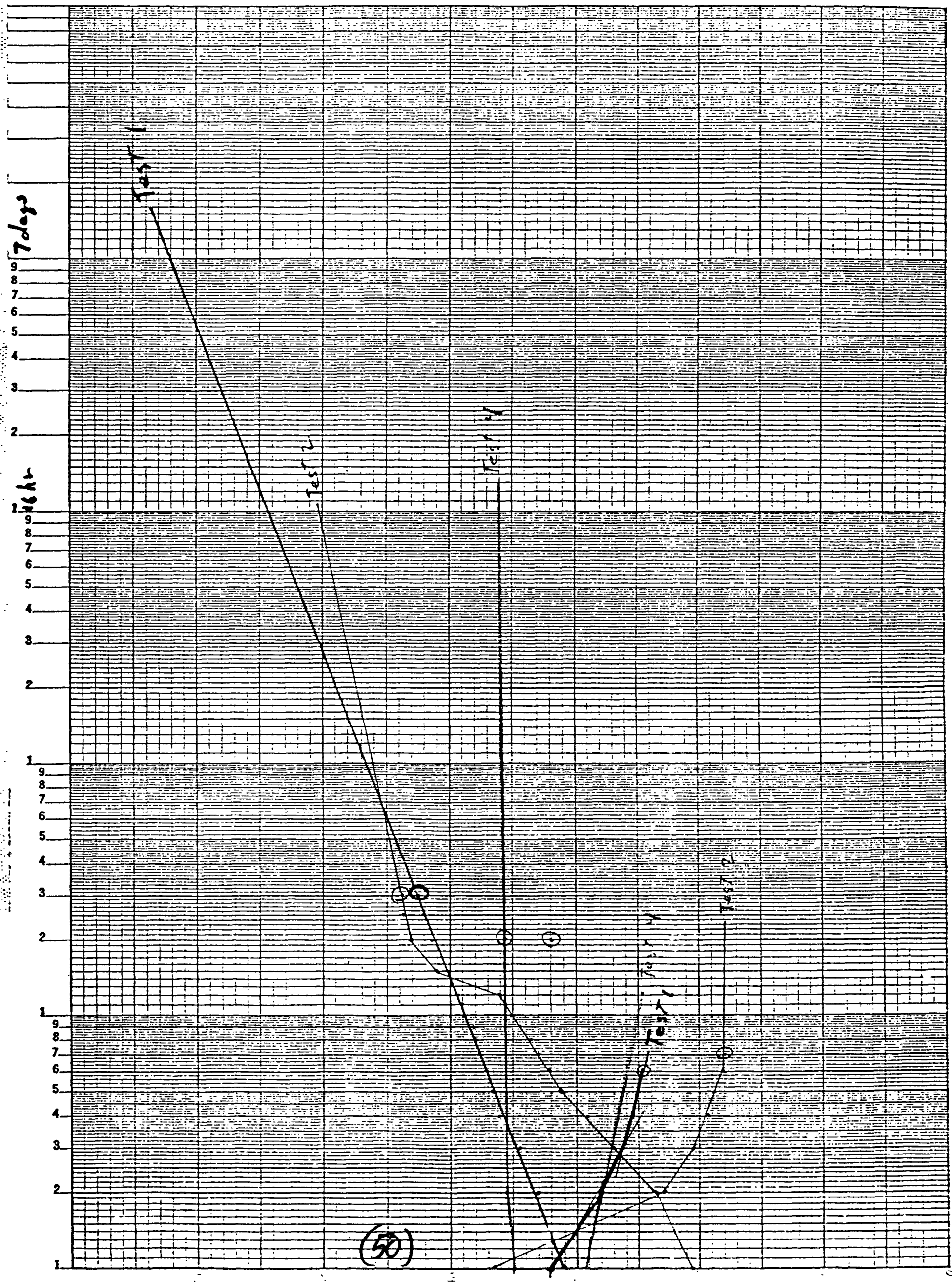


WELL LOG

U.S. PUBLIC HEALTH SERVICE, DIVISION OF INDIAN HEALTH

LOCATION

DATE COMPLETED

TOTAL DEPTH OF WELL GROUT
DATE STARTED DRILLER FT. CASING INSTALLED DIAMETER LENGTH ? SCREEN SIZE MFG. 8 FT.

HOLE DIAMETER CASING DIAMETER

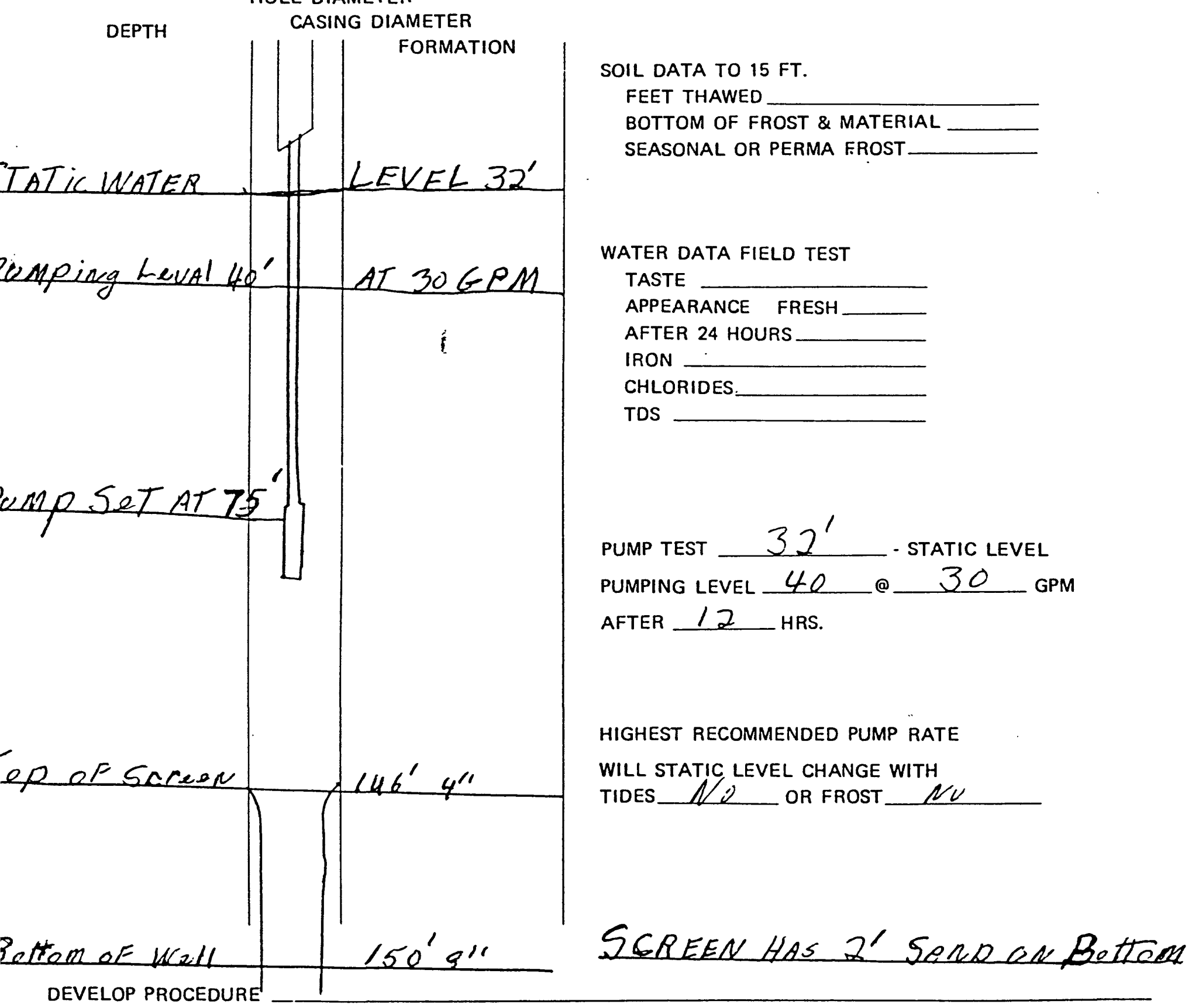

ESTIMATED MAN HOURS FOR DRILLING HOURS FOR TOTAL JOB CREW

(51) 


\section{CHEMICAL \& GEOLOGICAL LABORATORIES OF ALASKA, INC.}

TELEPHONE (907) 279-4014

P.O.BOX $4-1276$

ANCHORAGE, ALASKA 99509

4649 BUSINESS PARK BLVD.

ANALYTICAL REPORT

Water Analysis(Facility)__ Alaska Area Native Health Service

Date Collected: June 3, 1977 Time Collected:

By: Bill Hubbard

Source of Sample: treated water, Galena, Alaska.

Physical Observations, Remarks:

\begin{tabular}{|c|c|c|c|c|c|c|}
\hline[]$_{-}$ & $\mathrm{mg} / \mathrm{l}$ Aluminum & [] & mmhos Conductivity & 143 & $\mathrm{mg} / \mathrm{l}$ & Hardness as \\
\hline [] & $\mathrm{mg} / 1$ Arsenic & $x[x] \quad 7.0$ & units $\mathrm{pH}$ & $19 \quad 190$ & $\mathrm{mg} / \mathrm{l}$ & Alkalinity as \\
\hline [] & $\mathrm{mg} / \mathrm{l}$ Barium & {[]} & $\mathrm{mg} / 1$ Ammonia & {[]} & $\mathrm{mg} / \mathrm{l}$ & Acidity-T as \\
\hline[] & $\mathrm{mg} / 1$ Boron & [] & $\mathrm{mg} / 1$ Kjedahl & {[]$_{-}$} & $\mathrm{mg} / \mathrm{l}$ & Acidity Free \\
\hline[]$_{-}$ & $\mathrm{mg} / \mathrm{l}$ Cadmium & {[]} & $\mathrm{mg} / \mathrm{l}$ Organic & {[]} & $1100 \mathrm{ml}$ & Coliform-T \\
\hline W 41 & $\mathrm{mg} / \mathrm{l}$ Calcium & {[]$_{-}$} & $\mathrm{mg} / \mathrm{I}$ Nitrate(N) & {[]} & $/ 100 \mathrm{ml}$ & Coliform-F \\
\hline [] & $\mathrm{mg} / \mathrm{l}$ Copper & {[]} & $\mathrm{mg} / \mathrm{I}$ Nitrite(N) & {[]} & $1100 \mathrm{ml}$ & Strep-F \\
\hline [] & $\mathrm{mg} / \mathrm{l}$ Chromium-Total & {[]$_{-}$} & $\mathrm{mg} / \mathrm{l}$ Phosphorus & {[]$_{-}$} & units & Color \\
\hline[]$_{-}$ & mg/l Chromium-Tri & {[]} & $\mathrm{mg} / 1$ Phosphorus & {[]} & & \\
\hline [] & mg/l Chromium-Hex & {[]} & $\mathrm{mg} / \mathrm{l}$ Chloride & {[]$_{-}$} & & \\
\hline
\end{tabular}

$8 \mathbb{2} \quad 0.3 \mathrm{mg} / 1$ Iron-Total

[]

[

$\mathrm{mg} /$ I Iron-Dissolved []

$\mathrm{mg} / \mathrm{l}$ Cyanide

[]

$\mathrm{mg} / \mathrm{I}$ Lead

$\mathrm{mg} / \mathrm{l}$ Sulfate

89

[]

$\mathrm{mg} / \mathrm{l}$ Phenol

[]

$9.9 \mathrm{mg} / \mathrm{l}$ Magnesium

[]

$\mathrm{mg} / \mathrm{l}$ MBSA

[]

$\mathrm{mg} / \mathrm{l}$ Manganese

[]

$\mathrm{mg} / \mathrm{I} B O D$

[]

$\mathrm{mg} / \mathrm{l}$ Mercury

[]

$\mathrm{mg} / \mathrm{l} C O D$

$\cdot[]$

$\mathrm{mg} / \mathrm{l}$ Nickel

$\mathrm{mg} / 1$ Potassium

[]

$\mathrm{mg} / \mathrm{l}$ Selenium

[]

$\mathrm{mg} / \mathrm{l}$ Sodium

[

$\mathrm{mg} / \mathrm{l}$ silver

[]

$\mathrm{mg} / \mathrm{l}$ Zinc

$\mathrm{mg} / \mathrm{l}$ TO Solids

[]

$\mathrm{mg} / \mathrm{l}$ TV Solids

[]

$\mathrm{mg} / \mathrm{l}$ Suspended

Solids

[]

$\mathrm{mg} / \mathrm{l}$ SV Solids

Transported by:

Received by:

Transported by:

Received by:

FOR LAB USE ONLY

Lab\#_ 6015 Rec'd by: Se

Date sample rec'd: June 9, 1977

Date analys is completed: $6-13-77$

Date results reported: $6-14-77$

signed:Ahcliceng Leex

Date: June 14, 1977 
CORRECTED. CoPY

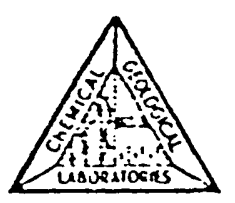

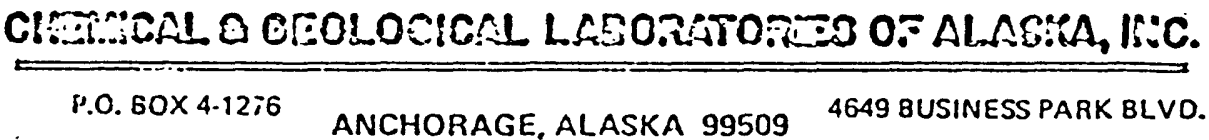

TELEPHO:V (IDO) 273.4

\section{ANALYTICAL REPORT}

Water Analysis(Facility). Alaska Area Native Health Service

Date Collected: $3-31-78$

Time Collected:

By: P. Besaw

Source of Sample:_ Salena, Alaska Well No. 2

Phjsical Ooservations, Remarks: Treatment: None Preservative: None

\begin{tabular}{|c|c|c|c|c|}
\hline[]$\equiv=5$ & mgil Aluminum & [] 320 & mmhos Conductivity & $\mathrm{mg} / \mathrm{l}$ Hardness as \\
\hline$\leq 0.05$ & mgil Arsenic & 6.5 & units $\mathrm{PH}$ & Pinity a: \\
\hline & $\mathrm{mg} / 1$ Barium & {[]} & \multirow{4}{*}{$\begin{array}{l}\text { Ammonia } \\
\text { Nitrogen-N } \\
\text { Kjedahl } \\
\text { Nitrogen-N } \\
\text { Organic } \\
\text { Nitrogen-N } \\
\text { Nitrate(N) }\end{array}$} & ity-T as \\
\hline & mig/l Eoron & {[]} & & ity Free \\
\hline & $m g / 1$ Câdmium & & & $1100 \mathrm{ml} \mathrm{Co}$ \\
\hline 34 & m!'? Caicium & & & $/ 100 \mathrm{ml}$ Coliform-F \\
\hline & ma/l Copper & & mg/l Nitrite(N) & $1100 \mathrm{ml}$ Strep-F \\
\hline & mgi! Cinromium-Total & {[]} & \multirow{3}{*}{$\begin{array}{l}\text { Phosphorus } \\
\text { (Ortho)-p } \\
\text { Phosphorus } \\
\text { (Total)-P } \\
\text { Chloride }\end{array}$} & units Color \\
\hline & mg/1 Chromium-Tri & {[]} & & {[]} \\
\hline & $\mathrm{mg} / 1$ Chromium-ilex & {[]} & & {[]} \\
\hline $4^{2}$ & mg/l Iron-Total & [] & $\mathrm{mg} / 1$ Fluoride & \\
\hline & $m g / 1$ Ircn-Dissolyed & {[]} & mg/l Cyanide & \multirow{3}{*}{$\begin{array}{l}\text { Transported by: } \\
\text { Received by: } \\
\text { Transported by: } \\
\text { Received by: }\end{array}$} \\
\hline & $\mathrm{mg} / 1$ Lead & {[]$\leq 1$} & $m g / l$ Sulfate & \\
\hline 12 & $\mathrm{mg} / 1$ Magnesium & & mg/l Phero: & \\
\hline 1.3 & mg!l Manganese & {[]} & $\mathrm{ma} / 1 \mathrm{MBSA}$ & \multirow{6}{*}{ 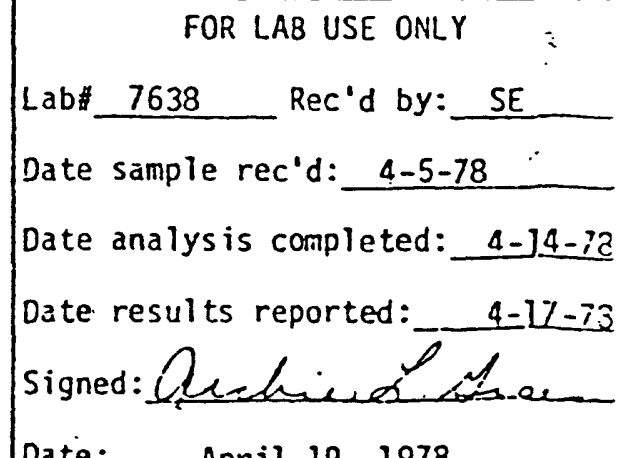 } \\
\hline & mgil Mercury & L & $\mathrm{mg} / 1800$ & \\
\hline & mg/l Nickel & {[]} & $m g / 1 \operatorname{coD}$ & \\
\hline & mg/l Potassium & [] 204 & $\mathrm{mg} / \mathrm{l}$ TD Solids & \\
\hline & $\mathrm{mg} / \mathrm{l}$ Selenium & [] & $\mathrm{mg} / 1$ TV Solids & \\
\hline 2.4 & $\mathrm{mg} / 1$ Sodium & {[]} & $\mathrm{mg} / \mathrm{l}$ Susfended & \\
\hline & mg/l Silver & & mg!l sV solids & April 19. 1978 \\
\hline & ma/l ?iric & & TI Turbidity & \\
\hline
\end{tabular}




\section{APPENDIX 5}

Summary of USGS Ground Water Site Inventory Data

and selected well drillers' logs and water-quality data for Galena

from U.S. Geological Survey village files 


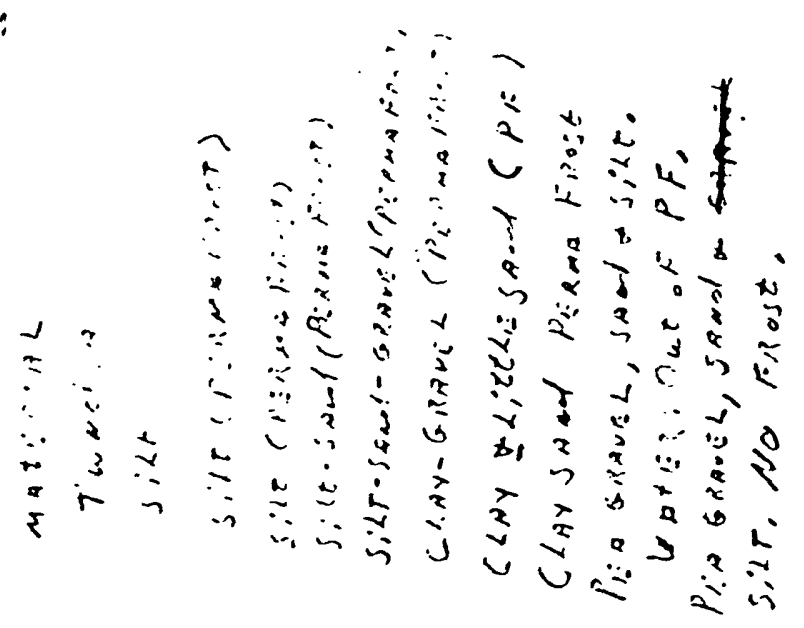

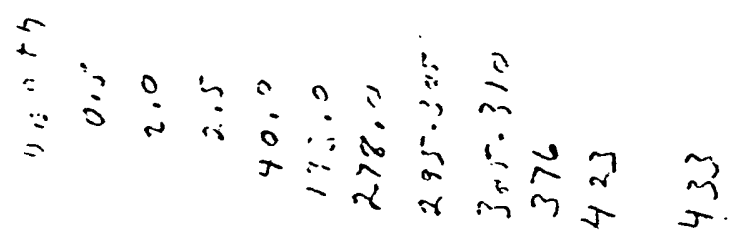

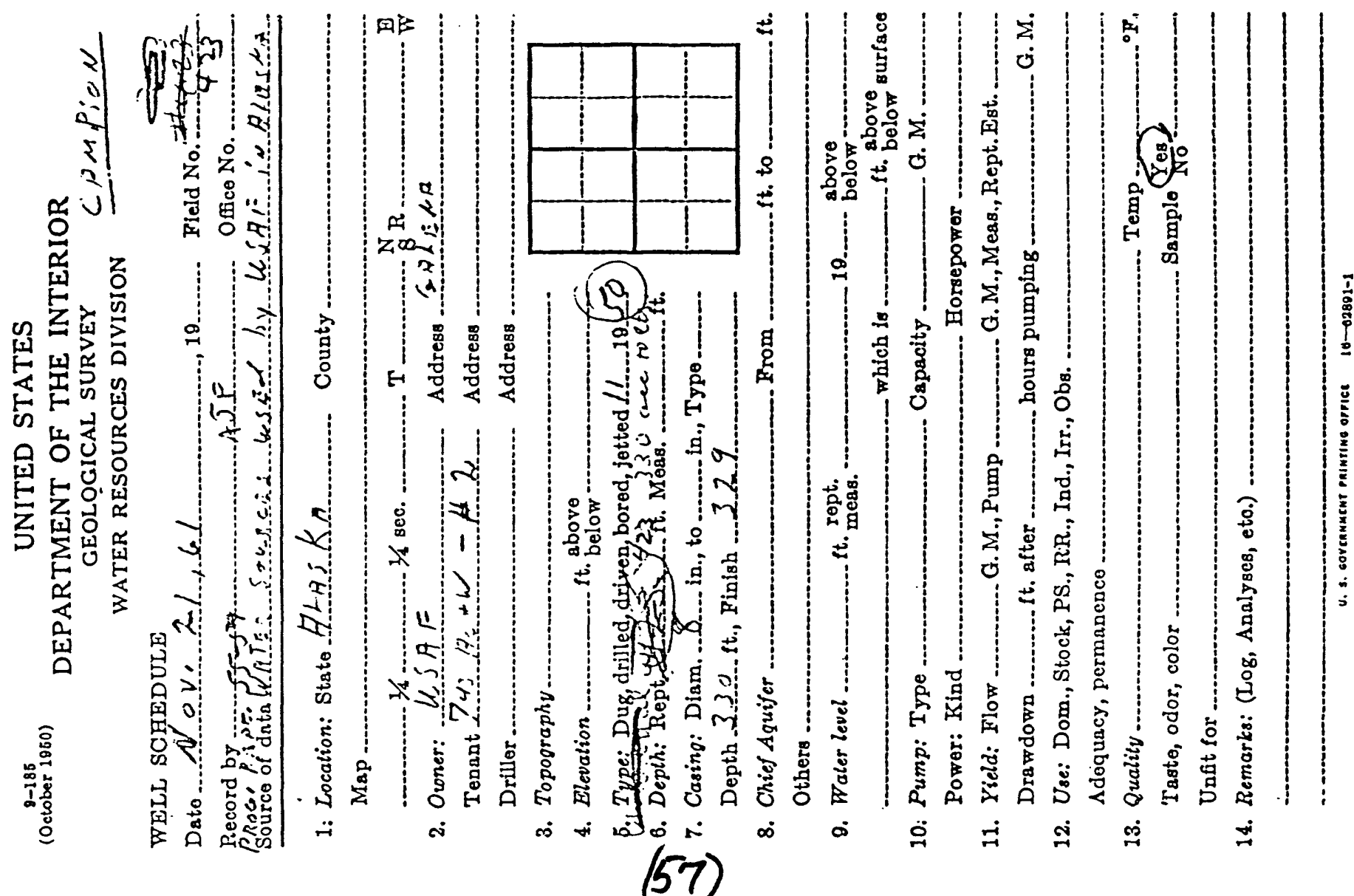



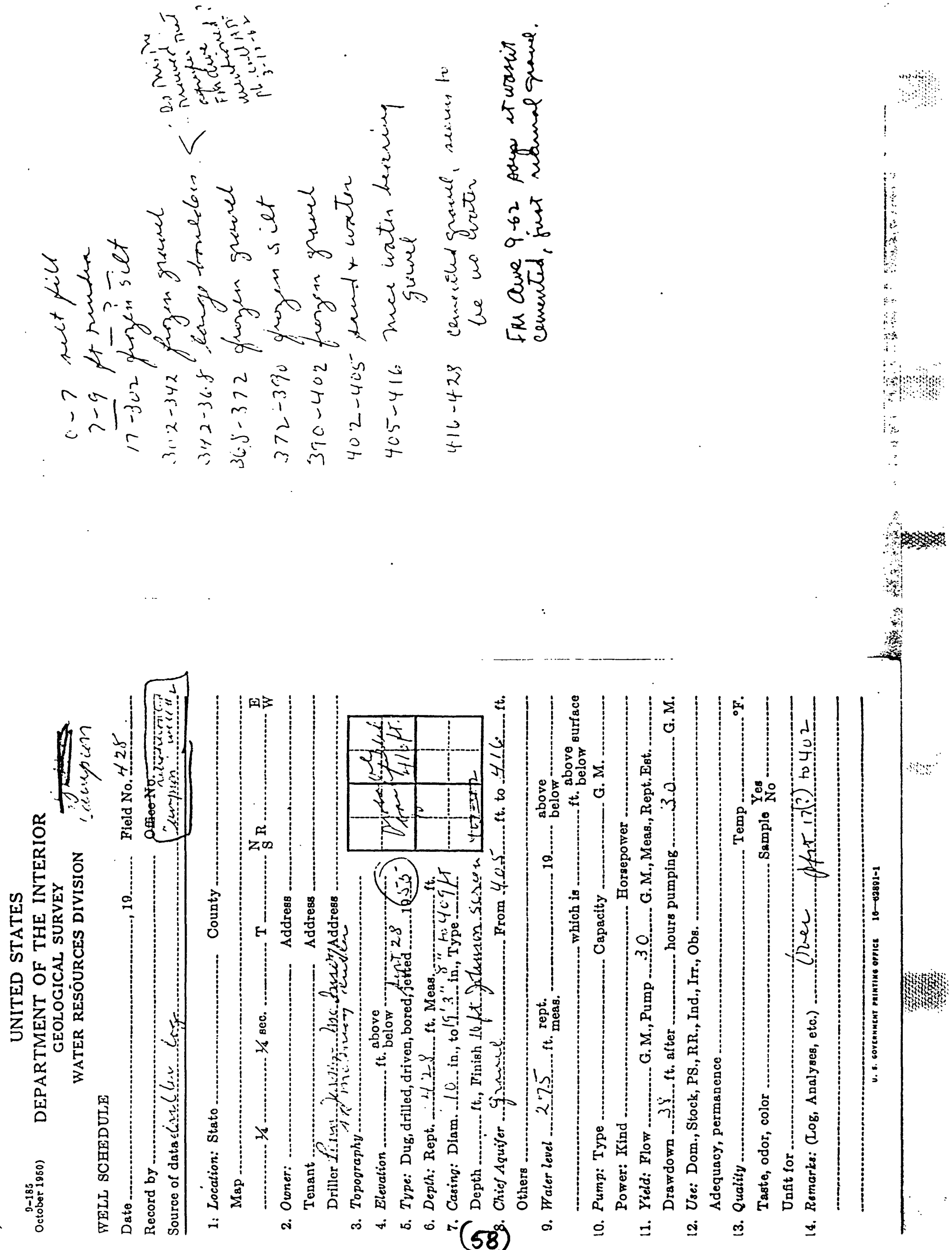


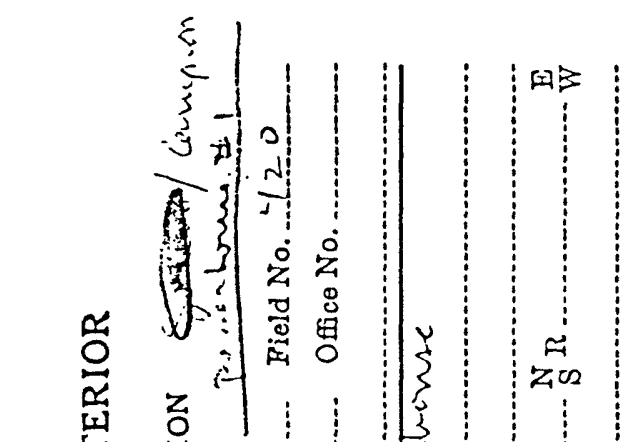

艺空

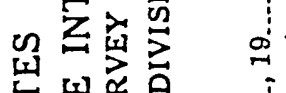

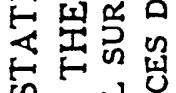

的牙然

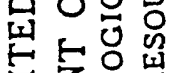

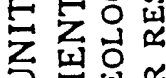

$\sum_{0}$

近 3

高

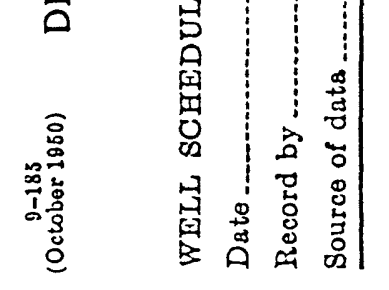

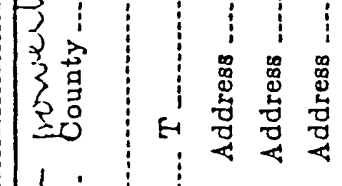

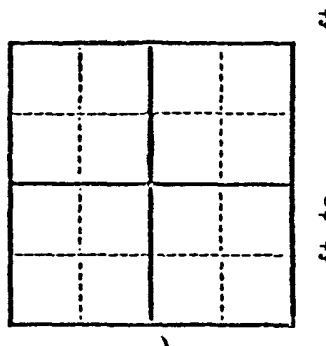

然至

莕离离

0
0
0

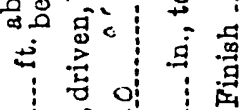

$+$

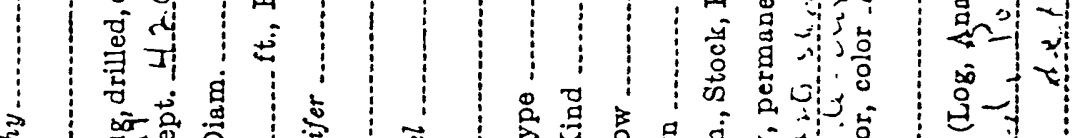

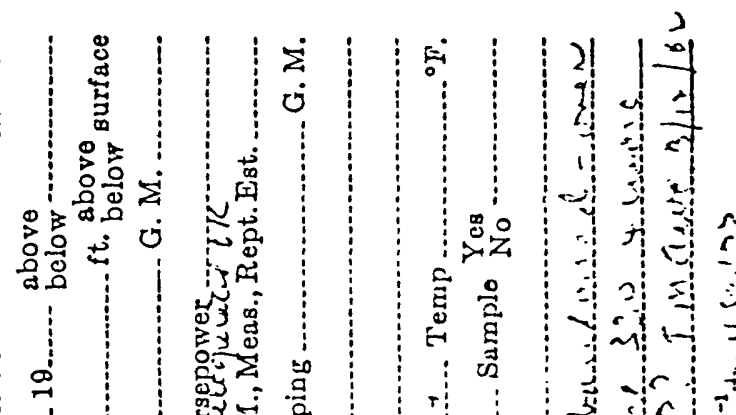

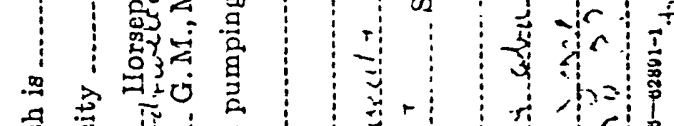

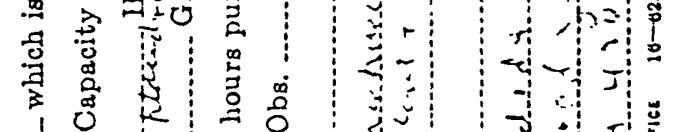

倠

岁

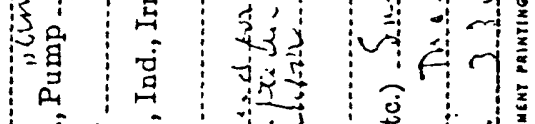

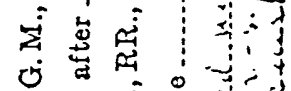

:

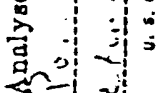

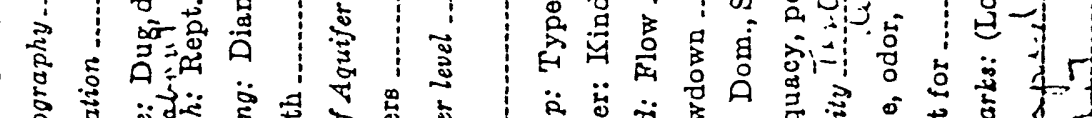

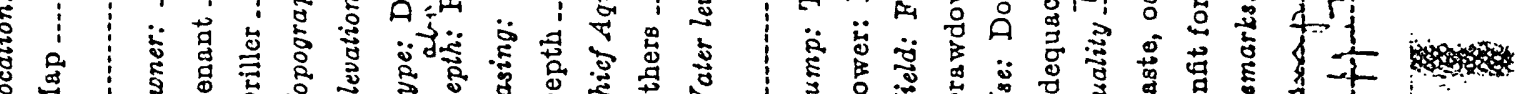

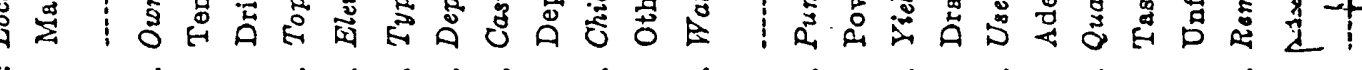

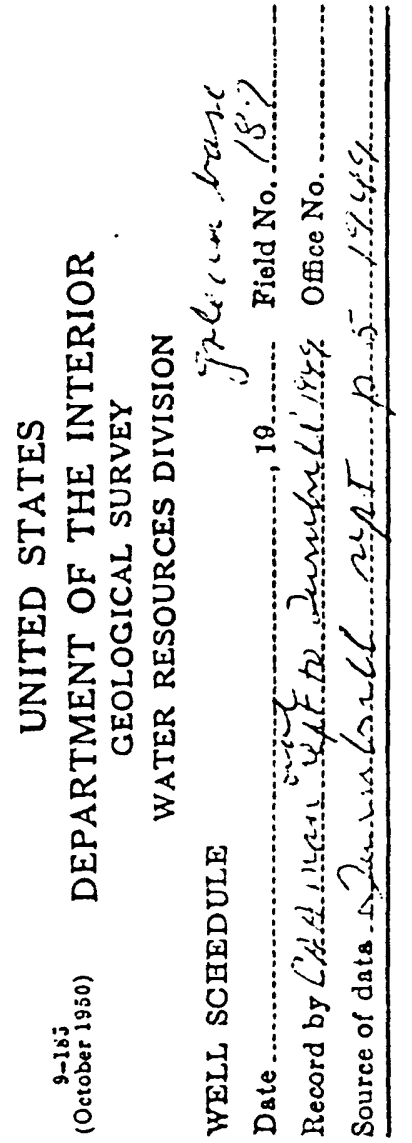

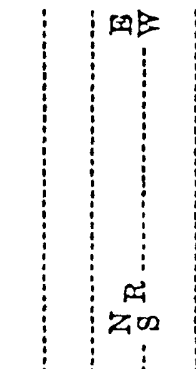

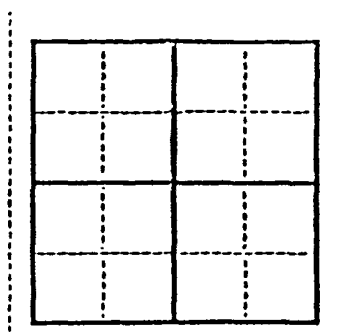

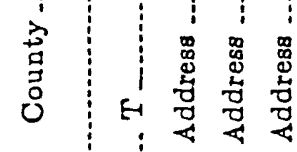

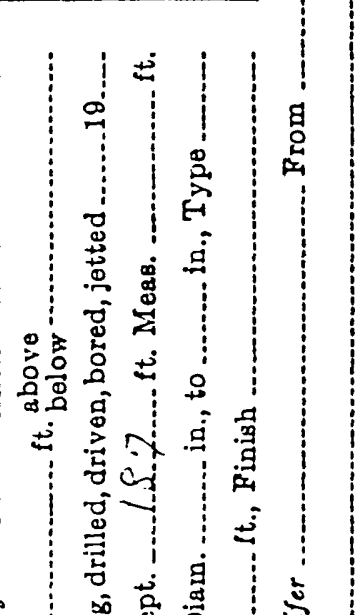

莕恋

-

离

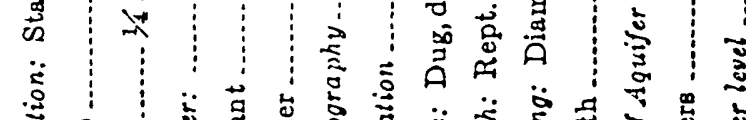

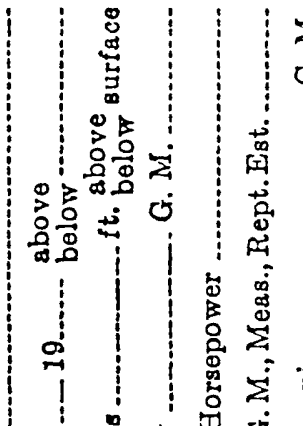

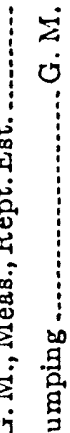

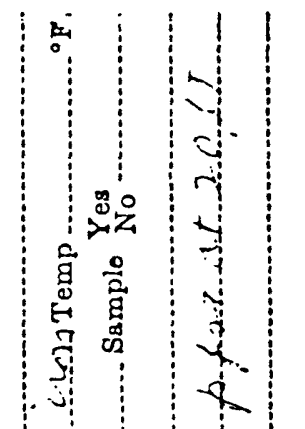

$\therefore$ 今 0 出

离

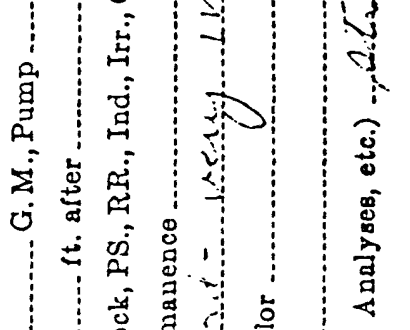

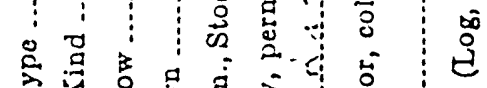

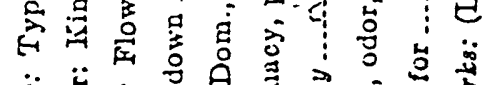

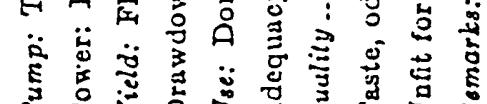

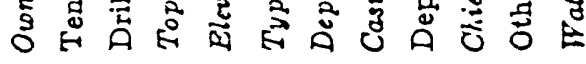

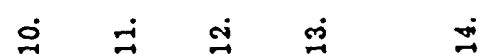




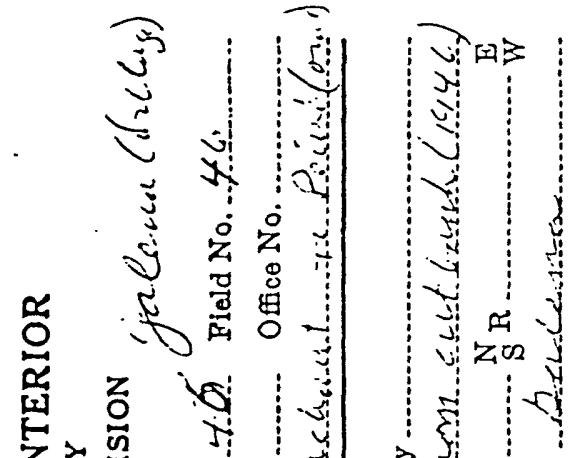

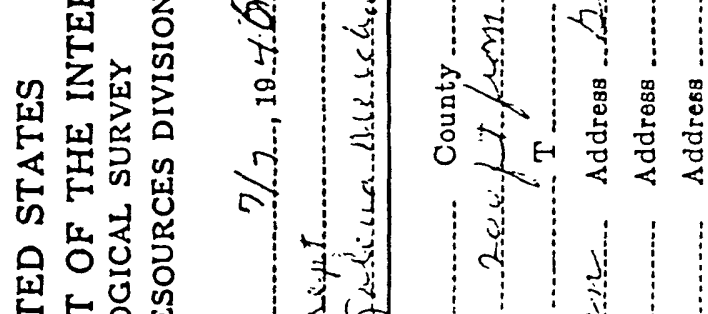

政

35

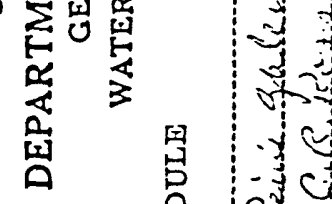

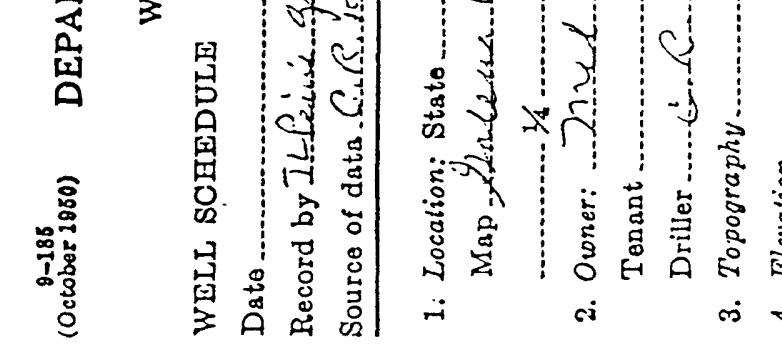

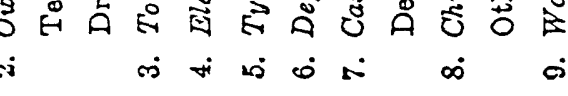

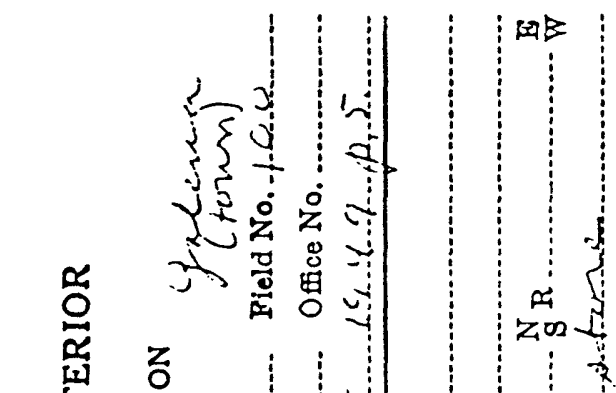

点变

四吕

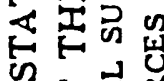

约它苔

봅엉

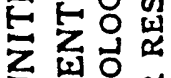

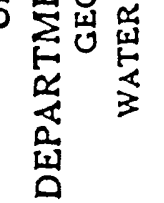

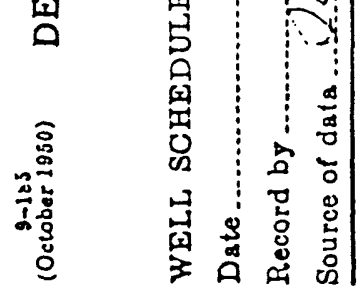

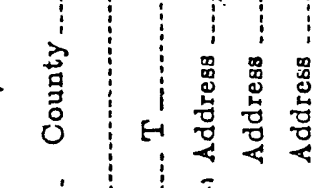

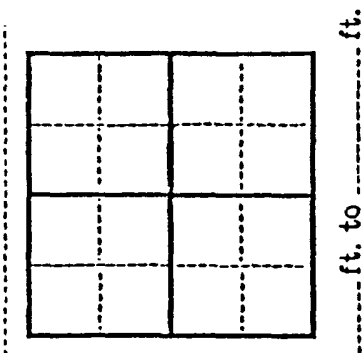

3
$j$
0
0
0

牙

$\lambda$

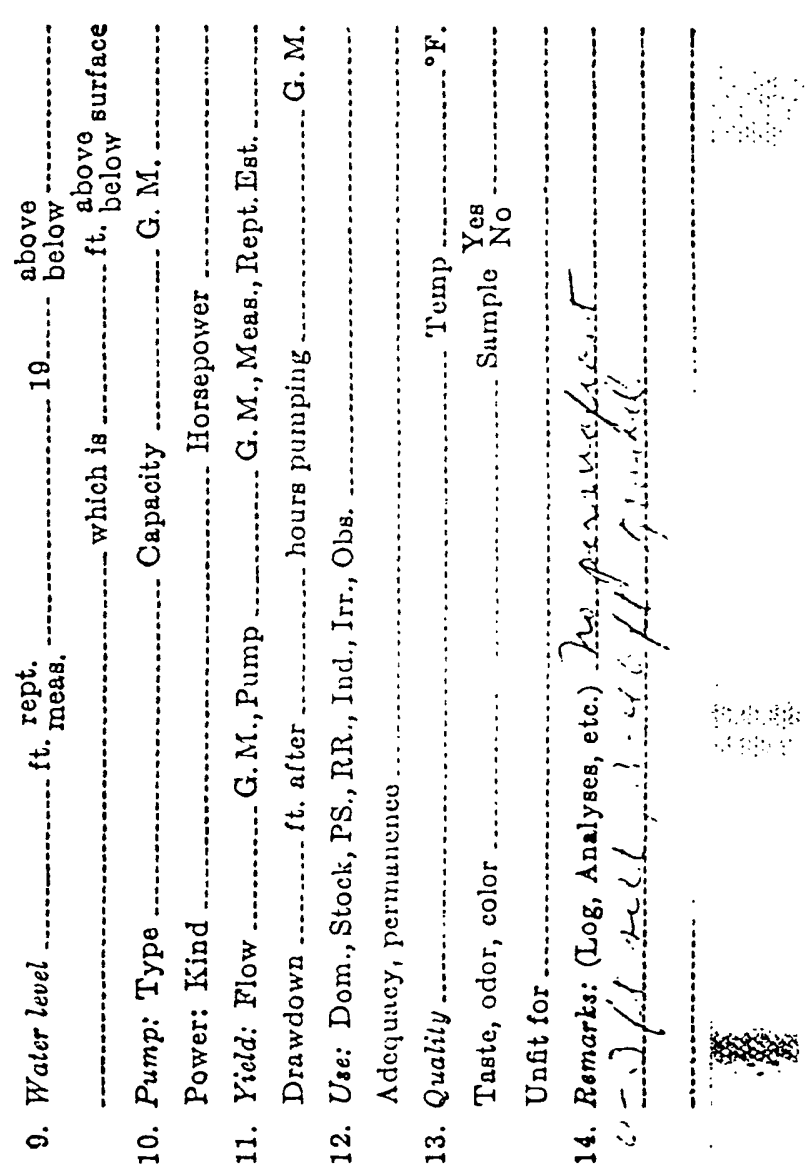

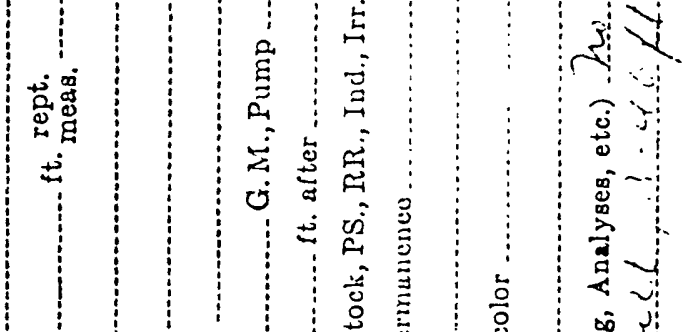

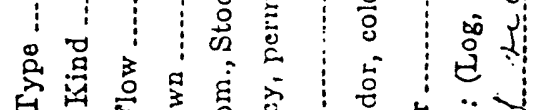

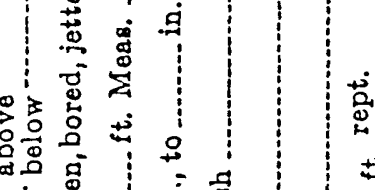
至离要

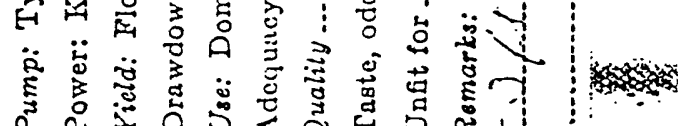

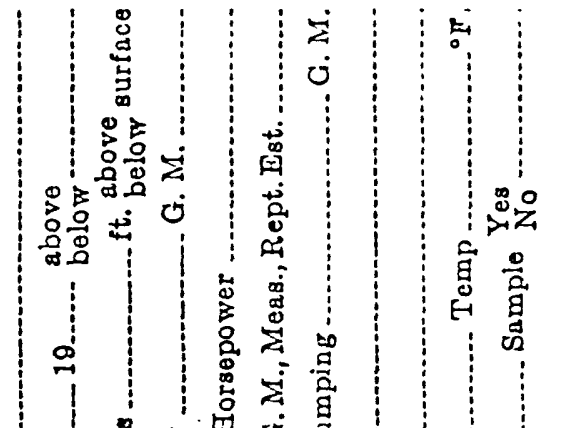

$\stackrel{0}{\circ} 0$

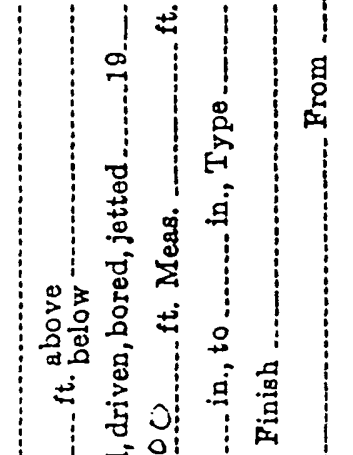

莒品

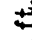



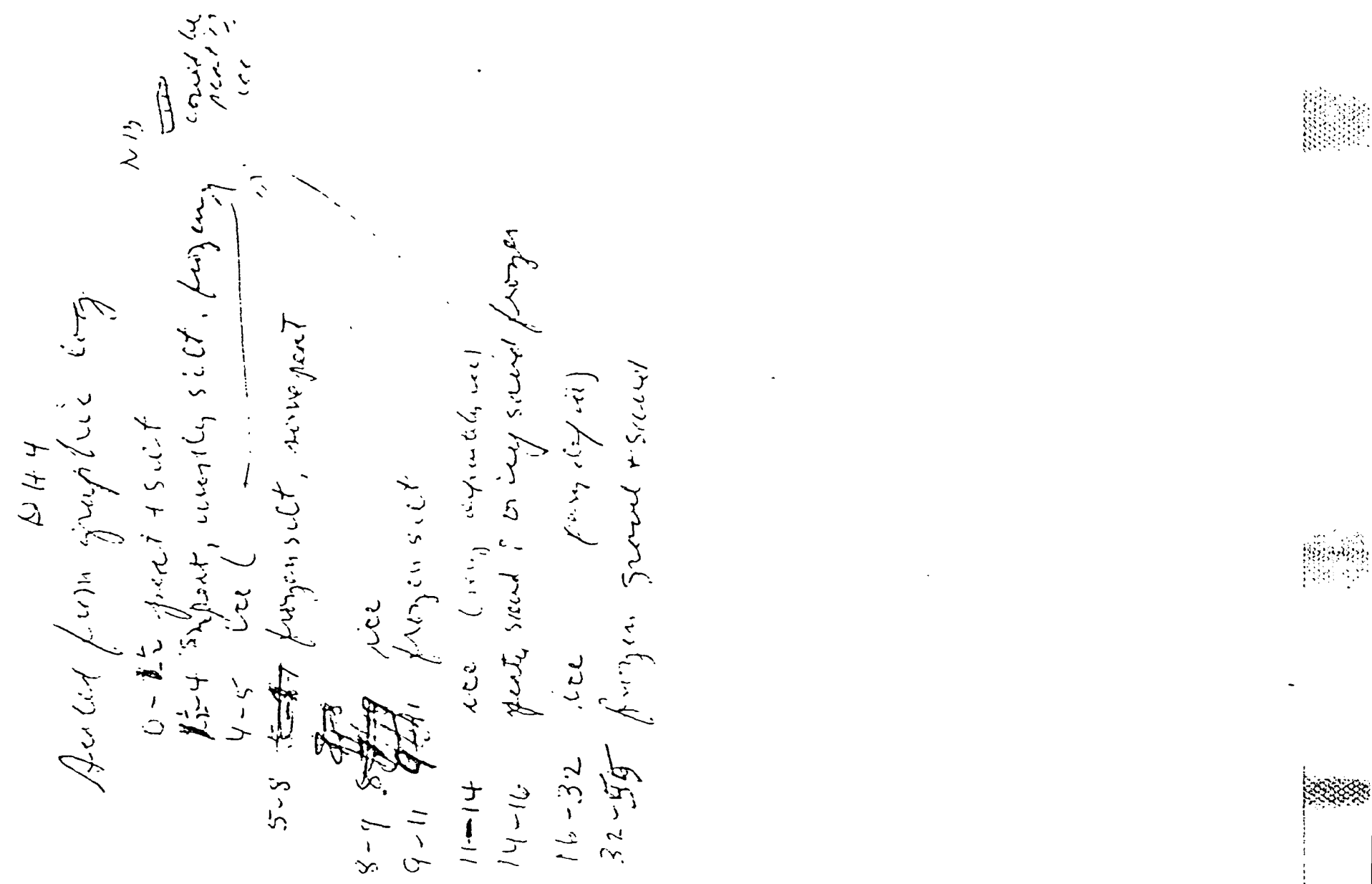

os?
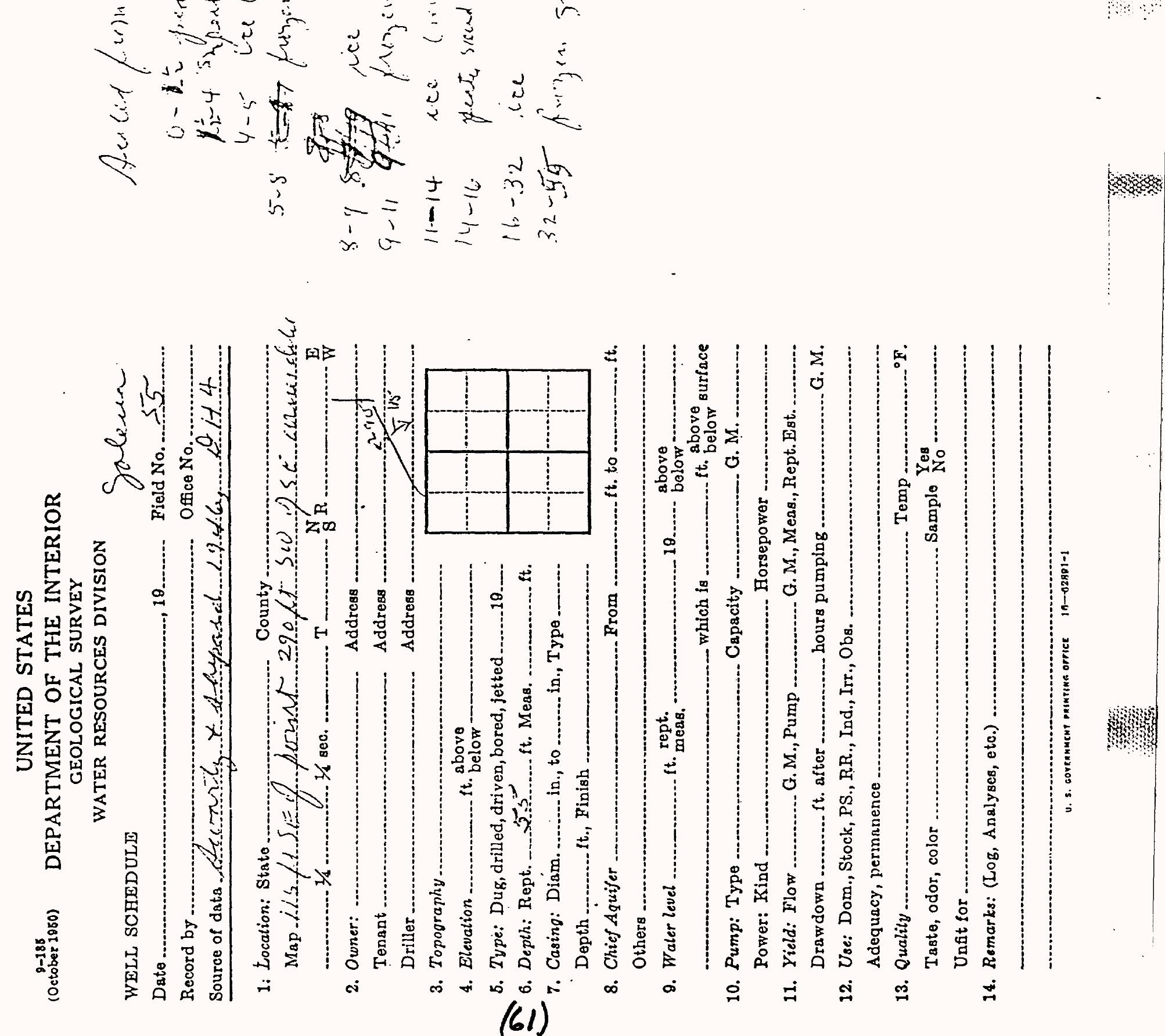

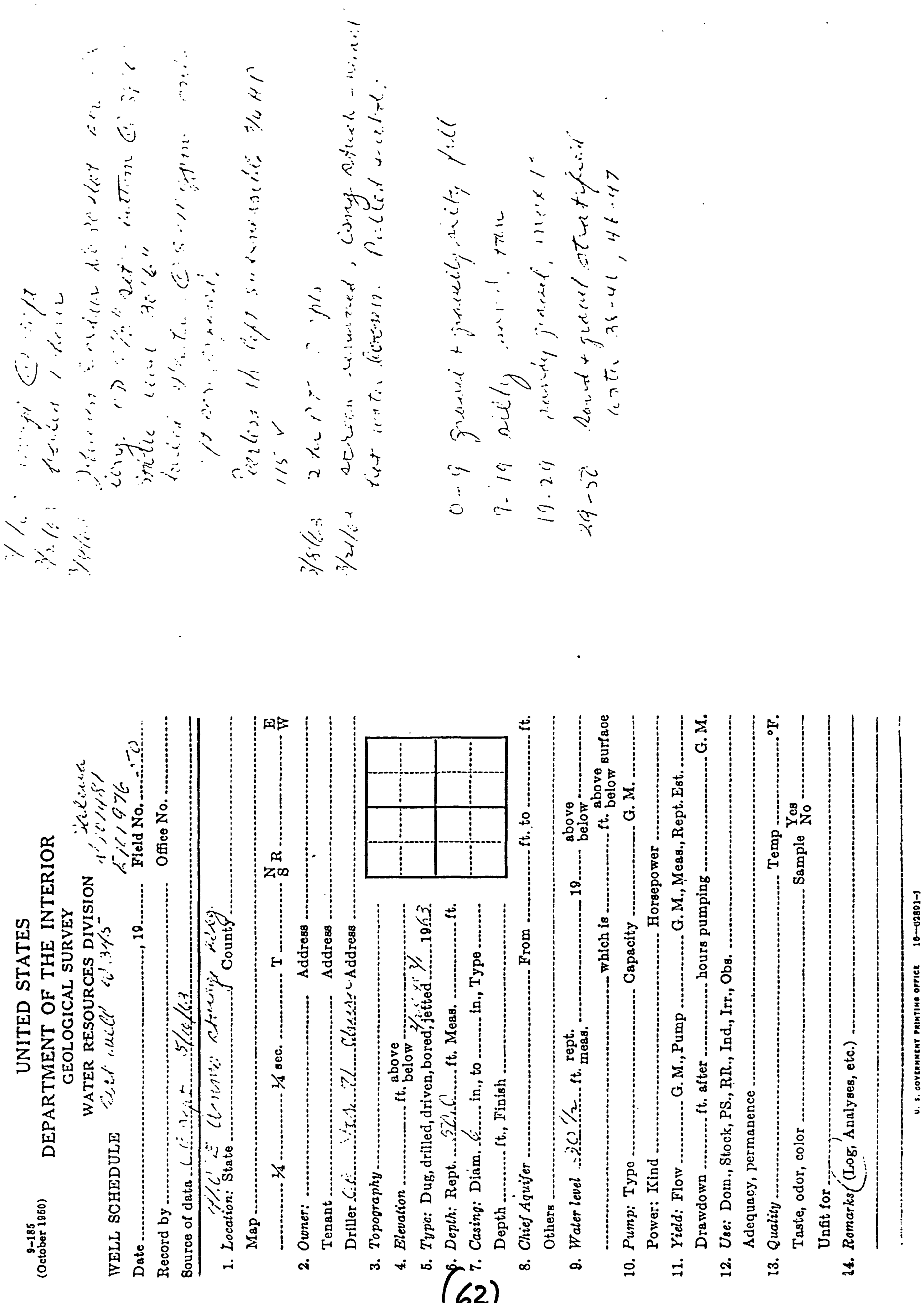


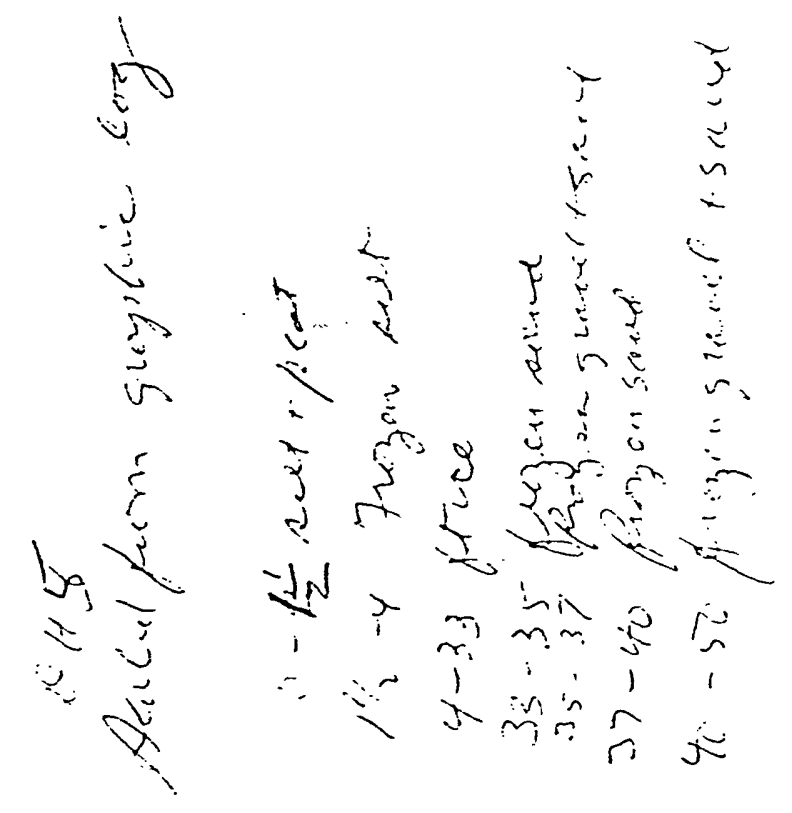

皮

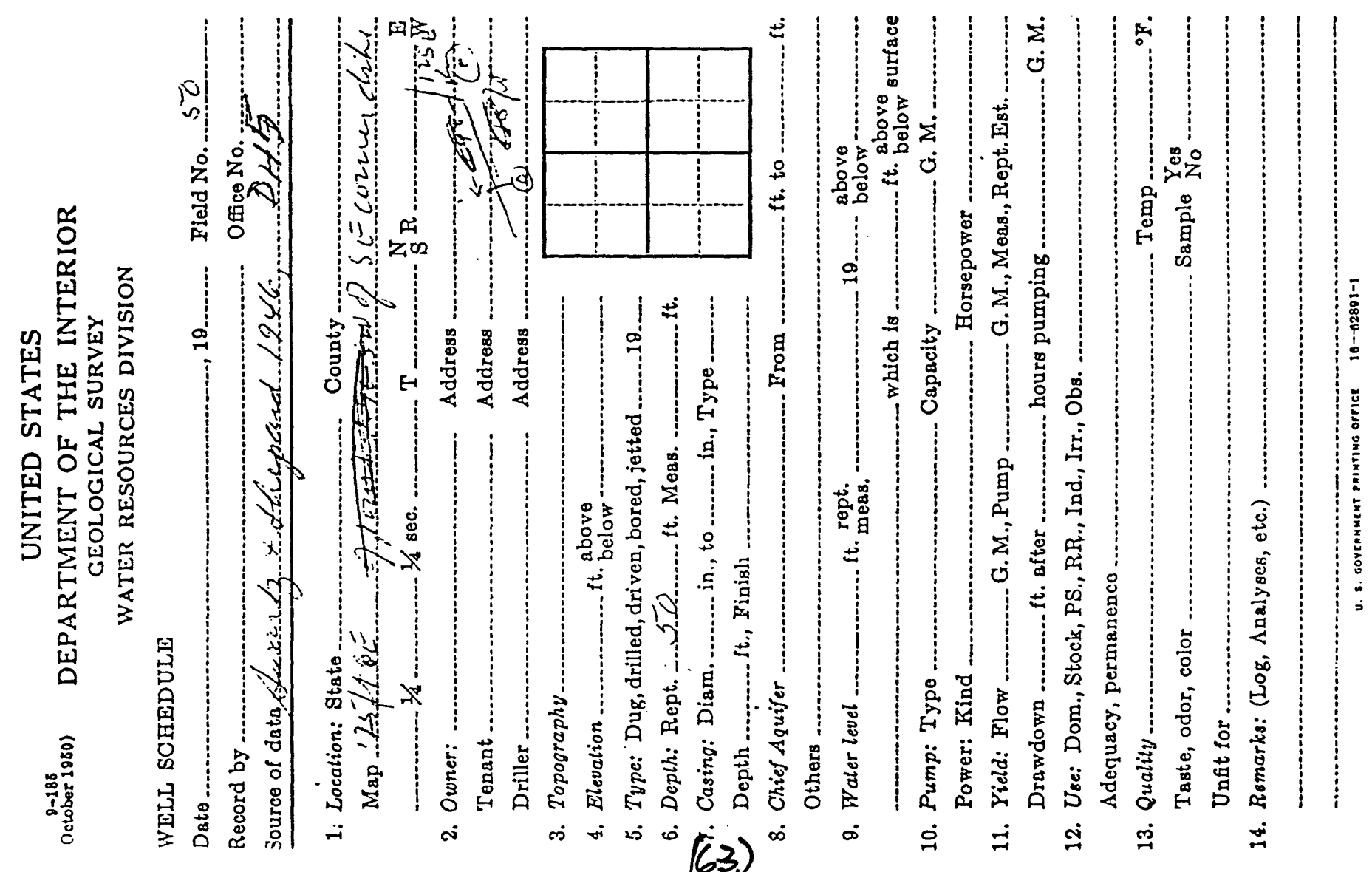




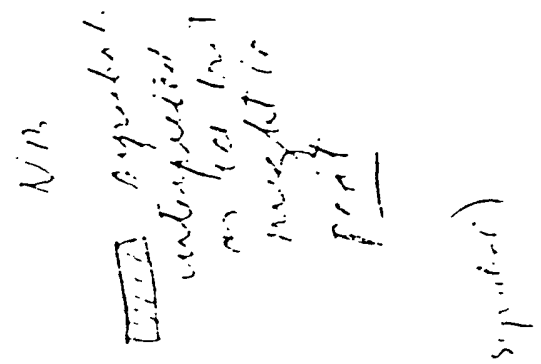

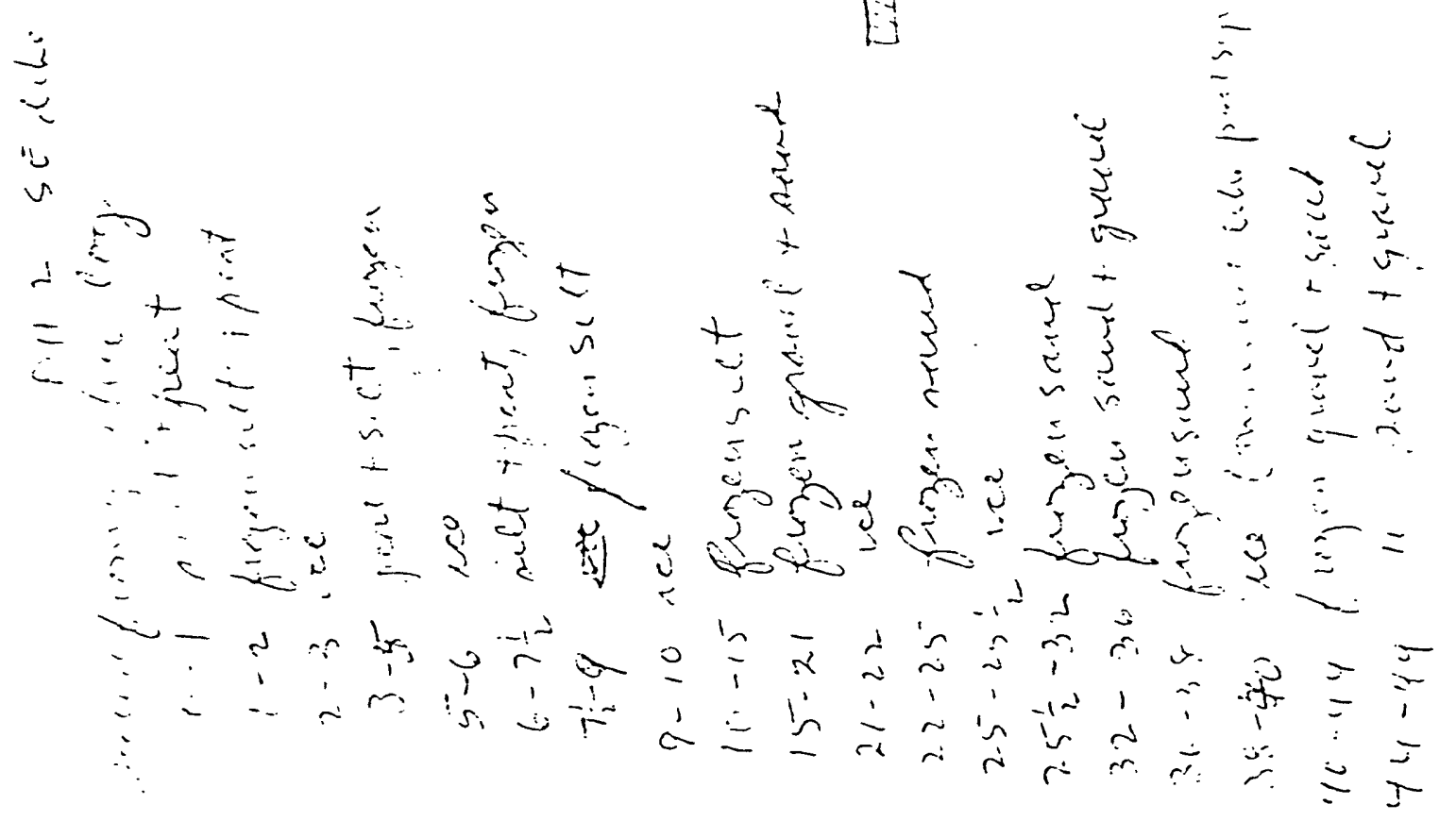

4

家

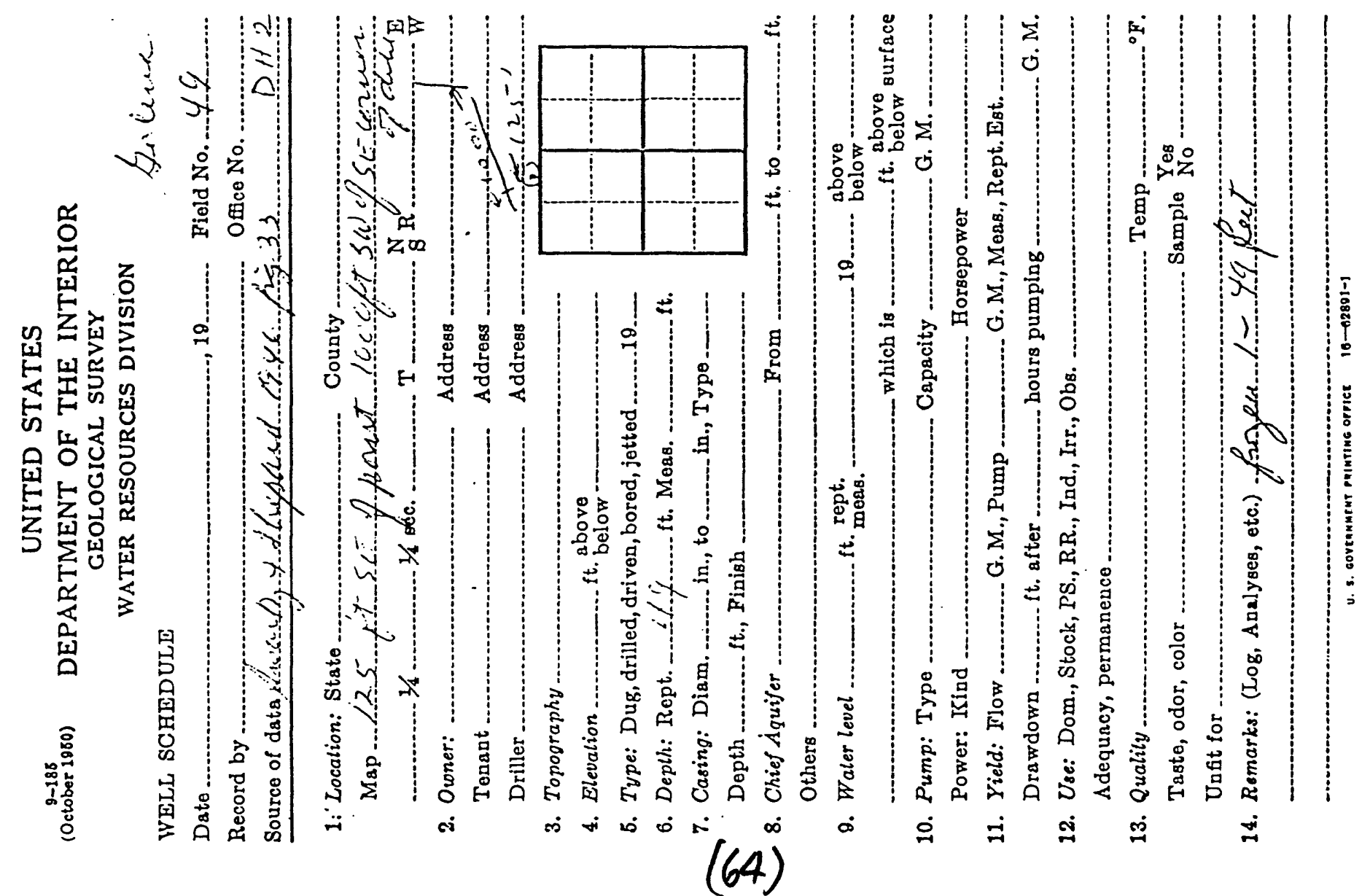




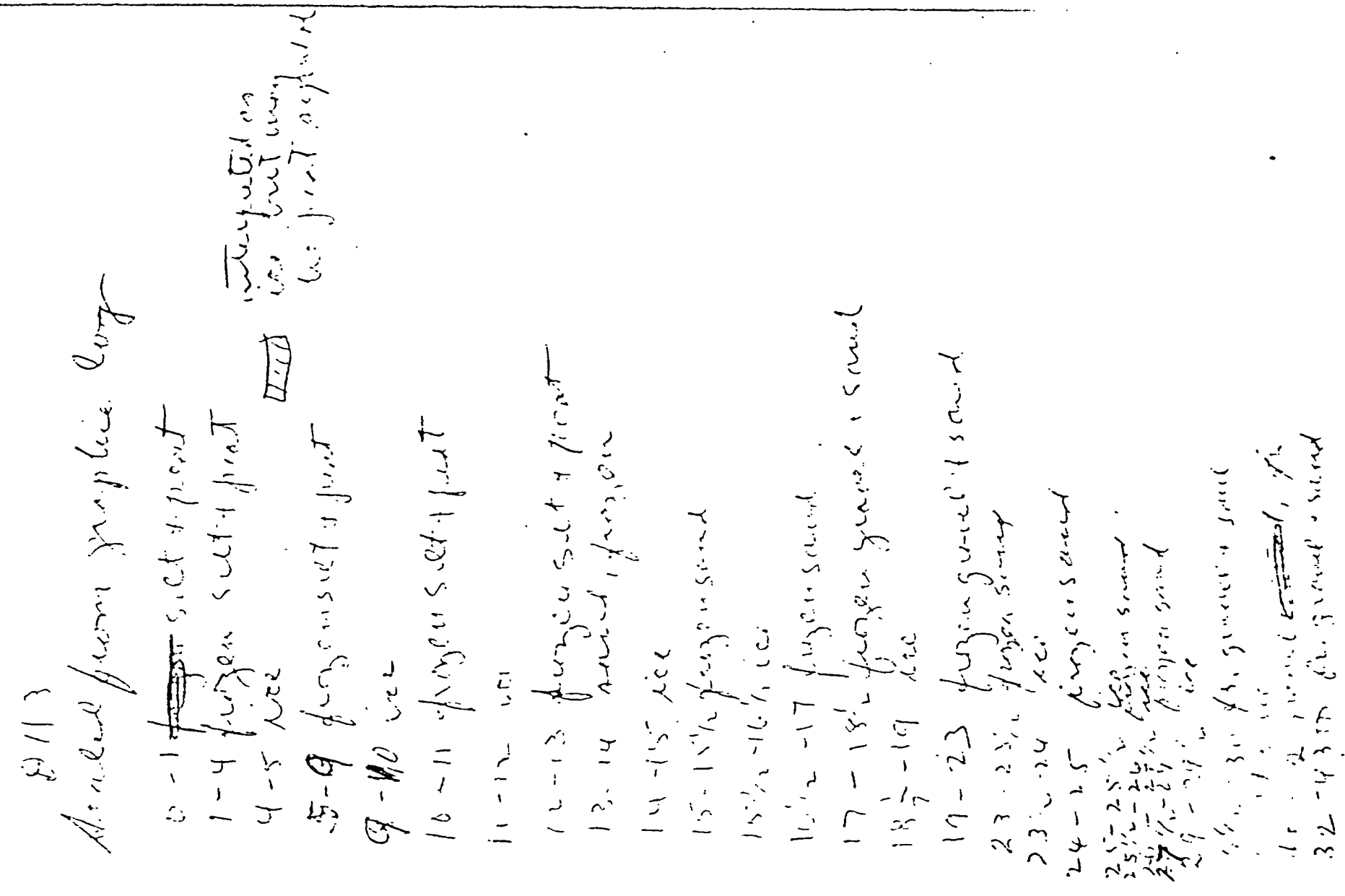

告

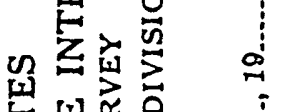

出范

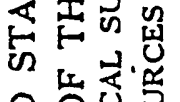

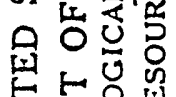

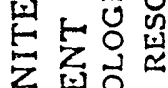

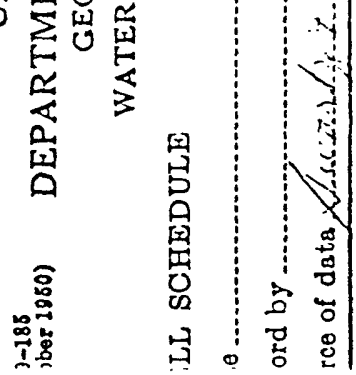

然

方

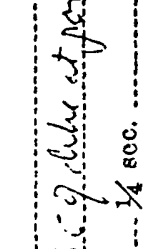

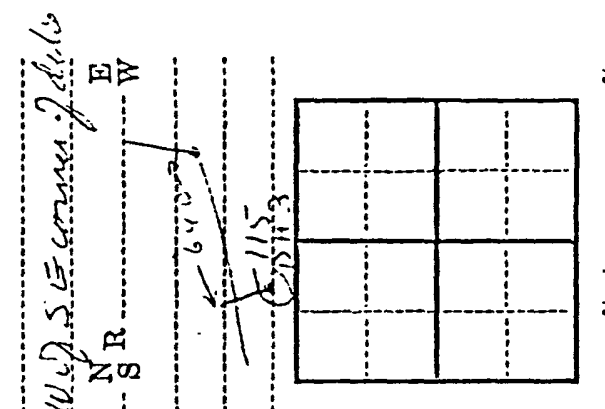
है से

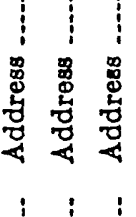

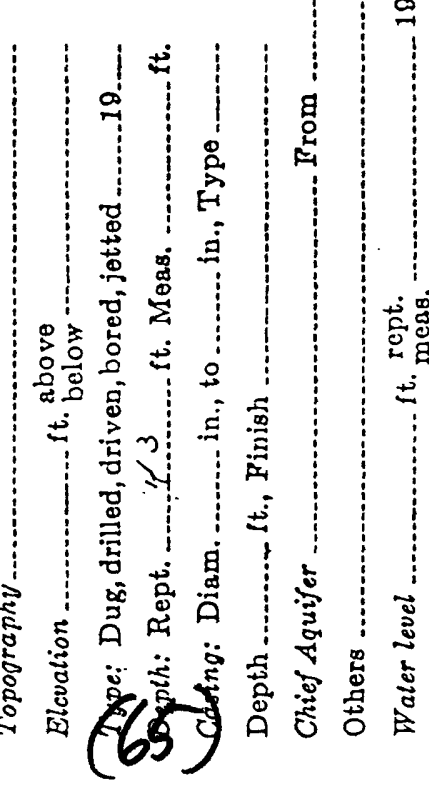

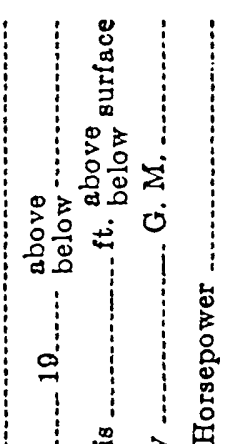

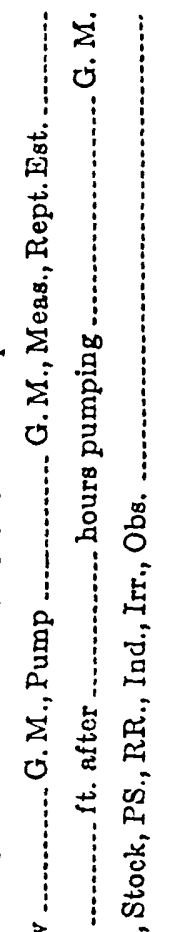

品

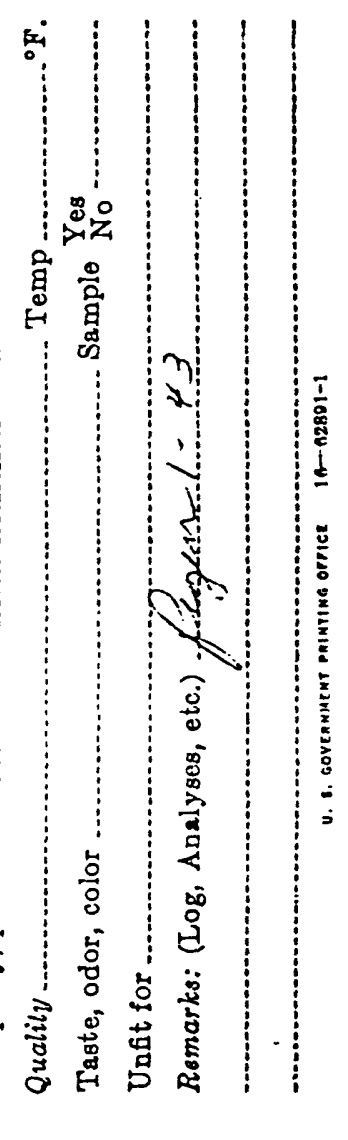




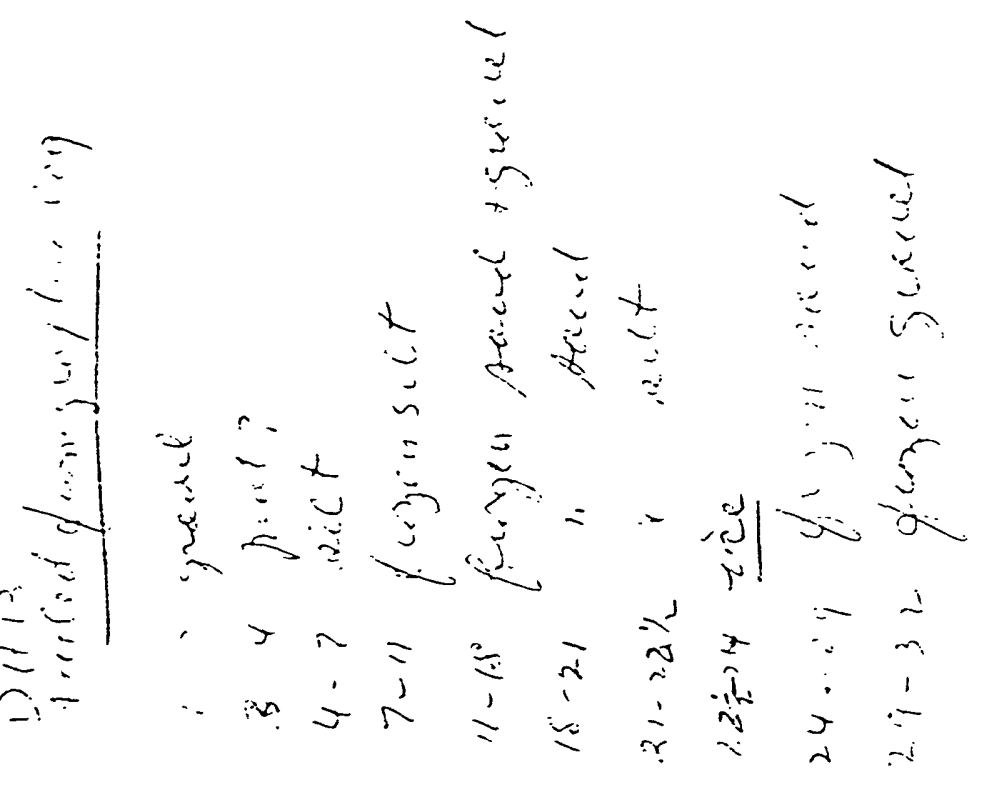

s
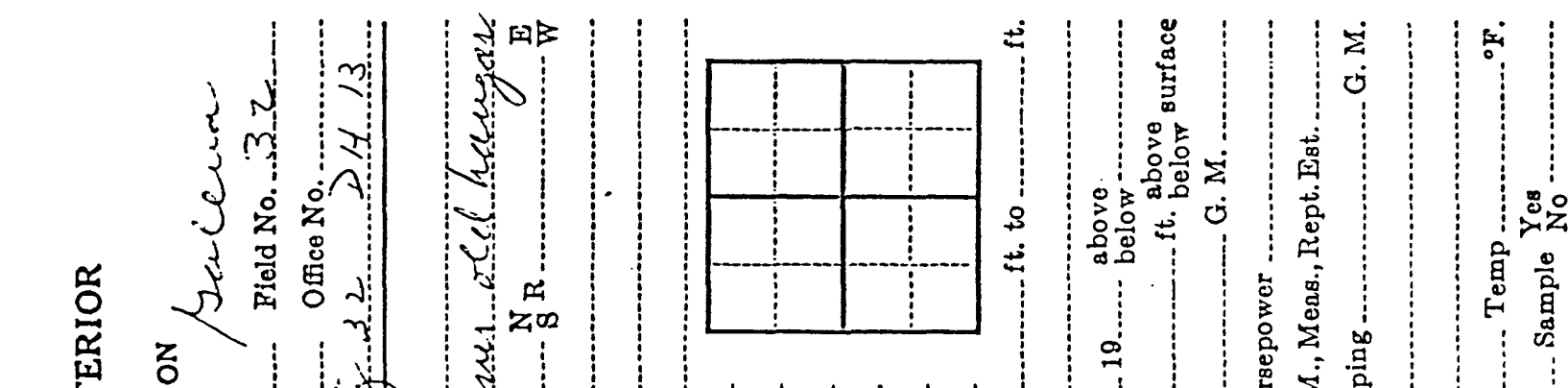

.

离

4

年

娄总

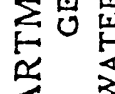

蜜

宷

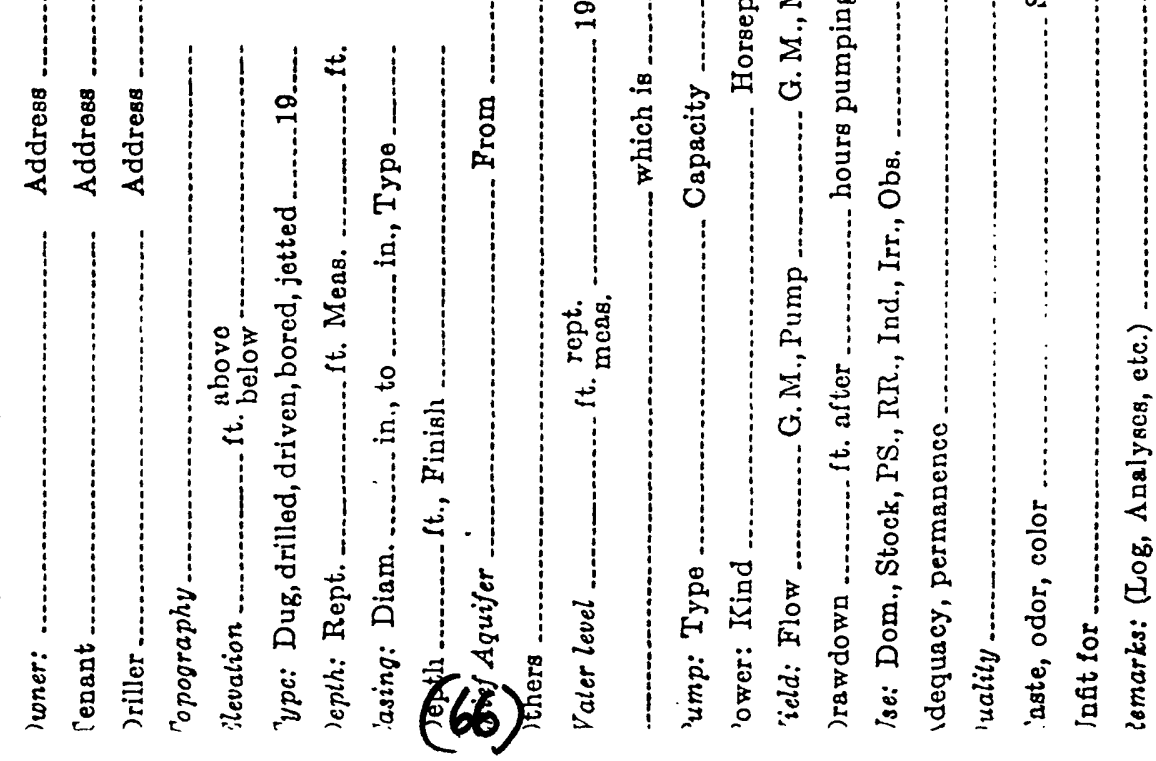




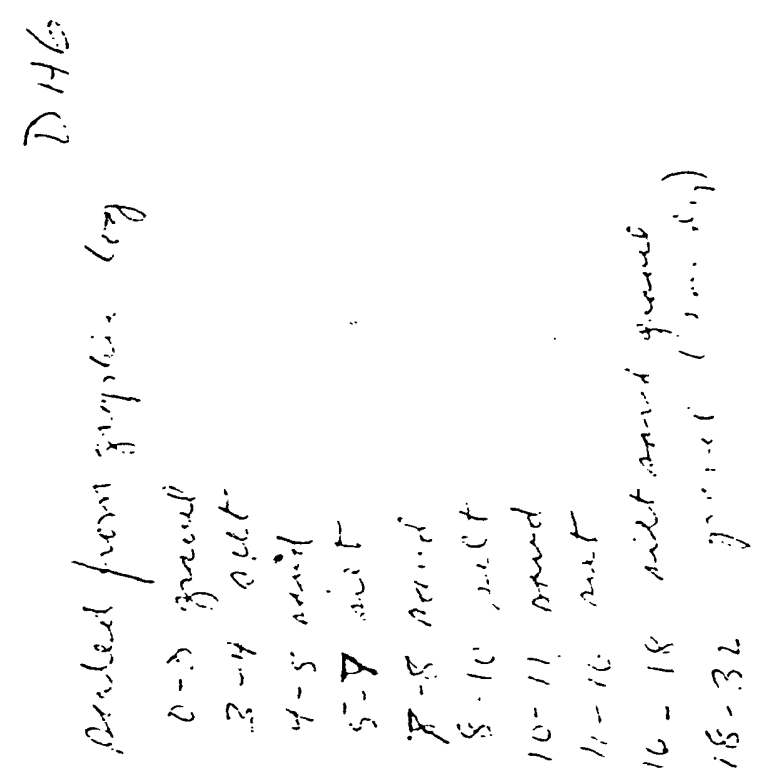

\section{4}

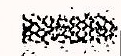

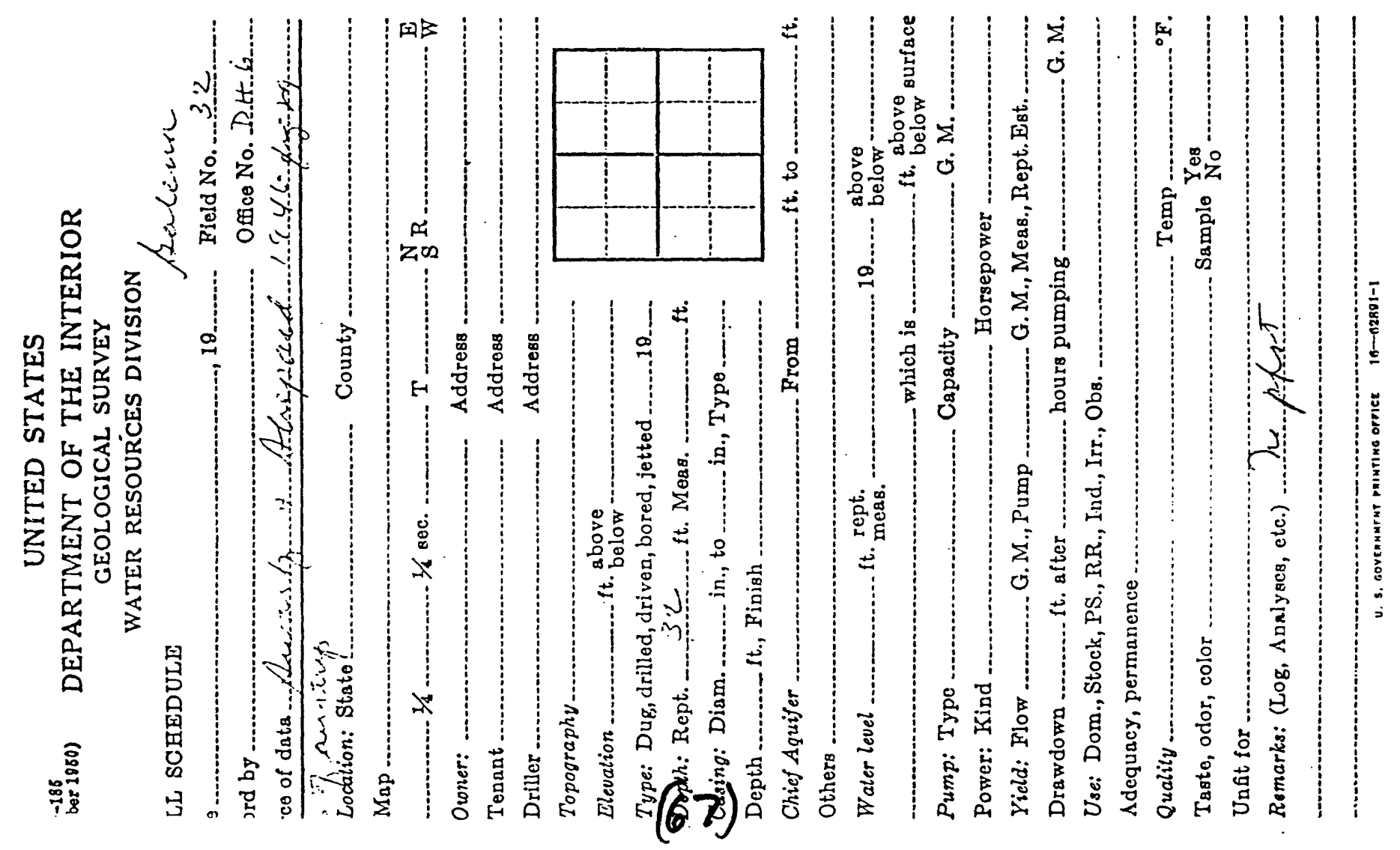




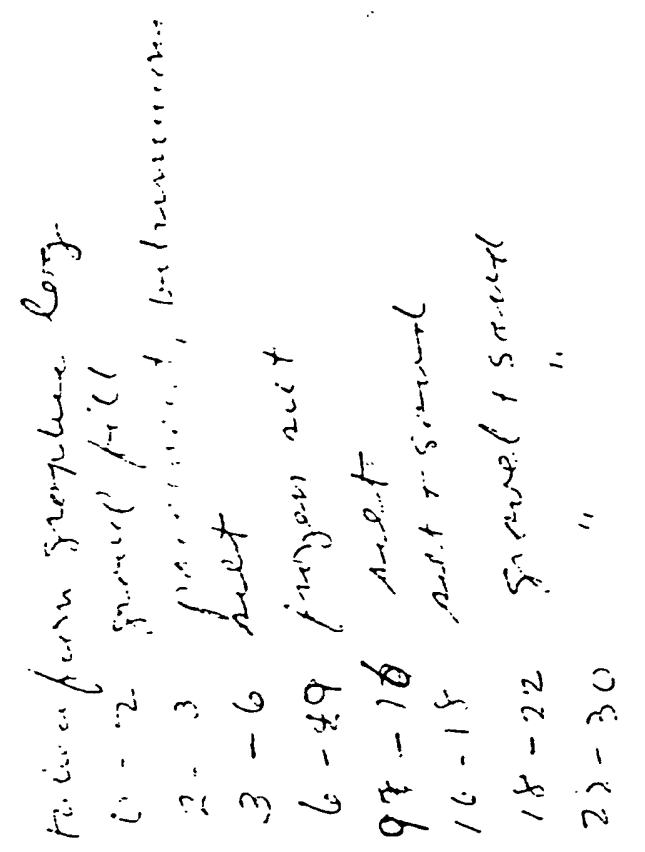

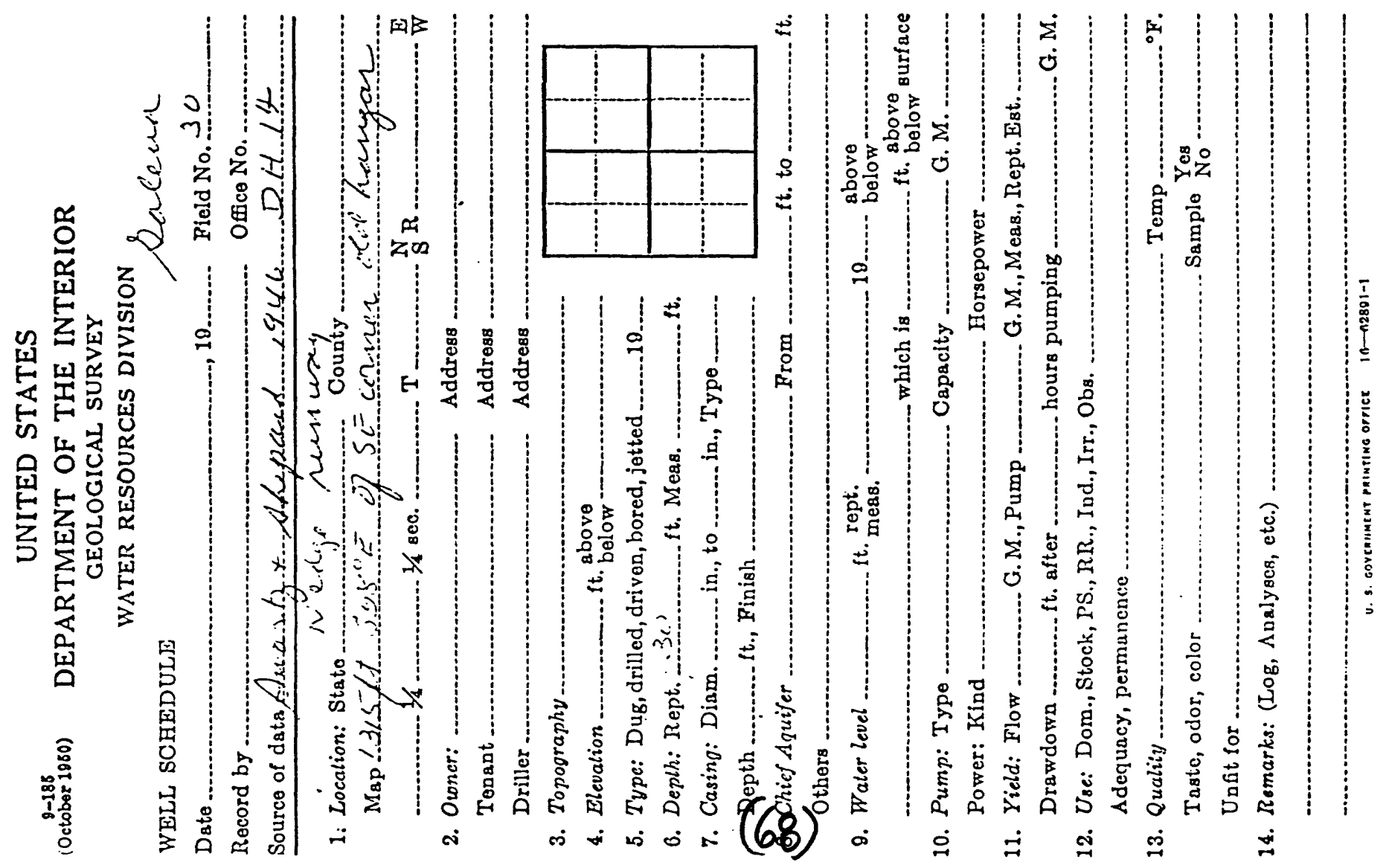



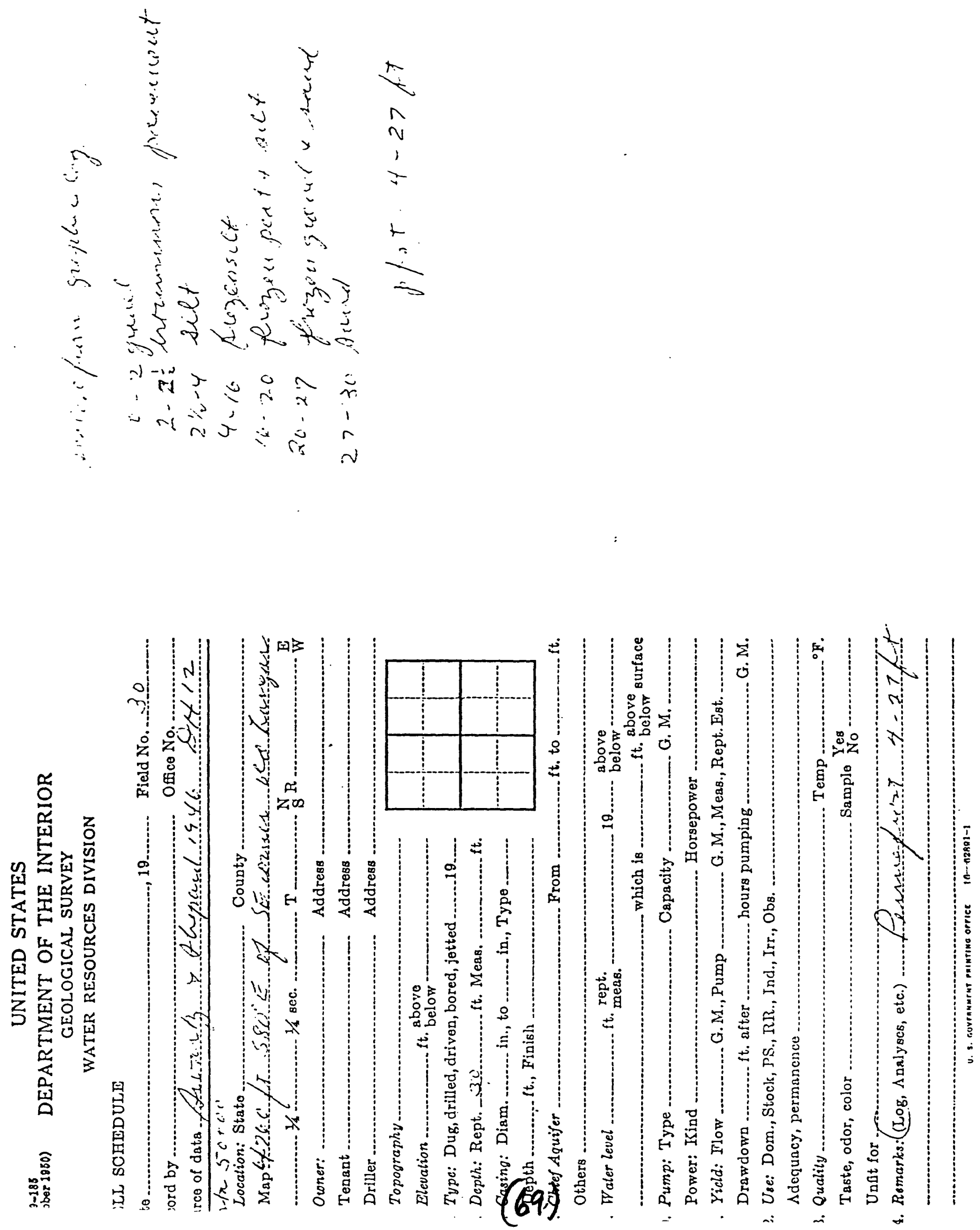

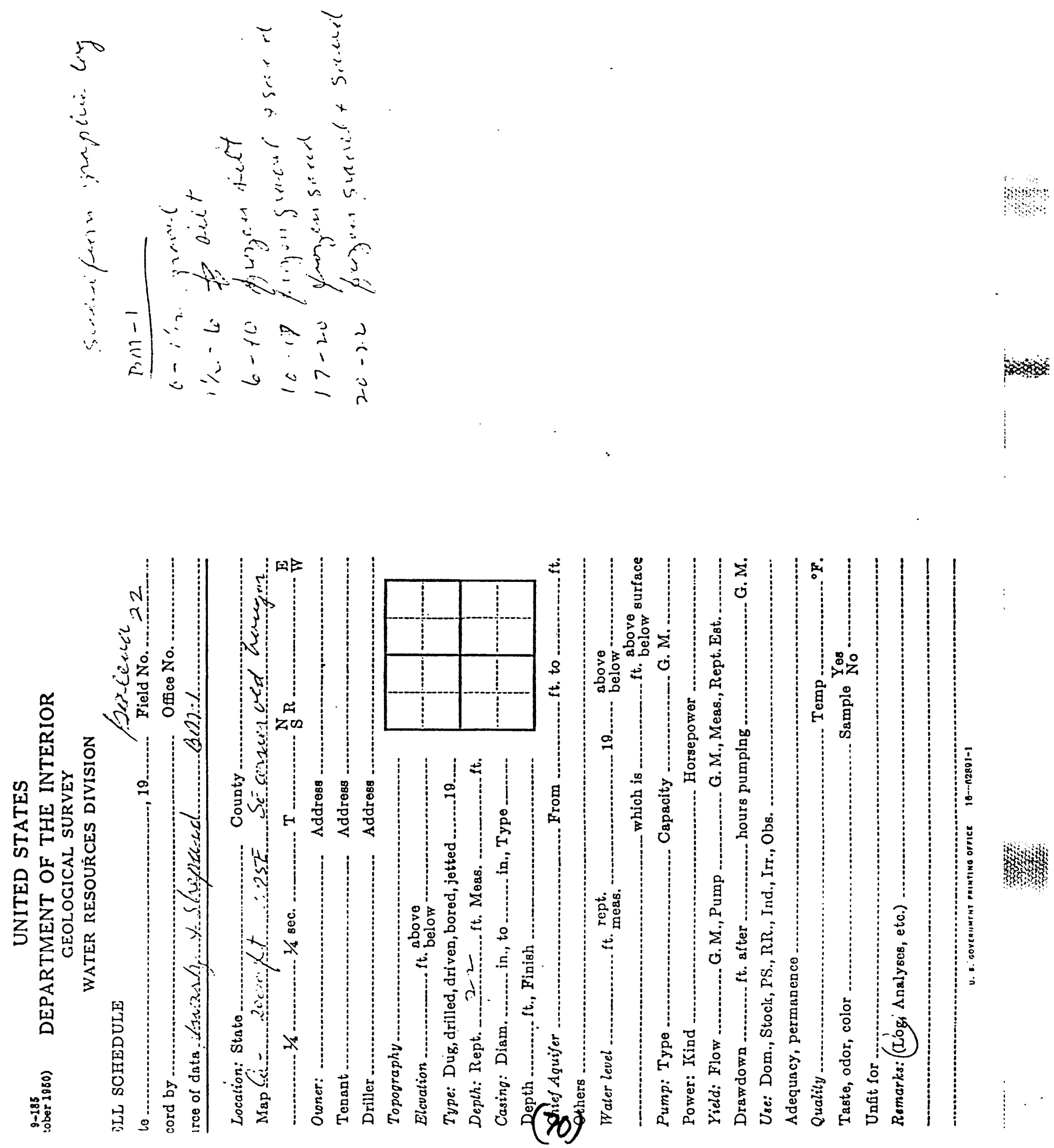


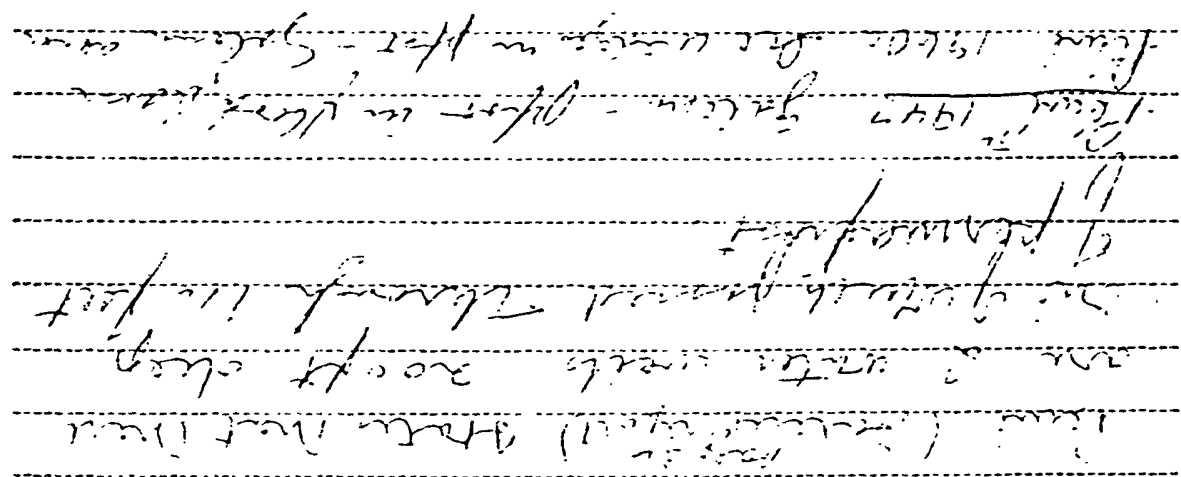

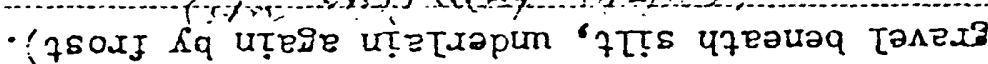

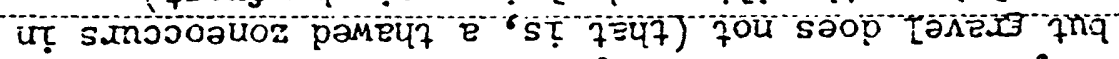

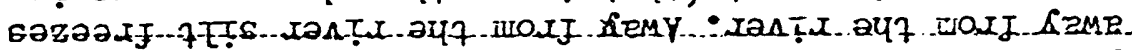

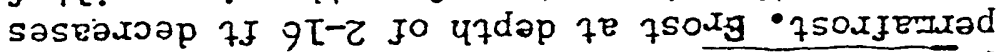
Jo $7 \mathrm{~J}$ OL पे

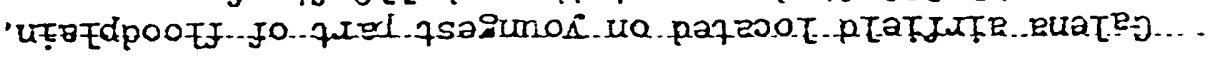

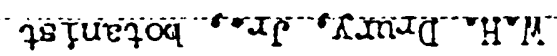

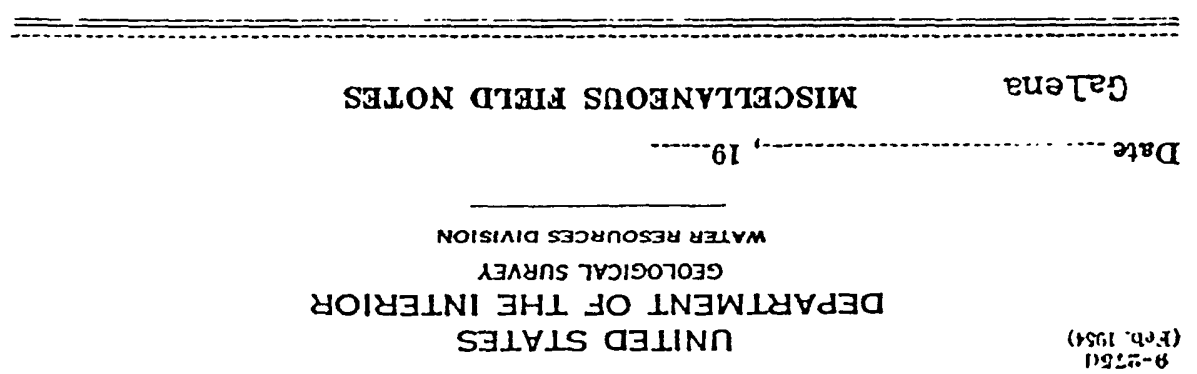


愘突

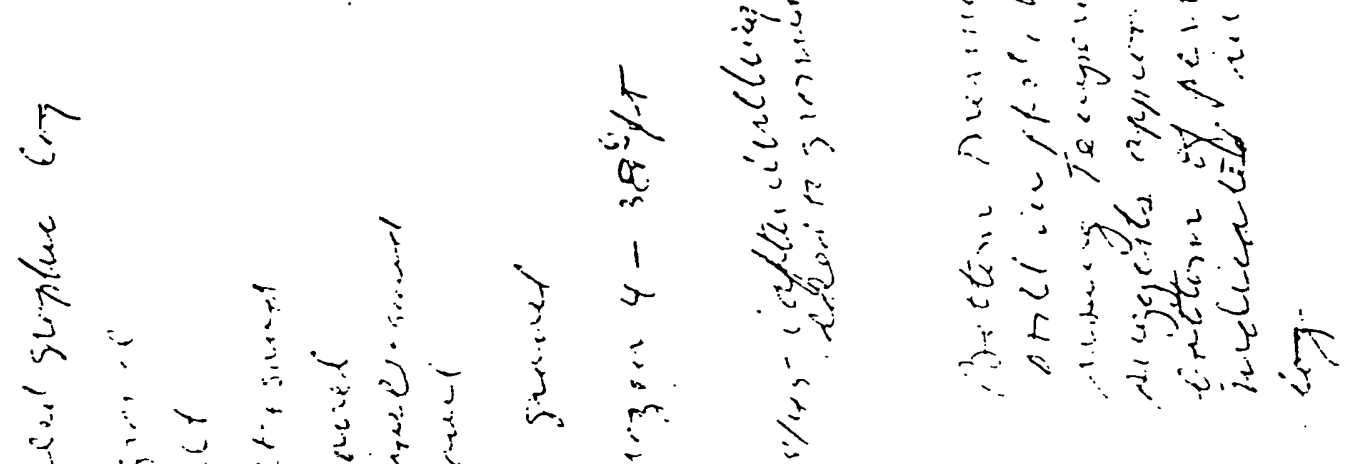

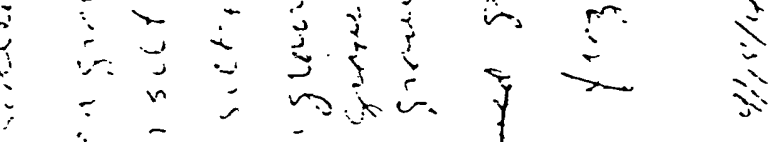

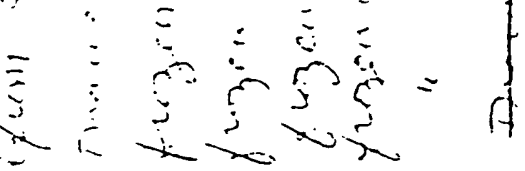

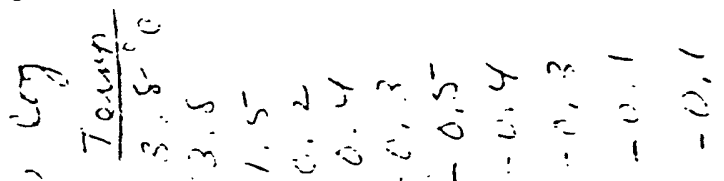

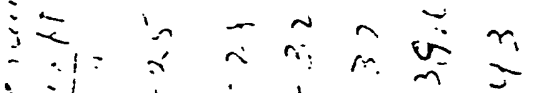

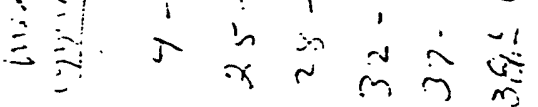

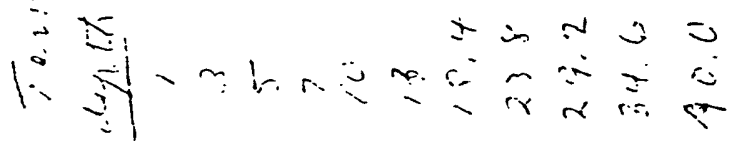

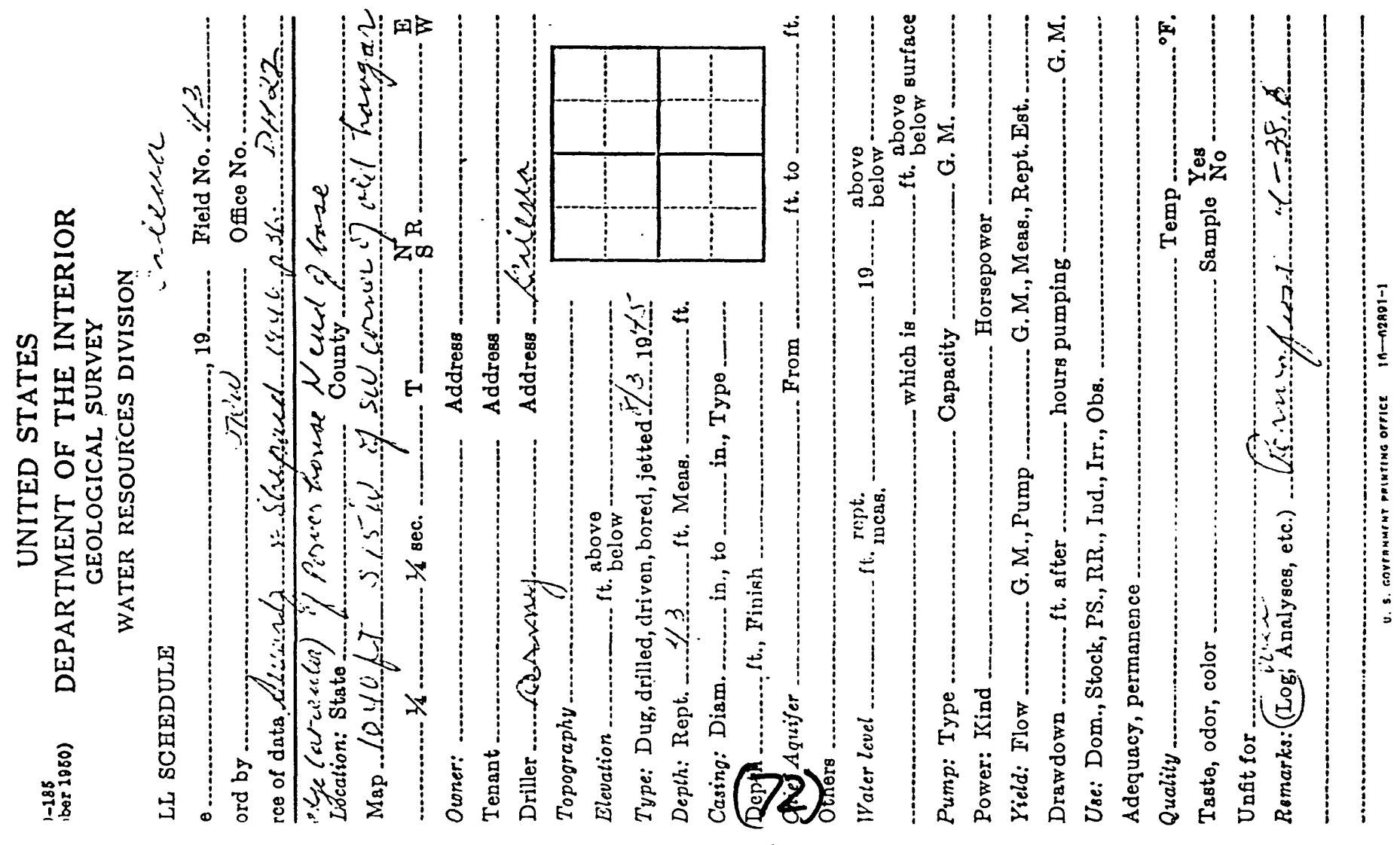



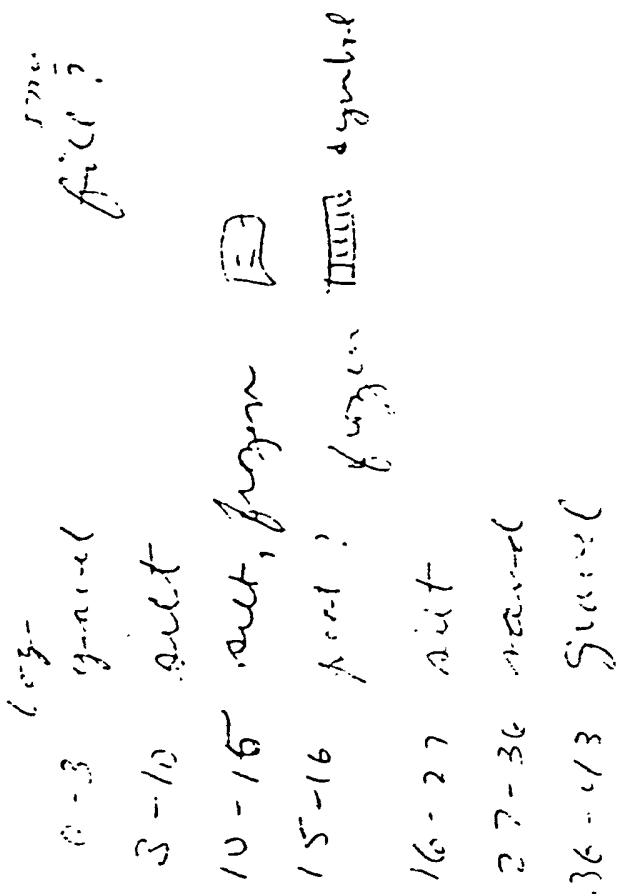

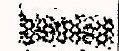

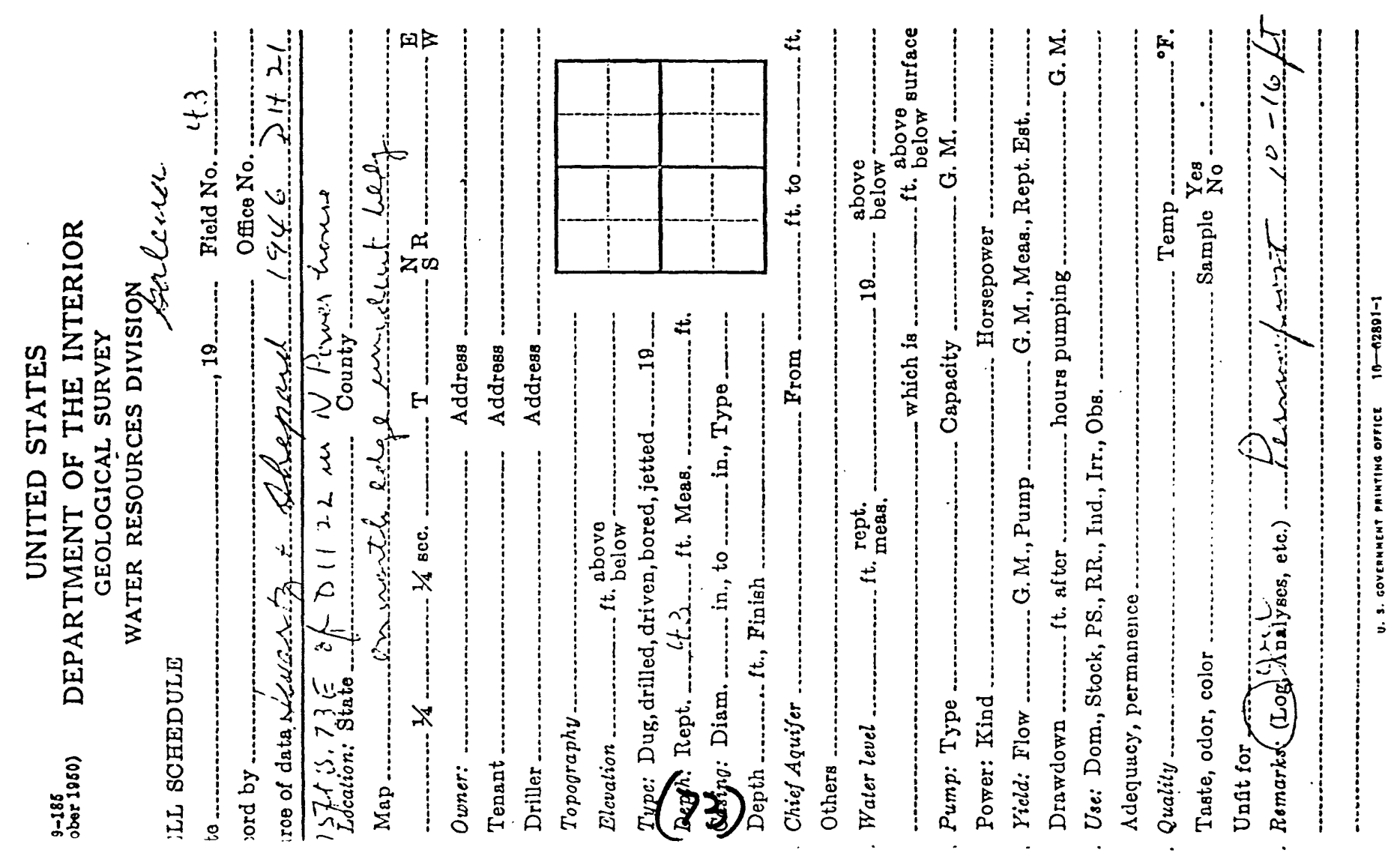


WATER RESOURCES DIVISION

Analyses by Geological Survey, United States Department of the Interior $9-268$ q (parts per million)

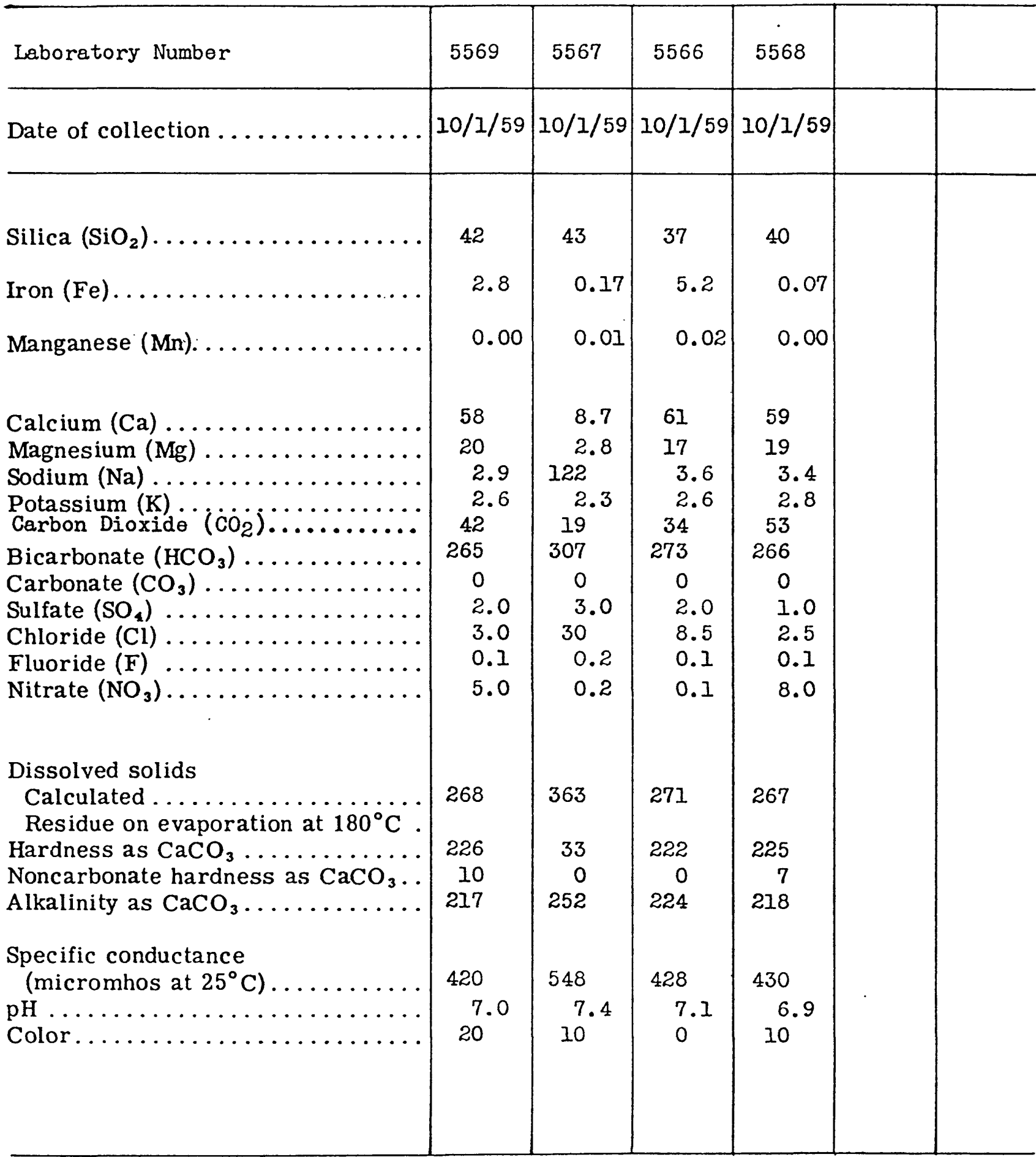

5569 - Galena Air Base, well \#2, raw water.

5567 - Galena Air Base, treated water, collected in water treatment plant.

5566- Galene Air Base, well 1, chlorinated water (elert hanger well).

5568:- Galena Air Base, well 3, raw water (fire station well).

Table .-- Chemical analyses of water from principal sources at Galena Air. Force Base. 
Location SOTADA ADS - Galune

Source Yukca

Cased to (ft) Date drilled Owner
County hth oltisted

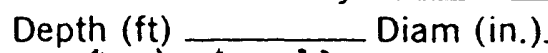

Treatment urtrogto:

WBF

Temp ( $\left.{ }^{\circ} \mathrm{F}\right)$

Collected

Remarks

\begin{tabular}{|c|c|c|c|c|c|}
\hline & $\mathrm{ppm}$ & epm & & ppm & epm \\
\hline Silica $\left(\mathrm{SiO}_{2}\right)$ & 邀 & & Bicarbonate $\left(\mathrm{HCO}_{3}\right)$ & 266 & 4.36 \\
\hline Aluminum (Al) & & & Carbonate $\left(\mathrm{CO}_{3}\right)$ & & \\
\hline Iron (Fe) (dts) & 0.09 & & & & \\
\hline Honignexe (tan) & 0.25 & & Sulfate $\left(\mathrm{SO}_{4}\right)$ & 2.0 & 0.02 \\
\hline & & & Chloride (Cl) & 0.5 & 0.01 \\
\hline . & & & Fluoride (F) & 0.2 & 0.01 \\
\hline Calcium ( $\mathrm{Ca})$ & 62 & 3.09 & & & \\
\hline Magnesium (Mg) & 25 & 1.27 & Nitrate $\left(\mathrm{NO}_{3}\right)$ & c.4 & 0.01 \\
\hline Sodium (Na) & $\ddot{2} . ?$ & 0.12 & & & \\
\hline Potassium (K) & $1 . ?$ & 0.04 & & & \\
\hline Total & & 4.52 & Total & & $4 \cdot 41$ \\
\hline
\end{tabular}

WL _ Yield

By

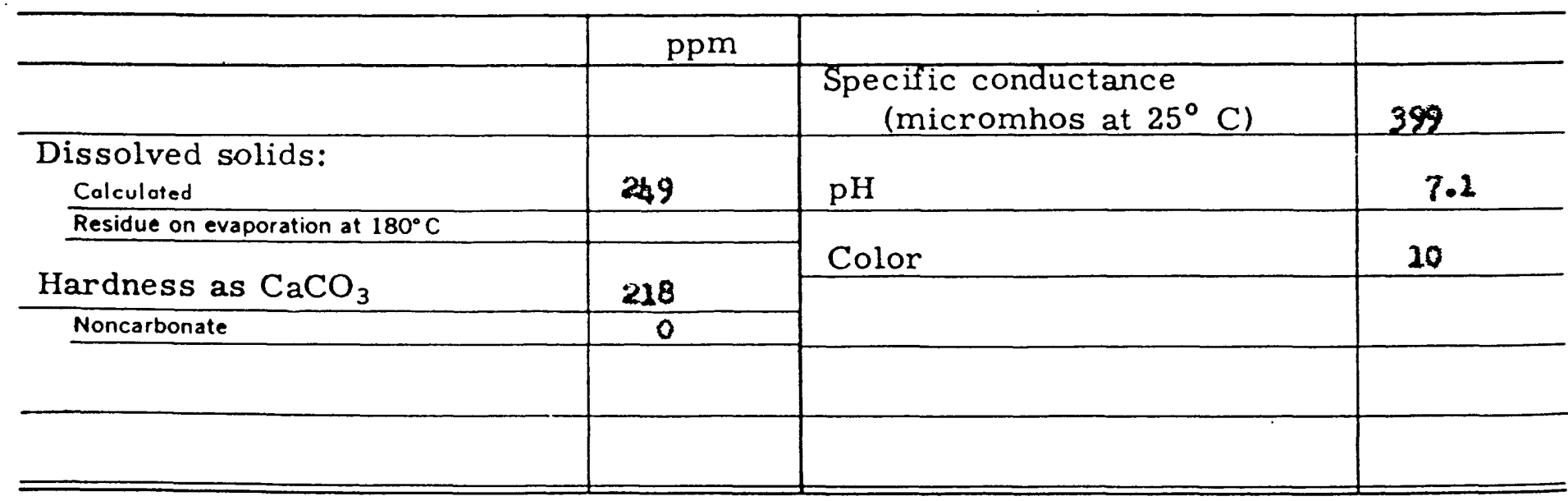

Lab. No. Col 6835

Field No.

Project 
Location $5072 n a ̈$ (fal

Source

Cased to (ft)
Yricon

Date drilled

Owner

Depth (ft) County 4th $^{\text {thindoson }}$ Diam (in.)

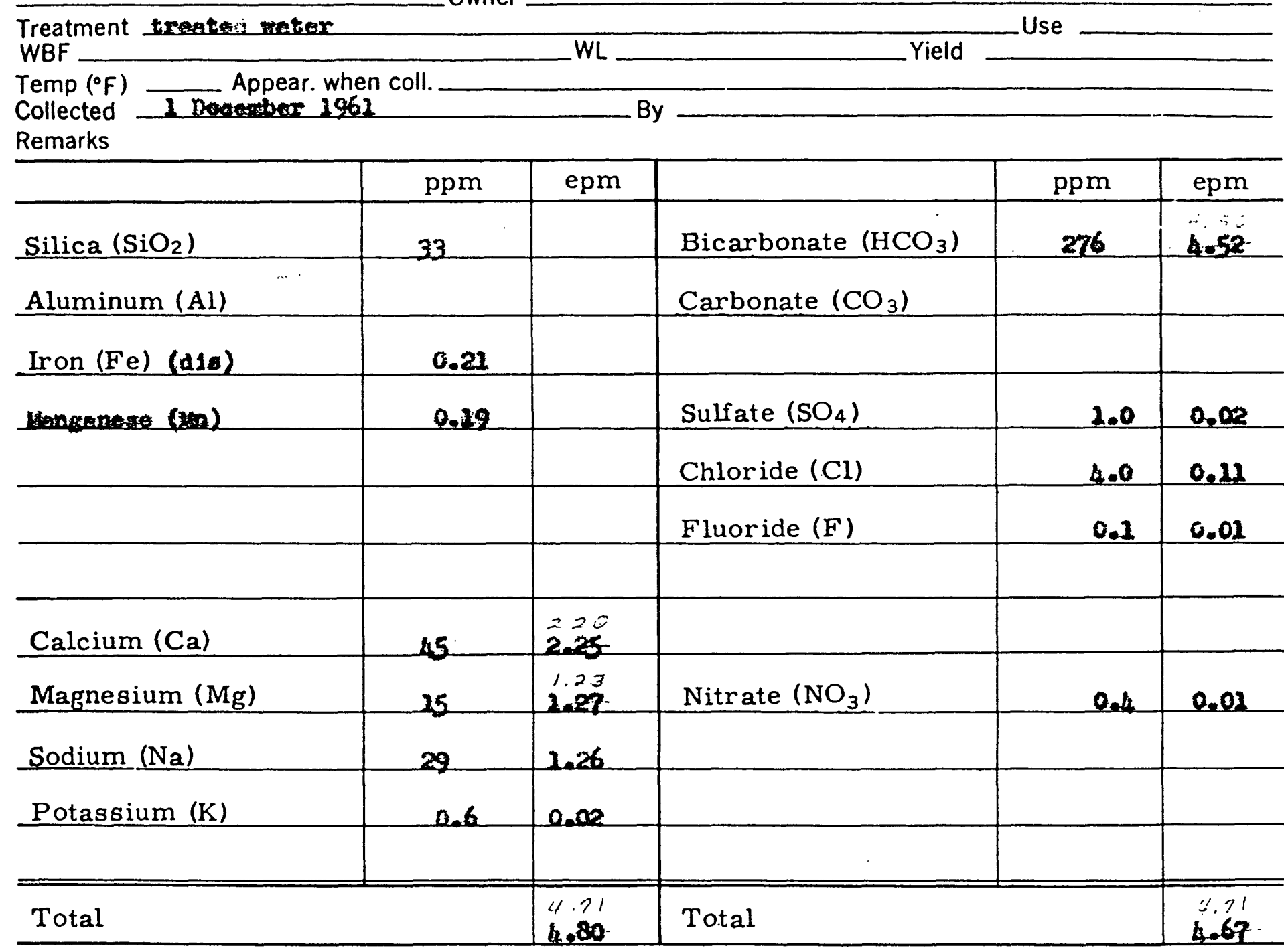

\begin{tabular}{|c|c|c|c|}
\hline & ppm & & \\
\hline & & $\begin{array}{l}\text { Specific conductance } \\
\left(\text { micromhos at } 25^{\circ} \text { C) }\right.\end{array}$ & 403 \\
\hline $\begin{array}{l}\text { Dissolved solids: } \\
\text { Colculated }\end{array}$ & $\frac{264}{286 x}$ & ( & 8.2 \\
\hline \multicolumn{2}{|l|}{ Residue on evaporation at $180^{\circ} \mathrm{C}$} & \multirow{3}{*}{ Color } & \multirow{3}{*}{20} \\
\hline \multirow{2}{*}{ Hardness as $\mathrm{CaCO}_{3}$} & \multirow[b]{2}{*}{176} & & \\
\hline & & & \\
\hline & & & \\
\hline & & & \\
\hline
\end{tabular}


Location 5UT a

Source Yakez:

Cased to $(\mathrm{ft})$

Treatment

WBF

Temp ( $\left.{ }^{\circ} \mathrm{F}\right)$

Collected

Remarks
Date drilled
Appear. when coll.

I Leotaber 1961 Owner

County \&tin if ision

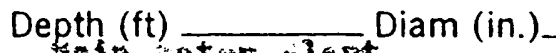

WL

Use

By

\begin{tabular}{|c|c|c|c|c|c|}
\hline & ppm & epm & & ppm & epm \\
\hline Silica $\left(\mathrm{SiO}_{2}\right)$ & 37 & & Bicarbonate $\left(\mathrm{HCO}_{3}\right)$ & 324 & $5 \cdot 11$ \\
\hline Aluminum (Al) & & & Carbonate $\left(\mathrm{CO}_{3}\right)$ & & \\
\hline Iron (Fe) (dis) & 0.76 & & & & \\
\hline \multirow[t]{3}{*}{ Mensanoso (iba) } & 0.02 & & Sulfate $\left(\mathrm{SO}_{4}\right)$ & 2.0 & 0.04 \\
\hline & & & Chloride (Cl) & 6.5 & 0.18 \\
\hline & & & Fluoride (F) & 0.2 & 0.01 \\
\hline Calcium (Ca) & 8.4 & 0.42 & & & \\
\hline Magnesium (Mg) & 2.4 & 0.20 & Nitrate $\left(\mathrm{NO}_{3}\right)$ & 2.1 & 0.02 \\
\hline Sodium (Na) & 205 & 4.57 & & & \\
\hline Potassium (K) & $2 \cdot 3$ & 0.0 .2 & & & \\
\hline Total & & 5.22 & Total & & $5 \cdot 36$ \\
\hline
\end{tabular}

\begin{tabular}{l|c|l|c}
\hline & ppm & & \\
\hline & & $\begin{array}{c}\text { Specific conductance } \\
\left.\text { (micromhos at 25 } 5^{\circ} \mathrm{C}\right)\end{array}$ & 465 \\
\hline $\begin{array}{l}\text { Dissolved solids: } \\
\text { Colculated }\end{array}$ & 318 & $\mathrm{pH}$ & $\mathbf{7 . 5}$ \\
\hline $\begin{array}{l}\text { Residue on evaporation at } 180^{\circ} \mathrm{C} \\
\text { Hardness as } \mathrm{CaCO}_{3}\end{array}$ & 31 & Color & 50 \\
\cline { 4 - 5 } Noncarbonate & 4 & & \\
\hline
\end{tabular}

Lab. No. Col 6831

Field No.

Project 
Location 5072nd ABD - Fulato suedrangle Source Yukcn

Cased to $(\mathrm{ft})$ Date drilled Owner

County isa divalos

Depth ( $\mathrm{ft}$ ) Diam (in.)

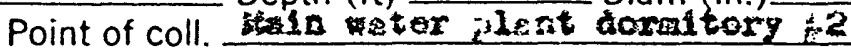

Treatment

WBF

WL

Use

Temp ( $\left.{ }^{\circ} \mathrm{F}\right)$

Collected

Appear. when coll.

clear +collection

Yield

By

Remarks

\begin{tabular}{|c|c|c|c|c|c|}
\hline & ppm & epm & & $\mathrm{ppm}$ & epm \\
\hline Silica $\left(\mathrm{SiO}_{2}\right)$ & 30 & & Bicarbonate $\left(\mathrm{HCO}_{3}\right)$ & 315 & 5.16 \\
\hline Aluminum (Al) & & & Carbonate $\left(\mathrm{CO}_{3}\right)$ & & \\
\hline Iron (Fe) (d10) & 1.0 & & & & \\
\hline \multirow[t]{3}{*}{ wanose (2m) } & 0.02 & & Sulfate $\left(\mathrm{SO}_{4}\right)$ & 2.0 & 0.03 \\
\hline & & & Chloride $(\mathrm{Cl})$ & 7.0 & 0.20 \\
\hline & & & Fluoride (F) & 0.2 & 0.01 \\
\hline Calcium (Ca) & 8.8 & 0.44 & & & \\
\hline Magnesium (Mg) & 2.9 & 0.24 & Nitrate $\left(\mathrm{NO}_{3}\right)$ & 0.6 & 0.01 \\
\hline Sodium (Na) & 205 & $\begin{array}{r}4.56 \\
456\end{array}$ & & & \\
\hline Potassium (K) & 1.7 & 0.04 & & & \\
\hline \multicolumn{2}{|l|}{ Total } & $\begin{array}{r}5.35 \\
5.28\end{array}$ & \multicolumn{2}{|l|}{ Total } & $\begin{array}{r}5.35 \\
-5.42\end{array}$ \\
\hline
\end{tabular}

\begin{tabular}{|c|c|c|c|}
\hline & ppm & & \\
\hline & & $\begin{array}{l}\text { Specific conductance } \\
\left(\text { micromhos at } 25^{\circ} \mathrm{C}\right)\end{array}$ & 468 \\
\hline $\begin{array}{l}\text { Dissolved solids: } \\
\text { Calculated }\end{array}$ & 322 & $e_{1-2}$ & 3.0 \\
\hline Residue on evaporation at $180^{\circ} \mathrm{C}$ & & Color & 60 \\
\hline $\begin{array}{l}\text { Hardness as } \mathrm{CaCO}_{3} \\
\text { Noncarbonate }\end{array}$ & $\frac{30}{0}$ & & \\
\hline & & & \\
\hline
\end{tabular}


WATER RESOURCES DIVISION

Analyses by Geological Survey, United States Department of the Interior $9-268 \mathrm{q}$ (parts per million)

\begin{tabular}{|c|c|c|c|c|c|c|}
\hline Loboretory Nunber & 6062 & 6853 & 6364 & 6365 & 6.366 & 6867 \\
\hline 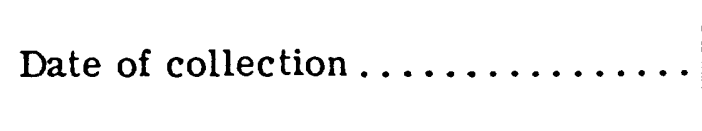 & $\begin{array}{l}29 \operatorname{Jan} \\
1962\end{array}$ & $\left\{\begin{array}{c}29 \operatorname{Jan} . \\
1962\end{array}\right.$ & $\begin{array}{c}29 \text { Jan. } \\
1962\end{array}$ & $\begin{array}{l}29 \text { Jan. } \\
1962\end{array}$ & $\begin{array}{l}29 \text { Jan. } \\
1962\end{array}$ & $\begin{array}{l}29 \text { Jan. } \\
1962\end{array}$ \\
\hline 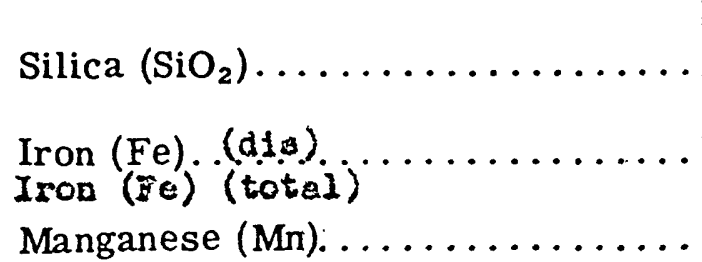 & $\begin{array}{l}38 \\
0.03 \\
5.6 \\
0.00\end{array}$ & $\begin{array}{l}38 \\
0.93 \\
2.6 \\
0.08\end{array}$ & $\begin{array}{l}33 \\
0.05 \\
5.5 \\
0.23\end{array}$ & $\begin{array}{l}33 \\
0.12 \\
16 \\
0.34\end{array}$ & $\begin{array}{l}32 \\
0.17 \\
12 \\
0.13\end{array}$ & $\begin{array}{l}39 \\
0.29 \\
1.5 \\
0.00\end{array}$ \\
\hline $\begin{array}{l}\text { Calcium }(\mathrm{Ca}) \ldots \ldots \ldots \ldots \ldots \ldots \\
\operatorname{Magnesium}(\mathrm{Mg}) \ldots \ldots \ldots \ldots \ldots \\
\operatorname{Sodium}(\mathrm{Na}) \ldots \ldots \ldots \ldots \\
\text { Potassium }(\mathrm{K}) \ldots \ldots \ldots \ldots \ldots \ldots \ldots \ldots\end{array}$ & $\begin{array}{l}263 \\
4=18 \\
\because 2.6 \\
\because 2.6\end{array}$ & $\begin{array}{l}744 \\
\therefore=8.0 \\
379 \\
=4.5\end{array}$ & $\begin{array}{l}648 \\
=26 \\
21 \\
=32.1 \\
4.72\end{array}$ & 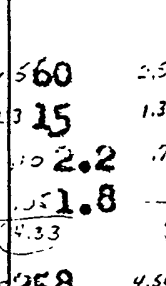 & $=\begin{array}{l}652 \\
216 \\
216 \\
=28 \\
320.9 \\
\div 6\end{array}$ & $\left\{\begin{array}{l}448.8 \\
.587 .2 \\
100 \\
3.1 \\
317 \\
317\end{array}\right.$ \\
\hline $\begin{array}{l}\text { Bicarbonate }\left(\mathrm{HCO}_{3}\right) \ldots \ldots \ldots \ldots \ldots \\
\text { Carbonate }\left(\mathrm{CO}_{3}\right) \ldots \ldots \ldots \ldots \ldots \ldots \ldots \\
\text { Sulfate }\left(\mathrm{SO}_{4}\right) \ldots \ldots \ldots \ldots \ldots \ldots \ldots \ldots \\
\text { Chloride }(\mathrm{Cl}) \ldots \ldots \ldots \ldots \ldots \ldots \ldots \ldots \\
\text { Fluoride }(\mathrm{F}) \ldots \ldots \ldots \ldots \ldots \ldots \ldots \\
\text { Nitrate }\left(\mathrm{NO}_{3}\right) \ldots \ldots \ldots \ldots \ldots \ldots \ldots \ldots\end{array}$ & $\begin{array}{l}=2687 \\
=21.0 \\
0=1.0 \\
\therefore 0.2 \\
-0.38\end{array}$ & $\begin{array}{l}3272 \\
=231.0 \\
2320 \\
=10.2 \\
0.8\end{array}$ & $\begin{array}{l}04 \\
0=1.0 \\
-4.0 \\
-0.1 \\
-0.2 \\
4.22\end{array}$ & $\begin{array}{l}-258 \\
=2.0 \\
0.1 .0 \\
-0.1 \\
-0.2\end{array}$ & $\begin{array}{l}275 \\
0=1.0 \\
.4 .0 \\
-0.1 \\
0.0 .4 \\
4.63\end{array}$ & $\begin{array}{l}317 \\
207.0 \\
010.2 \\
310.5 \\
5.44\end{array}$ \\
\hline $\begin{array}{l}\text { Dissolved solids } \\
\text { Calculated } \ldots \ldots \ldots \ldots \ldots \ldots . \ldots \\
\text { Residue on evaporation at } 180^{\circ} \mathrm{C} \\
\text { Hardness as } \mathrm{CaCO}_{3} \ldots \ldots \ldots \ldots \ldots \\
\text { Noncarbonate hardness as } \mathrm{CaCO}_{3} \ldots \\
\text { Alkalinity as } \mathrm{CaCO}_{3} \ldots \ldots \ldots \ldots . .\end{array}$ & 268 & $\begin{array}{r}290 \\
63 \\
\ldots\end{array}$ & $\begin{array}{c}264 \\
184 \\
-\infty\end{array}$ & $\begin{array}{l}241 \\
210 \\
\cdots\end{array}$ & $\begin{array}{l}260 \\
194 \\
\ldots\end{array}$ & $\begin{array}{r}323 \\
51 \\
-\end{array}$ \\
\hline 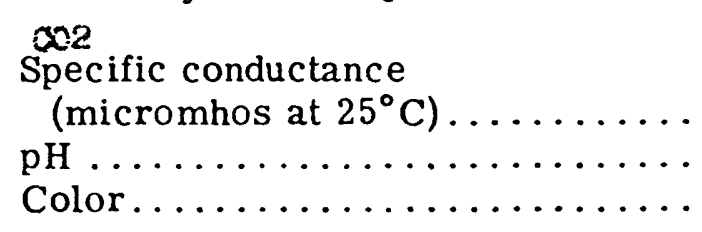 & $\begin{array}{l}46 \\
438 \\
7.0 \\
20\end{array}$ & $\begin{array}{c}u_{4} \\
460 \\
7.5 \\
60\end{array}$ & $\begin{array}{c}9 \\
429 \\
7.7 \\
10\end{array}$ & $\begin{array}{c}7 \\
409 \\
7.8 \\
20\end{array}$ & $\begin{array}{c}7 \\
409 \\
7.8 \\
20\end{array}$ & $\begin{array}{c}8 \\
482 \\
7.8 \\
30\end{array}$ \\
\hline
\end{tabular}

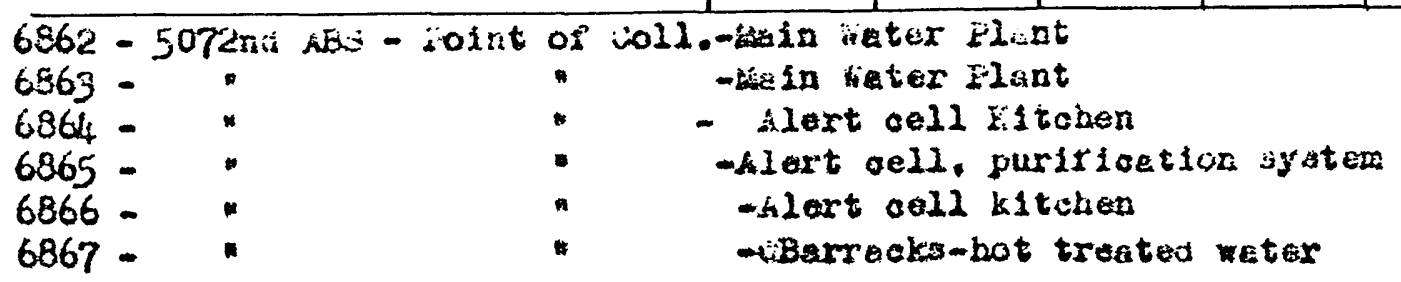

All otable - Ireatient 
U.S. DEPARTMENT OF THE INTERIOR

GEOLOGICAI SURVEY

WATER RESOURCES DIVISION

Analyses by Geological Survey, United States Department of the Interior $9-268 \mathrm{q}$ (parts per million)

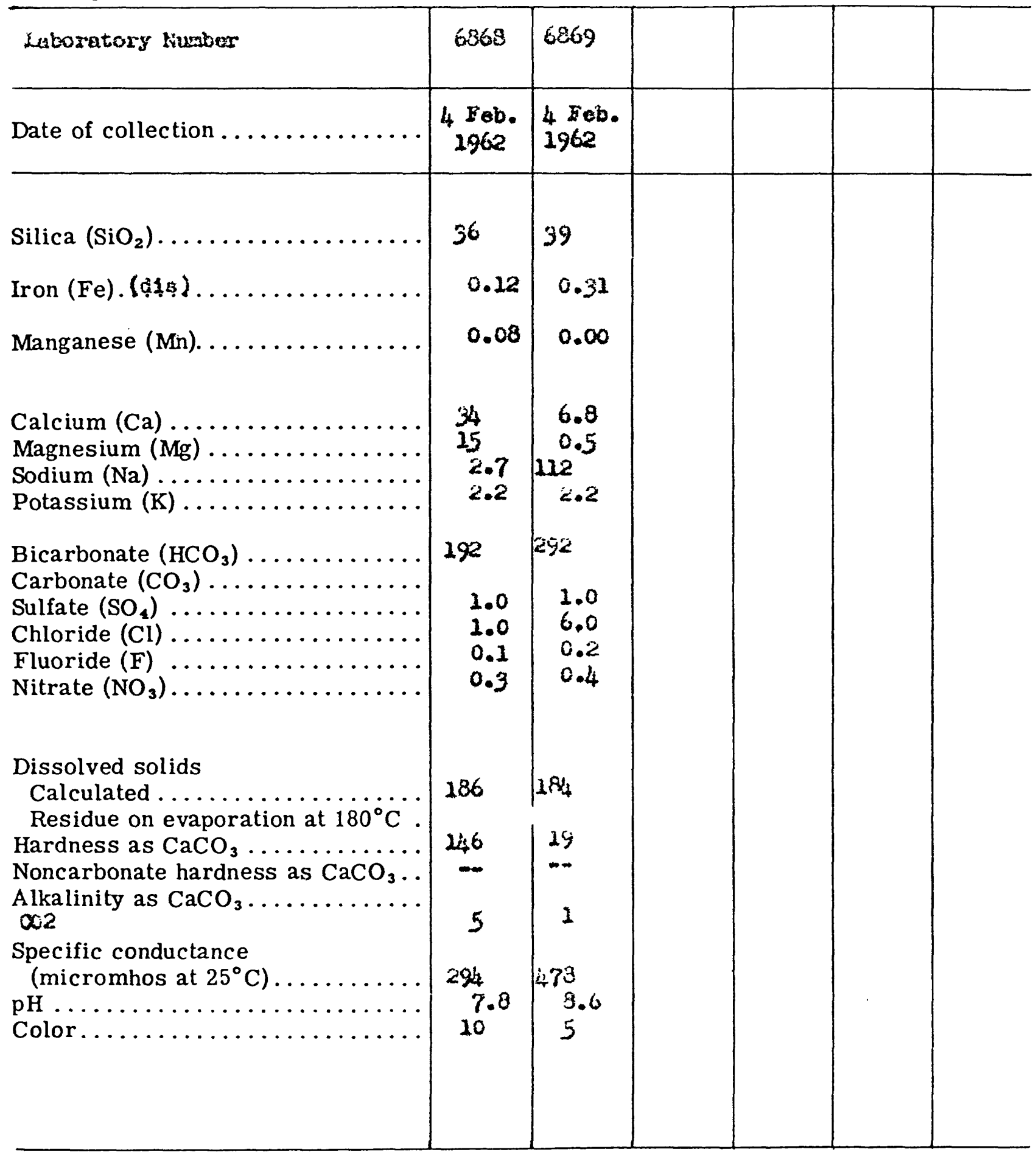

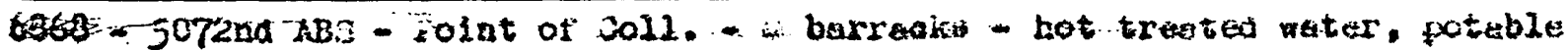
6869 .

- treated zater zoolite a fitratica tro tibent. i otabia 
U.S. DEPARTMENT OF THE INTERIOR

GEOLOGICAL SURVEY

WATER RESOURCES DIVISION

Analyses by Geological Survey, United States Department of the Interior $9-268 \mathrm{q}$ (parts per million)

Laboratory Number

Date of collection 5 kar. 5 suar. 5 viar.

Silica $\left(\mathrm{SiO}_{2}\right)$

Iron $(\mathrm{Fe})$. . (ass)

Manganese (Mn).

Calcium (Ca)

Magnesium (Mg)

Sodium (Na)

Potassium (K)

Bicarbonate $\left(\mathrm{HCO}_{3}\right)$

Carbonate $\left(\mathrm{CO}_{3}\right)$

Sulfate $\left(\mathrm{SO}_{4}\right)$

Chloride (Cl)

Fluoride (F)

Nitrate $\left(\mathrm{NO}_{3}\right)$

Dissolved solids

Calculated

Residue on evaporation at $180^{\circ} \mathrm{C}$.

Hardness as $\mathrm{CaCO}_{3} \ldots \ldots \ldots \ldots \ldots$

Noncarbonate hardness as $\mathrm{CaCO}_{3}$..

Alkalinity as $\mathrm{CaCO}_{3} \ldots \ldots \ldots \ldots$

Specific conductance

(micromhos at $25^{\circ} \mathrm{C}$ )

pH...

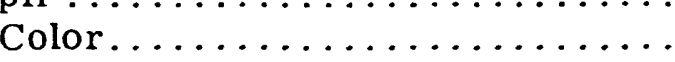

2962 


\section{U.S. DEPARTMENT OF THE INTERIOR GEOLOGICAL SURVEY}

WATER RESOURCES DIVISION so72nd ABS

Qalerta $4 F$

Galena, Alaske

Analyses by Geological Survey, United States Department of the Interior $9-268 \mathrm{q}$ (parts per million)

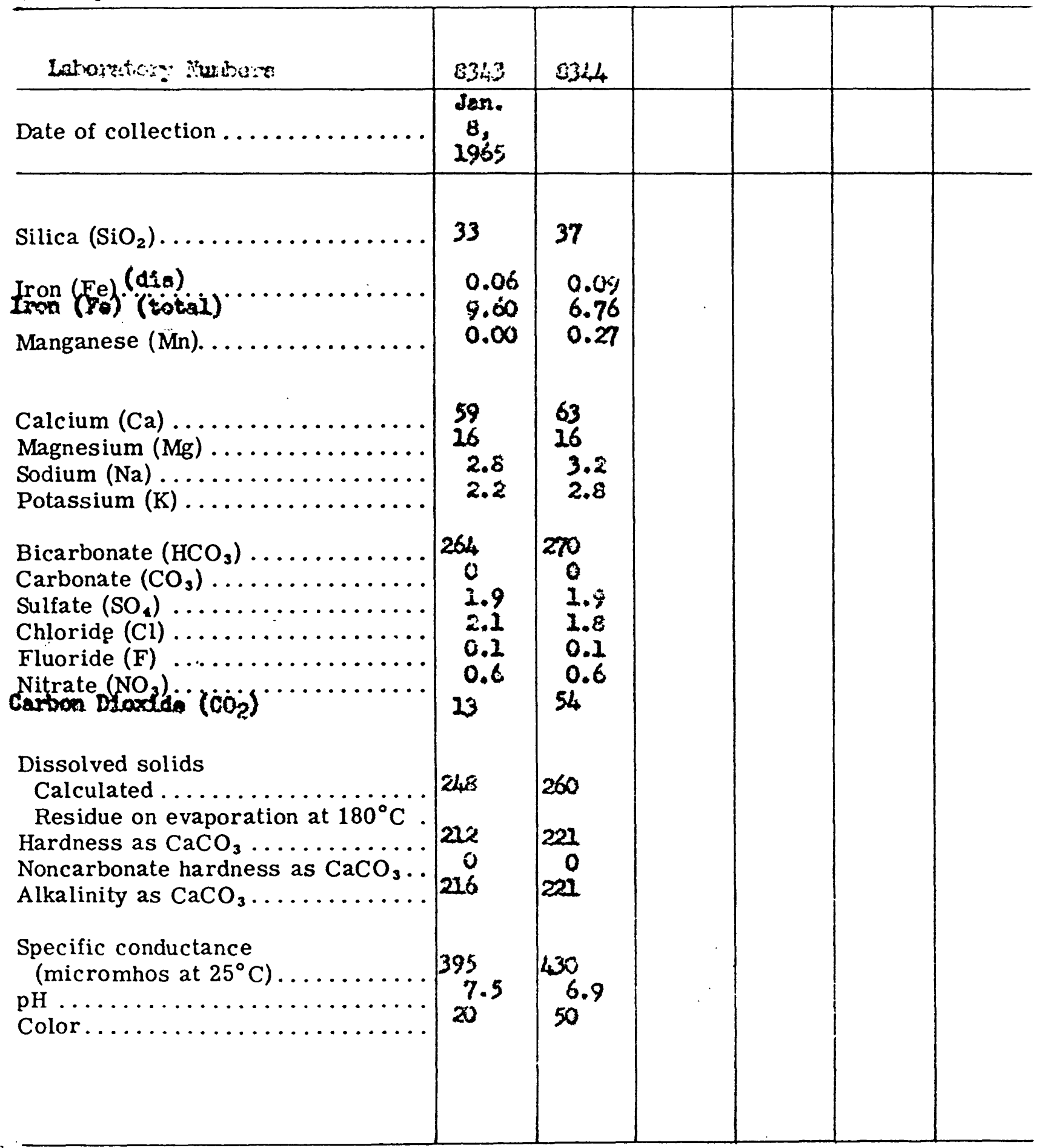

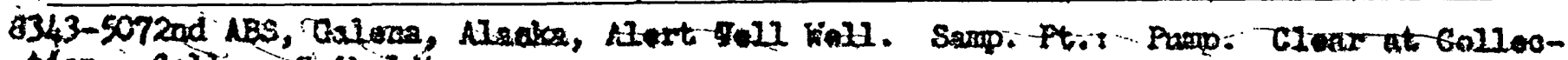
tion. Colli: selbaltt. 8344-5072and ABS, Celana, Alaska, Kein Plant. Samp. Pt. 3 befor degadrier. Clane at Colloction. Coll. s siboldt. Oaly opersting well for bsec ouppls. 
Location fि:

Source

Cased to $(\mathrm{ft})$

Date drilled

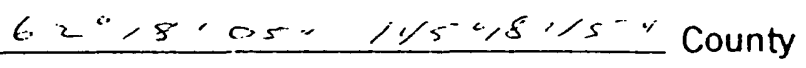
Depth (ft) Diam (in.)

Point of coll. Owner

Treatment

WBF

WL

Use

Temp A. Apear. when coll.

Collected Dotcon 3 , Ings

2 f3s houres Yield

Remarks

\begin{tabular}{|c|c|c|c|c|c|}
\hline & mpina & epen & & ppm & epan \\
\hline Silica $\left(\mathrm{SiO}_{2}\right)$ & $27^{11}$ & 11 & Bicarbonate $\left(\mathrm{HCO}_{3}\right)$ & 206 & $\begin{array}{l}90 / 3 \\
3.67\end{array}$ \\
\hline Aluminum (Al) & $\cdots$ & & Carbonate $\left(\mathrm{CO}_{3}\right)$ & 0 & .00 \\
\hline \multirow[t]{4}{*}{ Iron (Fe) } & .05 & & & & \\
\hline & & & Sulfate $\left(\mathrm{SO}_{4}\right)$ & 36 &.$n$ \\
\hline & & & Chloride (C1) & 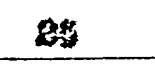 & .12 \\
\hline & & & Fluoride (F) & .2 & .02 \\
\hline \multicolumn{6}{|l|}{$i$} \\
\hline Calcium ( $\mathrm{Ca}$ ) & 60 & 3.20 & & & \\
\hline Magnesium (Mg) & 24 & 2.25 & Nitrate $\left(\mathrm{NO}_{3}\right)$ & .8 & .40 \\
\hline Sodium (Na) & $y$ & .8 & & & \\
\hline Potassium (K) & 3.8 & .30 & & & \\
\hline \multicolumn{2}{|l|}{ Total } & 0.35 & \multicolumn{2}{|l|}{ Total } & 0.08 \\
\hline
\end{tabular}

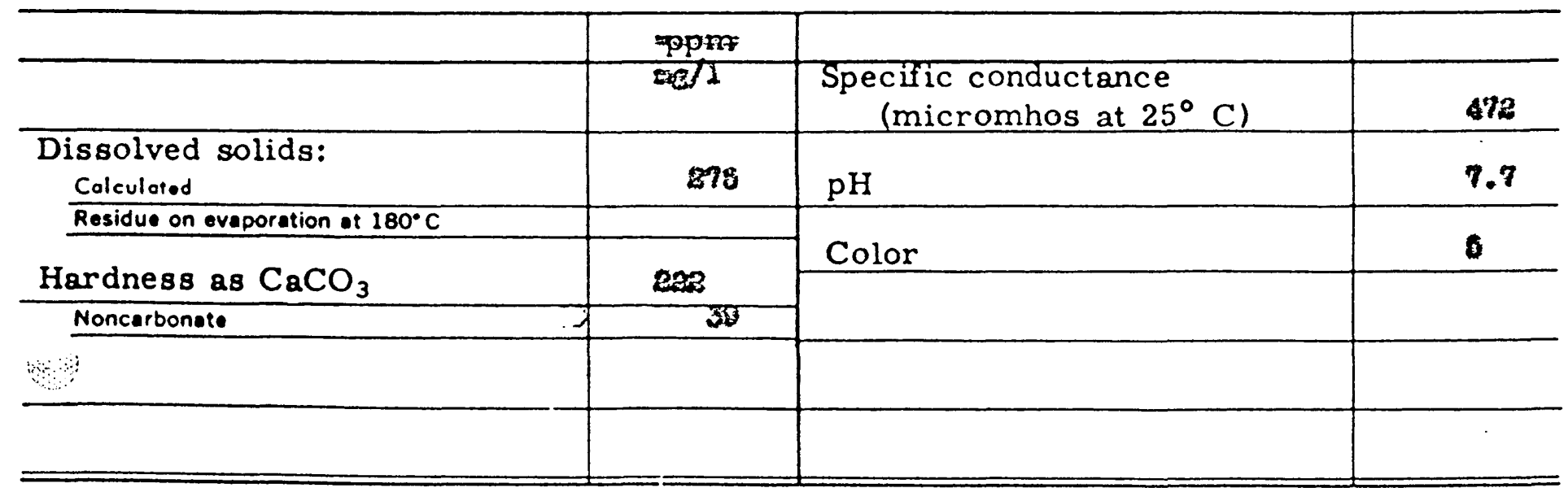

Lab. No. Col11577-63-04 Field No. 
S. NE'PAR'IME'NI' OF' THE INTERIOR

GEOLOGICAI SURVEY

WATER RESOIRCES DTUTISION

Analyses by Geological Survey, United States Department of the Interior $9-268 \mathrm{q}$ (parts per million)

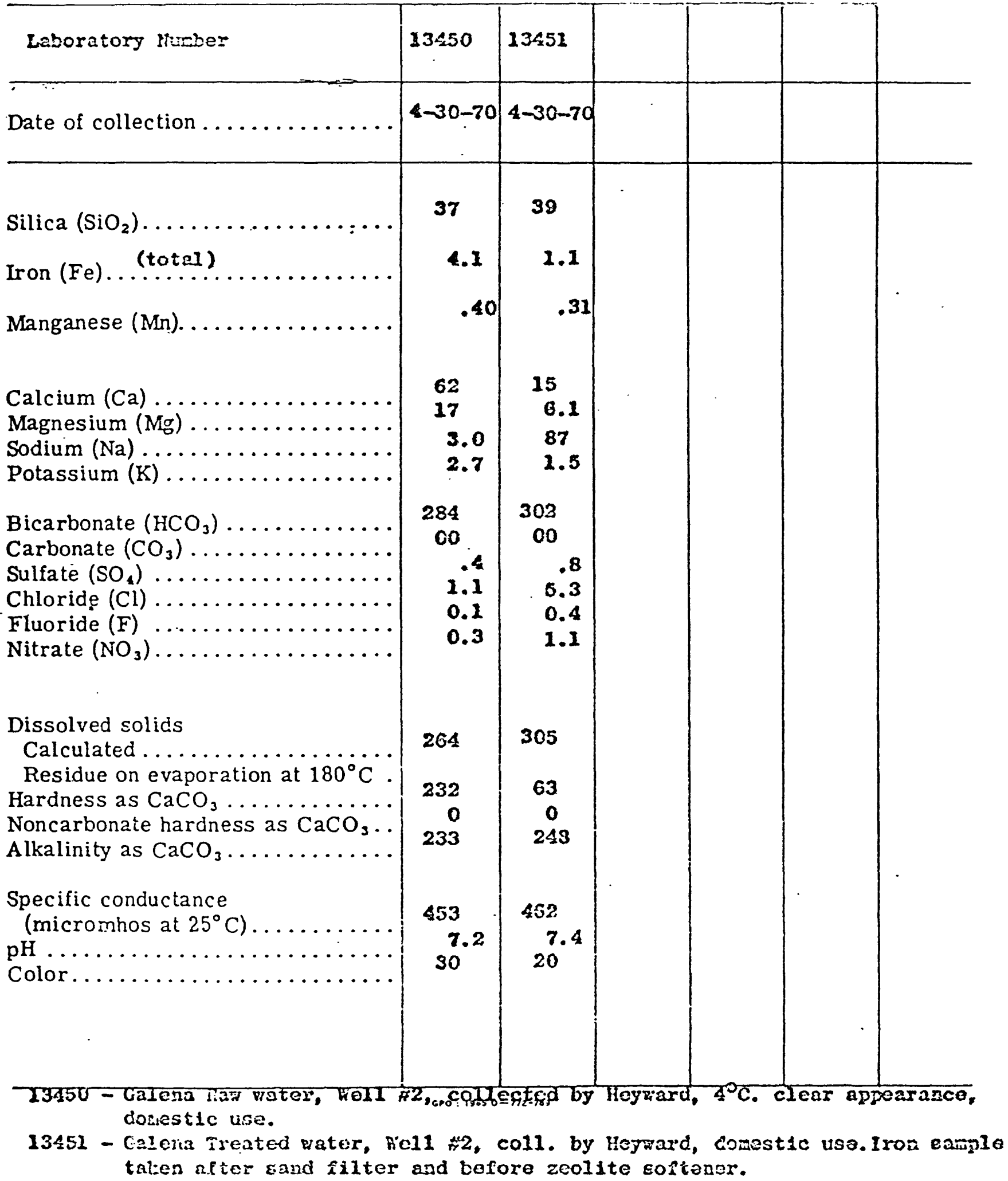


-.S. DEPARTMENT OF THE INTERIOR

GEOLOGICAL SURVEY

WATER RESOURCES DIVISION

Analyses by Geological Survey, United States Department of the Interior 9-268 q (parts per million)

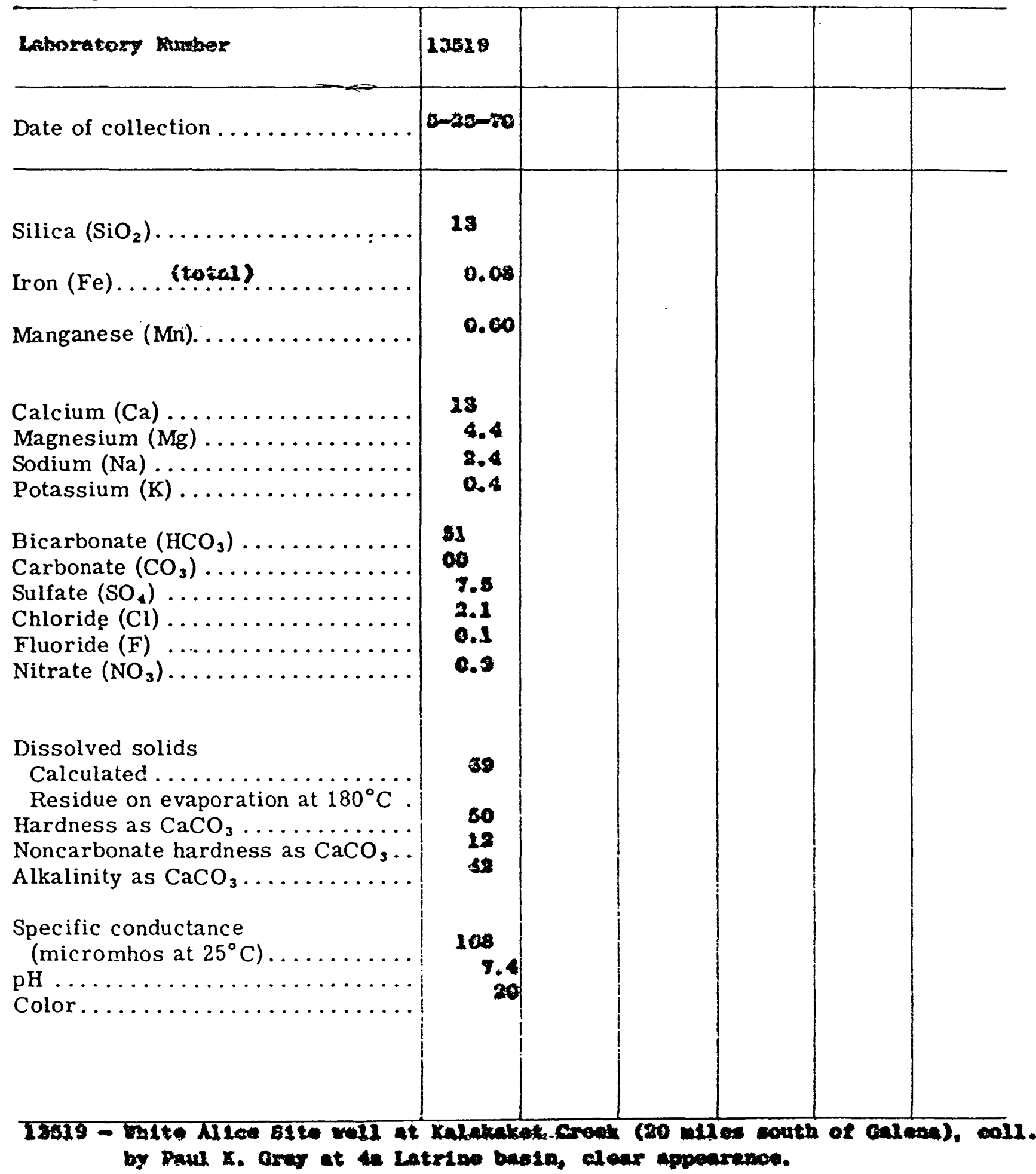




\section{APPENDIX 6}

\section{U.S. Army Corps of Engineers}

"Report on Galena airport observation wells Galena, Alaska" 


\section{REPORT ON \\ GALENA AIRPORT OBSERVATION WELLS \\ GALENA, ALASKA}

\section{Corps of Engineers \\ U. S. Army Engineer District, Alaska Anchorage, Alaska}

Prepared by

Foundations and Materials Bramch

10 May 1963 
1. INTRODUCTION
a. Scope of Investigation
b. Location . . . . . .
c. Previous Investigations.

2. GEOGRAPHY

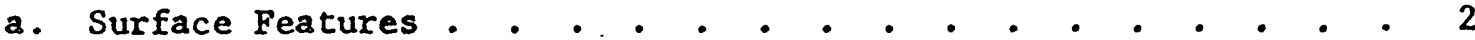

b. Climatology . . . . . . . . . . . . . . . . 3

c. Development of Area . . . . . . . . . . . . . . . 3

(1) Airport Facilities . . . . . . . . . . . . . . 3

(2) Transportation . . . . . . . . . . . . . . 5

(3) Water Supply Development . . . . . . . . . . . . 5

3. GEOLOGY

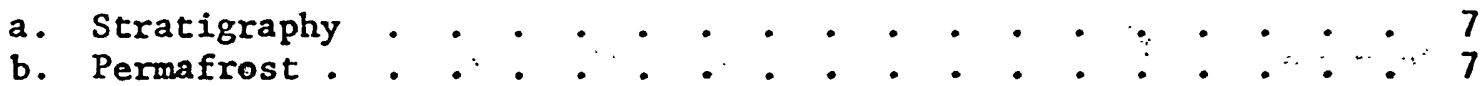

4. GROUND WATER

a. Source of Ground Watex - . - . - . - . . - . . . 8

b. Occurrence of Ground Water. . . . . . . . . . . . 8

5. OBSERVATION WELLS

a. Test Well No. W-345 . . . . . . . . . . . . . . . 9

b. Test Well No. W-346. . . . . . . . . . . . . . 10

c. Test Well No. W-347 . . . . . . . . . . . . . . . 11

d. Test Well No. W-348 . . . . . . . . . . . . . . . 13

6. CHEMISTRY OF GROUND WATER

a. Chemical Constituents . . . . . . . . . . . . . 14

b. Methods of Analysis . . . . . . . . . . . . . . . 15

7. TEST RESULTS

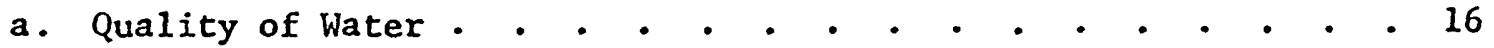

b. Quantity of Water. . . . . . . . . . . . . 18

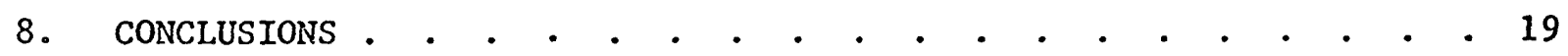

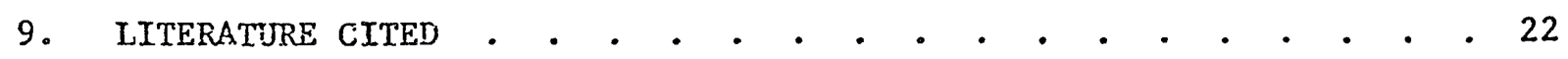

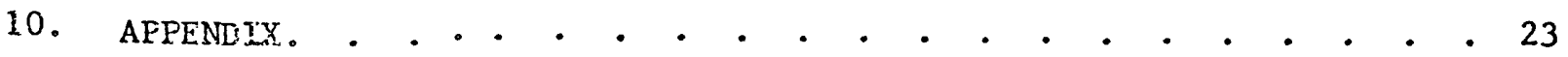


REPORT ON

GALENA AIRPORT OBSERVATION WELLS

GALENA, Alaska

\section{INTRODUCTION}

\section{a. Scope of Investigation}

Foundations and Materials Branch was advised on 2 August 1962

of a proposed project to construct five observation water wells at Galena Airport for the purpose of securing information on the quantity and quality of water that can be developed by an infiltration gallery. The proposal, submitted by Colonel Harold C. White, Assistant DCS/Civil Engineering, Alaskan Air Command, provided for the drilling and casing of five 6-inch diameter water wells not more than 40 feet in depth. Purpose of the work was to determine the feasibility of an alternate source of water at Galena Airbase. The proposal states in part that "The existing water supply is from wells and carries a high content of dissolved $\mathrm{CO}_{2}, \mathrm{FeO}$ and other undesirable chemicals which cause high corrosion of water lines and the steam heating system. According to preliminary studies by the U. S. Geological Survey there is a good possibility of developing a source of water of better quality and requiring less treatment than the existing well supply. This project is required to establish the quantity and quality of infiltrated water by means of observation wells. The work is to be performed by the ADE with their own forces and equipment. . . . Additionally, it is necessary that observation of the ground water be conducted over a period of at least one year to establish feasibility of this source of water." (USAF Project Justification Data Form 161, 
Dept. Identification No. GAL 72-3 (Rev. 1), Budget Account No. P-458(2525), proposed appropriation $\$ 11,400.00)$.

In addition to this proposal, a temporary electric line and well house would be provided for and accomplished by the Alaskan Air Command under a sork order request.

Design instructional Number $\mathrm{MC}-1 / 63-\mathrm{HZ17}-\mathrm{A} / \mathrm{CE} / 3$ dated 10 August 1962 was submitted by Alaskan Afr Command to Division Englneer, Portland.

Authority to accomplish work was granted by Division Engineer, Portland on 10 December 1962.

\section{b. Location}

Galena Airport is located in west central Alaska adjacent to the north bank of the Yukon River. The site is about 270 miles west of Faírbanks, 250 miles east of Nome, and on the direct alr route between these two cities.

c. Previous Investigations

Reports on the area include those of Eardley (1938 a, b) who commented upon the sediments and the meandering of the Yukon River, and Péwé (1948) who studied permafrost conditions at the Alrport. A number of unpublished reports concerning investigation of subsurface conditions for design and construction activities $2 t$ the Airport are avallable in the files of the Foundations and Materials Branch.

\section{GEOGRAPHY}

\section{a. Surface Features}

The lower Yukon River valley varies considerably in width and is. about 35 miles wide in the vicinity of the Galena Air Base. The valley 
is bounded by bedrock hills on three sides. The Yukon River flows westward in this broad valley and is joined by the Koyukuk River from the north. The valley contains numerous terraces and a wide, irregularly shaped floodplain. In most places the terraces are carved out of the enormous silt deposits in the valley. Gravel, sand and muck are found in lesser quantities. Two small bedrock hills, Bishop Rock and Pilot Mountain, respectively 13 miles and 11 miles west of Galena Airport, are the only protuberances on an otherwise broad, flat floodplain. The floodplain is irregularly shaped and ranges in width from less than 1 mile to a maximum of about $2 q$ miles. From the air, the floodplain appears as a complicated series of sloughs, meander scars, oxbow lakes, creeks, tundra and forest. (Péwé, 1948). Original elevation of the east-west rumway of 93 to $98 \mathrm{ft}$ has been raised by fill and pavement to $128.0 \mathrm{ft}$ MSL:

\section{b. Climatology}

The mean annual temperature at Galena is $23.7^{\circ} \mathrm{F}$. Mean temperature for month of January is $-11.4^{\circ} \mathrm{F}$. and for July $59.5^{\circ} \mathrm{F}$. Annual precipitation is 13.6 inches including 55.1 inches of snowfall. Climatological data are summarized in Table 1.

\section{c. Development of Area}

\section{(1) Airport Facilities}

The airfield is situated on low ground and in the summer months is only a few feet above the normal water level. At the time this airfield site was decided upon, recognition was given the fact that the field would be subject to occasional inundation during the spring floods, but the urgency of a field in this area forced acceptance of location. 
Floods occur each spring, the flood of 1944 being the best documented. The floods are the result of (1) abnormally high seasonal precipitation and (2) ice jams caused by huge blocks of ice piling up at bends and narrow sections of the river to form a dam. In the spring of 1944 such an ice jam occurred 40 miles below the Galena airfield. Aerial bombardment of the ice jam with 500 -pound bombs proved ineffective, and the field was flooded. Water stood seven feet deep in many of the huts and warehouses. It was decided at that time that some flood protection was needed. Plans included filling an area to a point above the normal flood elevations, this area to be in the immediate vicinity of the landing field and of a size large enough to accomodate all the necessary buildings. In addition, a dike was to be constructed around the entire landing field and camp area. This work was largely completed during 1944 and 1945. The dike was eventually built to a crest elevation of $135 \mathrm{ft}$ MSL. Silt was pushed out of borrow pits into a bump to form the dike. A permeable gravel bed underlies the dike and permits water from the river to "boll" up Into borrow pits during part of the spring and summer. Most seepage enters through borrow pits on the southwest, and in a drainage ditch between the runway and hangar. (Péwé 1948). In 1959, 1960, 1961 and 1962, piles were driven along the bank of the Yukon River to retard erosion. This erosion is an imediate threat to the southeast corner of the dike. Erosion continues to be a problem at the present time, and may eventually force relocation of the airport. The airport facilities include an eastwest runway, hangar, FAA commications transmitter, storage tanks for fuel and water, pumphouse, fire station, storage sheds, housing, chapel, and other facilities. 


\section{(2) Transportation}

Due to its isolated location, there are but two practical means of transportation to Galena. One is by air and can be used the year round; the other is by barge and boat from the railheat at Nenana, down the Tanana River to its junction with the Yukon, thence down the Yukon to Galena, a distance of 360 miles. River transportation can only be used during the months of June, July, August, and September and is afforded by small, shallow-draft river boats and barges.

\section{(3) Water Supply Development}

Water consumption at Galena AFS in 1961 was 18,688,000 gallons

per year (Feulner, 1962). In July 1954, F\&M Branch made a thorough search for data regarding the water wells existing at Galena (Smith, 1954). At that time there were four known wells that had been pumped or were being pumped. Two of the wells were in use furnishing water for the CAA and Air Force units. Except for Well No. 1, original construction data, logs, drawdown tests, and similar technical information could not be located in the files of either the CAA, AIO, AAC, USARAL, or the ADE. The location of the following wells is shown in Figure 2 :

Well No. 1 is in a small pumphouse on the east side of the fire station near the hangar. In 1954, it was the primary source of water for the base. It is a 6 -inch diameter well, $210 \mathrm{ft}$ in depth. The static water level in 1954 was $19.6 \mathrm{ft}$ below the surface.

Well No. 2 is located in Building T-120-B (called Water Treatment Bldg) just south of the CAA Fuel Oil Storage Building. The depth 
of the well is not known but is undoubtedly around $200 \mathrm{ft}$, since it was drilled by the same contractor about the same time as Well No. 1 . Static water level in July 1954 was at $19.6 \mathrm{ft}$. Very little change (4" to 5") in the level of the water was measured during pumping. At the present time this is, the main well supplying water to the Air Force station.

Well No. 3 is situated back of the CAA Fuel Storage Bldg (Corps of Engineer's Bldg T-211). This well is used only for fire protection. Well No. 4 was used from 1943 to 1946 for the old heat and power plant in the north area. It was situated in the old boiler house No. 2, Bldg T-309, but is now abandoned.

In June 1954 the CAA drilled a 4-inch diameter well near the Control Bldg. This well is designated in this report as CAA Well No. 4. The well was drilled to a depth of 43 feet; water was encountered at $23 \mathrm{fr}$ and the static water level was at $14.6 \mathrm{ft}$ depth. The well was pumped at $10 \mathrm{gpm}$ for $70^{\circ}$ hours and at the conclusion of the pumping test the water level was at $19.6 \mathrm{ft}$. This well is presently in use. Another well, CAA Well No. 5, was drilled in June 1954 adjacent to the fire station to a depth of $64 \mathrm{ft}$ but has since been abandoned. These two wells were drilled in an attempt to obtain water with a lower content of iron. It was believed that water from shallow depths would contain less iron than that obtained frow Well Nos. 1 and 2, which obtain water from deeper levels. However, samples of water from CAA Well No. 4 proved to be high in iron (Smith, 1954).

A permanent well is installed at the Alert Hangar but has no numerial designation. The well was drilled in October 1955 by the Air Force under private contract, and is $210 \mathrm{ft}$ deep. The well is separate 
and supplies the Alert Hangar only. The water is chlorinated but is not treated.

Water treatment facilities are housed in Bullding $\mathrm{T}-120-\mathrm{B}$ in which Well No. 2 is located. The facilities for water treatment include degasifiers which oxidize ferrous iron to ferric iron, pressure filters using sand to strain out silt particles and iron oxides in solid form, and zeolite softeners for removing calcium and magnesium. The water is chlorinated and pumped into a 10,000 gallon storage tank from which the station water supply is drawn.

\section{GEOLOGY}

\section{a. Stratigraphy}

The airport is constructed on gray micaceous silt underlain by sand and gravel. The thickness of the silt is about $1 \mathrm{ft}$ at the west end of the fleld, and increases toward the east to more than $16 \mathrm{ft}$. The top of the gravel layer rises slightly to the west and was exposed in the bottom of borrow pits at the west end of the field in 1948. The gravel layer is exposed along the river on the southwest and west sides of the field.

\section{b. Permafrost}

In 1948, the airport was underlain by a layer of permafrost $110 \mathrm{ft}$ tbick; the overlying active layer (seasonally frozen ground) was quite variable in thickness; the depth to permafrost was 3 to $4 \mathrm{ft}$ at the east end of the field and more than $16 \mathrm{ft}$ at the west end (Péwé, 1948).

Drillers of CAA Well No. 4, drilled to depth of $43 \mathrm{ft}$, and CAA Well No. 5, drilled to $64 \mathrm{ft}$, apparently did not report any permafrost. It is possible, but not probable, that the main permafrost table in 1954 
had receded to a depth of at least $64 \mathrm{ft}$ as far north as the power plant and fire station. Cross-sections prepared by Péwé (1948) show that the depth to permafrost decreases away from the river.

4. GROUND WATER

\section{a. Source of Ground Water}

The major source of recharge to shallow wells at Galena Alrport is considered to be the Yukon River. Some additional recharge to deep wells probably occurs by normal ground water movement from intake areas at or near the slopes of the surrounding highlands. Recharge to wells by means of downward percolation from the land surface in the immediate vicinity of the airport may not occur in significant amounts because of the relatively impermeable silt mantling the surface.

\section{b. Occurrence of Ground Water}

The chief water-bearing formation is the alluvium beneath the. permafrost layer at permanent Wells No. 1 and 2 . The absence of well logs does not permit any estimation to be made of the character of this alluvium, but it seems quite probable that the material consists of gravelly sand, sandy gravel, or interbedded sand and gravel layers.

Water also occurs between the top of the main permafrost table and the base of the seasonally frozen surface layer. Weli logs for CAA Wells No. 4 and 5 indicate this material as "gravel and sand" and "sand". The water table appears to be at a depth of approximately 19 to $24 \mathrm{ft}$ in these wells. 


\section{OBSERVATION WELLS}

The following data are a summary of the methods, procedures and results of the present program involving the drilling of four observation wells. This program took place from 21 February 1963 to 24 Apr 111963. Location of the test wells is shown in Figure 2. Two drillers equipped with a truck-mounted Star 71 churn drill with 6-inch bit arrived in Galena on 21 February 1963. The drillers met with Major Linton, Base Civil Engineer and Mr. Russell, Assistant Civil Engineer to discuss the location of the first well at the Ammo Storage Bldg and to comply with security regulations on the station.

\section{a. Test We11 No. W-345}

This well was located $41.0 \mathrm{ft}$ east of the Ammo Storage Bldg at coordinates $\mathrm{N} 101,481$; E 101,976. Elevation of ground surface at the well is about $120 \mathrm{ft}$. The location of the well was confirmed on 25 February 1963 and the well spudded in on the same day. Drilling was completed on 1 March 1963 at a depth of $50.0 \mathrm{ft}$. On 2 March 1963, the well was bailed for one hour. On 4 March 1963 a Johnson Everdur No. 30 slot screen, 5 ft 8 inches long with an 0.D. of 5-1/2 inch was set in the well with the bottom of the screen at $39 \mathrm{ft} 6$ inches. The static water level was at $30 \mathrm{ft} 6$-inches depth. The well was developed by bailing for $4-1 / 2$ hours as the static water was too close to the top of the screen to allow proper surging. The water cleared well but bad a slightly turbid appearance. Production was estimated at 8 to $10 \mathrm{gpm}$. It is noted that only $2.0 \mathrm{ft}$ of the screen was exposed due to failure to pull the casing up far enough. 
The pump used in making a pumping test at Test Well No. W-345 and subsequent tests at other holes was a Peerless Hi-lift submersible, $3 / 4 \mathrm{HP}, 115 \mathrm{~V} ; 1$ phase electric pump $2.4 \mathrm{ft}$ in overal1 length. On 6 March 1963, a water sample was taken for Bob Schupp of the U. S. G. S., Palmer. A two-hour pumping test was run on 8 March 1963 in which the water cleared we11; three water samples were taken, one at the beginning of the test, a second after forty-five minutes, and a third after two hours at the end of the test. On 21 March 1963, the screen was removed from the well and a start was made on pulling the casing. Hot water was required to loosen the casing. On 22 March 1963, hot water treatments were continued and the casing was eventually removed. The well was then abandoned.

\section{b. Test Well No. W-346}

Well No. W-346 is located near the southeast corner of the Guardhouse at coordinates N 101,501; E 101,702. Elevation of ground surface at the well is approximately $120 \mathrm{ft}$. On 11 March 1963, the well was spudded in and three ft drilled. Drilling to $19 \mathrm{ft}$ was completed on 12 March 1963; to $27 \mathrm{ft}$ on $13 \mathrm{March}$ 1963, and to $37 \mathrm{ft}$ on $14 \mathrm{Mar} 1963$. On 14 March a water sample was taken by Mr. Willis Morris of Alaskan Air Command at 28 - $29 \mathrm{ft}$. On 15 March 1963, after overnight settling, a water sample at $37 \mathrm{ft}$ was taken by Mr. Morris; drilling then continued to $46 \mathrm{ft}$. Permafrost was excountered at $42.5 \mathrm{ft}$. On 16 March 1963 drilling was completed at a depth of $56 \mathrm{ft}$. On 18 March 1963 a Johnson Everdur No. 30 slot I.D. Screen $5 \mathrm{ft}$ 9 inches in length was set in the hole with the screen bottom at $54 \mathrm{ft}$ and the casing pulled back so that 5 ft 0 inches of the screen was exposed. 
The static water level was found to be at $27 \mathrm{ft} 6$-inches depth. The hole was bailed for three hours. On 19 March 1963 the well was surged for $1-1 / 2$ hours with a dart bailer. A pumping test of $1 / 2$ duration was carried out at $6 \mathrm{gpm}$ because the pump would stop running at a lesser rate. The $6 \mathrm{gpm}$ rate was excessive and the drawdown could not be stabilized. Water samples were taken at the beginning and end of the test. On 24 April 1963 a Myres $1 / 2$ HP Jet pump, Serial No. 46062 , was set in the well. The bottom of the strainer on the screen is at a depth of $51 \mathrm{ft} 1$-inch below the ground surface, the water intake is at $48.0 \mathrm{ft}$, and the bottom of the airline is at $50 \mathrm{ft}$ 9-inches. The well was tagged "Surface water - may be contaminated". At the request of Mr. Willis Morris, Alaskan Air Command, DE personnel prepared suggestions for attaching the pump to the existing facilities. These suggestions are given in Figure 1.

\section{c. Test Well No. W-347}

Well No. W-347 is located on the north side of the pumphouse lean-to on the north side of the Alert Hangar; the coordinates are $N$ 101,314; E 97,558. Elevation of the ground surface at the hole is approximately $120 \mathrm{ft} \mathrm{MSL.} \mathrm{Hole} \mathrm{was} \mathrm{spudded} \mathrm{in} \mathrm{on} 23$ March 1963 and $7 \mathrm{ft}$ drilled. Drilling continued on 25 - 26 March 1963 and was completed or 27 March 1963 at a depth of $35 \mathrm{ft} 6$-inches. On 27 March 1963 a Johnson Everdur No. 30 slot I.D. screen 5 ft 9-inches long was set in the well with the bottom of the screen at $34 \mathrm{ft}$. Static water level was at $26 \mathrm{ft} 9$-inches depth. The well was balled for four hours. Water samples were taken at depths of 30 to $35 \mathrm{ft}$ and the samples were field tested for iron by Alvin J. Fuelner, U.S.G.S. 
On 28 March 1963 the well was surged and bailed for 8-1/2 hours. At 1300 hours, 29 March $1963 \mathrm{Mr}$. R. J. Velikanje arrived at Galena to observe a pump test of this well. During work on this well considerable time was lost owing to Air Force restrictions in the area resulting from spillage of jet fuel and special exercises. On 30 March 1963 the well screen was found to be at a depth of $35 \mathrm{ft}$, having settled one foot since originally placed. The screen was exposed for a length of $5.0 \mathrm{ft}$. On 1 April 1963 a pump test was made using a Peerless turbine with 3-inch column and 5-inch bowls. Static water level was at $26.9 \mathrm{ft}$ at start of test. The water level indicator, however, would not go past the 5-inch bowls, so drawdown could not be determined. The well was pumped at 28 to $34 \mathrm{gpm}$ for 24 hours Water samples were taken at intervals of $1 / 2,8,12$ and 20 hours, and at the conclusion of the test. The well would appear capable of yielding. about $20 \mathrm{gpm}$ at a stabilized drawdown level. On 20 April 1963 the screen was raised to a new setting with bottom of screen at 31 ft 4 inches depth and a 10-12 gpm jet pump installed. Naphtha, paint thinner, and jet fuel were noted on the ground at the site; the Fire Department hosed off the area but it was still dangerous to use a welding torch.

Static water level on 22 April was 26 ft 4 -inches from top of the asphalt paving. The well was developed by using a 3-1/2-inch by $21 \mathrm{ft}$ sand pump. The screen settled 5 inches during development to a depth of $31 \mathrm{ft} 9$ inches from top of asphalt paving. A Myres 3/4 HP 2-pipe jet pump with 1 inch by $1-1 / 4$ inch outlets was installed in the well. The depth to the bottom of the screen 
on the pump is $33 \mathrm{ft} 2$ inches (Elev $89.33 \mathrm{MSL}$ ) below the top of the casing which extends 1 ft 6 inches above asphalt paving; the depth to the end of the airline is $32 \mathrm{ft} 10$ inches (Elev $89.66 \mathrm{ft} \mathrm{MSL}$ ); and the depth to the end of the low water cut-off is $32 \mathrm{ft} 6$ inches (Blev $90.0 \mathrm{ft}$ MSL). The well was tagged "Surface water - may be contaminated".

\section{d. Test Well No. $\mathrm{W}-348$}

Well No. W-348 is located on the east side of the Water Treatment Bldg T-120B; the coordinates are N 101,936; $\mathrm{E} 98,162$. The elevation of the ground surface at this well is approximately $120 \mathrm{ft}$ MSL. Drilling began on 4 April 1963 and continued on 5 April 1963 to a depth of 32.5 ft at which point water was encountered for the first time. The hole was bailed and the static water level stabilized at $29 \mathrm{ft}$. A pumping test was made using an F\&M submersible pump, with the pump intake at $31.4 \mathrm{ft}$. Pumping at the rate of $5 \mathrm{gpm}$ was continued for three hours with a drawdown of $1.5 \mathrm{ft}$. Two water samples were taken. On 6 April 63 drilling was continued to a depth of $34.2 \mathrm{ft}$. A second pump test was carried out at the rate of two gpm for two hours with the pump intake at $32.7 \mathrm{ft}$; drawdown was $3.2 \mathrm{ft}$ with static level at $29 \mathrm{ft}$. Three water samples were taken. On 8 April $1963 \mathrm{drilling}$ continued to $36.2 \mathrm{ft}$. A third pump test was carried out at 4 gpm for 3 hours with the pump intake at $33 \mathrm{ft}$; drawdown was $2.1 \mathrm{ft}$ with static level at $29 \mathrm{ft}$. Five water samples were taken during the pump test. On 9 April 1963 drilling was completed at $40.2 \mathrm{ft}$. A fourth pump test was carried out at 7-1/2 gpm for 3 hours with the pump intake at $33 \mathrm{ft}$; drawdown was $3 \mathrm{ft}$ with static water level at $29 \mathrm{ft}$. Two water samples were taken. On 10 April a fifth pump test was carried out for 3 hours; three water samples were taken. 
On 12 April 1963 a No. 30 slot Johnson Everdur screen $5.7 \mathrm{ft}$ in length was set in the well with the bottom of the screen at $34 \mathrm{ft}$ depth. The casing was pulled back to the $30.5 \mathrm{ft}$ depth exposing $3.5 \mathrm{ft}$ of the screen and the screen packer was swedged against the inside of the casing. Upon completion of the screen installation, the top of the casing was found to be $2.0 \mathrm{ft}$ above the ground surface. The static water level on 13 April 1963 was at $29 \mathrm{ft}$ depth. On 13 April 1963 the pump was replaced in the well with the water intake at a depth of $32 \mathrm{ft}$, and the well was test pumped for the sixth time, for a period of $3-1 / 2$ hours. The drawdown was $2.1 \mathrm{ft}$ at a pumping rate of $4 \mathrm{gpm}$. Water samples were taken for the District Testing Laboratory at the beginning, at $1-1 / 2$ hours, and at the completion of the pumping test. A complete chemical analysis of this well: is given in Table 5.

\section{e. Pumps}

(1) The pump used in Testing Well No. W-348 was withdrawn from the well at request of Air Force and stored in the Galena Air Force Base electrician's shop and was capped with a welded seal. This pump is as follows: Fair anks Morse submersible $1 / 2 \mathrm{HP}, 115$ volt, single phase, 9.4 amp $1.60 \mathrm{SF}$, 60 cycle, Model C-4004B23DI complete with 42 gallon pressure tank, starter, low water cutt-off, vacum gauge, pump and tubing, well seal and pressure gauge.

(2) The pump procured for installation in Well No. W-347 is stored in the Base Civil Engineer's office at Galena, except that the jet, twoopipes, low water cut-off, depth tube, and well seal are installed in the well. The pump set is as follows: Pump - Myers HCM-75, Serial No. 460-62, Motor - 3/4 HP Century Type CS Frame J56C EMI 8-101433-01, 60 cycle, single phase, $3450 \mathrm{rpm}, 115 / 230$ volts, complete with 80 gallon pressure tank, starter, 
low water cut-off control, depth and pressure gauges, pump, and all fittings.

(3) The pump procured for installation in Well No. W-346 is stored in the Base Civil Engineer's office at Galena, except that the jet, two-pipes, low water cut-off, depth tube and well seal are installed in the well. The pump set is as follows: Pump - Myers HP-50-D Serial No. 46062, Motor Wagner Electric, Type RK, 1/2 HP, single phase, 60 cycle, $3450 \mathrm{rpm}$, 115/230 volts, Model 48-59233-01 complete with 42 gallon pressure tank, starter, low water cut-off control, depth and pressure gauges, bicycle pump, and all fittings.

\section{CHEMISTRY OE GROUND WATER}

\section{a. Chemical Constituents}

The chemical quality of the ground water at Galena Airport has been the chief problem in developing a satisfactory water supply. The water from the aquifer at $200 \mathrm{ft}$ is high in iron, carbonate, and other deleterious substances, and the total amount of dissolved solids is high. Adding to the problem is contamination of the upper water layers by aviation fuels and oil. Iron may occur in a water sample in both dissolved form and as iron compounds in solid form. Iron in solution in groundwater is present chiefly as ferrous iron; but a smaller amount of iron occurs in the structure of complex organic substances in solution. Samples of water obtained from wells are often clear and appear to be good water. But upon.oxidation the ferric iron settles out, the samples become reddish brown in color and the taste is quite pungent. A chemical analysis of water from one of the CAA wells drilled in 1954 was made by $E$. L. Long, Testing

$$
14-\mathbf{a}
$$


Section, $\mathrm{ADE}$ on 2 March 1955 and showed $4.2 \mathrm{ppm}$ ferrous iron and $5.1 \mathrm{ppm}$ total iron for both an untreated water sample and a chlorinated water sample. Iron in solution as organic complexes is not detected by the analytical methods used for ferrous and ferric iron. Separate analyses made from time to time by W. M. Knoppe of ADE indicate that this organiccomplex iron present amounts to approximately $0.6 \mathrm{ppm}$.

Iron in solid form occurs as coatings of iron oxides around sand and silt grains.

The iron and total solids still remaining in solution after creatment has caused problems of scaling and corrosion in boilers and ateam pipes. Petroleum products may cause a scum to form inside boilers and pipes and would also contribute to scaling if present in significant quantities in the shallow ground water.

Chemical analyses of the water presently used are presented for Well No. 2, CAA Well No. 4, and the well at the Alert Building in Tables 2, 3, and 4 of the Appendix.

\section{b. Metkod of Analysis}

Tssting methods generally followed are those published in the 1961 ASTM standards, Volume 10. Samples as received contained both precipitated iron and fine-grained soil. In many instances traces of Iron precipitate were contained on the sample bottles. To overcome this condition a known portion of the sample was filtered. Total solids in solution were determined for the fiItrate by evaporation. The residue on the filter plus the total solids left after evaporation were dissolved in an excess of bydrocloric acid ( $\mathrm{KCl}$ ) and returned to the sample bringing the sample back 
to the original sample volume. Excess acid in this addition redissolved all available iron in the sample. The sample was again filtered to remove silt and total iron was determined on the filtrate.

\section{TEST RESULTS}

a. Quality of Water

Table 2 sumarizes chemical analyses of both the permanent wells now in use at Galena Airport and the shallow observation test wells drilled in this program. The permanent wells were sampled on various dates from 14 February 1963 to 11 April 1963.

Permasent Well No. 2 located near the Water Treatment Building is the main source of water supply at the present time. The well is probably $200 \mathrm{ft}$ deep. Three chemical analyses of this water, sampled at the well, show that total iron ranges from 4.6 to $7.6 \mathrm{ppm}$ and total solids from 314 to 454 ppsa.

The permanent well at the Alert Hangar, 210 feet in depth, shows $8.8 \mathrm{ppm}$ total iron and $461 \mathrm{ppm}$ total solids on the basis of one sample taken at the well.

CAA Well No. 4.in Building 400, Flight Service Station shows 24.8 ppm total iron and 864 ppm total solids.

Of the shallow observation wells drilled in the program, the data for Test Hole No. W-348 at the Water Treatment Bldg. are nost complete (Tables 2 and 5). Water samples from this well were taken at four different depths during six pumping tests. The data presented in Table 2 for this test well show that water containing only a trace of iron occurred in the uppermost layer at $32.5 \mathrm{ft}$, and thot deeper layers below a depth of approximately $35 \mathrm{ft}$ 
contained iron in higher concentrations, up to $41.2 \mathrm{ppm}$ total iron. A significant result of the pumping tests was the marked decrease in iron content with continued pumping at this test well. In each of four pumping tests the total iron content decreased to $1.0 \mathrm{ppm}$ or less after approximately 3 hours of pumping. The total solids content in these samples ranged from 580 to $960 \mathrm{ppm}$ and these values are considerably higher than the total solids in the deeper permanent well No. 2. In Test Well No. W-348 no significant variation in the amount of total solids was noted in water samples taken at various depths as drilling progressed, nor was any significant variation noted with the duration of pumping time.

Test Well No. W-347 was sampled at a depth of 35.5 ft. Five water samples were taken at various times during a 24-hour pumping test. The iron content of 14 ppm for samples from this well is over twice as high as water used at present, and no change in iron content was shown with duration of pumping. The total solids ranged from 474 to $532 \mathrm{ppm}$, only slightly higher than that in water from perasnent well No. 2 .

Test Well No. W-346 was sampled when the well had reached depths of 37,46 , and 56 feet. The total 1ron content at $37 \mathrm{ft}$ was 1.1 ppm; at 、 $4.6 \mathrm{ft}, 16.0 \mathrm{ppm}$; and at $56 \mathrm{ft}$, the Iron content ranged from 27.5 to $52.2 \mathrm{ppm}$. The increase in iron content with depth is similar to that which occurred in Test Well No. W-348. The total solids at $37 \mathrm{ft}$ were $824 \mathrm{ppm}$; at $46 \mathrm{ft}$, $1880 \mathrm{ppm}$; and at $56 \mathrm{ft}$, the concentration ranged from 976 to $1504 \mathrm{ppm}$. These values for total solids are about two to five times higher than that for water from permanent well No. 2 . 
Test Well No. W-345 was sampled when the well was 50 feet deep. Three water samples showed a total iron content of 1.6 to $3.6 \mathrm{ppm}$, and a total solids content of 1109 to 1192 ppm. These samples were obtained at various times during a 2 -hour pumping test and no significant change occurred in either iron content or total solids content, with duration of pumping. No evidence was obtained in the test drilling that the low-iron content is underlain by an impermeable stratum which would keep it separated from underlying water high in iron. It seems probable that the upper layer of low-iron-content water is the result of oxidation due to contact with astmospheric oxygen presert in the overlying strata.

\section{b. Quantity of Water}

The water consumption at Galena Airport for 1961 is estimated at $18,688,000$ gallons per year (Feulner, 1962). This quantity of water is equivalent to approximately 36 gallons per minute of continuous flow. Data from the test wells indicate that the yield to be expected from a well drawing only the uppermost low-iron-content water could be 2 to $5 \mathrm{gpm}$. The zone containing this low-iron-content water appears to be no thicker than $10 \mathrm{ft}$ at a maximum, as at Test Well No. W-345.

A tubular well, or a gallery well, constructed to a depth which would draw only the uppermost low-iron-content water would have difficulty maintaining both yield and quality of water because of seasonal fluctuations 
in the level of the water table. A hole (AH-4) drilled by the Corps of Engineers on 24 July 1953 at the Alert Hangar showed the static water level at an elevation of $103.5 \mathrm{ft}$. The water level in the permanent well at the Alert Hangar was at an elevation of $82.0 \mathrm{ft}$ on 25 November 1958 (P. R. Lord, U.S.G.S. intra-office Memo). Test Well No. W-347 of this program, also drilled at the Alert Hangar, showed the static water level on 1 April 1963 at an elevation of $91 \mathrm{ft}$. This comparison of water levels at different times of year would indicate a seasonal fluctuation of the water taple of at least $21 \mathrm{ft}$. Such a fluctuation in the water table would result in either a lack of sufficient water during the winter or low-water stage or the introduction of water high in iron during the sumer or high water state, depending on the depth of the well and its relation to the low-iron-content water zone.

\section{CONCLUSTONS}

The results of the chemical analyses of water samples from these four test wells can be sumarized as follows:

a. The total iron content increased with depths to values as much as seven times more than presently used water.

b. The total solids content is considerably higher than presently used water, and remains fairly constant with depth.

c. A thin uppermost layer of relatively iron-free water exists near Test Well Nos. $W-345, W-346$, and $W-348$.

A marked decrease in iron content occurred with time in several pumping tests made at Test Well No. $W-348$, but no decrease in Iron content was shown in a punping test at Test Well No. W-347. The chemical analysis 
for the shallow CAA Well No. 4 at the Flight Service Station, now in use, indicates an iron content about four times as high, and a total solids content about twice as high, as the water from permanent well No. 2 which is the present main source of water. This CAA well would appear to indicate that little, if any, lowering of iron content takes place with continued use.

The thin, uppermost layer of relatively iron-free water which occurs in three of the test wells would provide water lower in iron, but higher in total solids, than the water presently treated. From this standpoint, there does not, therefore, appear to be any advantage in the near surface water over the present Well No. 2 water. In addition, the large seasonal fluctuation in the water table would make practical utilization of the uppermost iron-free water difficult or impossible. Furthermore, a shallow water source such as this would be subject to contamination by sewage, aviation fuels, and oil. Such contamination appears likely to occur in the very near future. The treatment of such a shallow water to reduce the content of total solids and contaminants to a level conforming to health standards and practicability would probably be more complicated and expensive than the present water treatment facilities, and would produce water of little, if any, better quality. Based on a comparison of chemical analyses of treated and untreated water, (Tables 3 and 4) the water treatment facilities are not producing water meeting public health standards with respect to iron and total solids. For the treated water, the iron content of $1.70 \mathrm{ppm}$ is far above the maximum permissable level of $0.3 \mathrm{ppm}$ set by the U.S.Public Health Service. Although the carbonate hardness has been reduced by $100 \mathrm{ppm}$, the water is still hard. Whether or not the failure to produce water of better 
is due to inadequate treatment facilities, or to improper maintenance, is considered outside the scope of this report.

Utilization of shallow water would likely increase the scaling in boilers and pipes due to the higher content of total solids in the shallow water. In addition, the high probability of future contamination of shallow water by sewage, fuels, and oils would introduce additional problems of treatment.

The water obtained from permanent wells not presently tied in to the water treatment facilities can be improved by treatment.

$$
* * * * *
$$




\section{Literature Cited}

Eardley, A. J. (1938a), Unconsolidated sediments and topographic features of the Lower Yukon Valley: Geol. Soc. Amer., Bu11. Vo1. 49, p. 303-341.

(1938b), Yukon channel shifting: Geol. Soc. Amer., Bu11. Vo1. 49, p. 343-357.

Feulner, Alvin J. (1962), Water sources used by the United States Air Force in Alaska, 1960-61: A supplemental report, prepared by Ground Water Branch, U.S.G.S. for Alaskan Air Command, Anchorage, Alaska.

Pewe, Troy L. (1948), Terrain and permafrost of the Galena Air Base, Galena, Alaska: Permafrost Program Progress Report No. 7, Engineer Intelligence Division, OCE.

Smith, L. W. (1954), Location and conditions of water wells at Galena AFAF: Disposition Form in files of F\&M Branch, $\mathrm{ADE}$, Anchorage, Alaska. 
Table I. . . . . . . Climatological Sumary.

Table II ........ Chemical Analyses of Water.

Table III . . ... . . Chemical Analysis, Permanent Well No. 2, treated water.

Table IV . . . . . . Chemical Analysis, Permanent Well No. 2, untreated water.

Table V......... Chemical Analysis, Test Well No. W-348.

Fig. 1. . . . . . . Pump Attachment Diagram, Test Well No. $\mathrm{W}-346$.

Fig. 2. . . . . . . . Location Map of Test Wells and Permanent We118.

Fig. 3a........... Log of Test Well No. W-345. 3b. . . . . . . Log of Test Well No. W-346. 3c. . . . . . . . Log of Test Well No. W-347. 3d. . . . . . . Log of Test Well No. W-348. 
CLILA TO LO GI CAL SUMMARY

GALENA

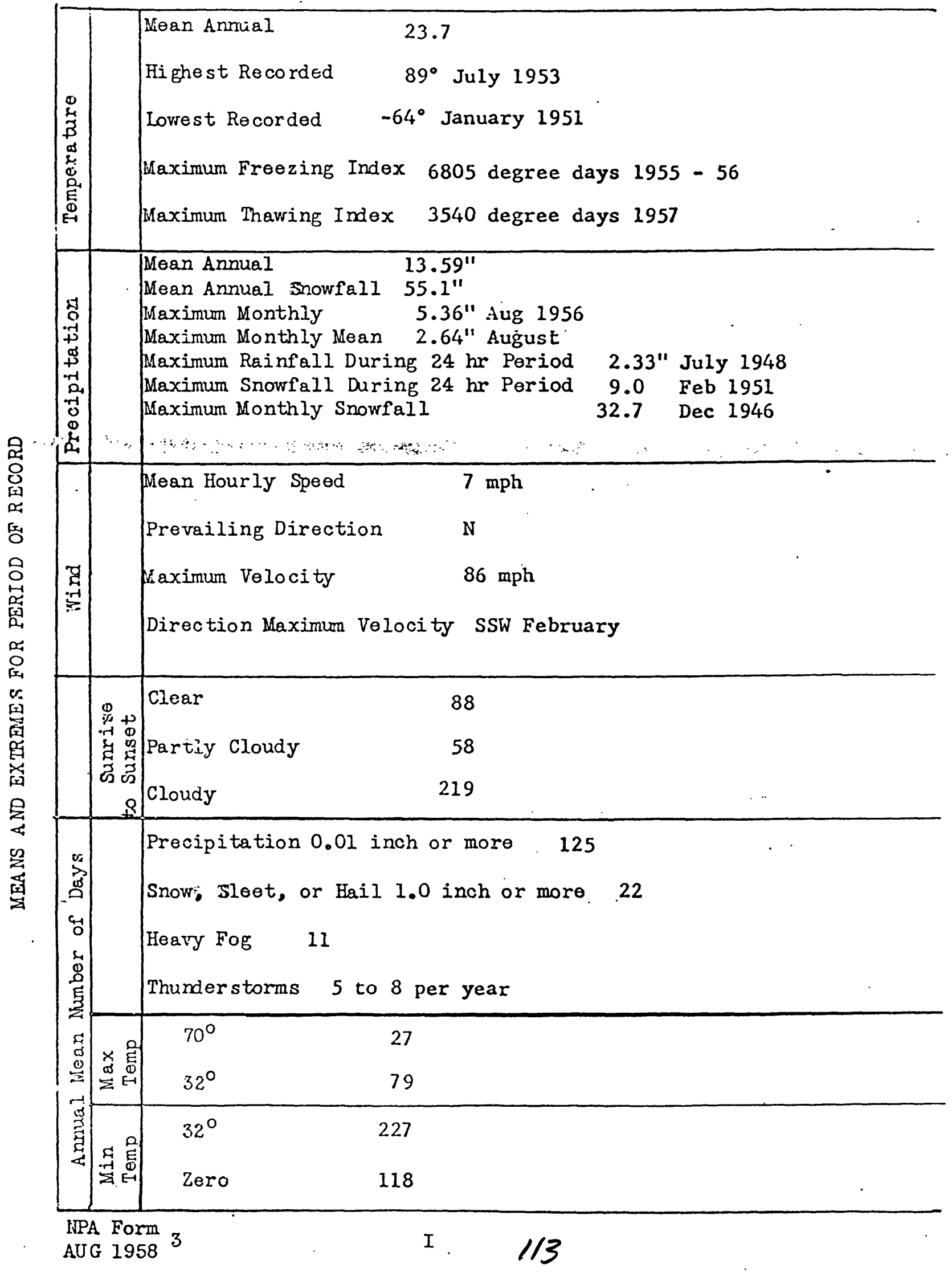


TABLE 2

Chemical Analyses of Water from Galena Airport

Results in $\mathrm{ppm}$, analysis by F\&M Branch, Test Lab

\begin{tabular}{lllll}
$\begin{array}{l}\text { Sample } \\
\text { No. }\end{array}$ & $\begin{array}{l}\text { Date } \\
\text { Sampled }\end{array}$ & pH & $\begin{array}{l}\text { Total } \\
\text { Solids }\end{array}$ & $\begin{array}{l}\text { Total } \\
\text { Iron }\end{array}$ \\
\hline
\end{tabular}

CAA WELL NO. 4 - B1dg 400, F1ight Service Station
1
14 Feb 63
$6.8 \quad 864$
24.8
$38-43$

PERMANENT WELL NO. 2 - BIdg 1578 - Sampled before treatment but after storage

$\begin{array}{lrlllll}2 & 14 \mathrm{Feb} 63 & 6.8 & 454 & 4.8 & 180-200 \\ 3 & 8 \mathrm{Apr} 63 & & 314 & 7.6 & 180-200 \\ 4 & 11 \mathrm{Apr} 63 & & 343 & 4.6 * & 180-200 \\ 4 \mathrm{~A} & 24 \mathrm{Apr} 63 & 7.0 & 298 & 5.2 & 180-200\end{array}$

PERMANENT WELL - Bldg 1428, Alert Bldg, untreated water except for chlorination
5
14 Feb 63
$7.0 \quad 461$
8.8
$190-200$

TEST WELL NO. W-345 - Ammo Storage Bldg, raw water

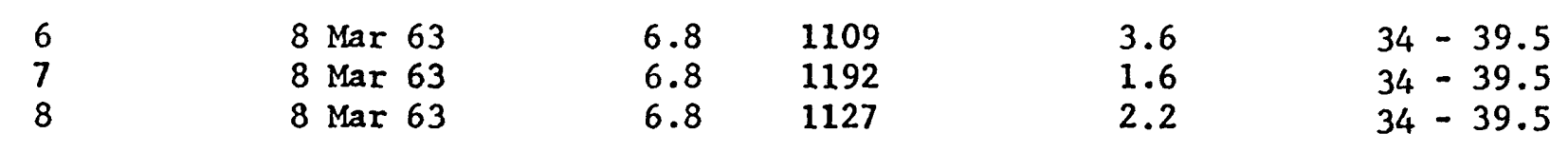

TEST WELL NO. W-346 - Guardhouse, raw water

$\begin{array}{rrrrr}9 & 14 \operatorname{Mar} 63 & 824 & 1.1 & 28-29 \\ 10 & 15 \operatorname{Mar} 63 & 1880 & 16.0 & 37 \\ 11 & 16 \operatorname{Mar} 63 & 1236 & 44.0 & 51.3-54 \\ 12 & 18 \operatorname{Mar} 63 & 1504 & 27.5 & 51.3-54 \\ 13 & 19 \operatorname{Mar} 63 & 976 & 52.2 & 51.3-54 ; \\ 14 & 19 \operatorname{Mar} 63 & 1224 & 33.5 & 51.3-54:\end{array}$

TEST WELL NO. W-347 - Alert Hangar - raw water

$\begin{array}{lllll}15 & 1 \text { Apr } 63-0830 \text { hrs } & 486 & 14 & 30-35 \\ 16 & 1 \text { Apr } 63-1400 \text { hrs } & 500 & 14 & 30-35 \\ 13 & 1 \text { Apr } 63-2000 \text { hrs } & 532 & 13.9 & 30-35 \\ 18 & 2 \text { Apr } 63-0400 \text { hrs } & 474 & 14 & 30-35 \\ 19 & 2 \text { Apr } 63-1115 \text { hrs } & 522 & 14 & 30-35\end{array}$




\begin{tabular}{|c|c|c|c|c|c|}
\hline Sample & $\begin{array}{l}\text { Date } \\
\text { Sampled }\end{array}$ & $\mathrm{pH}$ & Total & $\begin{array}{l}\text { Total } \\
\text { Iron }\end{array}$ & $\begin{array}{l}\text { Depth to Aquifer } \\
\text { When Samipled (Ft) }\end{array}$ \\
\hline
\end{tabular}

TEST WELL NO. W-348 - Water Treatment B1dg, raw water

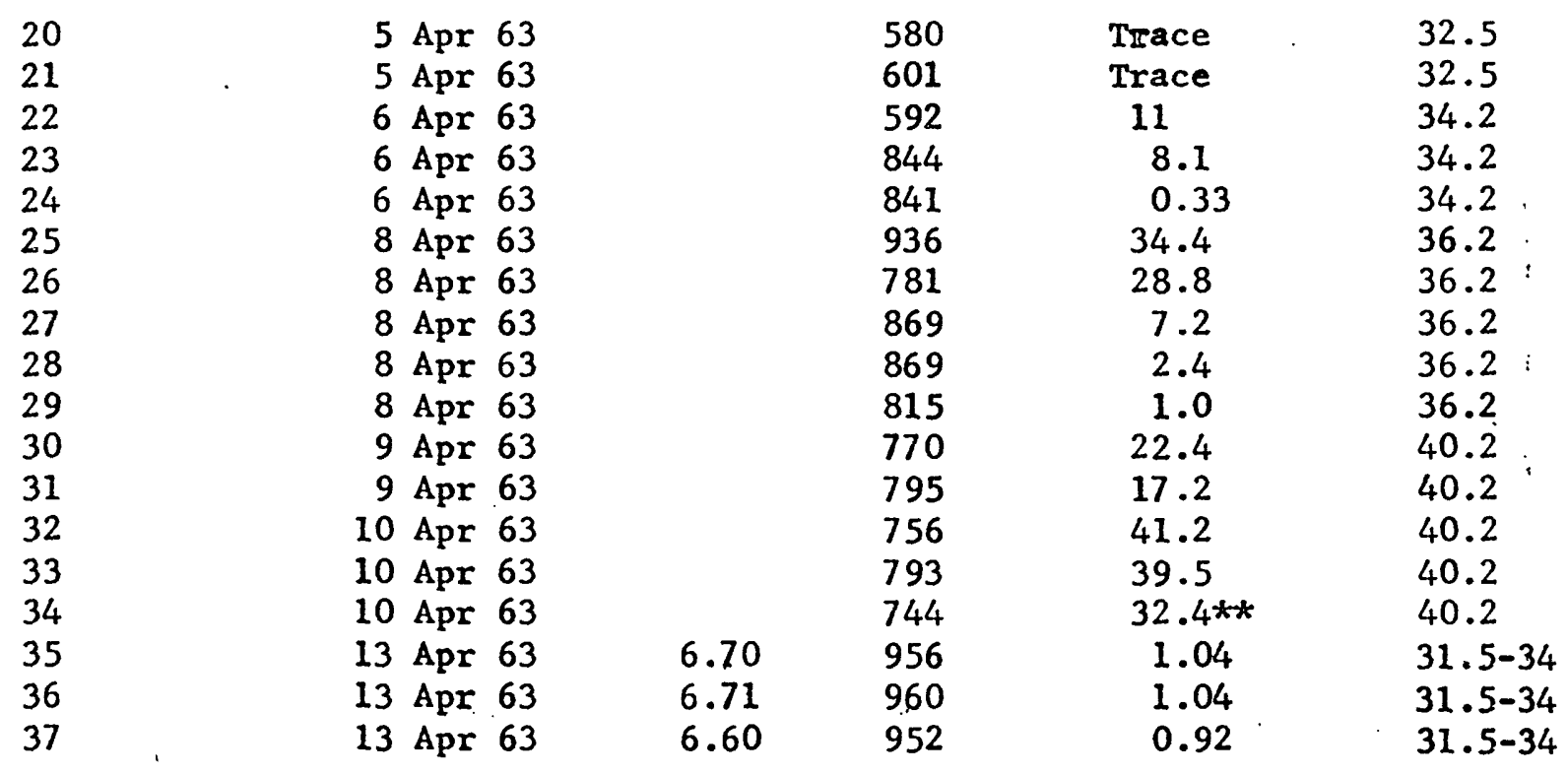

* Total $\mathrm{R}_{2} \mathrm{O}_{3}$ as $\mathrm{Fe}$ is $130 \mathrm{ppm}$.

** Total $\mathrm{R}_{2} \mathrm{O}_{3}$ as $\mathrm{Fe}$ is $199.9 \mathrm{ppm}$. 


\section{Table 3}

REPORT ON WATER

FOR

CONTRACT NO. DA-

DE IAB FILE NO.

\section{SOURCE: Galcne ATS}

SAMPLE \& LABEL: Permanent iell ịo. 2, treatcd ratier; Sanpled 15 ifarch 1963. Data from District C:e $i \in t$, USGS, Palmer.
REPORT DATE:

SUBMITTERS SAMPLE 10.

ó íay 1963

\section{REQUEST: Comple :e Ua er Anal":sis}

TEST RESULTS;

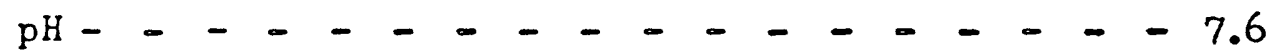

Conductivity - - - - - - - - - - - 452 mmhos

Total solids - - - - - - - - - - -

Calcium, as $\mathrm{Ca}--\infty-\infty-{ }_{-}-{ }_{-}-{ }_{-} \mathrm{ppm}$

Magnezium as $\mathrm{Mg}--,--,--,--$

Potassium

Sodium \&c:Potasalum, as Na $-\cdots,-\ldots$

Total Iron, as Fo,,,$-------\infty$

$1.70 \mathrm{ppm}$

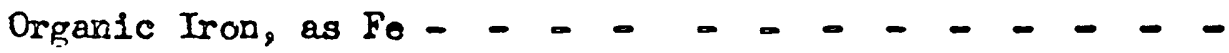

ppm

Manganese, as Mn,,,,---------

ppm

Silica; as $\mathrm{SHO}_{2}--,--,--,--,-$

$35 \mathrm{ppm}$

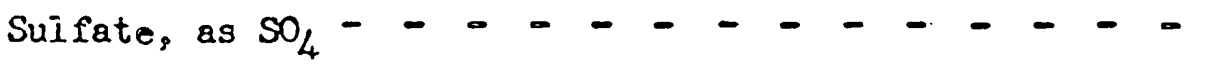

$1.0 \mathrm{ppm}$

Chlorido, as Cl - - - - - - - - - - -

5.0 ppm

Nitrate, as $\mathrm{NO}_{3}-------,----$

Fluoricie

$0.4 \mathrm{ppm}$

0.2

ppm

Alkalinity, Phenolphthalein, as $\mathrm{CaCO}_{3}--$

ppm

Total hardness, as $\mathrm{CaCO}_{3}--,------$

ppm

Carbonate hardness, as $\mathrm{CaCO}_{3}-{ }_{-}-{ }_{-}-{ }_{-}-$

ppm

Non-Carbonate hardness, as $\mathrm{CaCO}_{3}-{ }_{-}-{ }_{-}-{ }_{-}$

ppm

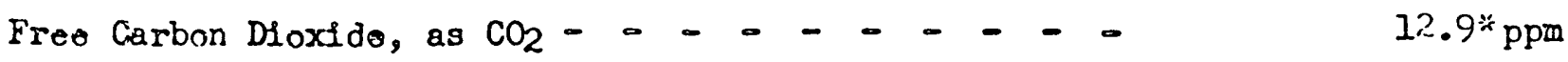

Froe Oxygun, as $\mathrm{O}_{2}-\infty-296$ ppm

$\mathrm{Ha} \mathrm{ICO}_{3}, \mathrm{a}=\mathrm{ICO}_{3}$

REMARKS: $\mathrm{Na}_{2} \mathrm{CO}_{3}$ as $\mathrm{CO}_{3}$ * Calcula ied 
REPCRT ON WATER

FOR

CONTRACT NO. RA-

DE IAB FILE NO.

\section{Table 4}

REPORT DATE: 7 l'OY 20,63

SUBMITTERS SAMPLE NO.

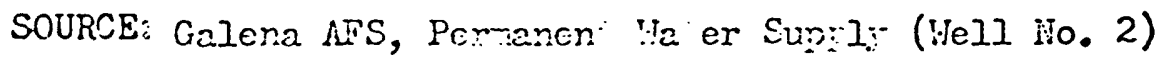

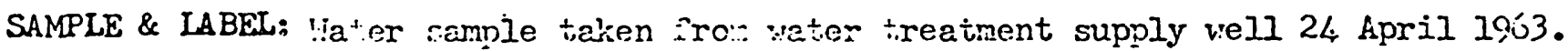
REQUEST: Complete Ind. Chem. analr"sis

TEST RESULTS:

$\mathrm{pH}-------------7.0$

Conductivity - at $23^{\circ} \mathrm{C}-\ldots-\ldots$

417 mmhos

Total solfds $-\infty--,--,--,--$

$208 \mathrm{ppm}$

Calcium, as $\mathrm{Ca}-{ }_{-}-{ }_{-}-{ }_{-}-{ }_{-}-$

$84 \quad \mathrm{ppm}$

Magneslum, as $\mathrm{Mg}-{ }_{-}-{ }_{-}-{ }_{-}-{ }_{-}-$

$14 \mathrm{ppm}$

Sodium \& Potassium, as Na $-{ }_{-}-{ }_{-}-{ }_{-}-$

7 ppm

Total Iron, as Fo - I $^{-}-{ }_{-}-{ }_{-}-{ }_{-}$

$5.2 \mathrm{ppm}$

Organic Iron, as Fe $-{ }_{-}-{ }_{-}-{ }_{-}-{ }_{-}$

Trace ppm

Manganese, as Mn - - - - - - - - - - -

$0.0 \mathrm{ppm}$

Silica; as $\mathrm{SiO}_{2}--,--,---,--$

35 ppm

Suifate, as $\mathrm{SO}_{4}--\infty-\infty-+---{ }_{-}-$

$6 \mathrm{ppm}$

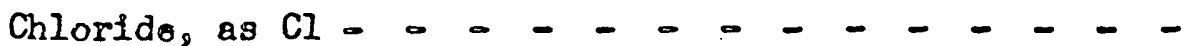

4.4 ppm

Nitrate, as $\mathrm{NO}_{3}--\infty--------$

0.6 ppm

Alkalinity, Methyl Orango, as $\mathrm{CaCO}_{3}-{ }_{-}-{ }_{-}-{ }_{-}$

245 ppm

Alkalinity, Phenolphthaleln, as $\mathrm{CaCO}_{3}-\mathrm{C}^{-}-\mathrm{-}^{-}-$

0.0 ppm

Total hardness, as $\mathrm{CaCO}_{3}-$ - - - - - - - -

245 ppm

Carbonate hardness, as $\mathrm{CaCO}_{3}-{ }_{-}-{ }_{-}-{ }_{-}-$

$245 \mathrm{ppm}$

Non-Carbonate hardness, as $\mathrm{CaCO}_{3}-0$ - - - -

$0.0 \mathrm{ppm}$

Freo Carbon Dioxido, as $\mathrm{CO}_{2}$ - o Field Relizole Only - -

ppm

Frec 0xygen, as $\mathrm{O}_{2}=-0$-Field Reliajle orly - -

ppm

REMARKS: 


\section{Table 5}

REPORT ON WATER

FOR

CONTRACT NO. DA-

DE IAB FILE NO.
REPORT DATE:

SUBMITTERS SAMPLE NO. 23

SOURCE: Galena APS, Fest :eil I:o. W-348

SAMPLE \& LABEL: Sarmled 13 Anril 1963, 3: hr: of pumping after setting screen. Depth 34'

REQUEST: Complete Jater Anal:-sis

TEST RESULTS:

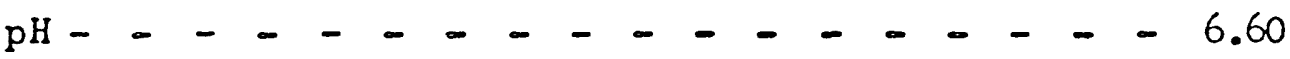

Conductivity $-\infty--,-,-,-$

1176 mmhos

Total solids $-\ldots+-\infty-\infty-$

$952 \mathrm{ppm}$

Calcium, as $\mathrm{Ca}$

$256 \mathrm{ppm}$

Magneslum, as Mg - - - - - - - - - -

$30 \mathrm{ppm}$

Sodium \& Potassium, as $\mathrm{Na}-{ }_{-}-{ }_{-}-{ }_{-}-$

$8 \quad \mathrm{ppm}$

Total Iron, as Fo - - - - - - - - - -

$0.92 \mathrm{ppm}$

Organic Iron, as $\mathrm{Fe}$

Trace ppm

Manganese, as Mn - N $_{-}-{ }_{-}-{ }_{-}-{ }_{-}-$

ppm

Sillca, as $\mathrm{SiO}_{2}-\infty,--\infty-\infty-\infty-\infty$

$9.6 \mathrm{ppm}$

Suifate, as $\mathrm{SO}_{4}-----------$

$11.0 \mathrm{ppm}$

Chlorido, as Cl

$4.4 \quad \mathrm{ppm}$

Nitrate, as $\mathrm{NO}_{3}-\infty-\infty-\infty-\infty-\infty$

$0.8 \mathrm{ppm}$

Alkailnity, Methyl Orange, as $\mathrm{CaCO}_{3}-{ }_{-}---$

455 ppm

Alkalinity, Phenolphthaloin, as $\mathrm{CaCO}_{3}-{ }_{-}-{ }_{-}-$

0.0 ppm

Total hardness, as $\mathrm{CaCO}_{3}-\infty-\infty-\infty-$

Carbonate hardness, as $\mathrm{CaCO}_{3}-{ }_{-}-{ }_{-}-{ }_{-}-$

Non-Carbonate hardness, as $\mathrm{CaCO}_{3}-\cdots-$

Freo Carbon Dloxido, as $\mathrm{CO}_{2}=-\ldots-\ldots$

ppm

739

ppm

454

ppm

ppm

Free Oxygon, as $\mathrm{O}_{2}-\infty-\infty-\infty-\infty-$ ppm

REMARKS:

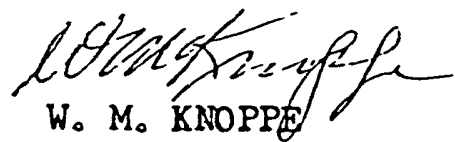




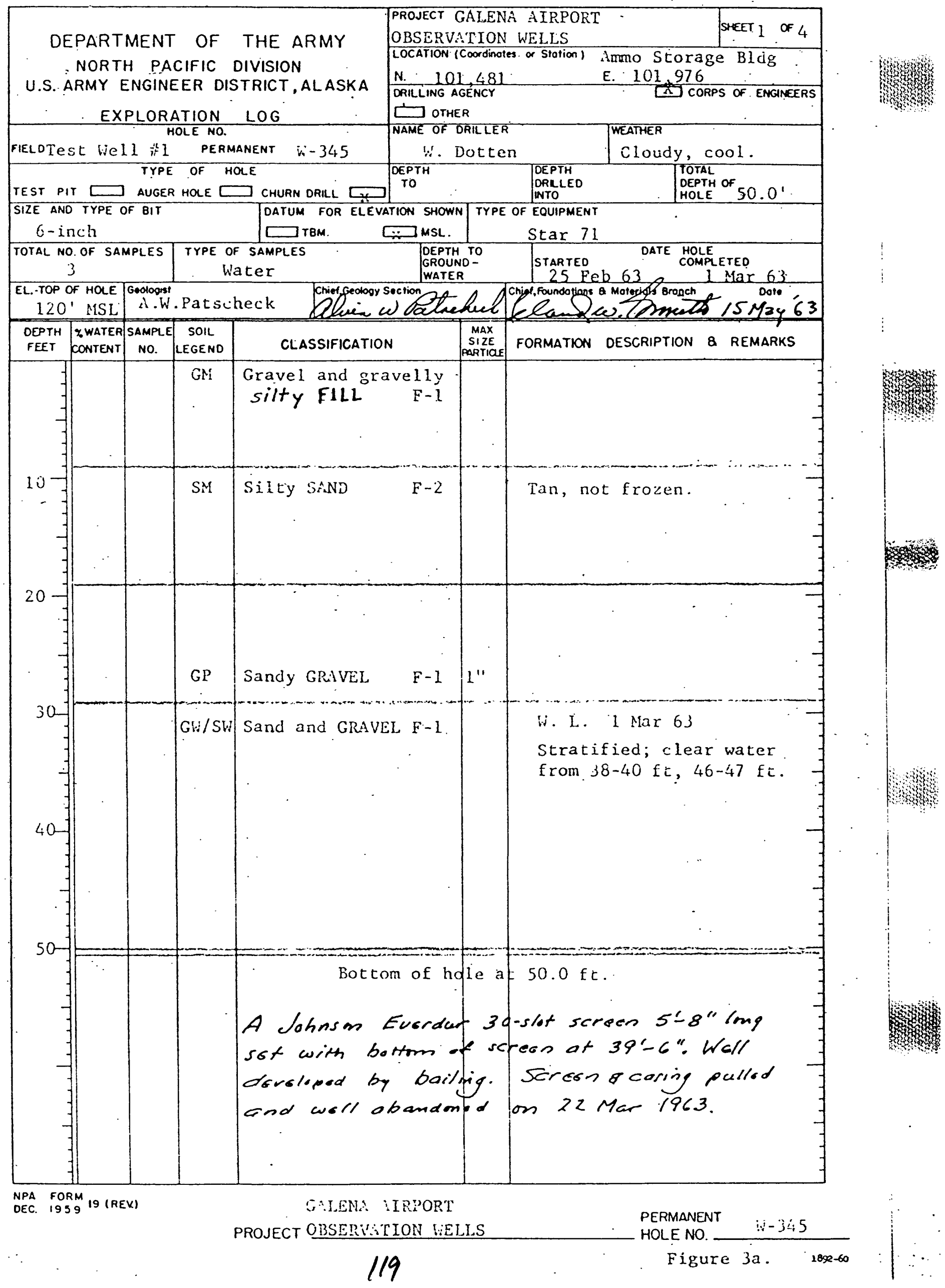




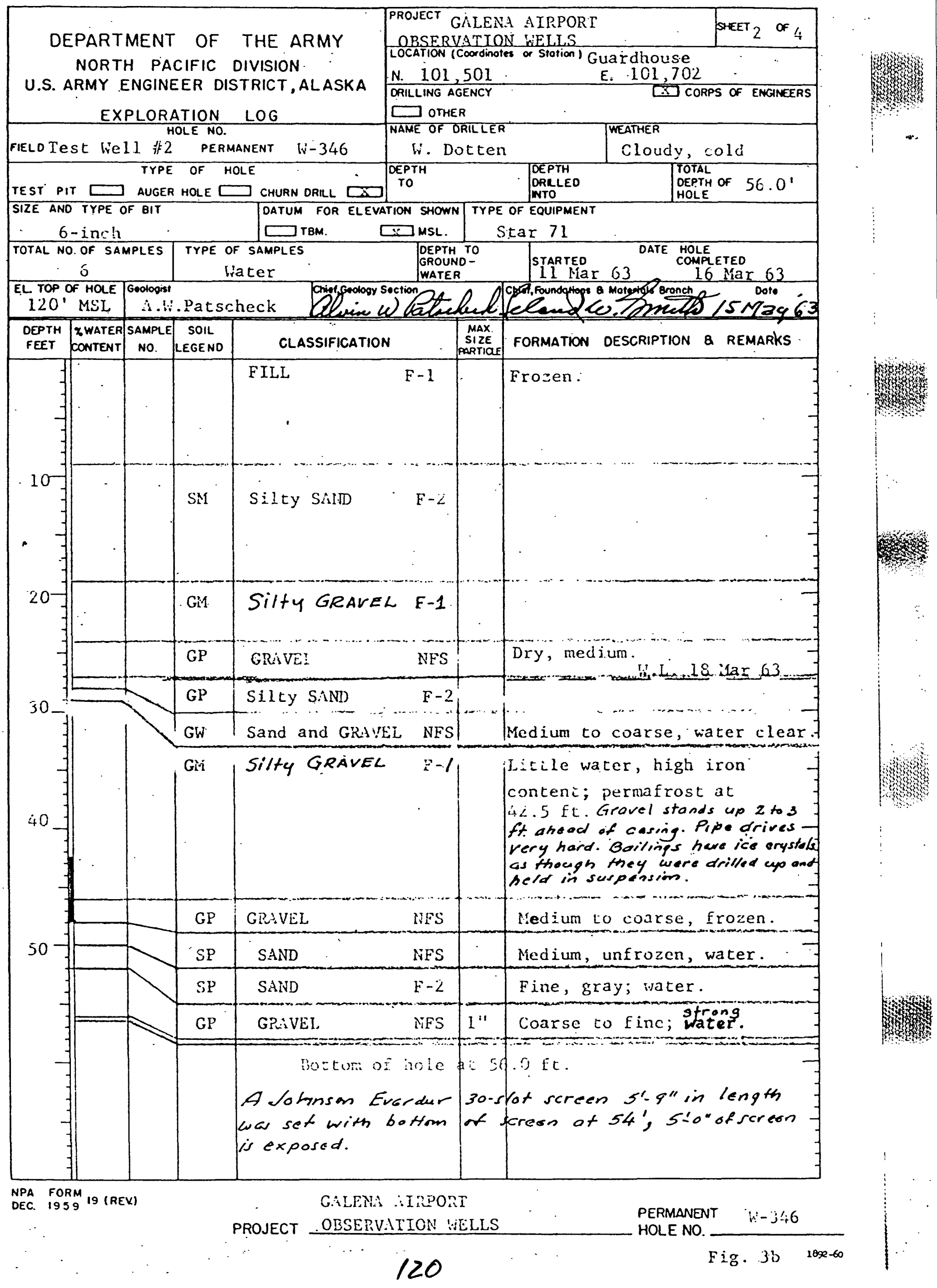




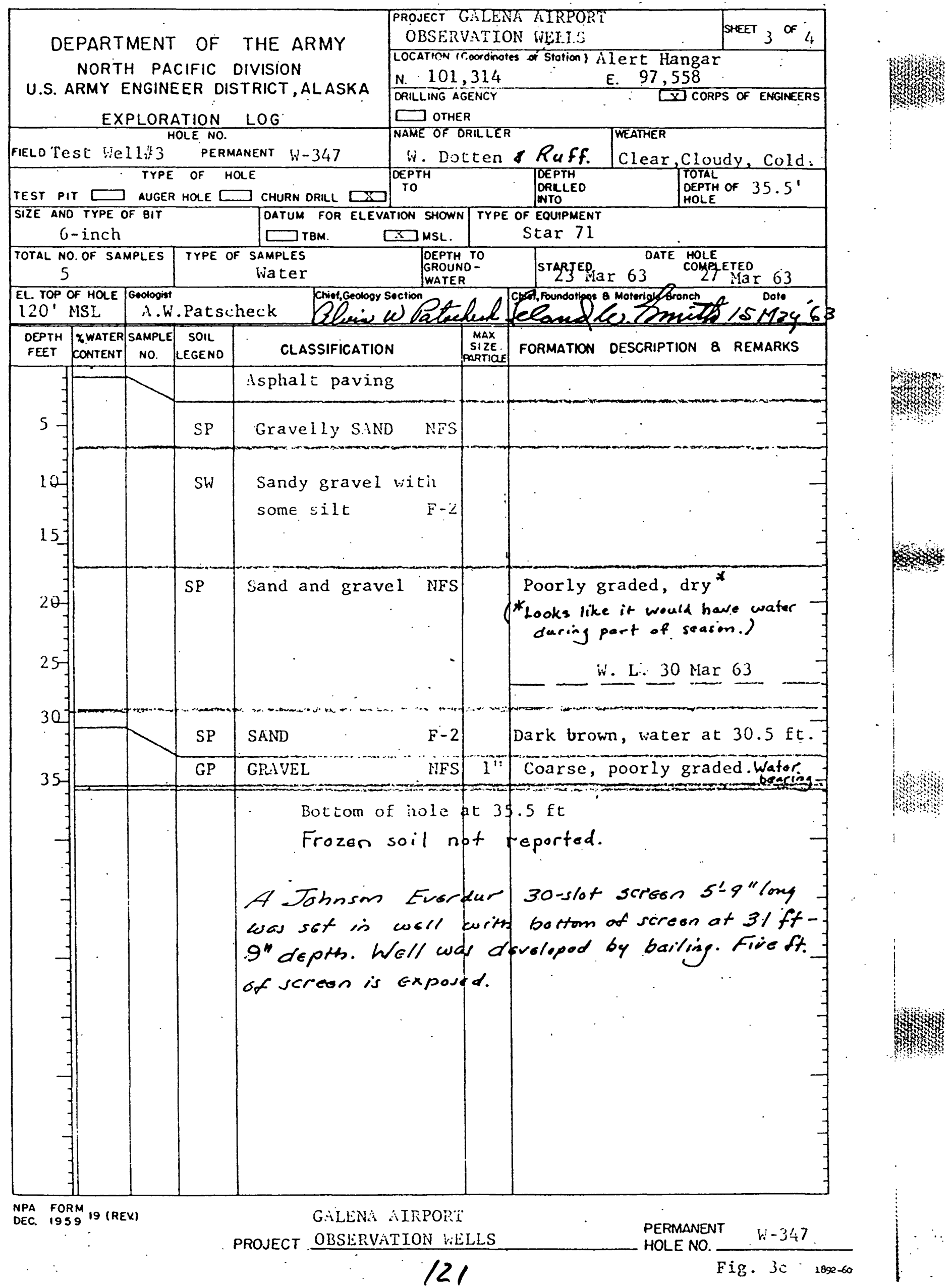




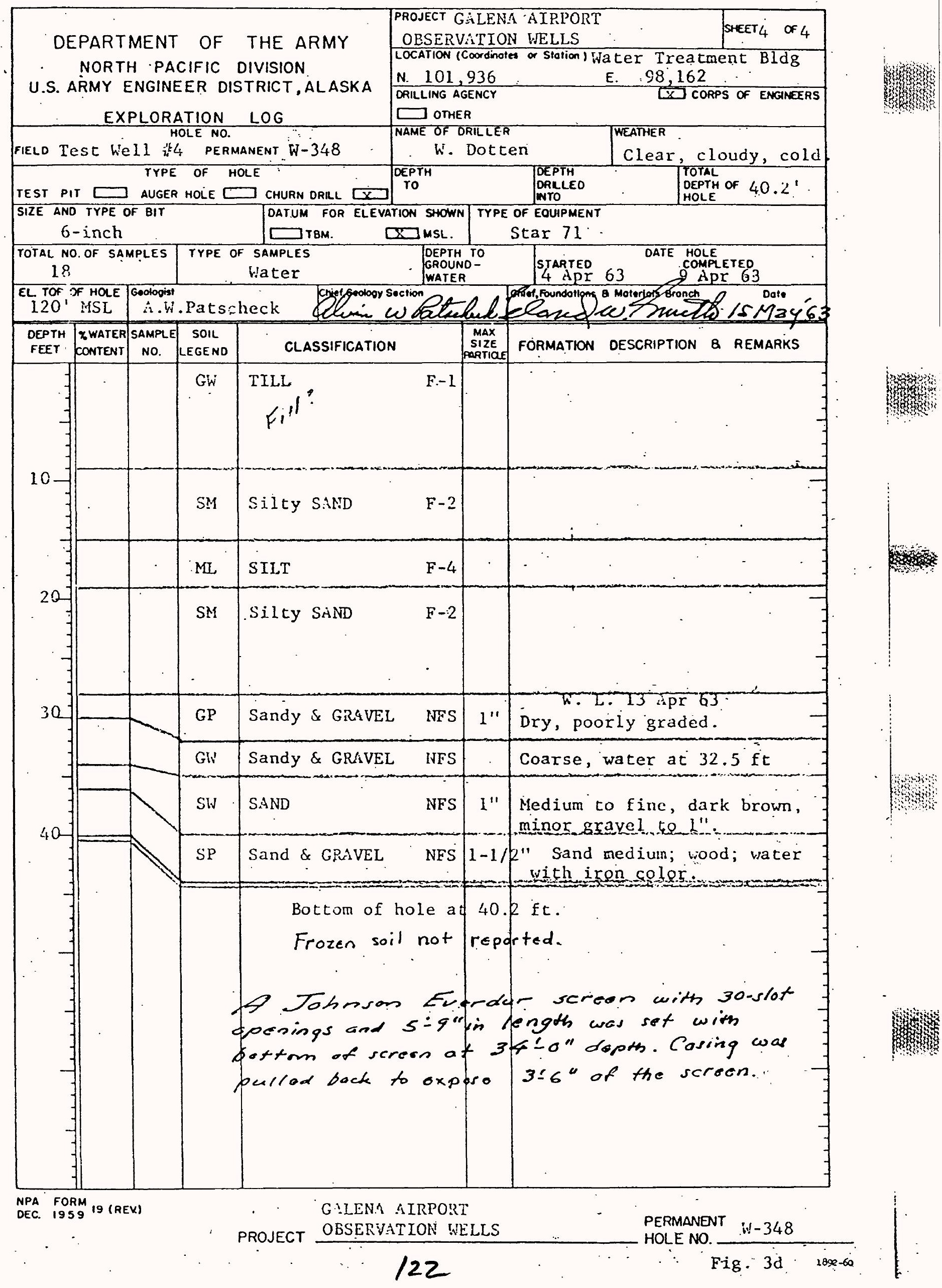




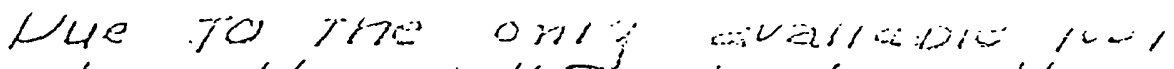

which show the well niado less than six(6) gipm

I feel hooking the purve into the existing twe al prosure tarit un wh $(5 \mathrm{~s} \%$

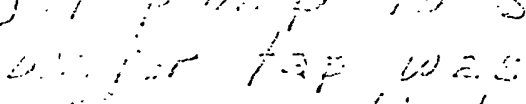

Whe would bo

were bahanced, wowh elin and the necd of

the large $4.5 \times 6.5$ tan storige tante

Thing these factors into considention, Ifeet a hole shouta be made thres the conerote wall, hily enowh to allow the horizontal giocing from well teal to pump to be on a slipht incline. by fabricativa suitable support for the purre. The dischatege could go direatly intothargenestitornge fank with a float controll to sich and stort tho pumip.

Stooul Note:

A. D. Ruefe $\left.\right|_{\text {Concelo wall }}$

Insfall chlorinator in

Floot switith line at" this paint as water may bo, or bocoms, polvetod.

Existing Fis

42 gal

Pitus Turn

$1 \times \operatorname{sing} P$ Pimp

is

ACUP FOR

Nuto: Sotpunp for onougin furnition bos $A$ Q

EVIVELL a PUMP AT awray frem wall to and frowing in wintor.

Con Suris

IARO HOUSE WELL-GALENS

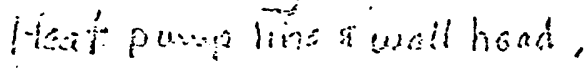
VII

123

$1 \operatorname{ray}^{\prime} 63 \mathrm{Ial}$ Flaure 1 
SUPPLEIENT NO. 1 TO REPORT OF FOUNDATIONS AND MATERIÁLS BRANCH GIILENA AIRPORT OBSERVITION WELLS ĠLENA, ALASKA

Prepared by

Foundations and Materials Branch

25 June 1963 
1. Scope . . . . . . . . . . . . . . . . 1

2. General . . . . . . . . . . . . . 1

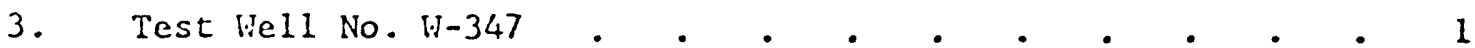

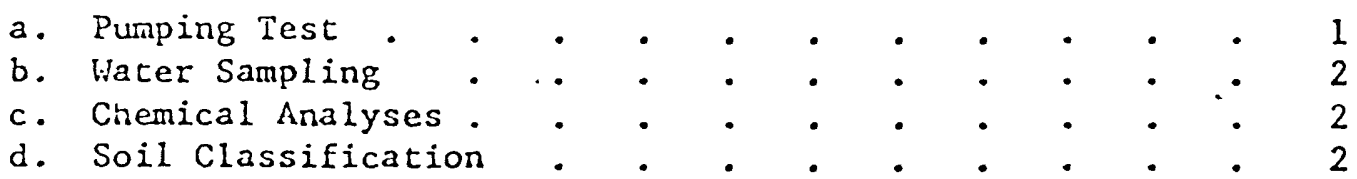

4. Test Hell No. W-348 . . . . . . . . . 3

a. Pumping Test. . . . . . . . . . . 3

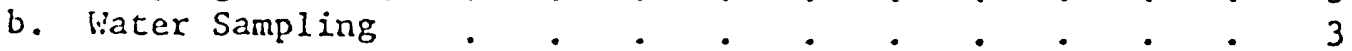

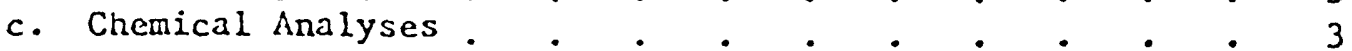

d. Soil Classification $\quad \cdot \quad \cdot \quad \cdot \quad \cdot \quad \cdot \quad \cdot \quad \cdot \quad \cdot \quad \cdot \quad \cdot \quad \cdot \quad \cdot \quad 3$

5. Test Well No. W-345 . . . . . . . . . . . 4

6. Test Well No. W-346. . . . . . . . . . . 4

7. Permanent Water Well No. 1 . . . . . . . . . 4

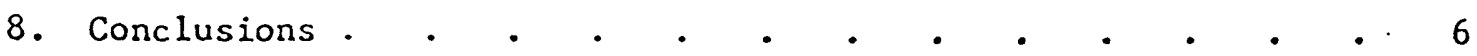

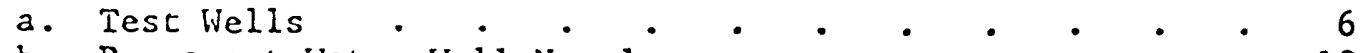

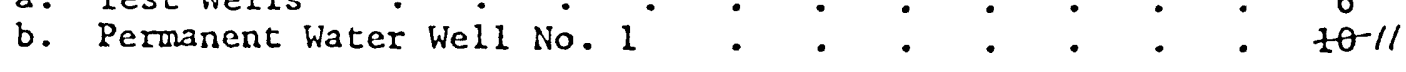




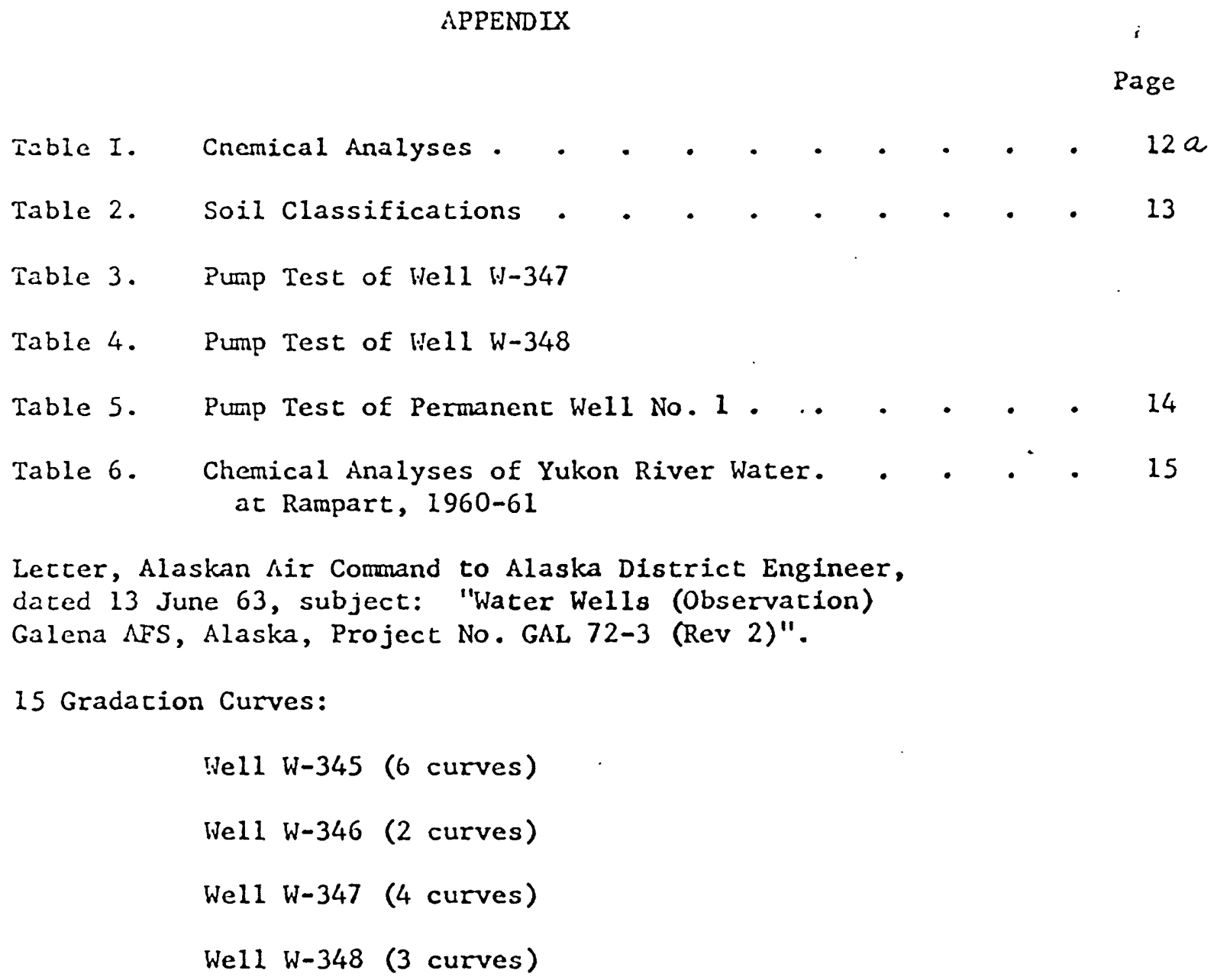


SUPPLENENT NO. 1 TO REPORT OF

FOURDATIONS $\therefore \mathrm{ND}$ ULITERIALS BRINCH

G/LENA $\triangle$ IRPORT OBSERVATION WELLS

GALENA, ALISKA

1. SCOPE: This report, which is a supplement to the report on the Galena Airport Observation Wells dated 10 May 1963, covers pumping tests, water sampling, chemical analyses, gradation curves, and interpretations of data at Test Well W-347 (adjacent to the Mlert Hangar) and Test Well W-348 (adjacent to the Water Treatment Building). Also reported are results of a pumping test on Permanent Water Well No. 1.

2. GENERAL: The basic report dated 10 May 1963 discusses the geology, groundwater, and general development of the area; locations of the test water wells and permanent vells are given in this report together with the results of earlier chemical analyses of water from the test wells. Pumping tests described in the present report were made with a Fairbanks Morse 1/2 HP, 115 volt, $3450 \mathrm{RPM}$ submersible pump only on Test Well No. 348 . A jet pump was used on Test Well No. $W-347$, and the presently installed Jacuzzi jet pump was used on Permanent Well No. 1. Soils have been classified on the basis of mechanical analysis of samples; the gradation curves accompany this report.

3. TEST WELL NO. $\mathrm{H}-347$

\section{a. Pumping Test}

Well No. W-347 adjacent to the Alert Hangar was test pumped on 3 June 1963 from 1010 to 1610 hours. The static water level at the start of pumping was at $1.1 \mathrm{ft}$ depth (elevation $118.9 \mathrm{ft}$ ), measured from the top 
of the aspiralt pavement which exists at the sitc (elevation of pavement 120.0 Et). The vell was pumped at the rate of 12.4 gallons per minute for six hours. The drawdiown level, measured from the top of the asphalt pavement, was at $2.58 \mathrm{ft}$ depth at 1010 , and at $2.83 \mathrm{ft}$ depth at 1610 . A small amount of fine sand was present in the water pumped during the test. The data on this pump test are given in Table 3 .

b. Water Sampling

The first sample of well water was taken at the start of the pump test at 1010,3 June 1963. A second sample was taken at 1310 hours and a third and final sample at 1610 hours, just before the pump test was concluded.

\section{c. Chemical finalyses}

Chemical analysis of the three water samples mentioned above consisted of determination of the total sollds and the total iron (as $\mathrm{Fe}$ ). Results of the analysis are given in Table 1 .

Total iron decreased during pumping from $28.5 \mathrm{ppm}$ to $8.0 \mathrm{ppm}$. A smaller percentage decrease occurred in total solids, from $606 \mathrm{ppm}$ at the start of the pump test to $500 \mathrm{ppm}$ at the conclusion.

\section{d. Soil Classification}

The results of mechanical analysis of four soil samples obtained during drilling from Well No. W-347 are show graphically in the gradation curves in the Appendix of this report. The results of these tests are summarized in Table 2. 


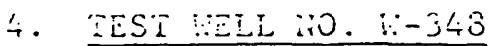

\section{Pumins Tese}

IEl: No. ii-340 adjacent to the liater Treatment Building was test pumped on 31 Yay 1963 from 0920 to 1640 hours. The static rater level at Lis scürt of pumping vas 2.33 íc depch (clevation 117.67 Et), measured from the ground surface (elevation $120.0 \mathrm{ft}$ ). The viell was pumped at the rate of 20.2 galions per minute for 7 hours 40 minutes. The drat:dorm level, measured Erom the ground surface, vis at $8.4^{\circ}$ ft depth at 0920 , and at $8.73 \mathrm{ft}$ depth at 1640, just before concluding the pumping test. A small amount of sand was present in the vater pumped during the test. The data on this pump test are given in Table 4.

\section{b. Vater Samoiing}

The first sample of rell vater ns taken at the start of the pump test at 0920, 31 May 63. is seconci sampie ras taken at 1240 hours, and a third and final samile at $\$ 520$ hours.

\section{c. Chenice: Analyses}

Chemical analysis of the three water samples mentioned above were made to cetermine total soiids and total iron (as Fe). Results of the analysis are given in Table 1.

Total iron decreased during pumping from 64 ?pm to $0.35 \mathrm{ppm}$. $\therefore$ smaller percentage decrease occurred in total soiids, from 1664 ppm at the start of the tese to $1320 \mathrm{ppm}$ at the conclusion.

\section{d. Soil Classification}

The results of mechanical analysis of three soi? samples obtained during driling from Vell $\mathrm{b}-348$ are shown graphically in the gradation curves 
in the ippendix of the report. The results of these tests are sumarized in Table 2 .

\section{TEST UELL $\quad 1-345$}

Gradation curves for six soil samples obtained during drilling from tinis well are given in the Appendix. The results of these tests are sumarized in Table 2.

\section{TEST IVELL $\mathrm{W}-346$}

Gradation curves for two soil samples obtained during drilling from this well are given in the Appendix. The results of these tests are sumarized in Table 2.

7. PERMANENT WATER WELL NO. 1

a. General: Well No. 1 is located in an addition to the east end of the Fire Station, Galena Airport. In 1954 this well was the primary source of water for the base. The well is $210 \mathrm{ft}$ deep and is cased with 6 inch standard steel casing. Whethery or noty a well screen was installed is not definitely known, but, according to construction drawing file 157-F-N dated 9 September 1944 of the Federal Aeronautics Administration, a perforated screen was to be installed. The pump now installed in the well is a Jacuzzi Jet Pump, type 75-T-44M, Serial No. 50290, with 60 feet of 4 inch jet pipe column, 40 feet of $2-1 / 2$ inch jet pipe column above the jet and 22 feet of 2 inch jet pipe tail below the jet. The pump is driven by a $7-1 / 2 \mathrm{HP} 1800$ rpm, 3 phase U.S.Electric motor, Serial 355426. This pump is mounted on an open steel frame 17 inches high by 17 inches square, and was installed a considerable time before 1951. The top of the casing is 18 inches below 
the pump house floor level. The discharge line is 2 inch in diameter. In 1954 this set up produced between 12,000 and 15,000 gallons per day with a static water level of $19.6 \mathrm{ft}$ and a pumping water level of $20 \mathrm{feet} \pm$, or a drawdown of less than one foot. The well has been unused for several years; meanwhile the pump room has been used as a Fireman's office.

b. Pumping Test - Permanent Well No. I was pump tested on 7 - 10 June 63, using the Jacuzzi jet pump installed on the well head. It was necessary for the Base electrician to run in 3-phase AC power in order to energize the pump motor. The test was begun at 1000 hours 7 June and concluded 0800 hours 10 June. The data on this test are given in Table 5. The discharge was clear soon after the initial start up of the test, and no sand was reported. Static water level at the beginning of the test was $5.25 \mathrm{ft}$ below the top of casing. Drawdown to initial pumping level was 2.0 feet and to the pumping level at 1600 hours 8 June was $3.08 \mathrm{ft}$. A recovery rate was not run in view of the obvious competence of the well at the 46 gpm pumping rate. This pump test demonstrated this well is capable of continuously producing $46 \mathrm{gpm}$ or 66,240 gallons per day.

c. Water Sampling - The first chemical sample of pumped water was taken by Mr. Alfred Ruff, Alaska District Engineer Driller, 7 June 63, 1000 hours, at the start of the test; the second, 8 June at 1000 hours; and the final, 8 June at 1600 hours. The first biological sample of the pumped water was taken 7 June 63, 1000 hours, at the start of the cest by Sgt. Dennis, Base Medical Technician; the second 7 June at 1400 hours, and the final 8 June at 1000 hours. 


\section{d. Analysis of fater Samples:}

(1) Cncmical Tests - Cnemical analysis of three water samples, caken by Mr. Ruff, was performed by the Alaska District Testing Laboratory to asccrtain total solids and total iron content (as Fe). Results of the anaiysis are given in Table 1. Total iron decreased from 140 ppm at start up to 4.40 and $4.68 \mathrm{ppm}$ arter pumping 24 and 30 nours, respectively. Tnis Iron content is the same essencially as shown by samples trom Permanent Well No. 2 at the wacer treatment plant. Total solids also decreased during the test to a value somewnat lower than the values obcained from test samples of Well No. Z taken in Marcn, ipril and May.

(2) Biological lests - On 18. June 03 Sgt Dennis advised Mr. Ireton of cnis office that his blological test samples were all NEGATIVE. His test consisted of incubating the water samples for twency-four hours and examining the places for bacteria. THIS IS NOT AN ACCEPTED BIOLOGICAL TEST FOR WATER POTABILITY.

8. CONCLUSIONS:

a. Test Wells - Observations to date indicate that marked fluctuations occur in the test wells in the dissolved iron content, the total solids content, and the static water level.

(1) Fluctuations in Static Water Level - It has been observed that the static water level in the test wells rises with the rise in Yukon River water after the spring ice-breakup, and that the static water level falls when the river water returns to a lower elevation in early sumer. The rises and falls in the static water level of the test wells are closely tied in 


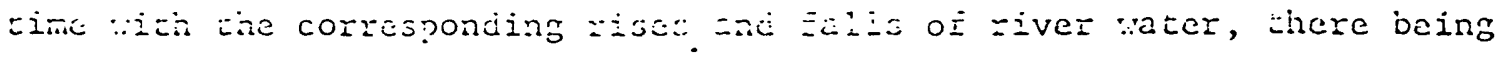
very Iittle lag between tham. Thiz :ould indicate that the sediments are higily perticable and permit easy roviment of river water toward the wells Fen the nyciralic gracient is in thio direction. At other times of year, scant evidence suggests thet the : thtic :icter levels of the test wells are at a higher elevation than that of the river vater, and that the hydraulic gradient is coward the south, causing rater to move from the wells to the river. The source of recharge to the risis during this part of the year rouid be the highlands to the rorth. Then the level of the Yukon River rises after the spring ice breat-lip, this water could entar the higher permeable strata lying above tha lower acuifers which contain water from the north. The static rise in witer level observed in the vells would then be due in part to this influx or infiitration of Yukon River water into the upper strata. The two groundwaters, pernaps differing markedly in quality, could remain lergeiy separated aiong a more or less horizontal interface. Some intermixing rould undoubtedly cake piacs however in the immediate vicinity of the interface. $\therefore$ vil with the screen set close to this interface vould tend to produce vater of variabie quality. Also, it may be inferzed that the rise in static water levels in the velis is attributabie in part to a rise in the groundvater level in the lower aquifers, resulting from increased movement of vatce from the north at the time of the spring tian:. The position of the screen relative to this apparcnt interface vould then become a critical factor in interpreting the fiuctuations in iron anc totai solids observed in the veils. 


\section{(2) Fuctuations in Eote: So:izis - Land to the north is almost}

level for about 15 miles before a rise to the highlands takes place. Much of this relatively level area consisus of iakes, swamps, and bogs. The hydraulic gradient rould be lov and gzoundwater movement toward the south v:ould be slow. It appears likeiy that sucin groundwater would contain a high amount of dissolved solids because of the long distance traveled and the slow movement which would allow time for more compleze solution of any soluble materials through wich the groundwater moved. At the present time there is a lack of data on the chemical quality of this vater to the north. It is assumed to be high in total solids for the purpose oi this discussion and as a possible explanation for observed fluctuations in total solids in the test wells. At Test WeIl W-348, the total solids content on 31 May 63 was considerably higher than during 5-13 April 63. This vould indicate a rise in groundwater level of the lower aquifers containing this water from the north. During the earlier pump test of 5 - i3 ipril 63, this well could have been drawing water from an upper iayer conjaining less total solids. At Test Well W-347, closer to the Yukon Rivar, the totai solids content on 3 June 63 was ipproximately the same $2 s$ on $1-\geq$ april 2963 . A condition of equilibrium vith respect to the upper and lower groundwaters could, thereFore, have been in effect between these tro dates insofar as the total solids content is concerned. Test Well :!-347 contained less than half the total soidds found in Test well $W-348$, but the content of total solids in Well 1:-347 is apparently considerably higher than that of the Yukon River. Chemical anaiyses of Iukon River water at Gaiena are lacking. The nearest point to 
Gaicna there systematic sampies of river vater have been taken is at Rampart wich is above the confluence of the Tanana and Yukon rivers.

\section{(3) Fluctuations in Iron Content - Fluctuations in the iron} content of the test wells are not considered in the foregoing discussion. The behavior of iron in the test vells is not well understood at this time. The ferrous state in which most of the iron exists in solution indicates a lack of oxygen. With sufficient oxygen, ferrous iron is converted to the ferric state and precipitates out of solution as ferric hydroxide. During the late spring and summer, with increased movement of groundwater and exposure of the ground surface to atmospheric oxygen, more oxygen may be present in the groundwater than during the winter months. The increased oxygen would precipitate some of the dissolved iron from solution and thereby lower the dissolved iron content. In addition, the uppermost layer of relatively iron-free groundwater, mentioned in the basic report as present at several test wells, would likely increase in thickness. If a significant increase in thickness occurs during the summer it could explain the lower content of iron which occurs on pumping at Well $\mathrm{W}-348$, and possibly at Well W-347. Pumping at these wells may draw a large portion of the water from the iron-free surface layer, and the iron content could fluctuate according to the thickness and oxygen content of this layer. These wells may also receive contribution of oxygen from the apparent infiltration of Yukon River water into the upper strata.

(4) Additional Data Requized - The tentative hypothesis outined in the preceding paragraphs requires additional data for confirmation 
of its validicy or its replacement by another hypothesis. The data needed are as follows:

(a) The direction of groundwater movement at various times of year with particular note of the time and extent of any reversals in this movement. These data would permit a firmer correlation of changes in well water with the source of recharge and the position of any interface between Yukon River vater and water from the north. The data could be accumulated by regular coincident determinations of the static water level in the test wells, the elevation of the Yukon River water, and chemical analyses of well water.

(b) Regular determinations of the oxygen content and $\mathrm{pH}$, as vell as dissolved iron and total solids, of test well water from specified depths. These data would enable the role of oxygen in lowering the dissolved iron content of well water to be evaluated more accurately, and a significant correlation of high oxygen with low iron content might be established. The data on dissolved iron and total solids could help to establish the presence of an interface and its behavior.

(c) A chemical analysis of groundwater from a point north of the station. Determination of the $\mathrm{pH}$ and the content of iron, total solids, and oxygen of this water is needed, and preferably of samples taken at several different time of year. These data could be obtained by means of a test well in this area, and such a well would also be useful in confirming a hydraulic gradient toward the south.

(d) Determine iron and total solids content at Yukon River a.eer at Gaìtna. 


\section{b. Permanent Vater Veil No. i}

The pumping test of tinis well, plus data on our records, indicates this well is satisfactory as a backup well for the Base potable water supply. To set up a well-engineered permanent-type backup supply, the following should be done:

(I) The existing overage pump in this well should be pulled and replaced. It should be replaced with a submersible pump so that the Fireman's office space will be uncluttered. Use of a pump having a capacity of about $100 \mathrm{gpm}$ at the T.D. head for discharge through the treatment plant iron precipitator will be appropriate.

(2) Piping to the head of the well should be kept underfloor and the casing protected with a carefuliy insilled sanitary seal.

(3) At the time the pump is replaced, the well should be cleaned and the screen checked so that a satisfactory well-service life of another 10 - 15 years will be possible.

Emergency Use - If the present unsanitary and unsealed conditions at the well head were rectified, this well coule be used as is for an emergency raw water source for delivery to the water treatment plant. Water should not be used from this well without chlorination, however. The probable capacity of this well, as is, when connected to the water treatment plant would be around $35-40 \mathrm{gpm}$. Therefore, Base use might have to be slightly curtained in order to live with this emergency quantity. 
(4) The snemical composition of the water from this well is essentially the same as that from Well No. 2 at the Water Treatment Plant; therefore, readjustment of the treatment process probably will not be necessary when switching from Well No. 2 to Well No. 1.

$$
* * * * * * * *
$$


TABLE 1

CHEMICAL ANALYSES OF WITER FROM GALENA AIRPORT

Results in PPM, Analyses by F\&M Branch, Testing Section

Sample No.

Date \& Time Sampled

Total Solids

Total Iron

TEST WELL NO. W-347 - Adjacent to Alert Hangar - raw water.

$\begin{array}{rrrr}38 & 3 \text { June } 63,1010 & 606 & 28.5 \\ 39 & 3 \text { June 63, 1310 } & 498 & 9.0 \\ 40 & 3 \text { June } 63,1610 & 500 & .8 .0\end{array}$

TEST WELL NO. W-348 - Adjacent to Water Treatment Plant - raw water
41
31 May 63, 0920
1664
1340
64
42
31 May 63, 1240
1320
0.25
31 May 63,1520
0.35

PERMANENT WATER WELL NO. 1 - At Fire Station - raw water.

$\begin{array}{lllr}44 & 7 \text { June } 63,1000 & 776 & 140 . \\ 45 & 8 \text { June } 63,1000 & 298 & 4.40 . . \\ 454 ! 0 & 8 \text { June } 63,1600 & 212 & 4.68\end{array}$

$12 a$ 


\section{TABLE 2}

SOIL CLASSIFICATION

Dentr (ft) Group Symbol

Classification Frost Susceptibility

TEST VELL NO. W-345 (Ammo Storage Building)

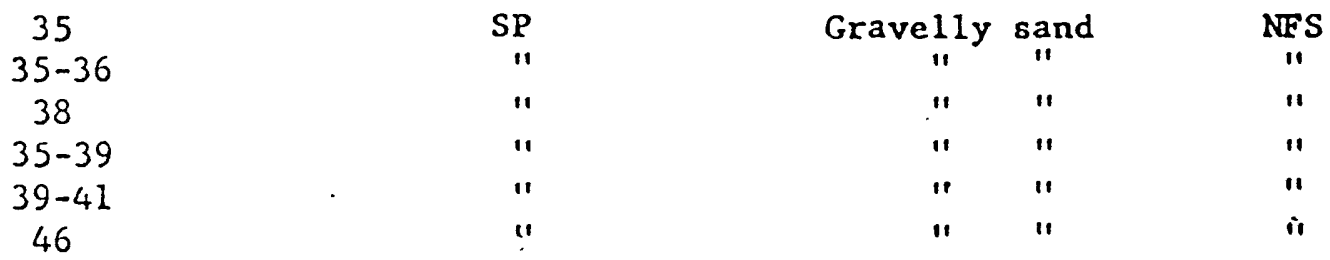

TEST I.ELL NO. W-346 (Guard House)

$\begin{array}{cccc}33-35 & \text { GP } & \text { Sandy gravel } & \text { NFS } \\ 33-54 & 11 & " 1\end{array}$

TEST VELL NO. W-347 (Alert Hangar)

$\begin{array}{lrlc}31-33 & \text { SP } & \text { Gravelly sand } & \text { NFS } \\ 31.5 & " & \text { Sand } & " 1 \\ 32-34 & " & \text { Gravelly sand } & " 1 \\ 34-35 & " & \text { " }\end{array}$

TEST WELL NO. W-348 (Water Treatment Building)

36.5

38

40
SP

"1
Gravelly sand

II
NFS

II 
TABLE 3

PURP TEST OF TEST TELI NO. $\mathrm{N}-347$

GALENA SIERT ILIYGAR

G:IIENA AIS

3 June 1963

$\begin{array}{llc}\text { Iinc } & \text { Water } & \text { Pump } \\ \text { Ievel } & \text { Pressure }\end{array}$

1010 hes

\begin{tabular}{|c|c|c|}
\hline 1030 & " & $4^{\prime} 1^{\prime \prime}$ \\
\hline 1045 & $"$ & $4^{\prime} 1^{\prime \prime}$ \\
\hline 1100 & $" 1$ & $4^{\prime} 1^{\prime \prime}$ \\
\hline 1115 & " & $4^{\prime} 1^{\prime \prime}$ \\
\hline 1130 & $"$ & $4^{\prime} 1-1 / 2^{\prime \prime}$ \\
\hline 1200 & $"$ & $4^{\prime} 1-1 / 2^{\prime \prime}$ \\
\hline 230 & " & $4^{\prime} 2^{\prime \prime}$ \\
\hline 1300 & 18 & $4^{\prime} 2^{\prime \prime}$ \\
\hline 1310 & $"$ & -- \\
\hline 545 & $"$ & $4^{\prime} 4::$ \\
\hline 500 & $" 1$ & $4^{\prime} \quad 4^{\prime \prime}$ \\
\hline 510 & "1 & -- \\
\hline
\end{tabular}

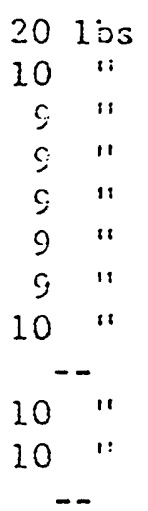

Started test - water sample if 1 . taken.

12 Some fine sand.

12.44

12.44

12.44

$12.4: 4$

22.44

12.44

12.44

$--$

12.44

12.44

11 " 11

1121120

11211

13 18 11

311011

112011

111111

Water sample 42 .

Sone fine sand.

" " "

liater Sample 满3.

All measurcments taken from top of casing $18^{\prime \prime}$ sickup.

Static nater level $31^{\prime \prime}$ from top of casing.

Syers H.C.M. Ejecto Pump, Serial No. 460-62, Century motor type C.S. $3 / 4$ HP, PH I, cycle 60, PMP 3450, Volt 115/230.

Pump left installed in open by Major Linton's request.

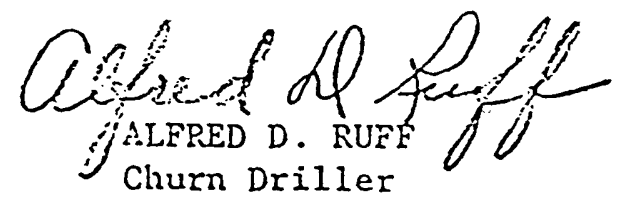


TABLE 5

PUMP TEST OF PERMANENT WELL NO. 1

FIRE S'LATION WELL

GALENA AFS

7-10 JUNE 1963

Date \& Time 1001

1100

1200

1300

1400

1500

1800

2000

2100

2

8 June $63-0600$

0700

0800

0900

1000

1100

1200

1300

1400

1500

1600
7 June $63-1000$
Water Level

5' 3" Static

$7^{\prime} 3^{\prime \prime}$ Pumping

$7^{\prime} 3^{\prime \prime}$

$7^{\prime} 6^{\prime \prime}$

$7^{\prime} 9^{\prime \prime}$

7' 6"

$7^{\prime} 6^{\prime \prime}$

$7^{\prime} 7^{\prime \prime}$

$7^{\prime} 6^{\prime \prime}$

$7^{\prime} 9^{\prime \prime}$

$7^{\prime} 10^{\prime \prime}$

$7^{\prime} 11^{\prime \prime}$

$8^{\prime} 0^{\prime \prime}$

$8^{\prime} 3^{\prime \prime}$

$8^{\prime} 5^{\prime \prime}$

$8^{\prime} 2^{\prime \prime}$

$8^{\prime} 3^{\prime \prime}$

$8^{\prime} 2^{\prime \prime}$

$8^{\prime} 2^{\prime \prime}$

$8^{\prime} 3^{\prime \prime}$

$8^{\prime} 4^{\prime \prime}$
GPM

Water green

46 Water sample No. 1

46

46

46

46

46

46

46

46

46

46

46

46

46

46

46

46

46

46

46

Remarks

Clear.

$$
\text { ". }
$$

clear.

"1

"1
Clear - water sample taken by medical technician.

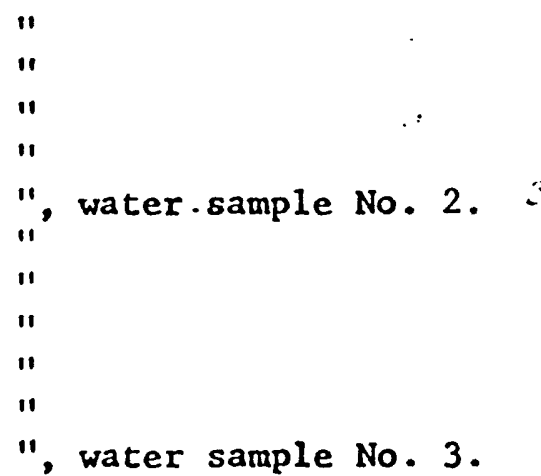

10 June $63-0800$

$-$
"Essentially

the same quantity".

Run from 1600 hrs

8 June to $0800 \mathrm{hrs}$

10 June continuously

by Base CE forces.

NOTE:

Increase in drawdown is attributed to concurrent fall in Yukon River level. 


\section{TABBLE 6}

CHEMICAL ANALYSES OF YUKON RIVER WATER AT RAMPART, 1960-61, IN PARTS PER MILLION*

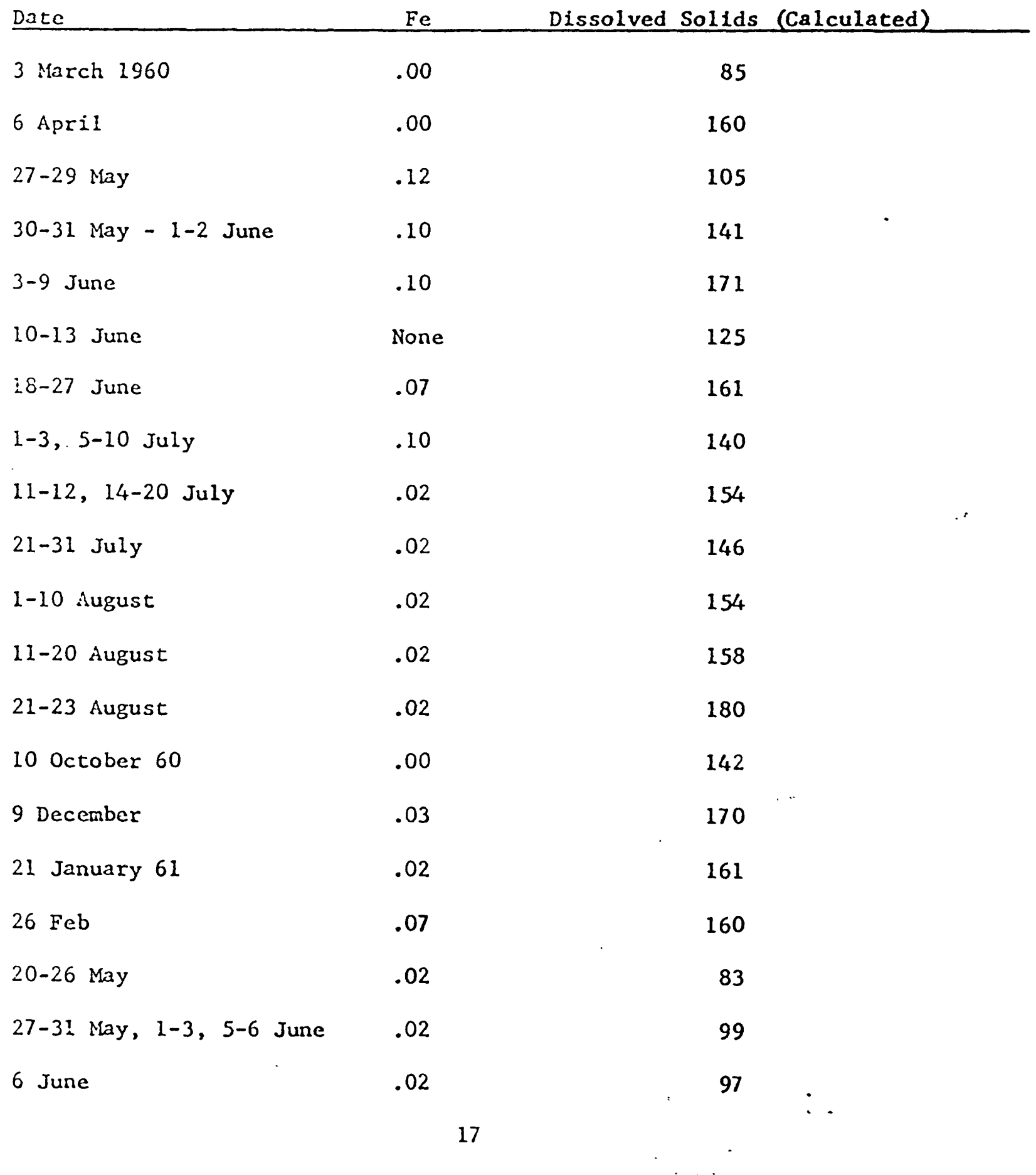




$\begin{array}{lcc}\text { Datc } & \text { Fe } & \text { Dissolved Solids (Calculated) } \\ \text { 10-i8 June } & .02 & 105 \\ 19,24-30 \text { June } & .02 & 153 \\ 1-8,10 \text { July } & .02 & 161 \\ \text { i1, 15-22 July } & .03 & 137 \\ 23-31 \text { July } & .05 & 134 \\ 1,3-7, \text { 10 August } & .03 & 151 \\ 11-20 \text { August } & .02 & 139 \\ 21-28,30-31 \text { August } & .02 & 176 \\ & \end{array}$




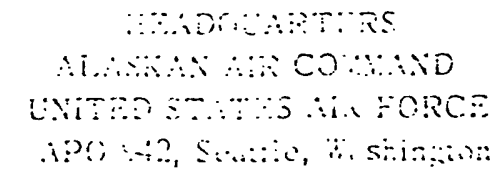

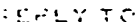

$\therefore \div-\because こ F$

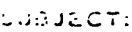

ALDEC-3D

yater Velis (Observation) Gaiena AFS, Alaska, project No. GiL $72-3$ ( $\mathrm{E} \in \mathrm{V}$ 2)

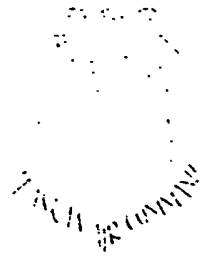

13 JUN 1963
roject No

$\because$ :Lasisa Distict Engineer (

1. Reference is made to FY 1963 Design Instiuction Number MC-1/63-iZI7-A/Cr/4, Galena Airport, Alaska, dated 10 August 1962, paragiaph " $b$ ", which authorizes the construction of observation vells for the purpose of securing information on the quantity and quality of water at various depths.

2. Consideration is being given to reactivate the presently uaused well No. I in Fire Station Building 1549 to elininate our presentiy complate cependence on a single

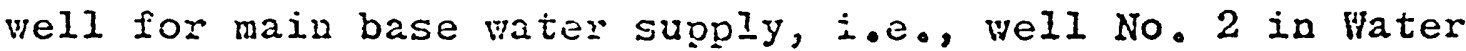

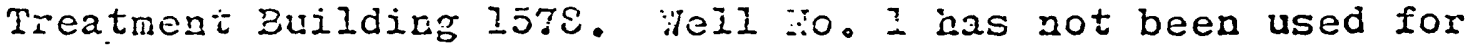
some considerable time for reason of a reputed but unproven contanination.

3. Request a seventy-::0 hour pumping test be performed on this well to prove tie jype and degree of contamination, and determine whether it can be reactivated. Air Force personnel will wake bacterial studies while the pumping test is underway. Water being punped way be wasted into a nearby drainage ditch so as not to overload the sewage plant during the test.

4. Since you bave a well crew at Galena, it would be most advantageous and economical to have the test made at this rime. 545-2526 funds remaining from the observation well project may be utilized for this work in the amount oI $\$ 600$. This authorization will not be exceeded without priol approval from this headquarters.

5. Request you proceed with this work as soon as arrangements can ie completed.

FOR TET COMIKANDER
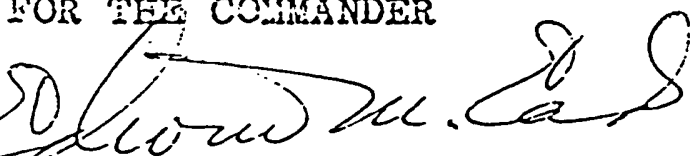

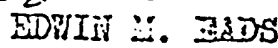

Colonel, USAR

TCS/Citil Enrinannin: 


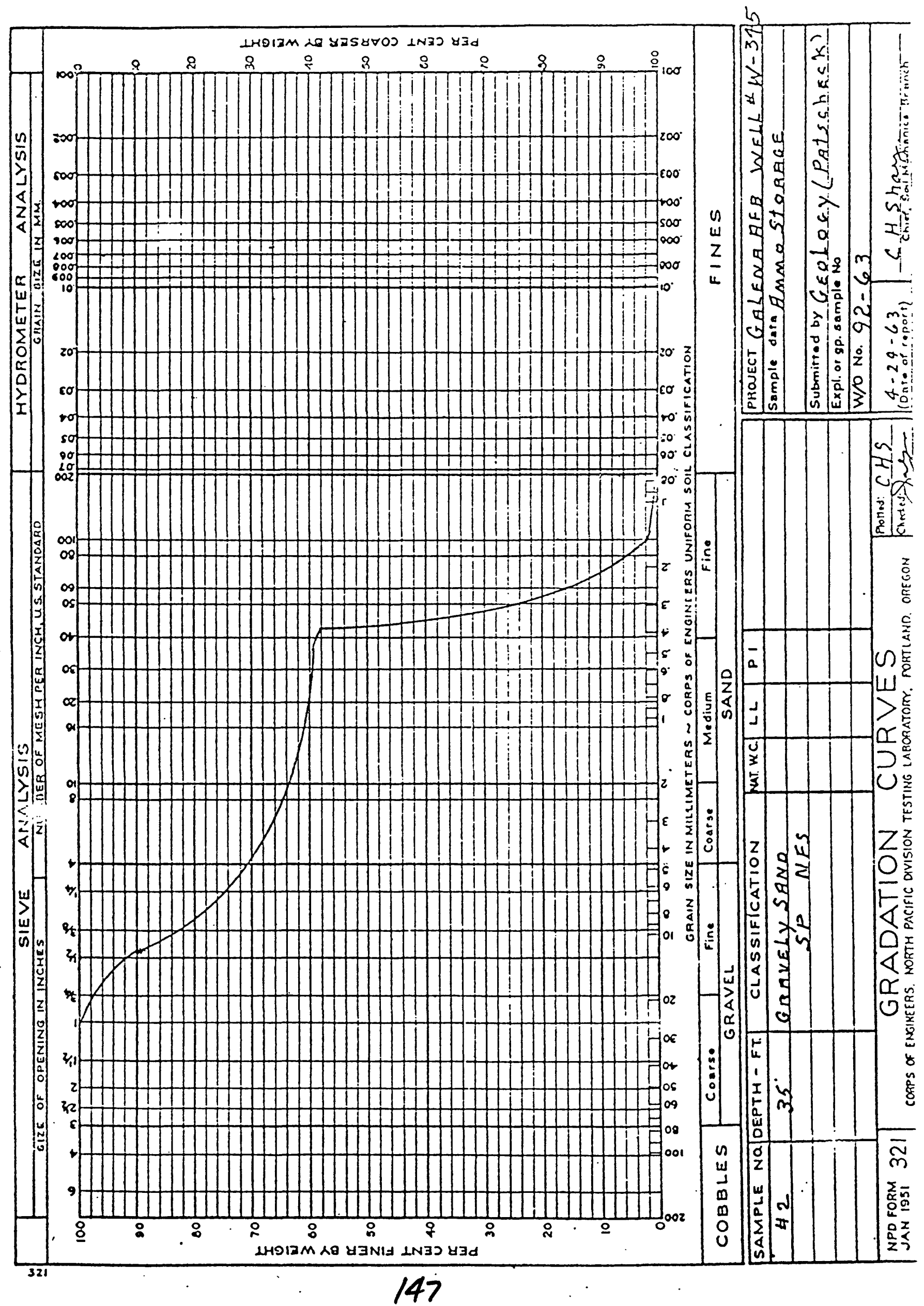




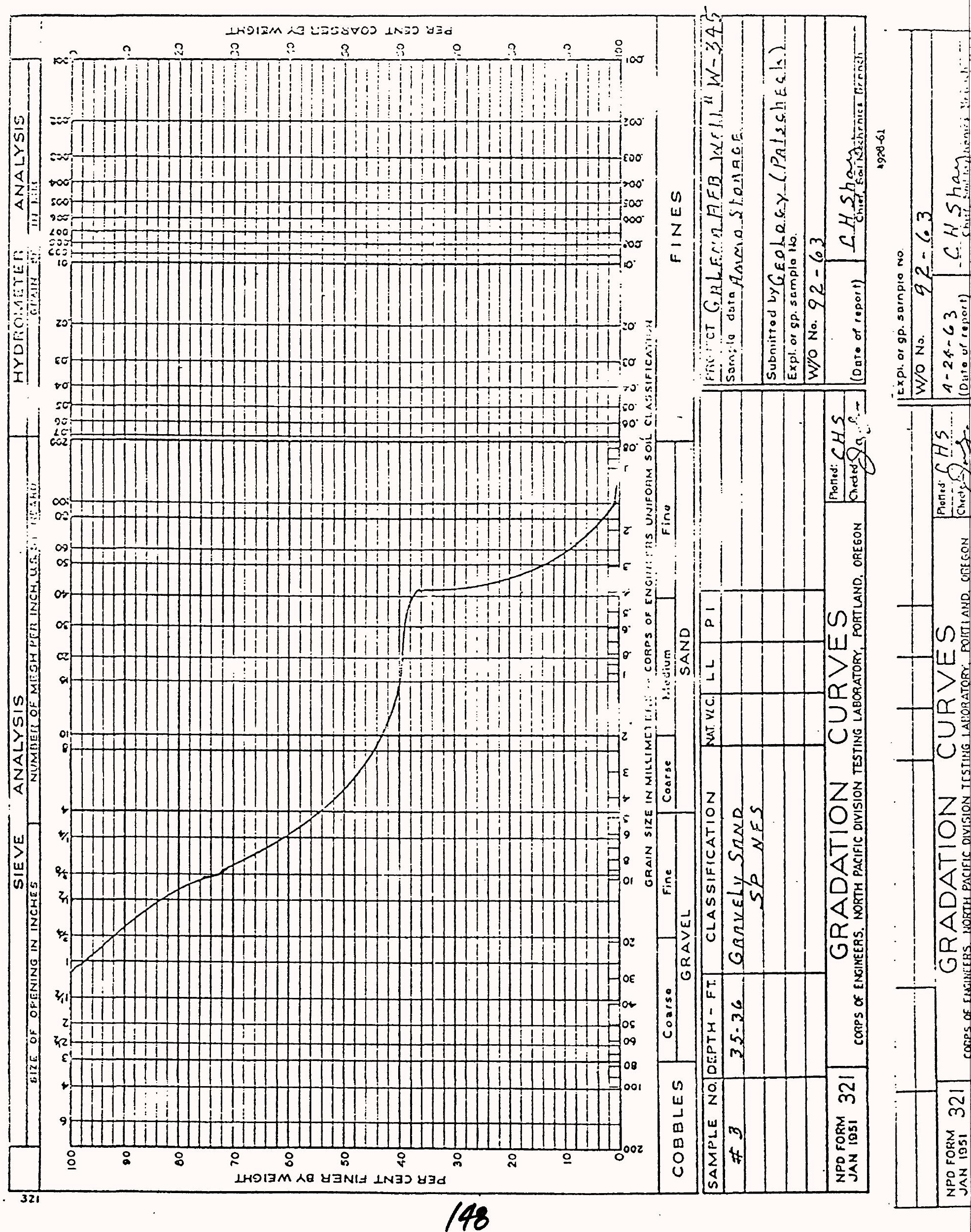




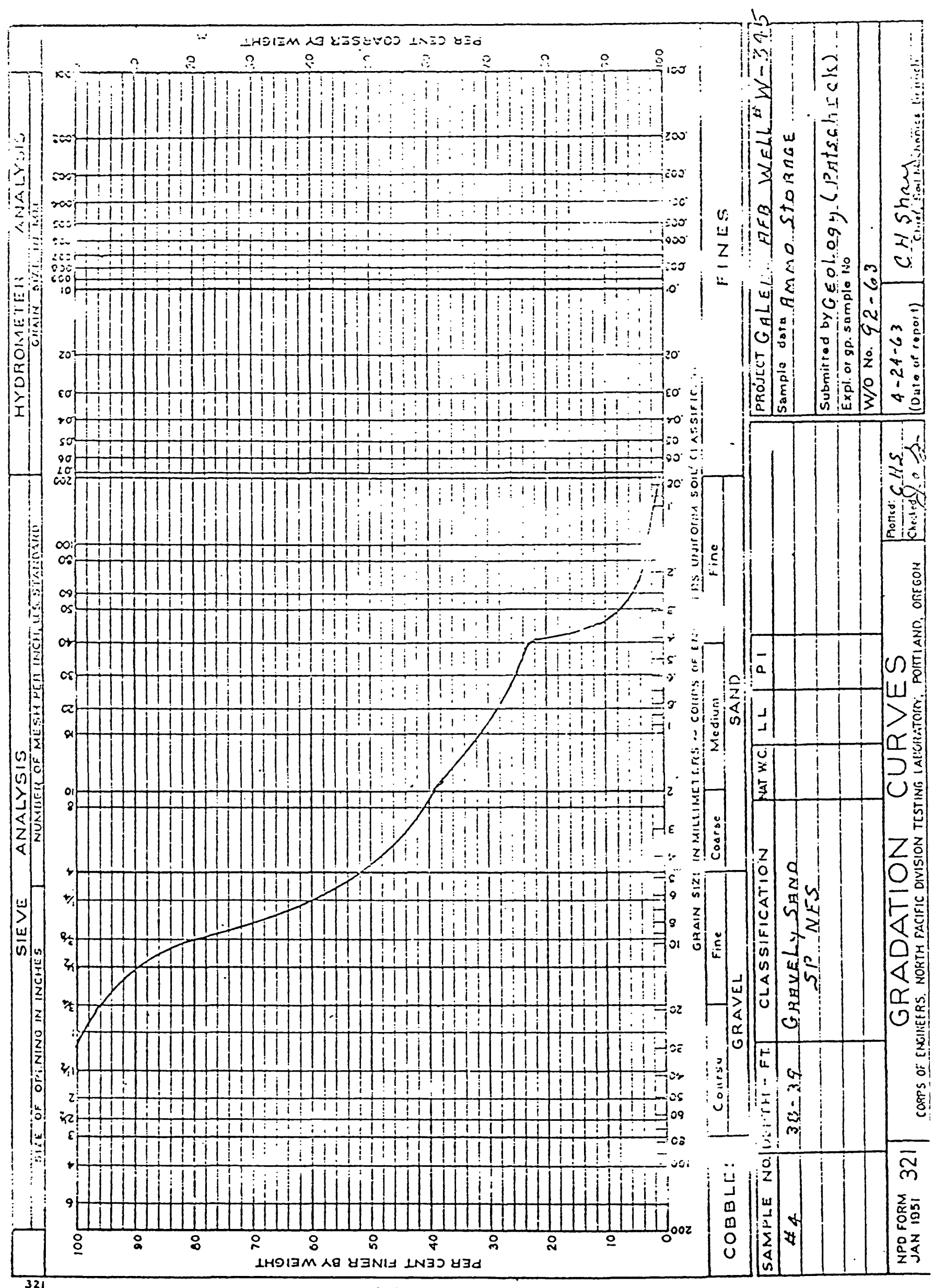



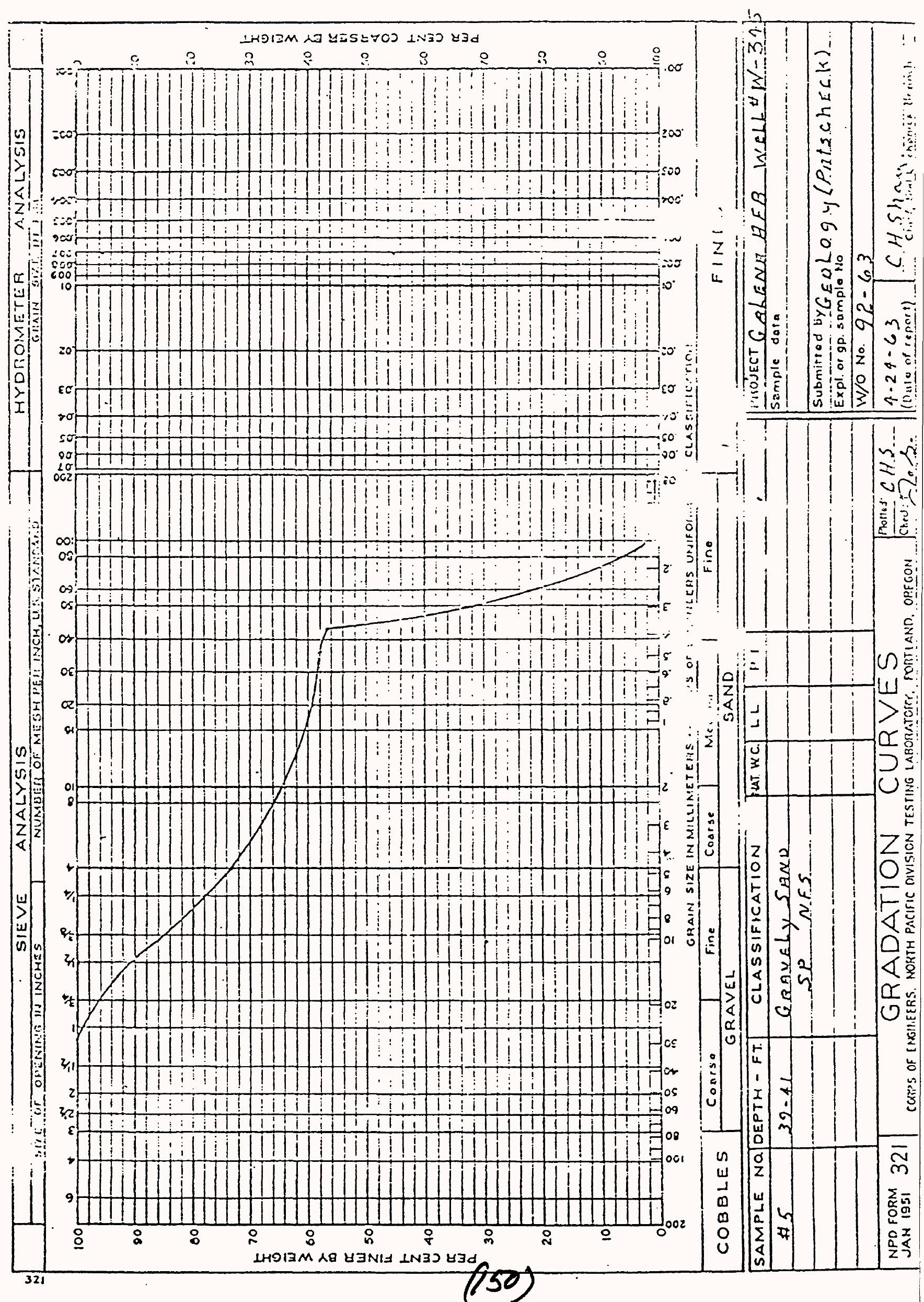


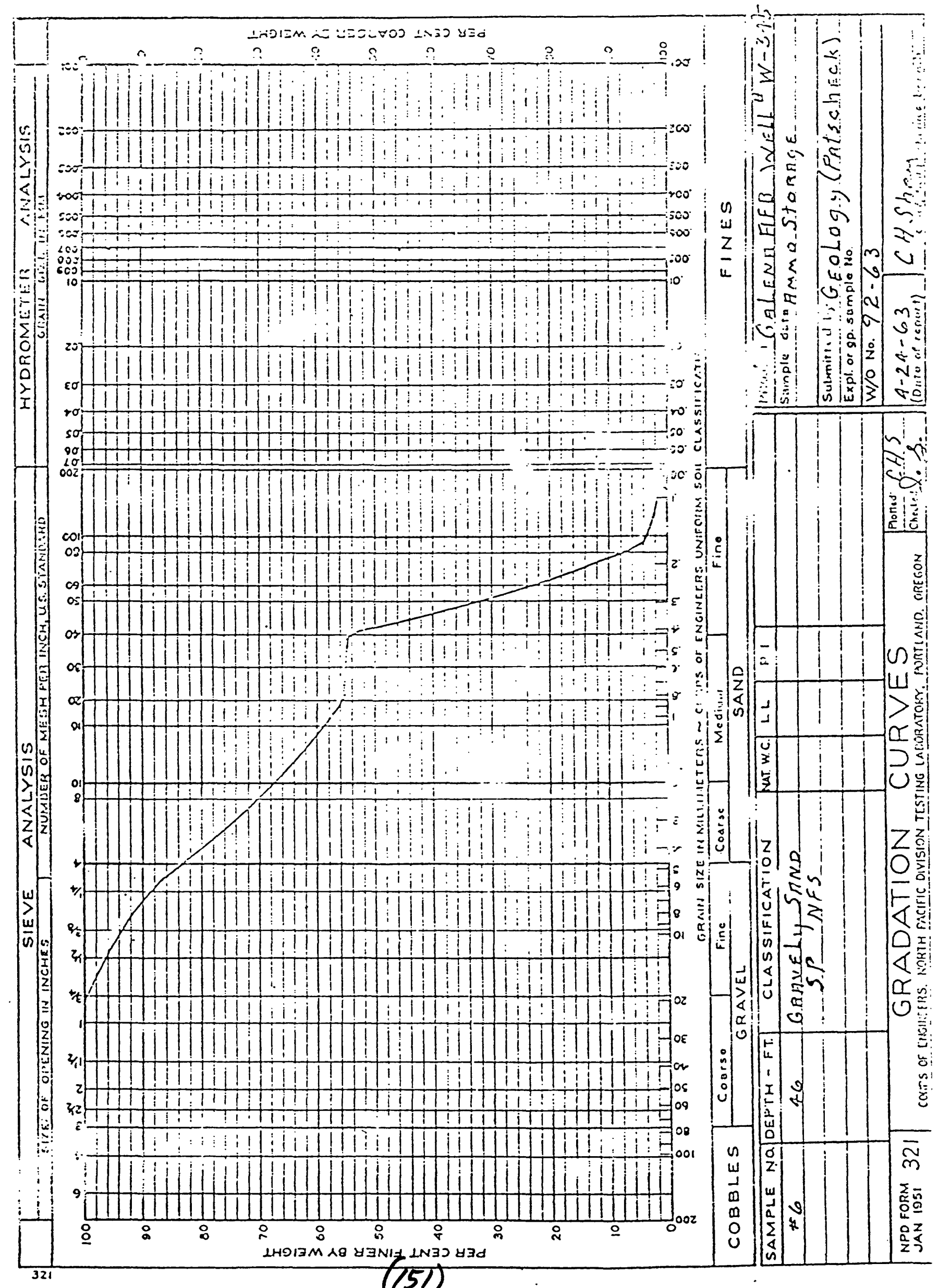




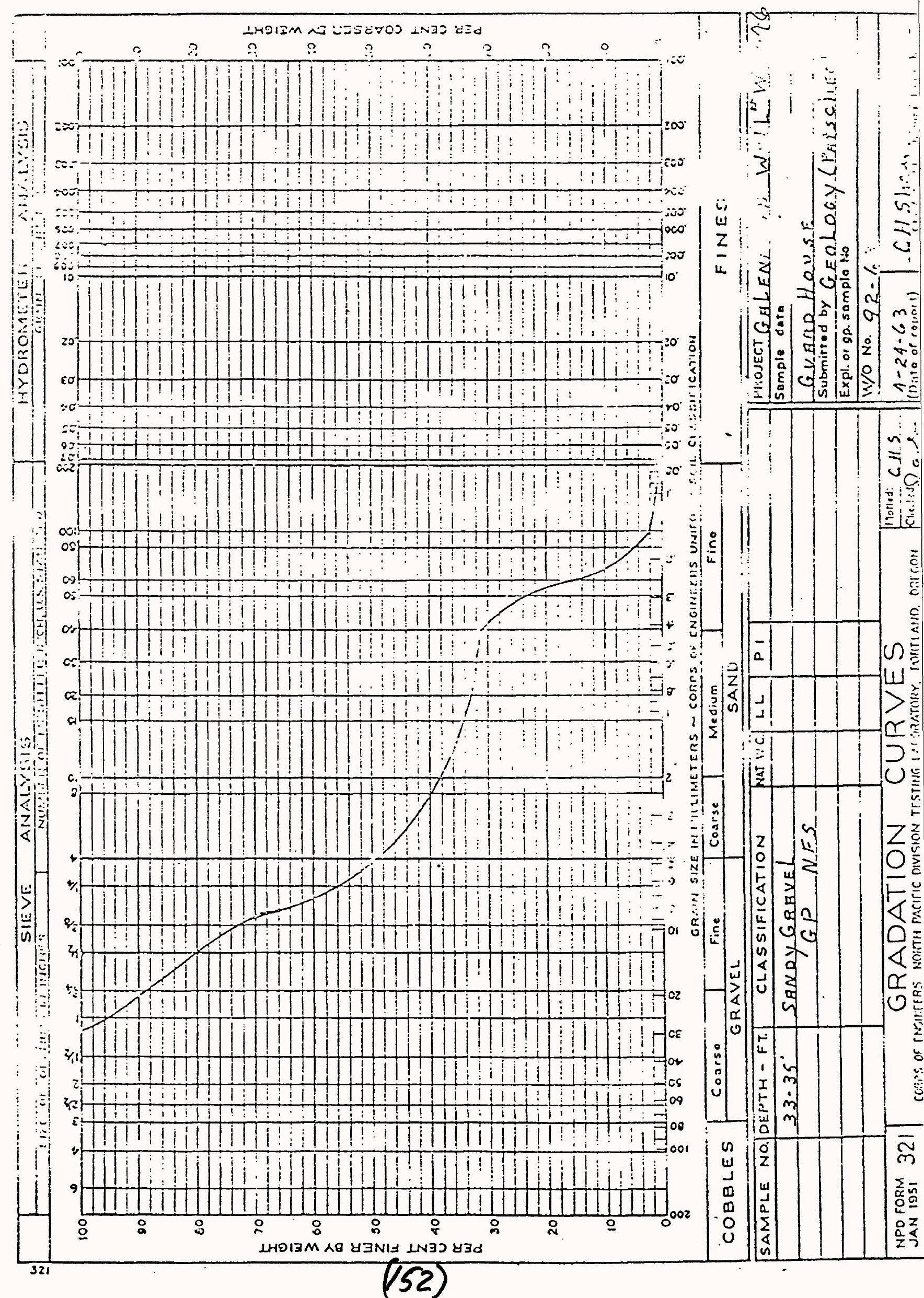









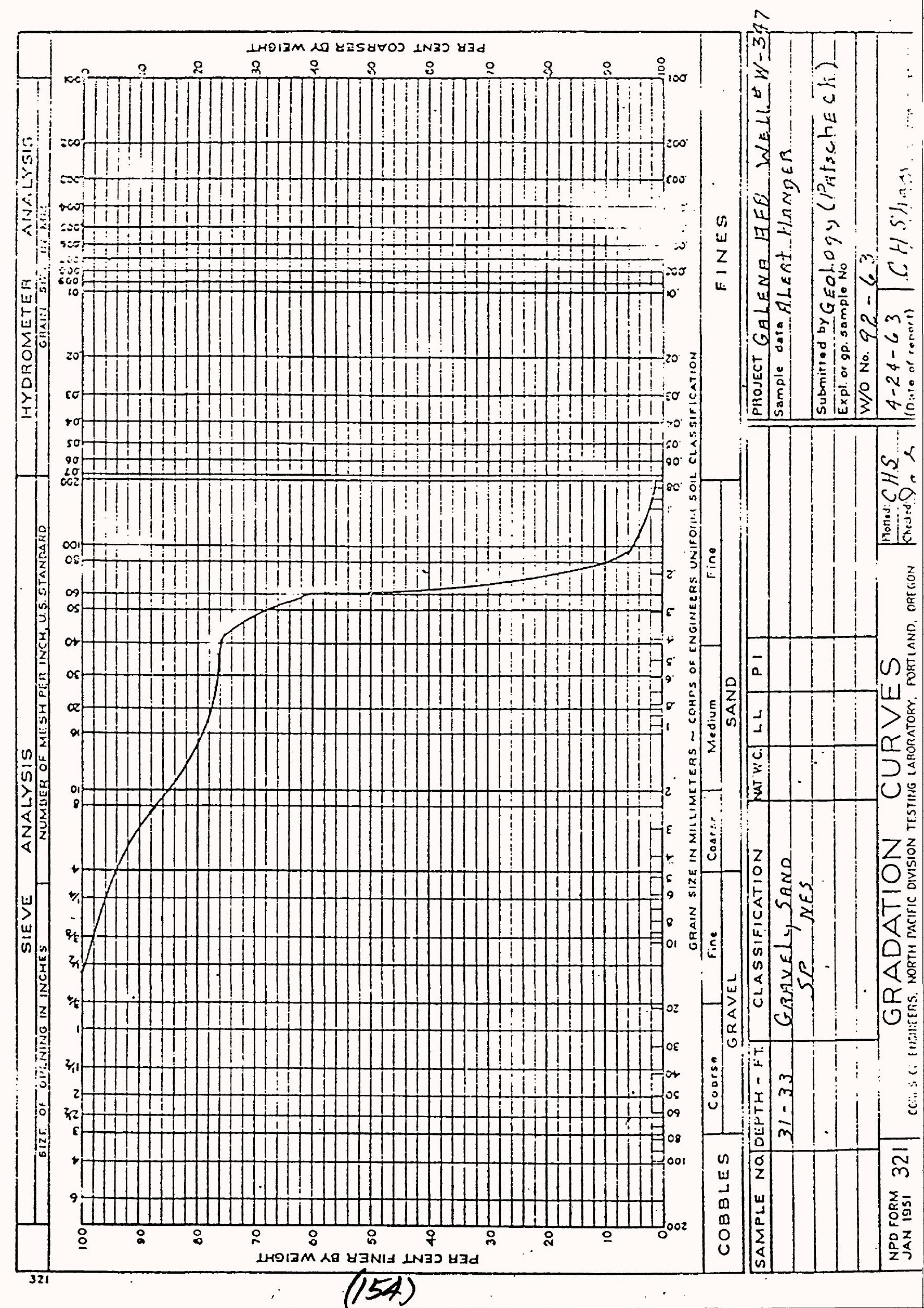




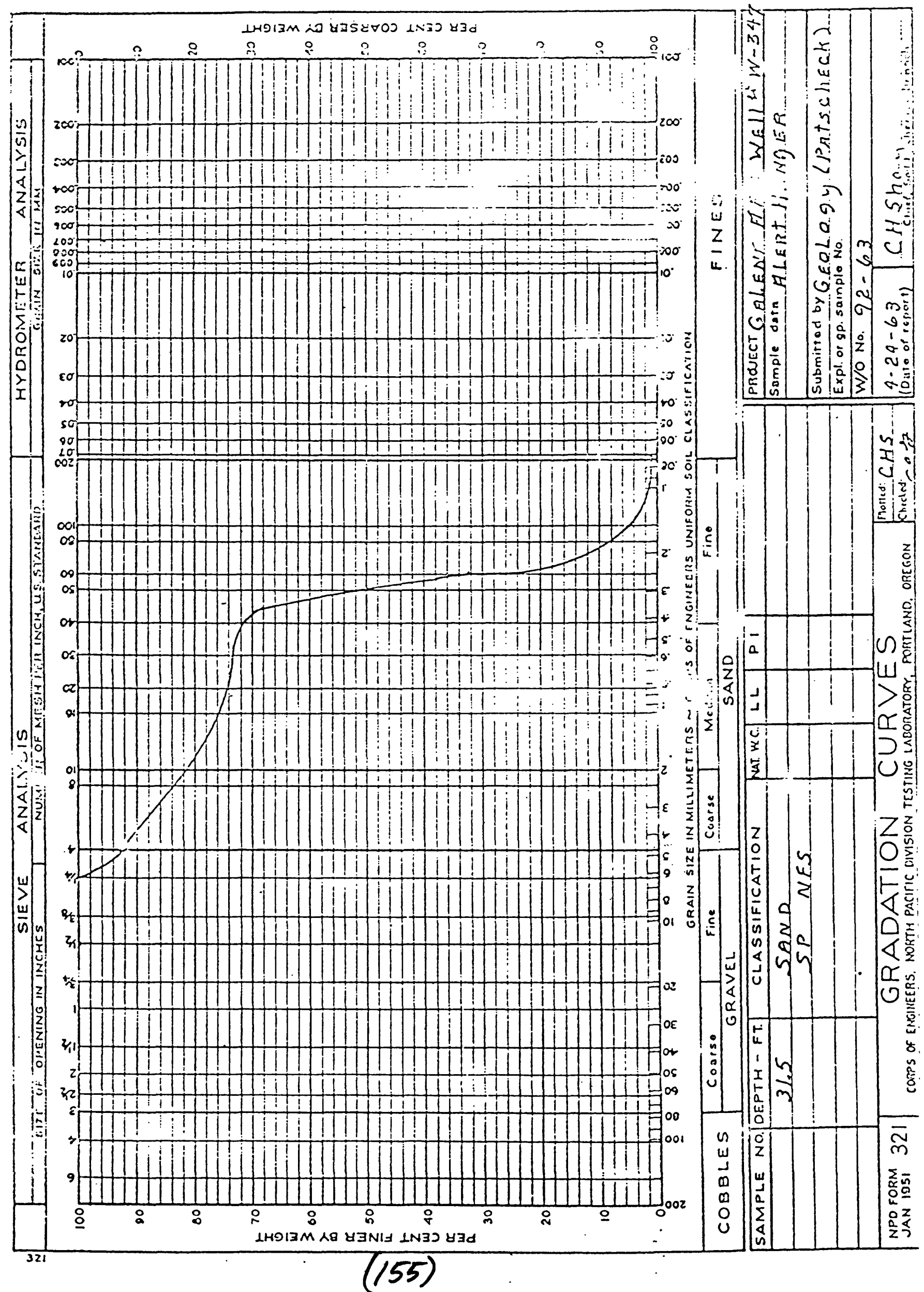




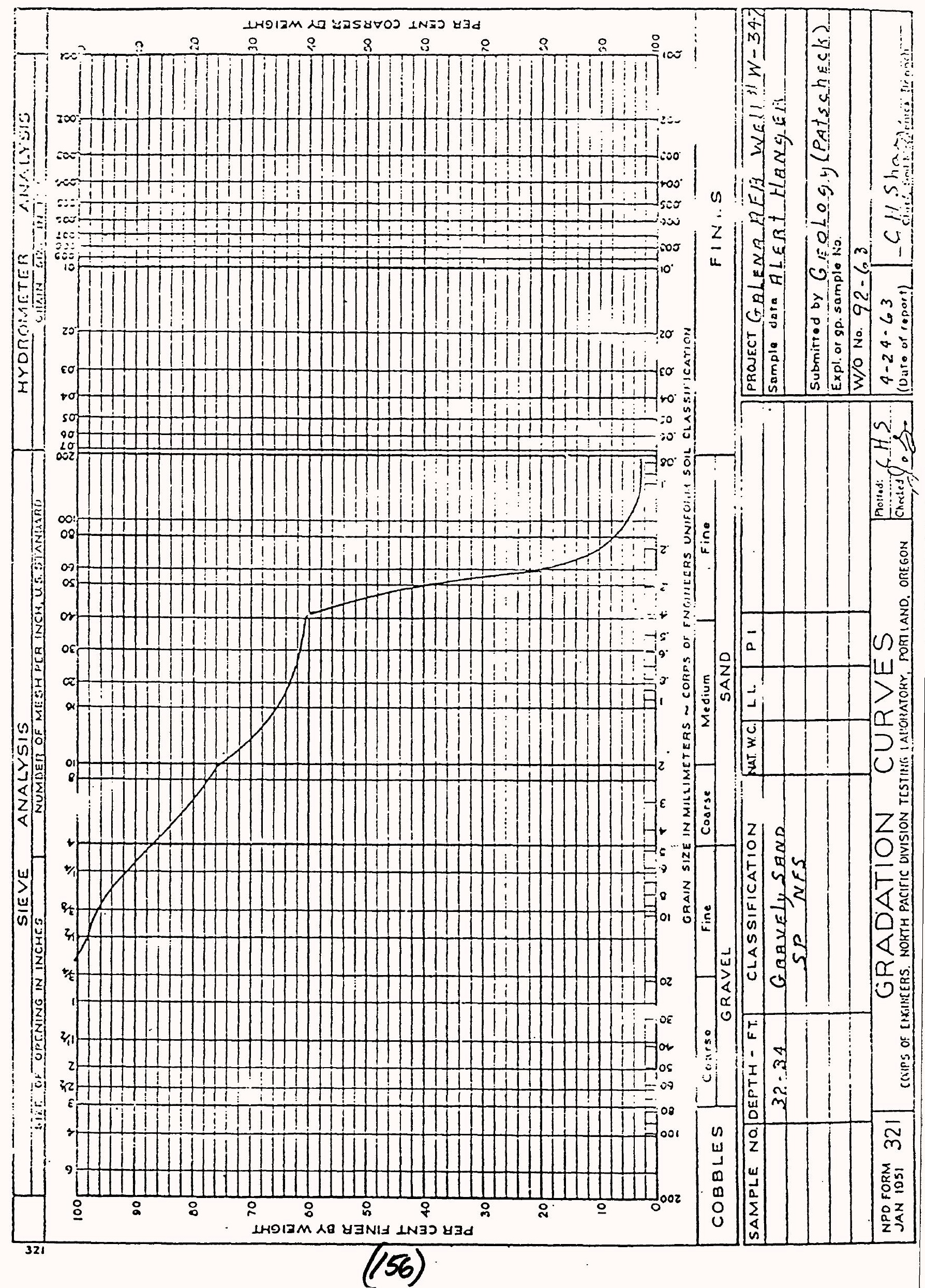









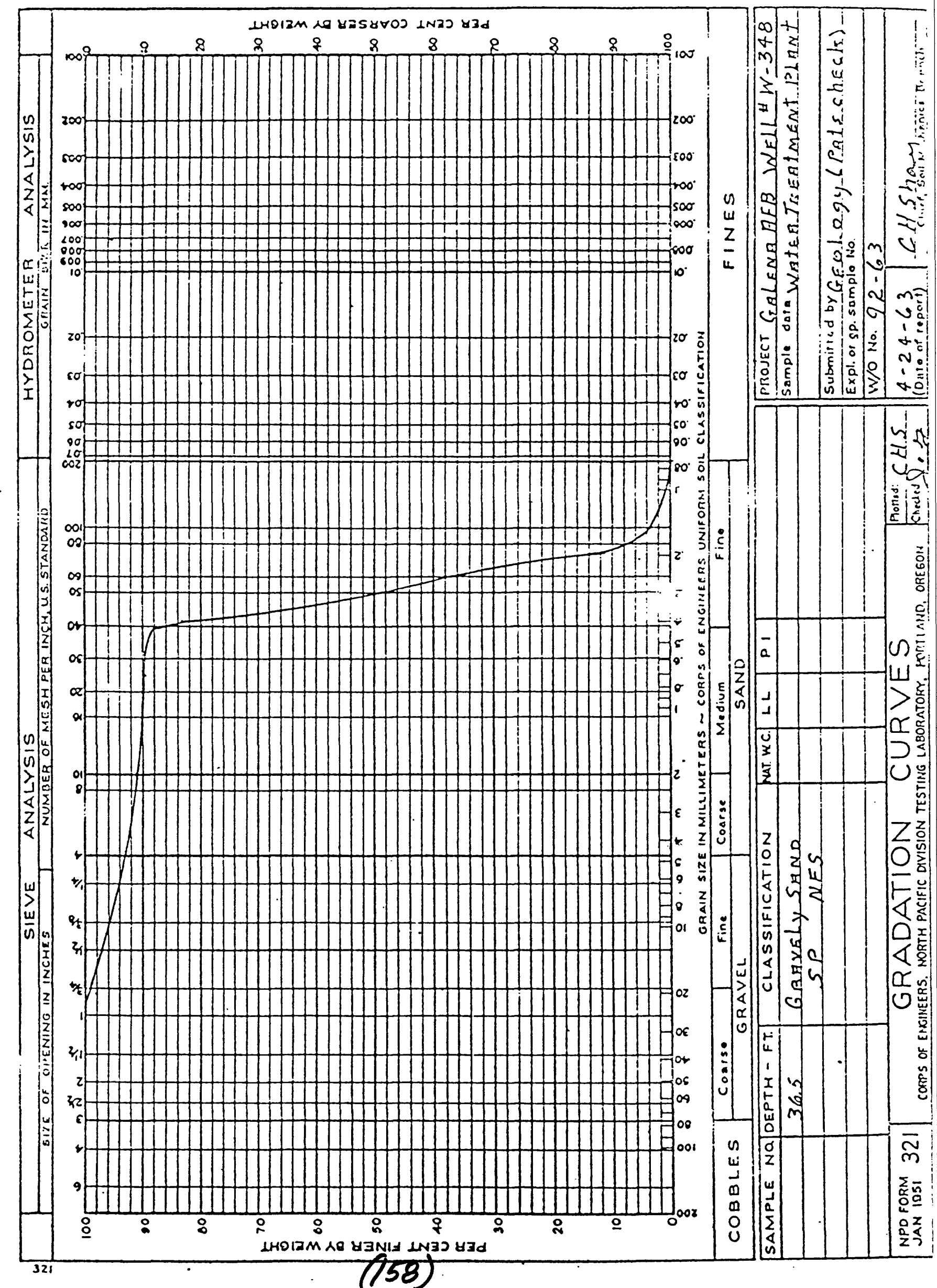




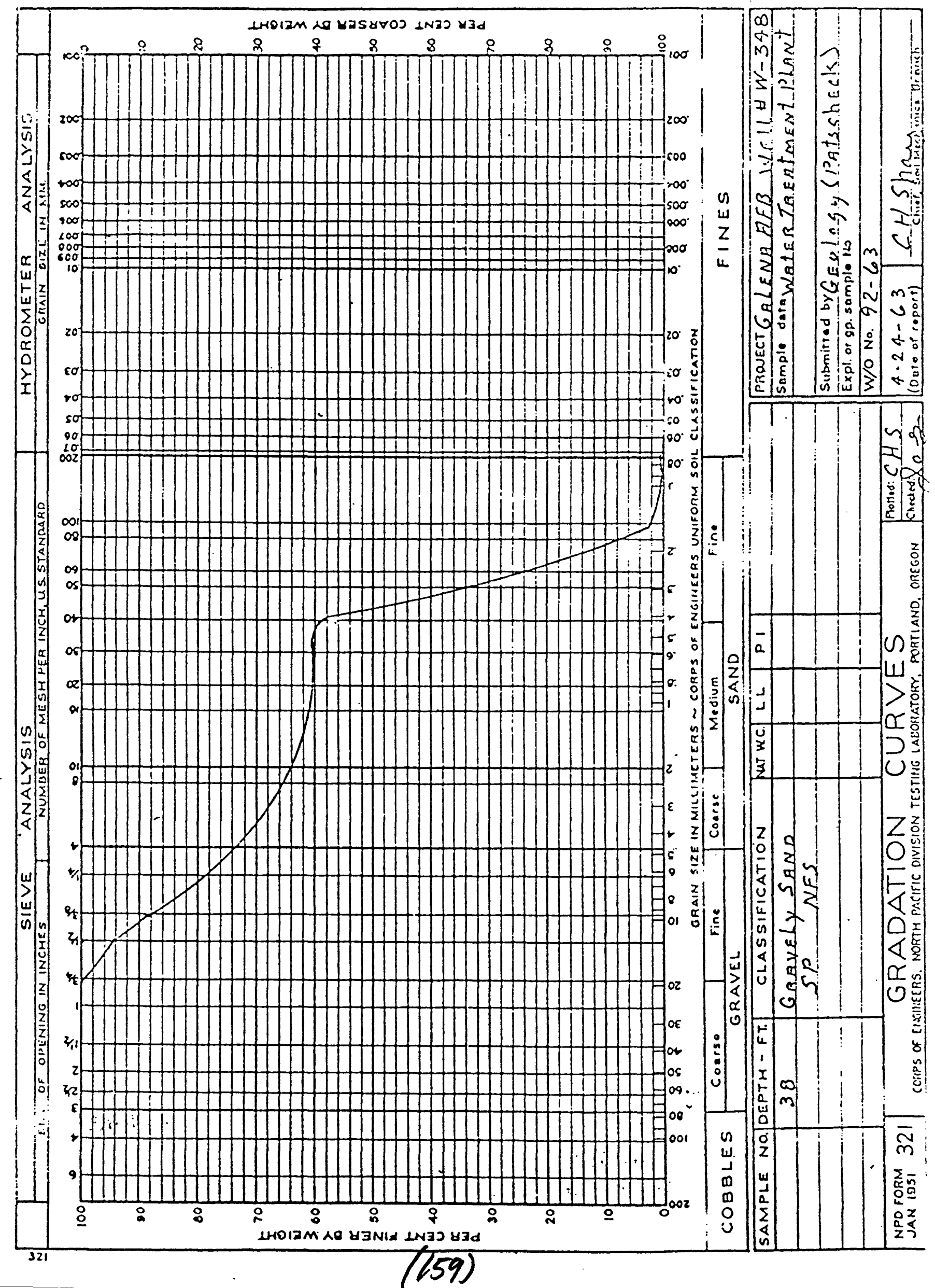




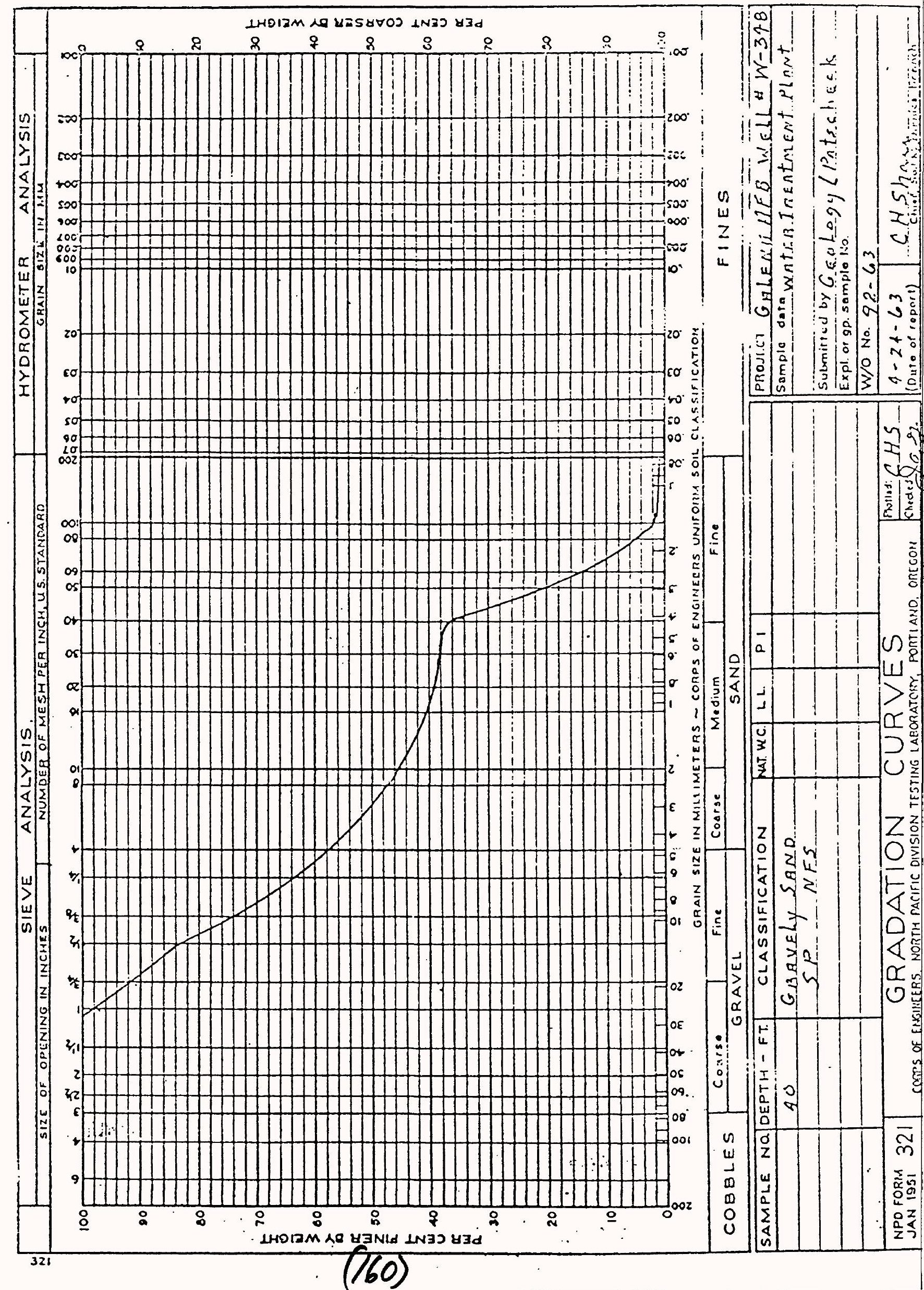




\section{APPENDIX 7}

U.S. Geological Survey water quality data for the Yukon River at Ruby and the Yukon River at Galena 

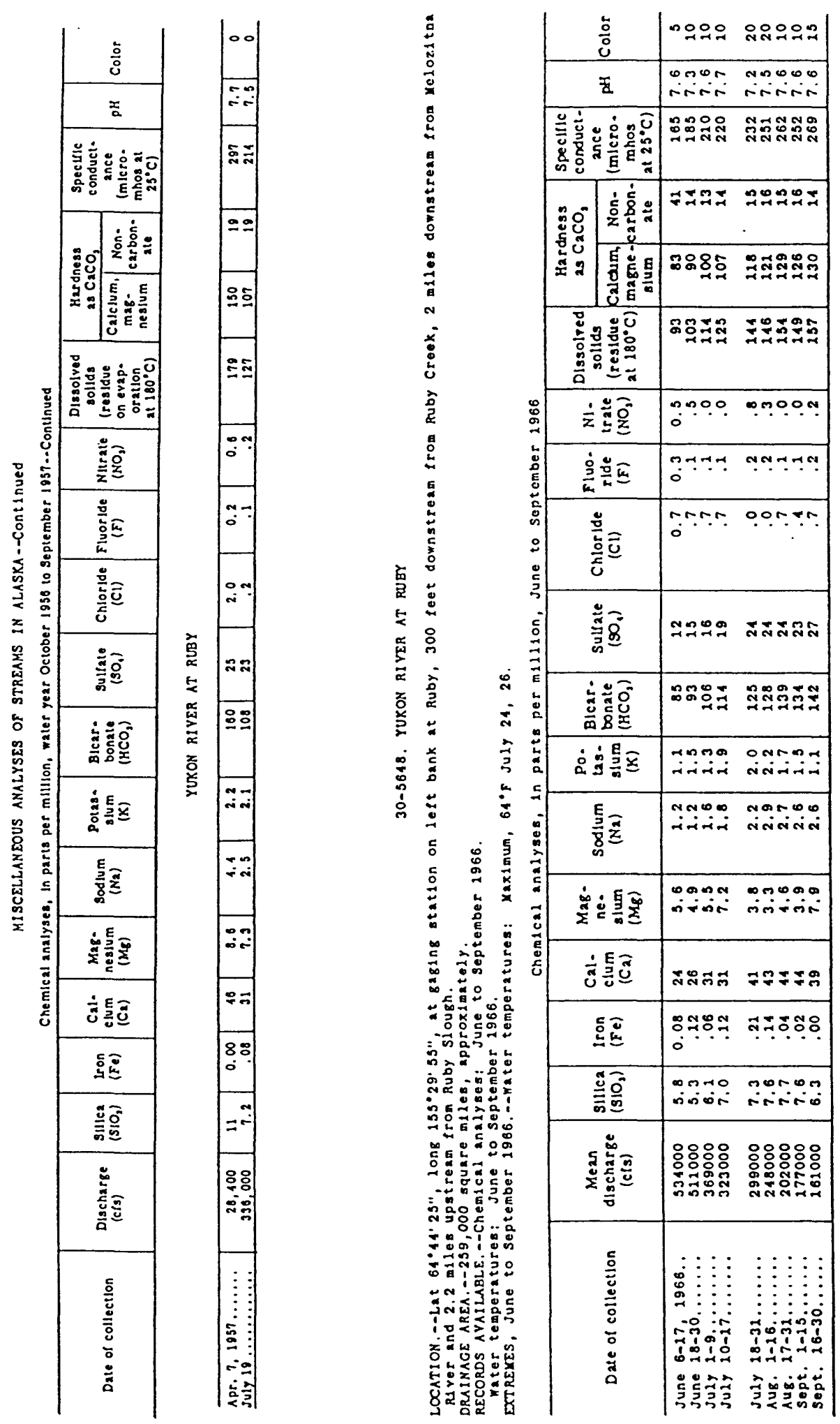


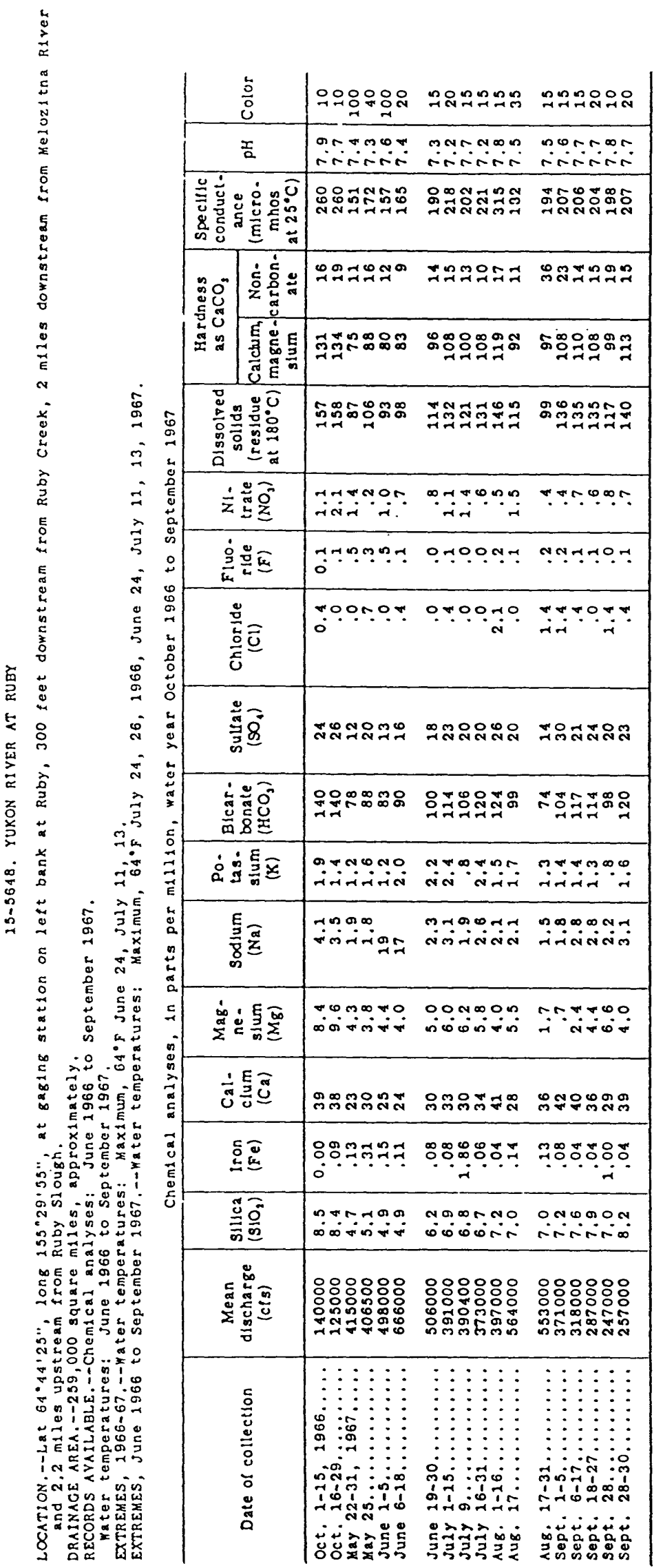


15-5648. YUXON RIVER AT RUBY

(International Hydrologic Decade Station)

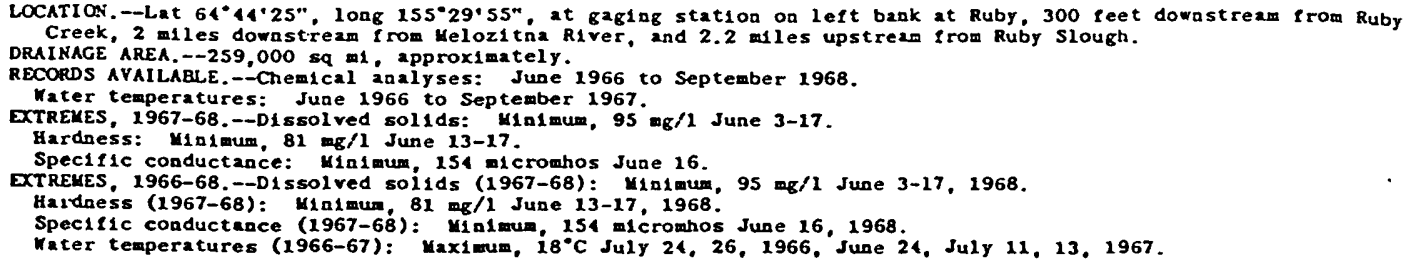

Chemical analtses in mILligrans per liter, water vear october 1967 to september 1968

\begin{tabular}{|c|c|c|c|c|c|c|c|c|c|}
\hline DATE & $\begin{array}{l}\text { DIS- } \\
\text { CHARGE } \\
\text { ICFSI }\end{array}$ & $\begin{array}{l}\text { SICICA } \\
\text { ISIOZII }\end{array}$ & $\begin{array}{l}\text { TOTAL } \\
\text { IRON } \\
\text { IFEI }\end{array}$ & $\begin{array}{l}\text { CAL- } \\
\text { CluY } \\
\text { ICAI }\end{array}$ & $\begin{array}{l}\text { MAG- } \\
\text { NE- } \\
\text { SIUM } \\
\text { ( MG) }\end{array}$ & $\begin{array}{l}\text { Soolum } \\
\text { (NA) }\end{array}$ & $\begin{array}{l}\text { Po- } \\
\text { ras- } \\
\text { SIUA } \\
\text { (K) }\end{array}$ & $\begin{array}{l}\text { DICAR- } \\
\text { GOMATE } \\
\text { IHCOSI }\end{array}$ & $\begin{array}{l}\text { SULFATE } \\
\text { (SO4) }\end{array}$ \\
\hline \multicolumn{10}{|l|}{ oCr. } \\
\hline O1-OS & 33 & 9.2 & -- & 38 & 6.1 & 2.9 & 1.1 & 130 & 23 \\
\hline $06-14$ & 108 & 9.0 & .01 & 36 & 7.1 & 2.5 & 1.4 & 125 & 10 \\
\hline $15-11$ & 150 & 9.4 & . is & 39 & 8.0 & 5.2 & 1.9 & 130 & 20 \\
\hline \multicolumn{10}{|l|}{ JUNE } \\
\hline $02 \ldots$ & 48 & 5.2 & -55 & 27 & 47 & 1.5 & 1.3 & 03 & 14 \\
\hline $03-17$ & 614 & 5.5 & $\ldots$ & 25 & 4.5 & 1.5 & 1.1 & 87 & 13 \\
\hline $18-27$ & 440 & B. 0 & -- & 26 & 5.3 & 2.0 & 1.1 & 92 & 17 \\
\hline $28-30$ & 431 & 6.2 & -- & 27 & 5.6 & 2.2 & 1.1 & 95 & 19 \\
\hline \multicolumn{10}{|l|}{ sulr } \\
\hline $01-02$ & 420 & 8.2 & -- & 27 & 5.6 & 2.2 & 1.1 & OS & 19 \\
\hline $0 B-17$ & 339 & B.6 & - & 31 & 6.1 & 2.6 & 1.4 & 110 & 22 \\
\hline$(8-2)$ & 308 & 6.9 & -- & 35 & 6.1 & 2.4 & 1.7 & I & 22 \\
\hline $28-31$ & 215 & 1.0 & -- & 34 & 6.0 & 2.4 & 1.7 & 119 & 24 \\
\hline \multicolumn{10}{|l|}{ aUG. } \\
\hline $01-06$ & 215 & 7.0 & - & 34 & 6.9 & 2.6 & 1.7 & 119 & 24 \\
\hline $07-16$ & 257 & 7.6 & - & 36 & $1 .-1$ & 2.5 & 1.8 & 116 & 25 \\
\hline $18-26$ & 234 & 8.0 & -- & 34 & 6.8 & 2.8 & 1.9 & 123 & 26 \\
\hline $25-31$ & 215 & 8.3 & - & 36 & 7.1 & 3.4 & 2.0 & 131 & 26 \\
\hline \multicolumn{10}{|l|}{ SEPT. } \\
\hline $01 . .$. & 215 & 6.3 & -- & 36 & 7.1 & 3.6 & 2.0 & 131 & 26 \\
\hline $02-05$ & 211 & 8.1 & - & 37 & 8.0 & 3.3 & 1.7 & 138 & 27 \\
\hline $06-19$ & 191 & 8.1 & - & 34 & 7.6 & 3.7 & 1.6 & 230 & 25 \\
\hline $20-30$ & [B] & 8.9 & -- & 32 & 7.6 & 3.1 & 1.4 & 123 & 23 \\
\hline
\end{tabular}

\begin{tabular}{|c|c|c|c|c|c|c|c|c|}
\hline DATE & $\begin{array}{l}\text { CHLO- } \\
\text { RIOE } \\
\text { CCLI }\end{array}$ & $\begin{array}{l}\text { FLUO- } \\
\text { RIOE } \\
\text { IF }\end{array}$ & $\begin{array}{l}\text { OIS- } \\
\text { SOLVEO } \\
\text { SOLIOS } \\
\text { ISUM OF } \\
\text { CONSII- } \\
\text { TUENTSI }\end{array}$ & $\begin{array}{l}\text { HARO- } \\
\text { NESS } \\
\text { ICA, MGI }\end{array}$ & $\begin{array}{l}\text { MON- } \\
\text { CAR- } \\
\text { BONATE } \\
\text { HARO- } \\
\text { MESS }\end{array}$ & $\begin{array}{l}\text { SPECI- } \\
\text { FIC } \\
\text { CONO- } \\
\text { UCTRNCE } \\
\text { IMICRO- } \\
\text { MHOSI }\end{array}$ & PH & COLOR \\
\hline \multicolumn{9}{|l|}{ ocr. } \\
\hline $01-05$ & .6 & .0 & 150 & 120 & 10 & 238 & 6.0 & 15 \\
\hline $06-14$ & 1.1 & -0 & 138 & 119 & 17 & 233 & 1.7 & 20 \\
\hline $15-17$ & 3.2 & -1 & 156 & 130 & 16 & 266 & 7.5 & is \\
\hline \multicolumn{9}{|l|}{ JUNE } \\
\hline $02 \ldots$ & 1.0 & .2 & 102 & 87 & 12 & 173 & 7.3 & 90 \\
\hline $03-17$ & 1.0 & .2 & 95 & 61 & 10 & 168 & 7.6 & 60 \\
\hline $18-27$ & -8 & -2 & 106 & 67 & 12 & 200 & 7.7 & 30 \\
\hline $28-30$ & 1.0 & .2 & 111 & 90 & 12 & 189 & 7.6 & 60 \\
\hline \multicolumn{9}{|l|}{ Jur } \\
\hline $01-02$ & 1.0 & -2 & 111 & 90 & 12 & 189 & 16.0 & 40 \\
\hline $08-11$ & .9 & .3 & 127 & 102 & 12 & 216 & 7.6 & 0 \\
\hline$|B-2|$ & 1.6 & -1 & 136 & 115 & 11 & 227 & 7.8 & 1 \\
\hline $28-31$ & 1.0 & -1 & 136 & 114 & 16 & 226 & 7.9 & 20 \\
\hline \multicolumn{9}{|l|}{ auc. } \\
\hline $01-06$ & 1.0 & .1 & 136 & 116 & 16 & 226 & 7.9 & 20 \\
\hline $07-16$ & 1.4 & .1 & 136 & 116 & 19 & 221 & 1.8 & 20 \\
\hline $18-24$ & .6 & -2 & 141 & 116 & 13 & 240 & 8.9 & 20 \\
\hline $25-31$ & 1.0 & .2 & 140 & 110 & 12 & 256 & 7.5 & 20 \\
\hline \multicolumn{9}{|l|}{ SEPT. } \\
\hline $01 \ldots$ & 1.0 & -2 & 169 & 119 & 12 & 254 & 7.5 & 20 \\
\hline $02-05$ & 1.4 & -1 & 155 & 126 & 13 & 262 & 1.8 & 10 \\
\hline $06-19$ & 1.8 & -2 & 167 & 121 & 14 & 253 & 7.6 & 15 \\
\hline $20-30$ & 1.0 & -1 & 137 & 111 & 10 & 230 & 1.5 & 20 \\
\hline
\end{tabular}


15-5648. YUKON RIVER AT RUBY--COntinued

SPECIFIC CONDUCTANCE (MICROMHOS AT $25^{\circ}$ C), MATER YEAR OCTOBER 1967 TO SEPTEMBER $196 B$

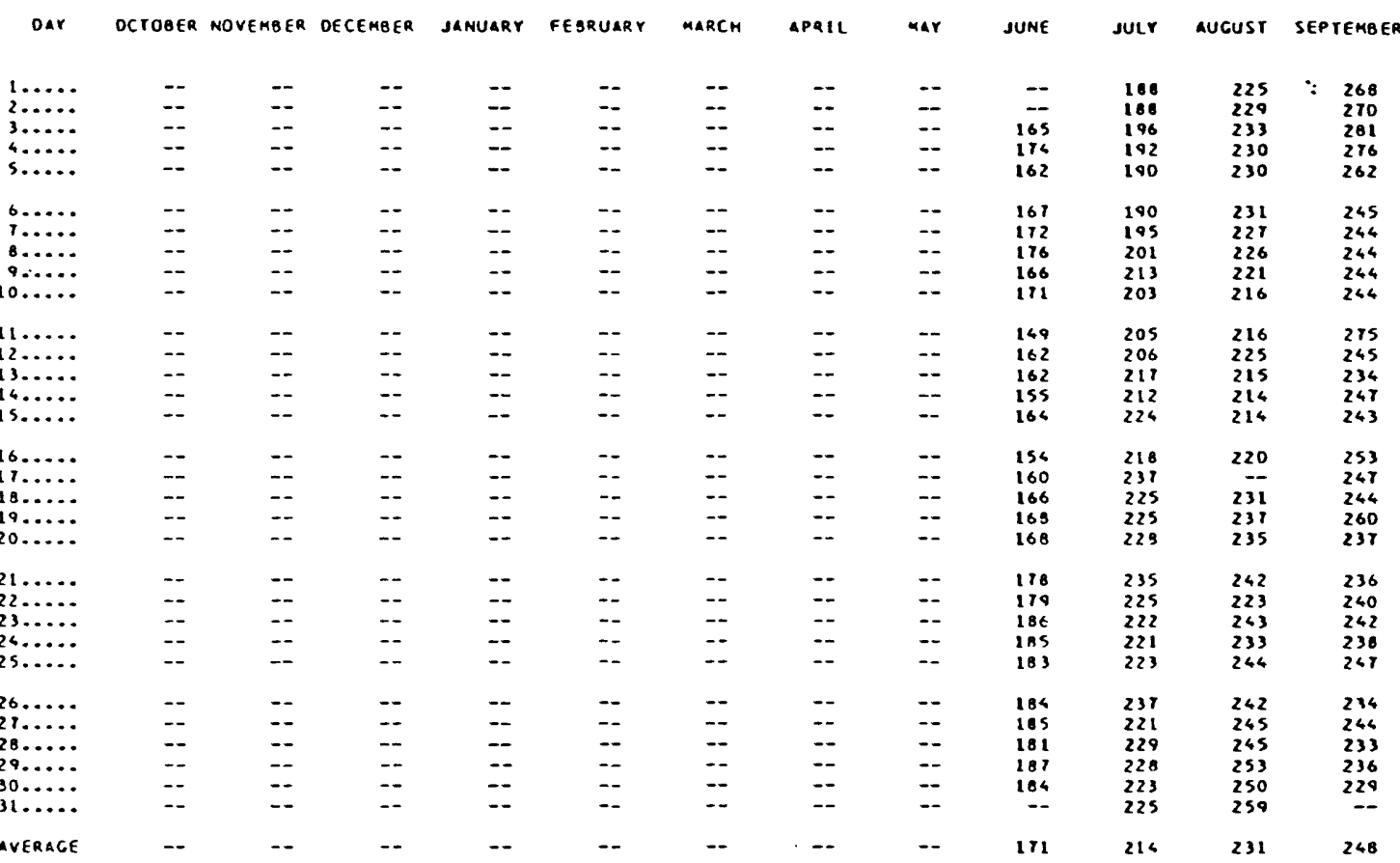

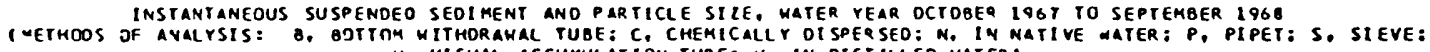
V. VISUAL aCCUMULAT ION TURE: W, IN DISTILLEO MATERI

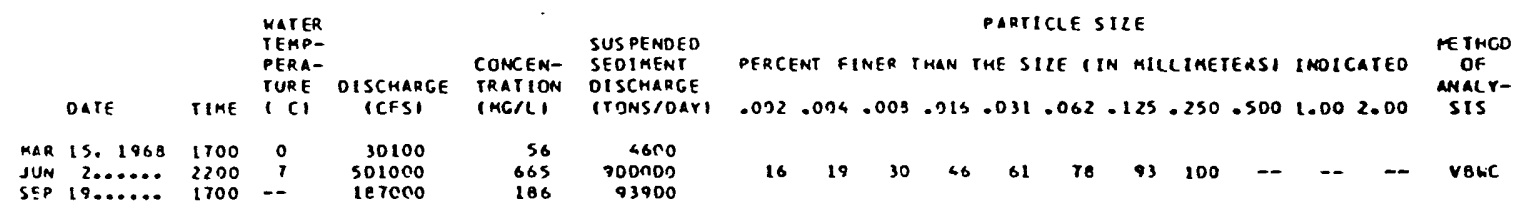


15-5618. YUKCN RI VER AT RUBY

(Internat1onal Hydrologic Decade Station)

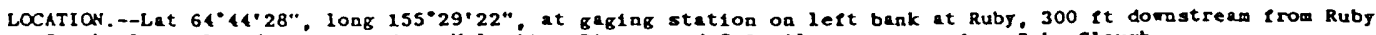
Creek, 1.5 mlles domstream from Melozitna River, and 2.2 miles upstream from Ruby Slough.

DRAINAGE AREA.--259,000 sq m1, epproximately.

PERIOD OF RECORD.-ChemlCal unalsses: June 1966 to september 1969.

Mater temperatures: June 1966 to September 1967 August to September 1969

Sedinent records: September 1967 to September 1969 (partial-record station).

EXTREXES, 1968-69.-Dissoived solids: Min1mum, 109 - $8 / 1$ June 8-19.

Hardness: Mnimum, $92 \mathrm{mg} / \mathrm{l}$ June $8-19$

Specific conductance: Malmum deily 185 micrombos June 7.

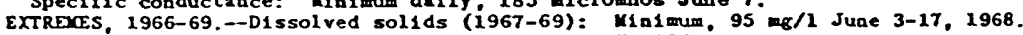

Hardness (1967-69): K1n1mum, 81 mo/1 June 13-17, 1968.

Specific conductance (1967-69): Minimum dally, ist merombos June 16, 1968.

Mater tenperatures (1966-67): Maximum, $18^{\circ} \mathrm{C}$ July 24, 26, 1966, June 24. July 11, 13, 1967.

RExurrs. - Streas f rozen over during perlod October to iay.

CHEKICAL ANALYSES, WATER YEAR OCTOBER 1968 TO SEPTEIKBER 1969

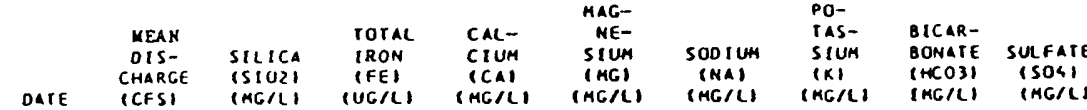

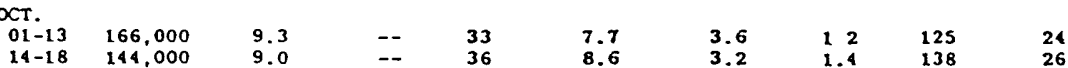

$14-18$

JN.

$08-19$
$20-30$

JUL.

$01-16$
$17-31$

$17-31$
AUG.

$01-04$
$08-17$

$08-17$
$18-27$

28-31

SEP.

254, 000

327,000

224,000

72,000

272.000

331.000

206,000

206.000

8.6

3.21 .4

$\begin{array}{lll}7.4 & .38 & 27 \\ 7.8 & 3.9 & 32\end{array}$

6.0
7.2

2.

$\begin{array}{lll}8.6 & 1.9 & 36 \\ \text { B. } 1 & 9.1 & 35\end{array}$

6.6
6.5

3.

$\begin{array}{rrr}8.1 & 9.1 & 35 \\ 6.1 & -- & 35 \\ 7.2 & -- & 32\end{array}$

$\begin{array}{ll}6.5 & 3.0 \\ 5.8 & 2.9 \\ 5.8 & 2.8 \\ 5.4 & 3.0\end{array}$

1.

07-30

188,000

$\begin{array}{lll}6.5 & .08 \quad 33\end{array}$

5.4
7.8

3.0
3.1

ANALYSES OF ADDITIONAL SAKPLES

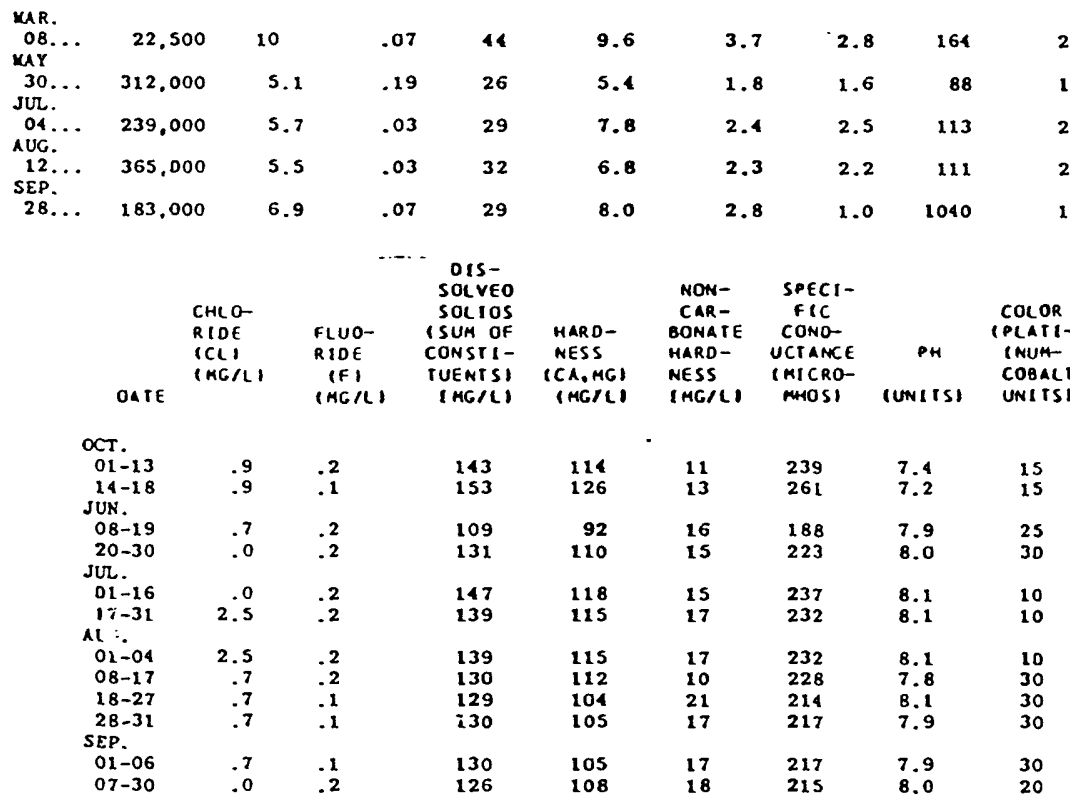

ANALYSES OF ADDITIONAL SAKPLES

\begin{tabular}{|c|c|c|}
\hline $\begin{array}{l}\text { MR. } \\
08 . \ldots\end{array}$ & .4 & .4 \\
\hline KAY & & \\
\hline $\begin{array}{l}30 . . \\
\text { JUL. }\end{array}$ & 1.1 & .2 \\
\hline $04 \ldots$ & .0 & .2 \\
\hline $\begin{array}{l}\text { AUC. } \\
12 . . \\
\text { SEP. }\end{array}$ & 1.1 & .1 \\
\hline $28 .$. & .4 & .1 \\
\hline
\end{tabular}

$\begin{array}{rr}178 & 149 \\ 102 & 88 \\ 126 & 105 \\ 129 & 109 \\ 124 & 105\end{array}$

$\begin{array}{rrrr}15 & 294 & 7.5 & 5 \\ 16 & 175 & 7.9 & 5 \\ 12 & 215 & 8.0 & -- \\ 18 & 223 & 8.0 & 30 \\ 19 & 215 & 8.0 & 30\end{array}$


15-5648. YUKON RIVER AT RUBT-COntiaued

SPECIFIC CONDUCTANCE (MICROMOHOS AT 23ㅇ). WATER YEAR OCTOBER 1968 TO SEPTEMBER 1969

\begin{tabular}{|c|c|c|c|c|c|c|c|c|c|c|c|c|}
\hline DAY & octoser & NOVERGER & DECDMBER & SANUARY & FEBRUARAY & WNRCH & APRIL & mr & JUNE & sulr & AUGUST & SEPTDKBE \\
\hline $1 \ldots$. & 230 & - & - & - & - & - & - & - & - & 230 & 226 & 216 \\
\hline $2 \ldots .$. & 227 & - & - & - & - & - & - & - & - & 247 & 221 & 219 \\
\hline 3..... & 233 & - & - & - & - & - & - & - & - & 230 & 229 & 210 \\
\hline $4 \ldots \ldots$ & 244 & - & - & - & - & - & - & - & - & 240 & 217 & 211 \\
\hline $3 . . .$. & 230 & - & - & - & - & - & - & 一 & - & 255 & - & 210 \\
\hline $6 \ldots$. & 237 & - & - & - & - & - & - & - & 186 & 248 & $\rightarrow$ & 213 \\
\hline $7 \ldots \ldots$ & 254 & - & - & - & - & $\overline{20}$ & - & 一 & 185 & 256 & - & 211 \\
\hline B..... & 249 & - & - & - & - & 249 & - & - & 166 & 248 & 292 & 213 \\
\hline $9 . . .$. & 240 & - & - & - & - & - & - & - & 189 & 240 & 279 & 213 \\
\hline $10 \ldots .$. & 241 & - & - & - & - & - & - & - & 192 & 238 & 228 & 217 \\
\hline $11 . . .$. & 240 & - & - & - & - & - & - & - & 190 & 240 & 217 & 219 \\
\hline $12 \ldots .$. & 240 & - & - & - & - & - & - & - & 186 & 238 & 217 & 217 \\
\hline $13 \ldots \ldots$ & 241 & - & - & - & - & - & - & - & 188 & 249 & 230 & 216 \\
\hline $14 \ldots \ldots$ & 252 & -- & - & - & - & - & - & - & 190 & 237 & 233 & 216 \\
\hline $15 \ldots . .$. & 258 & - & - & - & - & - & - & - & 194 & 241 & 210 & 216 \\
\hline $16 \ldots .$. & 262 & - & - & - & - & - & - & - & 194 & 251 & 200 & 219 \\
\hline $17 \ldots .$. & 267 & - & - & - & - & - & - & - & 198 & 239 & 203 & 217 \\
\hline $18 \ldots \ldots$ & 265 & -- & - & - & - & - & - & - & 188 & 239 & 220 & 215 \\
\hline $19 \ldots \ldots$ & - & - & - & - & - & - & - & - & 293 & 232 & 221 & 218 \\
\hline $20 \ldots$. & - & - & - & - & - & - & -- & - & 204 & 234 & 217 & 220 \\
\hline $21 \ldots \ldots$ & - & - & - & - & - & - & - & - & 222 & 229 & 215 & 219 \\
\hline $22 \ldots .$. & - & - & - & - & - & - & - & - & 216 & 225 & 215 & 217 \\
\hline $23 \ldots \ldots$ & - & - & - & - & - & - & - & - & 218 & 226 & 218 & 216 \\
\hline $24 \ldots .$. & -- & - & - & - & - & - & - & - & 225 & 226 & 216 & 216 \\
\hline $25 \ldots .$. & - & - & - & - & - & - & - & - & 229 & 260 & 218 & 217 \\
\hline $26 \ldots \ldots$ & - & - & - & - & - & - & - & - & 234 & 223 & 214 & 218 \\
\hline $27 \ldots \ldots$ & - & - & - & - & - & - & - & - & 234 & 222 & 215 & 220 \\
\hline $28 \ldots .$. & - & - & - & - & - & - & - & $\rightarrow$ & 231 & 216 & 218 & 217 \\
\hline $29 \ldots \ldots$ & - & - & - & - & - & - & - & - & 231 & 223 & 216 & 217 \\
\hline $30 \ldots .$. & - & - & - & - & - & - & - & - & 238 & 219 & 223 & 222 \\
\hline $31 \ldots \ldots$ & - & - & - & - & - & - & - & - & - & 232 & 217 & - \\
\hline AVERACE & - & - & - & - & - & - & - & - & 206 & 237 & 223 & 216 \\
\hline
\end{tabular}

TELPERATURE ('C) OF WATER, AUGUST TO SEPTEMBER 1969

oar

MONTH $123456 \quad 7.91011 \quad 1213141516171819202122 \quad 23242526 \quad 2728293031$ AGE

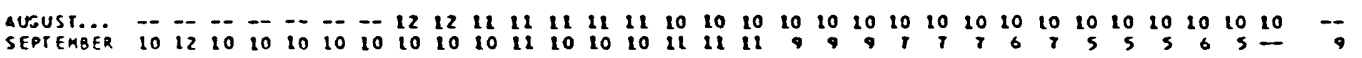

INSTANTANEOUS SUSPENDEO SEOIKENT ANO PARTICLE SIZE, MATER VEAR OCTOBER 1960 TO SEPTENBER 1969

INETHOOS OF ANALYSIS: B. DOTTOM WITHORAKM TUBE: C. CHENICALCY DISPERSEO: N. IN MATIVE WATER: P. PIPET: S. SIEVE: V. VISUAL ACCUAUCATION TUBE: N. IN OISTILLEO WATERI

\begin{tabular}{|c|c|c|c|c|c|c|c|c|c|c|c|c|c|c|c|c|c|c|c|}
\hline \multicolumn{2}{|r|}{ DATE } & \multirow{2}{*}{$\begin{array}{l}\text { TIHE } \\
100 T\end{array}$} & $\begin{array}{l}\text { MATER } \\
\text { TEMP- } \\
\text { PERA- } \\
\text { TURE } \\
\text { I CI }\end{array}$ & $\begin{array}{l}\text { DISCHARGE } \\
\text { (CFSI) }\end{array}$ & $\begin{array}{l}\text { CONCEN- } \\
\text { TRATION } \\
\text { (NGIL) }\end{array}$ & \multirow{2}{*}{$\begin{array}{c}\text { SUSPENOEO } \\
\text { SEOIAENT } \\
\text { OISCHARGE } \\
\text { ITONSIOAYI } \\
119\end{array}$} & $\begin{array}{l}\text { PERCEN } \\
.002\end{array}$ & & THAN & 5.031 & TICLE & $E 518$ & .125 & .250 & .500 & 1.00 & 2.00 & $\begin{array}{l}\text { KETHOD } \\
\text { Of } \\
\text { ANALY- } \\
\text { SIS }\end{array}$ \\
\hline MAR & B. 1969 & & 0.0 & 22100 & 2 & & - & - & - & -- & -- & & -- & - & - & - & $\rightarrow$ & - & \\
\hline mar & $30 \ldots \ldots$ & 1130 & 12.0 & 313000 & 545 & 461000 & 5 & 9 & 16 & 20 & 42 & & 50 & 81 & 99 & 100 & - & $\cdots$ & vesu \\
\hline sut & $4 \ldots \ldots$ & 2300 & 16.0 & 239000 & 772 & 498000 & 37 & so & 54 & 65 & 10 & & 76 & BS & 95 & 100 & -- & $\cdots$ & VCPW \\
\hline AUG & $13 \ldots \ldots$ & 1100 & 9.0 & 366000 & 867 & 857000 & 17 & 24 & 35 & si & 67 & & Tt & 08 & 100 & -- & - & - & VCPN \\
\hline SEP & $20 \ldots \ldots$ & 1130 & & 184000 & 132 & 65600 & - & - & - & - & -- & & 38 & 66 & 100 & -- & -- & - & vw \\
\hline
\end{tabular}


LOCATION. - Lat $64^{\circ} 44^{\circ} 28^{\prime \prime}$. LOng $155^{\circ} 29^{\circ} 22^{*}$, at gaging station on left bank at Ruby. 300 ft downstream from Ruby Creek. : 5 miles domstream from Melozitna River, and 2.5 miles upstream from Ruby Slough.

DRAINAGE IREA. - 259.000 sq mi. approxinately.

PERIOD OF RECORD. - Chemical analyses: June 1966 to September 1970. Wacer temperatures: June 1966 to September 1967. August 1969 to September 1970. Sediment records: September 1967 to September 1970 (partial-record station).

EXTREMES, 1969-70, . Dissolved solids: Maximum. $169 \mathrm{mg} / 1 \mathrm{Mar} .8$ : minimum, $92 \mathrm{mg} / \mathrm{l}$ June 12. Hardness: Maximum. $140 \mathrm{mg} / 1 \mathrm{Mar}$. B: minimum. $80 \mathrm{mg} / 1$ June 12. Specific conductance: Maximua, 280 micromhos Mar. 8: minimum daily, 261 micromhos June 12. Water temperatures: Maximum, is ${ }^{\circ} \mathrm{C}$ July 1. 2, 4-6.

EXTRENES, 1966-70: Dissolved solids (1967-70): Maximum, 169 mg/1 Mar. 8, 1970; minimum. B7mg/1 May 22-31, 1967. Hardness $(1967-70):$ Maximum, $140 \mathrm{mg} / 1$ Mar. 8, 2970 : minimum, is mg/1 May $22-31,1967$.

Specific conductance (1967-jo): Maximum, 315 micromos Aug. 1-16, 1967: minimum, 151 micromhos

May $22-31,1967$.

Water temperatures (1966, 1967, 1970): Maximum, $18^{\circ} \mathrm{C}$ July 21, 26, 1966, June 24, July 11, $13,1967$.

July 1.2, 4-6. 1970 .

REMARKS. - River frozen over during period October to May. Miscellaneous chemical data published for water years $1967-70$ and sediment data for water years $1967-70$.

CHEKICAL ANACYSES, wateR YEAR OCTOBER 1969 10 SEPTEKOER 1970

\begin{tabular}{|c|c|c|c|c|c|c|c|c|c|c|}
\hline DATE & $\begin{array}{l}\text { CIS- } \\
\text { CHARGE } \\
\text { ICFSI }\end{array}$ & $\begin{array}{l}\text { SILICA } \\
\text { ISIOLI) } \\
\text { IMC/LI }\end{array}$ & $\begin{array}{l}\text { TOTAL } \\
\text { IRCN } \\
\text { IFEI } \\
\text { IUGIL }\end{array}$ & $\begin{array}{l}\text { OIS- } \\
\text { SOLVEO } \\
\text { IRON } \\
\text { IFEI } \\
\text { CUGALI }\end{array}$ & $\begin{array}{l}\text { CAL- } \\
\text { CIUA } \\
\text { CCAI } \\
\text { CAGIL) }\end{array}$ & $\begin{array}{l}\text { MAC- } \\
\text { NE- } \\
\text { SIUK } \\
\text { INGI } \\
\text { INGRLI }\end{array}$ & $\begin{array}{l}\text { SootuK } \\
\text { (NRI) } \\
\text { ING/LI }\end{array}$ & $\begin{array}{l}\text { PO- } \\
\text { rAS- } \\
\text { SIUN } \\
\text { IKI } \\
\text { IAGACI }\end{array}$ & $\begin{array}{l}\text { OTCAR- } \\
\text { OOMATE } \\
\text { IHCOBI } \\
\text { CMGRLI }\end{array}$ & $\begin{array}{l}\text { SULFATE } \\
\text { (SOAI } \\
\text { INGIL) }\end{array}$ \\
\hline $\begin{array}{l}\text { MAY } \\
25-31 \\
\text { JUAE }\end{array}$ & $300 c 00$ & 1.1 & -- & 20 & 26 & 5.3 & 2.1 & 1.2 & 85 & 18 \\
\hline $\begin{array}{l}01-30 \\
\text { suer }\end{array}$ & 362000 & 7.1 & - & 20 & 26 & 5.3 & 2.1 & 1.2 & os & 18 \\
\hline $\begin{array}{l}01-02 \\
03-23 \\
24-31\end{array}$ & $\begin{array}{l}362000 \\
340000 \\
311000\end{array}$ & $\begin{array}{l}7.1 \\
3.1 \\
6.4\end{array}$ & $\begin{array}{l}20 \\
\text { so }\end{array}$ & $\begin{array}{l}20 \\
50\end{array}$ & $\begin{array}{l}26 \\
26 \\
30\end{array}$ & $\begin{array}{l}5.3 \\
6.2 \\
1.3\end{array}$ & $\begin{array}{l}2.1 \\
2.4 \\
3.2\end{array}$ & $\begin{array}{l}1.2 \\
1.5 \\
1.5\end{array}$ & $\begin{array}{r}85 \\
93 \\
110\end{array}$ & $\begin{array}{l}18 \\
15 \\
22\end{array}$ \\
\hline $\begin{array}{l}\text { AUG. } \\
C 1-31 \\
\text { SEPI. } \\
01-21\end{array}$ & 311000 & 6.4 & 50 & so & 30 & $\begin{array}{l}7.3 \\
7.3\end{array}$ & 3.2 & 1.5 & 110 & 22 \\
\hline & & & ANAL & SES of & LOOTLONAL & SAKPLES & & & & \\
\hline CAR.... & 27200 & 10 & - & so & 42 & 0.6 & 3.6 & 2.2 & 152 & 23 \\
\hline $\begin{array}{l}\text { SLAE } \\
12 \ldots \\
\text { JuLr }\end{array}$ & 331000 & 4.8 & -- & -- & 24 & 4.8 & 1.8 & 1.3 & 70 & 16 \\
\hline $\begin{array}{l}12 \ldots \\
\text { AUG. }\end{array}$ & 317000 & 7.1 & 120 & -- & 27 & 5.7 & 2.2 & 1.4 & 87 & 21 \\
\hline $\begin{array}{l}15 \ldots . \\
\text { SEPT. }\end{array}$ & 262000 & 5.6 & - & 60 & 33 & 6.6 & 2.2 & 1.3 & 110 & 22 \\
\hline $21 \ldots$ & 260000 & 6.0 & -- & 160 & 34 & 7.5 & 2.7 & 1.1 & 114 & 23 \\
\hline
\end{tabular}


15564800 YUKCS RIVER AT RUBY--CONtinued

CHEMICAL ANALYSES, KATER YEAR OCTOBER 1969 TO SEFTEMBER 1970--Continued

\begin{tabular}{|c|c|c|c|c|c|c|c|c|c|c|}
\hline OATE & $\begin{array}{l}\text { CHLO- } \\
\text { RIOE } \\
\text { ICLI } \\
\text { IMG/LI }\end{array}$ & $\begin{array}{l}\text { FLUO- } \\
\text { RICE } \\
(F \mid \\
(K C, / C)\end{array}$ & $\begin{array}{l}\text { NITRATE } \\
\text { IACBI } \\
\text { INGILI }\end{array}$ & $\begin{array}{l}\text { OIS- } \\
\text { SOLVEO } \\
\text { SCLIOS } \\
\text { ISUM OF } \\
\text { CONSTI- } \\
\text { TUENTS: } \\
\text { IMGICI }\end{array}$ & $\begin{array}{l}\text { HARO- } \\
\text { NESS } \\
\text { ICA.MGI } \\
\text { (MG/LI }\end{array}$ & $\begin{array}{l}\text { NON- } \\
\text { CAR- } \\
\text { OONATE } \\
\text { MARD- } \\
\text { NESS } \\
\text { IMG/LI }\end{array}$ & $\begin{array}{l}\text { SPECI- } \\
\text { FIC } \\
\text { COND- } \\
\text { UCTANCE } \\
\text { IMICRO- } \\
\text { MHOSI }\end{array}$ & $\begin{array}{c}P H \\
\text { IUNITSI }\end{array}$ & $\begin{array}{l}\text { COLOR } \\
\text { IPLAT- } \\
\text { INUA- } \\
\text { COBALT } \\
\text { UNITSI }\end{array}$ & $\begin{array}{l}\text { TEKP- } \\
\text { ERATURE } \\
\text { IOEG CI }\end{array}$ \\
\hline rar & & & & & & & & & & \\
\hline $\begin{array}{l}25-31 \\
\text { JUNE }\end{array}$ & 1.2 & .2 & .2 & 103 & 86 & 16 & 176 & 7.8 & 20 & -- \\
\hline $\begin{array}{l}\text { ol-30 } \\
\text { Juer }\end{array}$ & 1.2 & .2 & .2 & 103 & 86 & 16 & 176 & 7.8 & 20 & $\cdots$ \\
\hline $01-02$ & 1.2 & .2 & -2 & 103 & 66 & 16 & 176 & 7.8 & 20 & - \\
\hline $\begin{array}{l}03-23 \\
24-31\end{array}$ & $\begin{array}{l}1.5 \\
.8\end{array}$ & .2 & 1.4 & 103 & $\begin{array}{r}91 \\
105\end{array}$ & $\begin{array}{l}15 \\
15\end{array}$ & $\begin{array}{l}194 \\
219\end{array}$ & 7.5 & 25 & $=$ \\
\hline $\begin{array}{l}\text { AUC. } \\
\text { O1-31 } \\
\text { SEPT. }\end{array}$ & .8 & .2 & .3 & 126 & 105 & 25 & 210 & 0.1 & 10 & - \\
\hline $01-21$ & -3 & .2 & $\cdot 3$ & 126 & 105 & 15 & 219 & 8.1 & 10 & $\cdots$ \\
\hline \multicolumn{11}{|c|}{ ANALYSES OF AOOITIONAL SAMPLES } \\
\hline $\begin{array}{l}\text { PAR. } \\
\text { OB... }\end{array}$ & 1.4 & .2 & .8 & 169 & 140 & is & 280 & 0.2 & 10 & .0 \\
\hline $\begin{array}{l}\text { JUAE } \\
\text { L2... } \\
\text { JUEr }\end{array}$ & .0 & .2 & .8 & 92 & 80 & 16 & 161 & 7.7 & 15 & 13.5 \\
\hline $\begin{array}{c}12 . . . \\
\text { auc. }\end{array}$ & .1 & .1 & 1.0 & 108 & 91 & 20 & 186 & 7.9 & so & 11.5 \\
\hline $\begin{array}{l}\text { IS... } \\
\text { SEPI. }\end{array}$ & .5 & -1 & .2 & 126 & 111 & 21 & 218 & 7.7 & 10 & 15.5 \\
\hline $21 \ldots$ & 1.0 & -1 & .4 & 132 & 117 & 24 & 225 & 7.6 & 20 & 3.0 \\
\hline
\end{tabular}

SPECIFIC CONDUCTANCE (MICROMOHOS AT $25^{\circ}$ C), WATER YEAR OCTOBER 1969 TO SEPTEMBER 1970

\begin{tabular}{|c|c|c|c|c|c|c|c|c|c|c|c|c|}
\hline our & CCTOBER & NOVEMBER & DECDABER & JANUARY & FEBRUNRY & MURCH & APRIL. & mar & JUNE & JuLY & AUCUST & SEPTEMBER \\
\hline $1 \ldots$ & 225 & - & - & -- & -- & - & - & -- & 181 & 178 & 211 & 208 \\
\hline $2 \ldots$ & 223 & - . & - & - & - & - & - & - & 175 & 183 & 211 & 211 \\
\hline $3 \ldots$. & 225 & -- & -- & - & - & - & - & -- & 165 & 191 & 219 & 209 \\
\hline $4 \ldots$. & 229 & - & - & - & - & - & - & - & 166 & 190 & 212 & 233 \\
\hline$s, \ldots$. & 250 & -- & - & -- & - & - & - & - & 169 & 197 & 208 & 230 \\
\hline $6 \ldots .$. & 235 & - & - & - & - & - & - & - & 159 & 187 & 217 & 236 \\
\hline $7 \ldots$. & - & -- & - & -- & - & - & - & -- & 160 & 186 & 214 & 221 \\
\hline 8...... & - & - & - & - & - & 294 & - & - & 158 & 186 & 213 & 220 \\
\hline $9 . \ldots$ & - & - & - & - & - & - & - & - & 163 & 190 & 216 & 220 \\
\hline $10 \ldots .$. & - & - & - & - & - & - & - & - & 262 & 186 & 234 & 222 \\
\hline $11 \ldots .$. & -- & -- & - & - & - & - & - & - & 168 & 189 & 237 & 220 \\
\hline $12 \ldots \ldots$ & -- & - & - & - & - & - & - & - & 164 & 208 & 240 & 222 \\
\hline $13 \ldots \ldots$ & - & -- & - & - & - & - & - & - & 161 & 188 & 239 & 222 \\
\hline $14 . . .$. & -- & - & -- & - & -- & - & - & - & 161 & 190 & 216 & 224 \\
\hline $15 . . .$. & -- & -- & - & - & - & - & - & - & 167 & 206 & 219 & 224 \\
\hline $16, \ldots \ldots$ & -- & - & - & -- & - & - & - & - & 175 & 190 & 217 & 223 \\
\hline $17 \ldots .$. & -- & -- & -- & - & - & -- & - & - & 175 & 192 & 215 & 222 \\
\hline $18 \ldots .$. & -- & -- & -- & -- & - & - & - & - & 181 & 195 & 210 & 234 \\
\hline $19 . \ldots$. & - & - & - & -- & -- & - & - & - & 160 & 203 & 210 & 234 \\
\hline $20 . . .$. & -- & -- & - & - & - & - & - & - & 181 & 194 & 217 & 235 \\
\hline $21 \ldots$. & -- & -- & -- & - & - & - & - & - & 182 & 197 & 217 & 228 \\
\hline $22 \ldots .$. & - & -- & -- & -- & - & - & - & - & 184 & 195 & 230 & - \\
\hline $23 . .$. & - & -- & -- & -- & -- & - & - & - & 179 & 209 & 228. & - \\
\hline $24 . . .$. & -- & -- & -- & - & - & - & -- & - & 174 & 235 & 228 & - \\
\hline $25 \ldots .$. & -- & - & -- & -- & -- & - & -- & 181 & 175 & 212 & 209 & - \\
\hline $26 \ldots .$. & - & -- & -- & - & - & - & - & 183 & 185 & 215 & 205 & - \\
\hline $27 \ldots .$. & - & - & - & -- & - & - & - & 183 & 186 & 213 & 207 & - \\
\hline $28 \ldots \ldots$ & - & -- & -- & - & - & - & - & 183 & 162 & 211 & 212 & - \\
\hline $29 . \ldots$. & - & -- & - & -- & - & - & - & 185 & 181 & 207 & 206 & - \\
\hline $30 \ldots . .$. & - & - & - & -- & - & - & - & 164 & 179 & 208 & 210 & - \\
\hline $31 \ldots$. & $-\cdot$ & $\cdots$ & - & - & - & - & - & 179 & - & 209 & 216 & - \\
\hline IVERACE & -- & -- & - & - & - & - & - & - & 173 & 198 & 216 & - \\
\hline
\end{tabular}


15564800 YUKON RIVER AT RUBY--COntinued

WATER QUALITY DATA. WATER YEAR OCTOBER 1970 TO SEPTEMGER 1971 --CONTINUEd

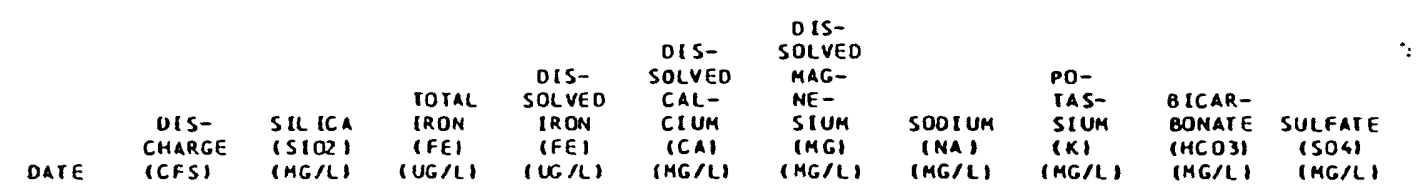

\begin{tabular}{|c|c|c|c|c|c|c|c|c|c|c|}
\hline $\begin{array}{l}\text { MAY } \\
3 t-3 t \\
\text { JUNE }\end{array}$ & 636800 & 4.2 & - & -- & 22 & 4.0 & 1.9 & 1.3 & 78 & 9.8 \\
\hline $\begin{array}{l}01-14 \\
15-29 \\
30-30\end{array}$ & $\begin{array}{l}636800 \\
433000 \\
324400\end{array}$ & $\begin{array}{l}4.2 \\
5.3 \\
5.7\end{array}$ & -- & $\begin{array}{l}-- \\
-- \\
--\end{array}$ & $\begin{array}{l}22 \\
26 \\
30\end{array}$ & $\begin{array}{l}4.0 \\
5.1 \\
6.1\end{array}$ & $\begin{array}{l}1.9 \\
2.3 \\
2.8\end{array}$ & $\begin{array}{l}1.3 \\
1.5 \\
1.5\end{array}$ & $\begin{array}{r}78 \\
96 \\
107\end{array}$ & $\begin{array}{l}9.8 \\
13 \\
19\end{array}$ \\
\hline $\begin{array}{l}\text { SULY } \\
01-17 \\
18-31 \\
\text { AUG. }\end{array}$ & $\begin{array}{l}324400 \\
312500\end{array}$ & $\begin{array}{l}5.7 \\
6.1\end{array}$ & -- & -- & $\begin{array}{l}30 \\
36\end{array}$ & $\begin{array}{l}6.1 \\
6.4\end{array}$ & $\begin{array}{l}2.8 \\
3.2\end{array}$ & $\begin{array}{l}1.5 \\
1.9\end{array}$ & $\begin{array}{l}107 \\
126\end{array}$ & $\begin{array}{l}19 \\
19\end{array}$ \\
\hline $\begin{array}{l}01-28 \\
29-31 \\
\text { SEP. } \\
01-29\end{array}$ & $\begin{array}{l}312500 \\
224400\end{array}$ & $\begin{array}{l}6.1 \\
6.8\end{array}$ & -- & -- & $\begin{array}{l}36 \\
33\end{array}$ & $\begin{array}{l}6.4 \\
7.3\end{array}$ & $\begin{array}{l}3.2 \\
3.7\end{array}$ & $\begin{array}{l}1.9 \\
1.5 \\
1.5\end{array}$ & $\begin{array}{l}126 \\
117\end{array}$ & $\begin{array}{l}19 \\
22 \\
22\end{array}$ \\
\hline
\end{tabular}

ANAL YSES DF AODT TIONAL SAMPLES

\begin{tabular}{|c|c|c|c|c|c|c|c|c|c|c|}
\hline $\begin{array}{l}\text { MAR. } \\
\text { I9... } \\
\text { JUNE }\end{array}$ & 26500 & 10 & -- & 180 & 42 & 9.7 & 4.0 & 1.6 & 154 & 24 \\
\hline $\begin{array}{l}09 . . . \\
\text { suty }\end{array}$ & 594000 & 6.1 & -- & 40 & 22 & 3.7 & 1.2 & 1.1 & 76 & 9.6 \\
\hline $\begin{array}{l}14 \ldots \\
\text { AUG. }\end{array}$ & 274000 & 5.6 & 2700 & -- & 32 & 6.3 & 2.3 & 1.2 & 106 & 20 \\
\hline
\end{tabular}

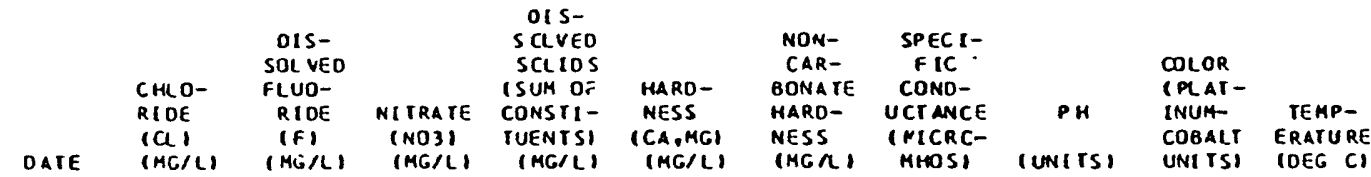

$\begin{array}{lll}\text { MAY } & \\ 31-31 & .5 & .3 \\ \text { JUNE } & & \\ 01-16 & .5 & .3 \\ 15-29 & .5 & .2 \\ 30-30 & .5 & .1 \\ \text { JUI } & & \\ 01-17 & .5 & .1 \\ 18-31 & 1.0 & .2 \\ \text { AUG. } & 1.0 & .2 \\ 01-28 & 1.0 & .2 \\ 29-31 & & .2 \\ \text { SEP. } & & \\ 01-29 & 1.0 & .2\end{array}$

$\begin{array}{rrrrrrrr}.7 & 13 & 72 & 8 & 142 & 7.6 & 50 & -- \\ .7 & 83 & 72 & 8 & 142 & 7.6 & 50 & -- \\ .2 & 101 & 86 & 7 & 179 & 7.5 & 20 & - \\ .1 & 119 & 100 & 12 & 206 & 8.1 & 10 & - \\ .1 & 119 & 100 & 12 & 206 & 8.1 & 10 & -- \\ .1 & 136 & 117 & 14 & 238 & 7.4 & 40 & -- \\ .1 & 136 & 117 & 14 & 238 & 7.4 & 40 & -- \\ .1 & 134 & 113 & 17 & 232 & 7.6 & 10 & - \\ .1 & 134 & 113 & 17 & 232 & 7.6 & 10 & --\end{array}$

ANALYSES OF AODITIONAL SAMPLES

\begin{tabular}{|c|c|c|c|c|c|c|c|c|c|c|}
\hline IQ... & 1.0 & .1 & .6 & 169 & 146 & 20 & 287 & 7.8 & 10 & .0 \\
\hline O9... & 2.0 & .2 & .4 & 83 & 73 & 11 & 142 & 7.6 & 50 & 13.0 \\
\hline $\begin{array}{l}14 . . \\
\text { AUG. }\end{array}$ & .5 & .2 & .0 & 120 & 106 & 19 & 207 & 7.7 & 10 & - \\
\hline $15 \ldots$ & .5 & .2 & .1 & 133 & 115 & 17 & 229 & 7.7 & 30 & 13.0 \\
\hline
\end{tabular}


15564800 YUKON RIVER AT RUBY--COntínued

TEMPERATURE $\left({ }^{\circ} \mathrm{C}\right)$ OF WATER, WATER YEAR OCTOBER 1970 TO SEPTEMBER 1971

\begin{tabular}{|c|c|c|c|c|c|c|c|c|c|c|c|c|}
\hline CAY & CCT & NCV & CEC & JAA & FEE & MAR & $\triangle P R$ & MAY & JUN & JUL & AUG & SEP \\
\hline $\begin{array}{l}1 \\
2 \\
3 \\
4 \\
5\end{array}$ & $\begin{array}{l}-- \\
-- \\
-- \\
--\end{array}$ & $\overline{-}$ & $\underline{E}$ & $\underline{--}$ & $\overline{-}$ & $\begin{array}{l}-\overline{-} \\
-\overline{-} \\
-\overline{-}\end{array}$ & $\overline{-}$ & $\begin{array}{l}-- \\
-- \\
--\end{array}$ & $\begin{array}{r}8.0 \\
8.0 \\
9.0 \\
9.0 \\
10.0\end{array}$ & $\begin{array}{l}17.0 \\
16.0 \\
16.0 \\
15.5 \\
15.0\end{array}$ & $\begin{array}{l}14.5 \\
14.5 \\
14.0 \\
14.5 \\
15.0\end{array}$ & $\begin{array}{l}13.0 \\
11.5 \\
11.5 \\
11.0 \\
10.0\end{array}$ \\
\hline $\begin{array}{r}6 \\
7 \\
9 \\
10\end{array}$ & $\overline{-}$ & $\overline{--}$ & $\underline{E}$ & $\overline{--}$ & $\begin{array}{l}-- \\
-\square \\
--\end{array}$ & $\overline{-}$ & $=$ & $\bar{E}$ & $\begin{array}{l}10.0 \\
11.0 \\
12.0 \\
13.0 \\
14.0\end{array}$ & $\begin{array}{l}15.5 \\
15.5 \\
16.0 \\
17.0 \\
17.0\end{array}$ & $\begin{array}{l}14.5 \\
14.5 \\
14.0 \\
14.0 \\
14.5\end{array}$ & $\begin{array}{l}9.5 \\
9.0 \\
8.5 \\
8.5 \\
8.5\end{array}$ \\
\hline $\begin{array}{l}11 \\
12 \\
13 \\
14 \\
15\end{array}$ & $\begin{array}{l}-- \\
--- \\
--- \\
--\end{array}$ & $\begin{array}{l}--- \\
--- \\
--- \\
---\end{array}$ & $\underline{E}$ & $\begin{array}{l}-- \\
-- \\
--\end{array}$ & $\begin{array}{l}-- \\
-- \\
--\end{array}$ & $\overline{-}$ & $=$ & $\begin{array}{l}-- \\
-- \\
--\end{array}$ & $\begin{array}{l}14.0 \\
15.5 \\
15.5 \\
14.0 \\
14.5\end{array}$ & $\begin{array}{l}17.0 \\
17.5 \\
16.5 \\
15.5 \\
15.5\end{array}$ & $\begin{array}{l}14.0 \\
14.5 \\
15.5 \\
15.0 \\
15.0\end{array}$ & $\begin{array}{l}8.5 \\
8.0 \\
8.0 \\
8.0 \\
7.5\end{array}$ \\
\hline $\begin{array}{l}16 \\
17 \\
18 \\
19 \\
20\end{array}$ & 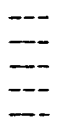 & $\overline{--}$ & $\bar{z}$ & $\begin{array}{l}-\cdots \\
-\cdots \\
--- \\
--\end{array}$ & $\begin{array}{l}-- \\
-- \\
-- \\
--\end{array}$ & $\begin{array}{l}-- \\
-- \\
--\end{array}$ & $=$ & $\begin{array}{l}-- \\
-\cdots \\
-- \\
--\end{array}$ & $\begin{array}{l}13.0 \\
13.0 \\
13.0 \\
14.0 \\
15.0\end{array}$ & $\begin{array}{l}15.0 \\
15.5 \\
16.5 \\
16.5 \\
16.5\end{array}$ & $\begin{array}{l}14.0 \\
14.0 \\
14.0 \\
14.0 \\
12.0\end{array}$ & $\begin{array}{l}7.0 \\
7.0 \\
7.0 \\
6.5 \\
6.0\end{array}$ \\
\hline $\begin{array}{l}21 \\
22 \\
23 \\
24 \\
25\end{array}$ & $=$ & $\overline{-}$ & $\bar{z}$ & $\begin{array}{l}\cdots- \\
\cdots \\
\cdots- \\
--\end{array}$ & $\begin{array}{l}--- \\
--- \\
--- \\
---\end{array}$ & $\begin{array}{l}-- \\
-- \\
-\end{array}$ & -- & $\begin{array}{l}-- \\
-- \\
--\end{array}$ & $\begin{array}{l}16.0 \\
17.0 \\
17.0 \\
17.5 \\
18.0\end{array}$ & $\begin{array}{l}16.5 \\
16.5 \\
17.0 \\
17.0 \\
17.0\end{array}$ & $\begin{array}{l}13.0 \\
12.5 \\
12.5 \\
12.0\end{array}$ & $\begin{array}{l}7.0 \\
7.0 \\
7.0 \\
7.0 \\
6.0\end{array}$ \\
\hline $\begin{array}{l}26 \\
27 \\
28 \\
28 \\
30 \\
31\end{array}$ & $\begin{array}{l}--- \\
-- \\
-- \\
--\end{array}$ & $\begin{array}{l}-- \\
-- \\
-- \\
-- \\
--\end{array}$ & $\overline{-}$ & $\begin{array}{l}-- \\
--- \\
-- \\
-- \\
--\end{array}$ & $\overline{-}$ & $\begin{array}{l}--- \\
-- \\
-- \\
-- \\
--\end{array}$ & $\overline{-}$ & $\begin{array}{c}-- \\
-- \\
-- \\
-\overline{0.0}\end{array}$ & $\begin{array}{r}18.5 \\
18.5 \\
18.5 \\
17.5 \\
- \\
\end{array}$ & $\begin{array}{r}16.5 \\
17.0 \\
16.0 \\
16.0 \\
15.5\end{array}$ & $\begin{array}{l}12.0 \\
13.0 \\
13.0 \\
13.0 \\
13.0 \\
13.0\end{array}$ & $\begin{array}{l}5.5 \\
5.0 \\
4.5 \\
4.5 \\
5.0 \\
\end{array}$ \\
\hline DKTH & -- & -- & -- & $-\cdots$ & -- & -- & -- & -- & 14.0 & 16.5 & 14.0 & 6.0 \\
\hline
\end{tabular}

SUSPENOED SEOIMENT AMALYSES. NATER YEAR OCTOBER 1970 TO SEPTEMBER I97I

\begin{tabular}{|c|c|c|c|c|c|c|c|c|c|}
\hline & TIME & $\begin{array}{l}\text { TEMP- } \\
\text { ERATURE }\end{array}$ & $\begin{array}{l}\text { SPECI- } \\
\text { FIC } \\
\text { CONO- } \\
\text { UCT ANCE } \\
\text { IMICRO- }\end{array}$ & $\begin{array}{l}\text { TUR- } \\
\text { BIO- } \\
\text { IIY }\end{array}$ & DIS- & $\begin{array}{l}\text { SUS- } \\
\text { PENOEO } \\
\text { SEOI- } \\
\text { MENT }\end{array}$ & $\begin{array}{l}\text { SUS- } \\
\text { PENDEO } \\
\text { SEOI- } \\
\text { KENT } \\
\text { OIS- } \\
\text { CHARGE }\end{array}$ & $\begin{array}{l}\text { SUS } \\
\text { SEO } \\
\text { FAL } \\
\text { OIAH } \\
\text { T FIN } \\
\text { THA }\end{array}$ & LER \\
\hline DATE & & (OEG C) & MHOSI & (stu) & |CFSI & $(\mathrm{MG} / \mathrm{L})$ & (T/OAY) & .002 & MH \\
\hline $\begin{array}{l}\text { MAR. } \\
\text { I9... } \\
\text { JUNE }\end{array}$ & 1915 & .0 & 300 & 一 & 26500 & 16 & 1150 & & -- \\
\hline $\begin{array}{c}09 . . . \\
\text { AUG. } \\
15 \ldots . .\end{array}$ & 1815 & 13.0 & 142 & 60 & 584000 & 518 & 831000 & & 11 \\
\hline
\end{tabular}

\begin{tabular}{|c|c|c|c|c|c|c|c|c|c|c|c|c|c|c|c|c|}
\hline \multirow[b]{3}{*}{ DATE } & \multirow{2}{*}{\multicolumn{2}{|c|}{$\begin{array}{l}\text { SUS. } \\
\text { SEO. } \\
\text { FACL } \\
\text { OTAM. } \\
\text { FINER } \\
\text { THAN }\end{array}$}} & \multicolumn{2}{|c|}{$\begin{array}{l}\text { SUS. } \\
\text { SEO. } \\
\text { FALL }\end{array}$} & \multicolumn{2}{|c|}{$\begin{array}{l}\text { SUS. } \\
\text { SEO. } \\
\text { FALL }\end{array}$} & \multicolumn{2}{|c|}{$\begin{array}{l}\text { SUS. } \\
\text { SEO. } \\
\text { FALC }\end{array}$} & \multicolumn{2}{|c|}{$\begin{array}{l}\text { SUS. } \\
\text { SEO. } \\
\text { FALC }\end{array}$} & \multicolumn{2}{|c|}{$\begin{array}{l}\text { SUS. } \\
\text { SED. } \\
\text { FAle }\end{array}$} & \multicolumn{2}{|c|}{$\begin{array}{l}\text { SUS. } \\
\text { SEO. } \\
\text { FALE }\end{array}$} & \multicolumn{2}{|c|}{$\begin{array}{l}\text { SUS. } \\
\text { SEO. } \\
\text { FALC }\end{array}$} \\
\hline & & & $\begin{array}{l}\text { DIAH } \\
\text { XIA } \\
\text { THI }\end{array}$ & ER & $\begin{array}{l}\text { OIAH } \\
\text { T FIN } \\
\text { THA }\end{array}$ & ER & $\begin{array}{l}\text { DIAA } \\
\text { X FIA } \\
\text { THA }\end{array}$ & IER & $\begin{array}{r}\text { OIAM } \\
\text { FIN } \\
\text { THA }\end{array}$ & INR & $\begin{array}{l}\text { THA } \\
\text { THA }\end{array}$ & & 8 FIN & IER & r fin & $\begin{array}{l}\text { NER } \\
\text { AN }\end{array}$ \\
\hline & .004 & MM & .008 & MM & .016 & MN & .031 & MM & .062 & MH & .125 & MH & .250 & MH & .500 & MH \\
\hline MAR. & & & & & & & & & & & & & & & & \\
\hline $19 \ldots$ & & - & & - & & - & & - & & - & & - & & - & & - \\
\hline $09 .$. & & 18 & & 22 & & 32 & & 47 & & 68 & & 87 & & 99 & & 100 \\
\hline $\begin{array}{l}\text { AUG. } \\
15 \ldots . .\end{array}$ & & - & & - & & - & & - & & - & & - & & - & & - \\
\hline
\end{tabular}




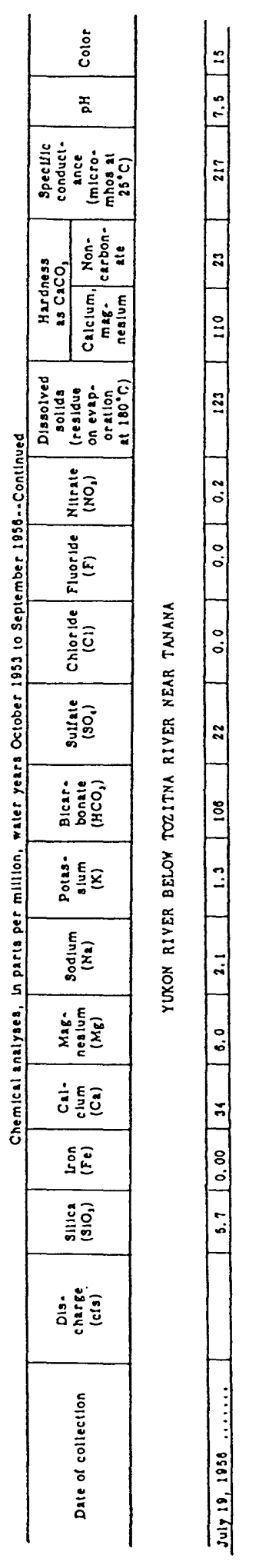

